\title{
Three-Component Coupling Involving Arynes, Aromatic Tertiary Amines and Aldehydes via Aryl-Aryl Amino Group Migration
}

\author{
Sachin Suresh Bhojgude, ${ }^{a}$ Dnyaneshwar R. Baviskar, ${ }^{a}$ Rajesh G. Gonnade ${ }^{b}$ and \\ Akkattu T. Biju*,a \\ ${ }^{a}$ Organic Chemistry Division, ${ }^{b}$ Center for Materials Characterization, \\ CSIR-National Chemical Laboratory (CSIR-NCL), \\ Dr. Homi Bhabha Road, Pune - 411008, India \\ at.biju@ncl.res.in
}

\section{Supporting Information}

1. General Information $\quad$ S2

2. General Procedure for the Optimization of the Reaction Conditions

3. Optimization Studies $\quad$ S3

4. General Procedure for the MCC Involving Arynes, Aldehydes and Tertiary Amines S4

5. General Procedure for the MCC involving Arynes, Isatins and Tertiary Amines $\quad$ S7

6. Mechanistic Experiments $\quad$ S8

7. Synthesis and Characterization of 2-Amino Benzhydrol Derivatives S11

10. ${ }^{1} \mathrm{H}$ and ${ }^{13} \mathrm{C}$ NMR Spectra of 2-Amino Benzhydrol Derivatives S43 


\section{General Information}

Unless otherwise specified, all reactions were carried out under an atmosphere of argon in flame-dried reaction vessels with Teflon screw caps. Reaction temperatures are reported as the temperature of the bath surrounding the reaction vessel. $30{ }^{\circ} \mathrm{C}$ corresponds to the room temperature of the lab, when the experiments were carried out. THF was freshly purified by distillation over Na-benzophenone and was transferred under argon. 18-Crown-6 was recrystallized from dry $\mathrm{CH}_{3} \mathrm{CN}$, and $\mathrm{KF}$ was dried by heating at $110{ }^{\circ} \mathrm{C}$ for $12 \mathrm{~h}$ and left to cool under argon and stored in glove box. The 2(trimethylsilyl)phenyl trifluoromethane sulfonate 1a and the other symmetric and unsymmetric aryne precursors $\mathbf{1 b}-\mathbf{1 h}$ were synthesized following literature procedure. ${ }^{1}$ The tertiary amines derivatives $3 \mathbf{a}-\mathbf{3 f}, \mathbf{3 h}, \mathbf{3 k}, \mathbf{3 n}$ and $\mathbf{3 o}$ were purchased from either from Sigma Aldrich or Alfa Aesar and used as received, without further purification. Tertiary amine derivatives $\mathbf{3 g}, \mathbf{3 i}, \mathbf{3} \mathbf{j}$, and $\mathbf{3 p}$ were synthesized following literature procedure. ${ }^{2}$

Analytical thin layer chromatography was performed on TLC Silica gel $60 \mathrm{~F}_{254}$. Visualization was accomplished with short wave UV light or $\mathrm{KMnO}_{4}$ staining solutions followed by heating. Chromatography was performed on silica gel (230-400 mesh) by standard techniques eluting with solvents as indicated.

All compounds were fully characterized. ${ }^{1} \mathrm{H}$ and ${ }^{13} \mathrm{C}$ NMR spectra were recorded on Bruker AV 400, 500 in solvents as indicated. Chemical shifts $(\delta)$ are given in ppm. The residual solvent signals were used as references and the chemical shifts converted to the TMS scale $\left(\mathrm{CDCl}_{3}: \delta \mathrm{H}=7.26 \mathrm{ppm}, \delta \mathrm{C}=77.16 \mathrm{ppm}\right)$. Infrared spectra were recorded on a Bruker Alpha-E Infrared Spectrophotometer. The wave numbers (n) of recorded IR-signals are quoted in $\mathrm{cm}^{-1}$. HRMS data were recorded on a Thermo Scientific Q-Exactive, Accela 1250 pump.

${ }^{1}$ (a) Sato, Y.; Tamura, T.; Kinbara, A.; Morib, M. Adv. Synth. Catal. 2007, 349, 647. (b) Peña, D.; Cobas, A.; Pérez, D.; Guitián, E. Synthesis 2002, 1454.

2 (a) Wagner, A.; Han, W.; Mayer, P.; Ofial, A. R. Adv. Synth. Catal. 2013, 355, 3058. (b) Zhang, L.; Peng, C.; Zhao, D.; Wang, Y.; Fu, H.-J.; Shen, Q.; Li, J.-X. Chem. Commun. 2012, 48, 5928. 


\section{General Procedure for the Optimization of the Reaction Conditions}

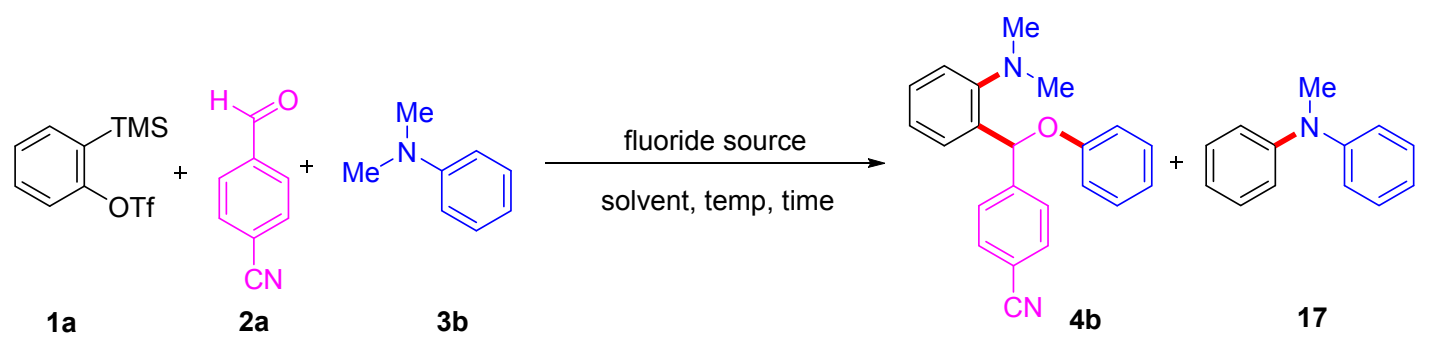

To a flame-dried screw-capped test tube equipped with a magnetic stir bar was added the fluoride source $(0.60 \mathrm{mmol})$, and aldehyde $2 \mathrm{a}(0.066 \mathrm{~g}, 0.50 \mathrm{mmol})$ inside the glove box. The mixture was dissolved in $2.0 \mathrm{~mL}$ of THF outside the glove box under argon and to this stirring solution was added $N, N$-dimethyl aniline $3 \mathbf{b}(0.032 \mathrm{~g}, 33 \mu \mathrm{L}, 0.25 \mathrm{mmol})$ and 2(trimethylsilyl)phenyl trifluoromethanesulfonate 1a $(0.090,73 \mu \mathrm{L}, 0.30 \mathrm{mmol}$ ) at room temperature. Then the screw-capped tube was stirred for the indicated time. The reaction mixture was then diluted with $\mathrm{CH}_{2} \mathrm{Cl}_{2}(2.0 \mathrm{~mL})$ and filtered through a short pad of silica gel and eluted with $\mathrm{CH}_{2} \mathrm{Cl}_{2}(10.0 \mathrm{~mL})$. The solvent was evaporated to obtain the crude product whose yield was determined by ${ }^{1} \mathrm{H}$ NMR analysis using $\mathrm{CH}_{2} \mathrm{Br}_{2}(18.0 \mu \mathrm{L}, 0.25 \mathrm{mmol})$ as the internal standard.

\section{Optimization Studies}

The present study was initiated by treating $N, N$-dimethyl aniline $\mathbf{3 b}$ and aldehyde $\mathbf{2 a}$ with the aryne generated in situ from 2-(trimethylsilyl)-aryl triflate 1a using 2.4 equiv each KF and 18-crown-6 at $60{ }^{\circ} \mathrm{C}$. A facile reaction took place resulting in the formation of 2-amino benzhydrol 4b in 73\% yield along with $N$-arylated product $N$-methyl- $N$-phenylaniline 17 in $20 \%$ yield (based on ${ }^{1} \mathrm{H}-\mathrm{NMR}$ spectroscopy, Table 1, entry 1). Reducing the reaction temperature to $40{ }^{\circ} \mathrm{C}$ improved the yield of $\mathbf{4 b}$ and reduced the formation of $\mathbf{1 7}$ (entry 2), further decrease in the reaction temperature to $30{ }^{\circ} \mathrm{C}$ did not increase the yield of $\mathbf{4 b}$ but $N$-arylated product 17 was formed in $<5 \%$ yield (entry 3). This indicates that as temperature decreases, yield of the MCC product $\mathbf{4 b}$ increases and the decrease in the formation of $N$-arylated product 17 . When the reaction was performed at $-10^{\circ} \mathrm{C}$ and slowly warmed to $\mathrm{rt}, \mathbf{4 b}$ was formed in $81 \%$ yield with traces of 17 (entry 4). Other fluoride sources, such as CsF, furnished less 76\% yield of $\mathbf{4 b}$ (entry 
5). Variation in amount of either 1a, 2a and $\mathbf{3 b}$ were not proved to be beneficial leading to decrease in the yield of MCC product $\mathbf{4 b}$ and increase in the formation of undesired $\mathrm{N}$-arylated product 17 (entries 6-9).<smiles>CS(=O)(=O)c1ccccc1[Te]</smiles><smiles>Cc1ccc(C=O)cc1</smiles>

2a<smiles>CN(C)c1ccccc1</smiles>

$3 b$

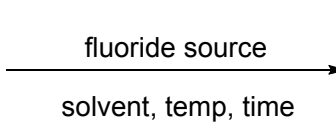

solvent, temp, time

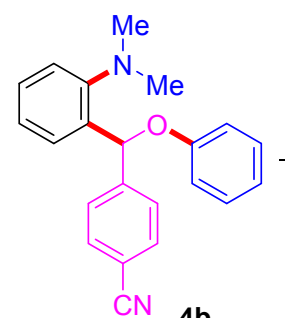

4b<smiles>CN(c1ccccc1)c1ccccc1</smiles>

17

\begin{tabular}{|c|c|c|c|l|l|l|c|c|}
\hline entry & $\begin{array}{c}\mathbf{1 a} \\
\text { (equiv) }\end{array}$ & $\begin{array}{c}\mathbf{2 a} \\
\text { (equiv) }\end{array}$ & $\begin{array}{c}\mathbf{3 b} \\
(\text { equiv) }\end{array}$ & $\mathrm{F}^{-}$source & solvent & $\begin{array}{c}\text { temp } \\
\left({ }^{\circ} \mathrm{C}\right)\end{array}$ & $\begin{array}{c}\text { yield of } \\
\mathbf{4 b}(\%)^{\mathrm{a}}\end{array}$ & $\begin{array}{c}\text { yield of } \\
\mathbf{1 7}(\%)^{\mathrm{a}}\end{array}$ \\
\hline 1 & 1.2 & 2 & 1 & $\mathrm{KF} / 18$-crown-6 & $\mathrm{THF}$ & 60 & 73 & 20 \\
\hline 2 & 1.2 & 2 & 1 & $\mathrm{KF} / 18$-crown-6 & $\mathrm{THF}$ & 40 & 83 & 10 \\
\hline 3 & 1.2 & 2 & 1 & $\mathrm{KF} / 18$-crown-6 & $\mathrm{THF}$ & 30 & 81 & $<5$ \\
\hline 4 & 1.2 & 2 & 1 & $\mathrm{KF} / 18$-crown-6 & $\mathrm{THF}$ & -10 to rt & $81(76)$ & $<5$ \\
\hline 5 & 1.2 & 2 & 1 & $\mathrm{CsF}$ & $\mathrm{CH}_{3} \mathrm{CN}$ & 30 & 76 & $<5$ \\
\hline 6 & 1.2 & 1.5 & 1 & $\mathrm{KF} / 18$-crown-6 & $\mathrm{THF}$ & 30 & 67 & $<5$ \\
\hline 7 & 1.5 & 1 & 1 & $\mathrm{KF} / 18$-crown-6 & $\mathrm{THF}$ & 30 & 35 & 21 \\
\hline 8 & 1.5 & 1.2 & 1 & $\mathrm{KF} / 18$-crown-6 & $\mathrm{THF}$ & 30 & 42 & 20 \\
\hline 9 & 1.5 & 1 & 1.2 & $\mathrm{KF} / 18$-crown-6 & $\mathrm{THF}$ & 30 & 49 & 12 \\
\hline
\end{tabular}

Standard conditions: $1 \mathbf{a}(0.30 \mathrm{mmol}), \mathbf{2 a}(0.50 \mathrm{mmol}), \mathbf{3 b}(0.25 \mathrm{mmol})$, fluoride source $(2.4$ equiv), solvent $(2.0 \mathrm{~mL})$, temperature and $12 \mathrm{~h}$. ${ }^{a}$ The yields were determined by ${ }^{1} \mathrm{H}$ NMR analysis of crude products using $\mathrm{CH}_{2} \mathrm{Br}_{2}$ as the internal standard. Isolated yield at $0.50 \mathrm{mmol}$ scale in parentheses.

\section{General Procedure for the MCC Involving Arynes, Aldehydes and Tertiary Amines}

\section{Procedure A}

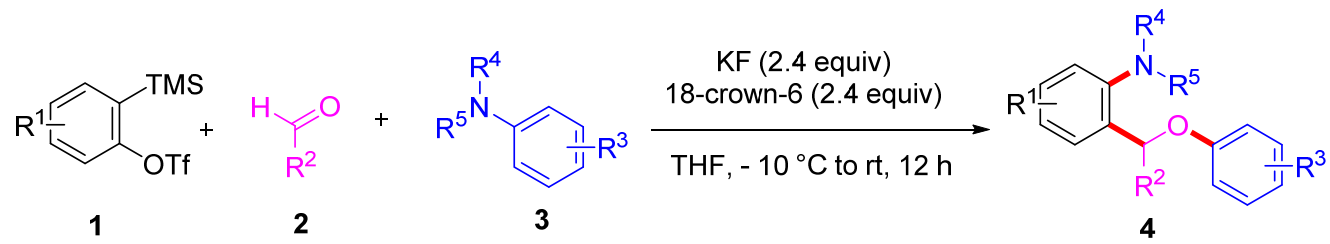

To a flame-dried screw-capped test tube equipped with a magnetic stir bar was added the 18-crown-6 $(0.317 \mathrm{~g}, 1.20 \mathrm{mmol}), \mathrm{KF}(0.070 \mathrm{~g}, 1.20 \mathrm{mmol})$ inside the glove box. The mixture 
was dissolved in $2.0 \mathrm{~mL}$ of THF outside the glove box under argon. To this mixture was added $1.0 \mathrm{mmol}$ of the aldehyde 2 (solid aldehydes were weighed in air and transferred to the screwcapped test tube by closing the argon flow and liquid aldehydes were transferred via syringe with argon flow), and tertiary amine $\mathbf{3}(0.50 \mathrm{mmol})$. The resultant reaction mixture was cooled to -10 ${ }^{\circ} \mathrm{C}$ and kept stirring for five minutes. To the stirring solution was added the aryne precursor $\mathbf{1}$ $(0.60 \mathrm{mmol})$. Then the reaction mixture was slowly warmed to $\mathrm{rt}$ and kept stirring for $12 \mathrm{~h}$. Then the reaction was quenched and the solvent was evaporated. Subsequently the crude residue was purified by flash column chromatography on silica gel to afford the corresponding 2-amino benzhydrol derivatives $\mathbf{4}$ in moderate to good yields.

\section{Procedure B}

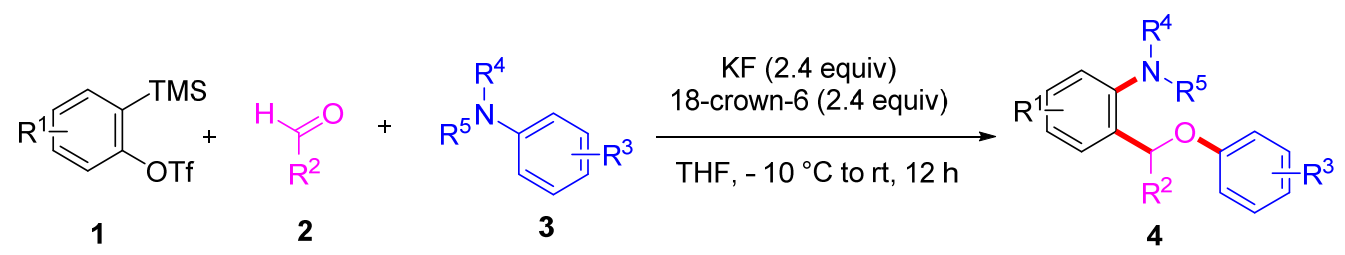

To a flame-dried screw-capped test tube equipped with a magnetic stir bar was added the 18-crown-6 $(0.317 \mathrm{~g}, 1.20 \mathrm{mmol}), \mathrm{KF}(0.070 \mathrm{~g}, 1.20 \mathrm{mmol})$ inside the glove box. The mixture was dissolved in $2.0 \mathrm{~mL}$ of THF outside the glove box under argon. To this mixture was added $1.0 \mathrm{mmol}$ of the aldehyde 2 (solid aldehydes were weighed in air and transferred to the screwcapped test tube by closing the argon flow and liquid aldehydes were transferred via syringe with argon flow), and tertiary amine $\mathbf{3}(0.50 \mathrm{mmol})$. The resultant reaction mixture was cooled to -10 ${ }^{\circ} \mathrm{C}$ and kept stirring for five minutes. To the stirring solution was added the aryne precursor $\mathbf{1}$ $(0.60 \mathrm{mmol})$. Then the reaction mixture was slowly warmed to $\mathrm{rt}$ and kept stirring for $12 \mathrm{~h}$. Then the reaction mixture was diluted with $\mathrm{CH}_{2} \mathrm{Cl}_{2}(4.0 \mathrm{~mL})$ and filtered through a short pad of silica gel and eluted with $\mathrm{CH}_{2} \mathrm{Cl}_{2}(20.0 \mathrm{~mL})$. The solvent was evaporated to obtain the crude reaction mixture, which was dissolved in $4.0 \mathrm{~mL}$ of $\mathrm{MeOH}$ and cooled to $0{ }^{\circ} \mathrm{C}$ followed by addition of $\mathrm{NaBH}_{4}(0.047 \mathrm{~g}, 1.25 \mathrm{mmol})$ in portions with stirring. The reaction mixture was slowly warmed to $\mathrm{rt}$, and stirred for $2 \mathrm{~h}$ at $\mathrm{rt}$. The reaction was quenched with $10 \mathrm{~mL}$ of water and the resulting aqueous solution extracted with $\mathrm{CH}_{2} \mathrm{Cl}_{2}(10 \mathrm{~mL} \times 3)$. The organic phase was combined, dried 
over anhydrous $\mathrm{Na}_{2} \mathrm{SO}_{4}$, filtered and concentrated to provide the crude product. The crude product was purified by flash column chromatography on silica gel to afford the corresponding 2-amino benzhydrol derivatives $\mathbf{4}$ in good yields.

In few cases, product of the MCC, 2-amino benzhydrol derivatives 4 have very close $R_{f}$ with excess aldehyde 3 used in the reaction, so attempted purification of the product by flash column chromatography on silica gel with different solvent systems was not successful. Excess aldehyde 3 reduced to corresponding benzyl alcohol which shows better $R_{f}$ difference with the product 4. This procedure allows easy purification of the product 4 by flash column chromatography.

\section{Procedure C}

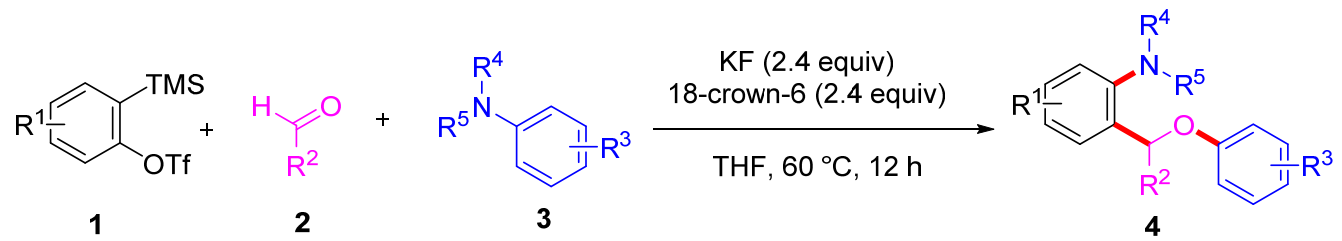

To a flame-dried screw-capped test tube equipped with a magnetic stir bar was added the 18-crown-6 (0.317 g, $1.2 \mathrm{mmol}), \mathrm{KF}(0.070 \mathrm{~g}, 1.2 \mathrm{mmol})$ and aldehyde $2(1.0 \mathrm{mmol})$ inside the glove box. The mixture was dissolved in $2.0 \mathrm{~mL}$ of THF outside the glove box under argon atmosphere and then to the stirring solution was added the tertiary amine $3(0.50 \mathrm{mmol})$ and the aryne precursor $1(0.60 \mathrm{mmol})$ at room temperature. Then the screw-capped tube was kept in a pre-heated oil bath at $60{ }^{\circ} \mathrm{C}$ for $12 \mathrm{~h}$. The reaction mixture was subsequently cooled. The solvent was evaporated and the crude residue was purified by flash column chromatography on silica gel to afford the corresponding 2-amino benzhydrol derivatives 4 in good yields. 


\section{General Procedure for the MCC involving Arynes, Isatins and Tertiary Amines}

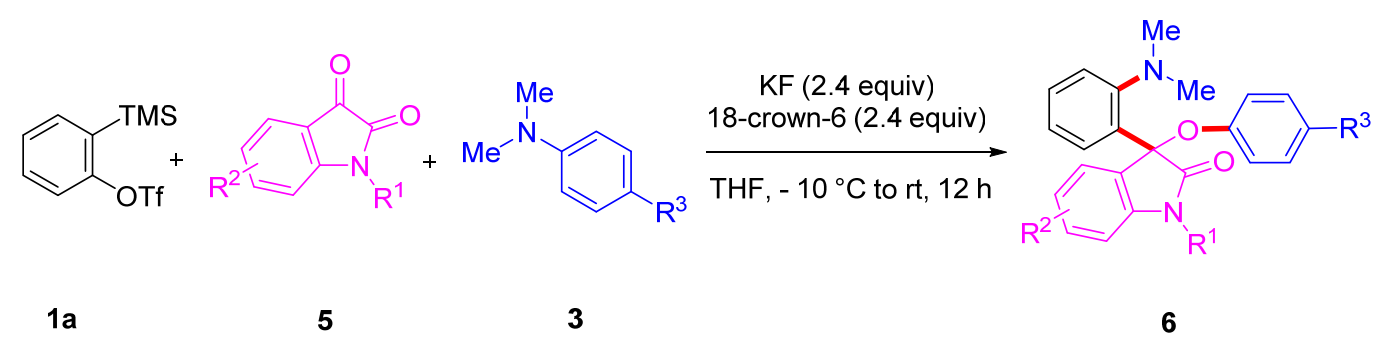

To a flame-dried screw-capped test tube equipped with a magnetic stir bar was added the 18-crown-6 (0.317 g, $1.2 \mathrm{mmol})$ and KF ( $0.070 \mathrm{~g}, 1.2 \mathrm{mmol})$ inside the glove box. Outside the glove box isatin $5(1.0 \mathrm{mmol})$ was added then the screw-capped tube was evacuated and backfilled with argon. The mixture was dissolved in THF $(2.0 \mathrm{~mL})$ under argon atmosphere. The resultant reaction mixture was cooled to $-10{ }^{\circ} \mathrm{C}$ and kept stirring for five minutes. To the stirring solution was added the tertiary amine $3(0.50 \mathrm{mmol})$ and the aryne precursor $1(0.60 \mathrm{mmol})$. Then the reaction mixture was slowly warmed to rt and kept stirring for $12 \mathrm{~h}$. Then the reaction quenched and the solvent was evaporated. Subsequently, the crude residue was purified by flash column chromatography on silica gel to afford the corresponding oxindole derivative $\mathbf{6}$ in good yields. 


\section{Mechanistic Experiments}

\section{a) Experiment to confirm the formation of tetrahedral intermediate (12) \\ Attempted MCC of aryne, aldehyde and N-methyl aniline}

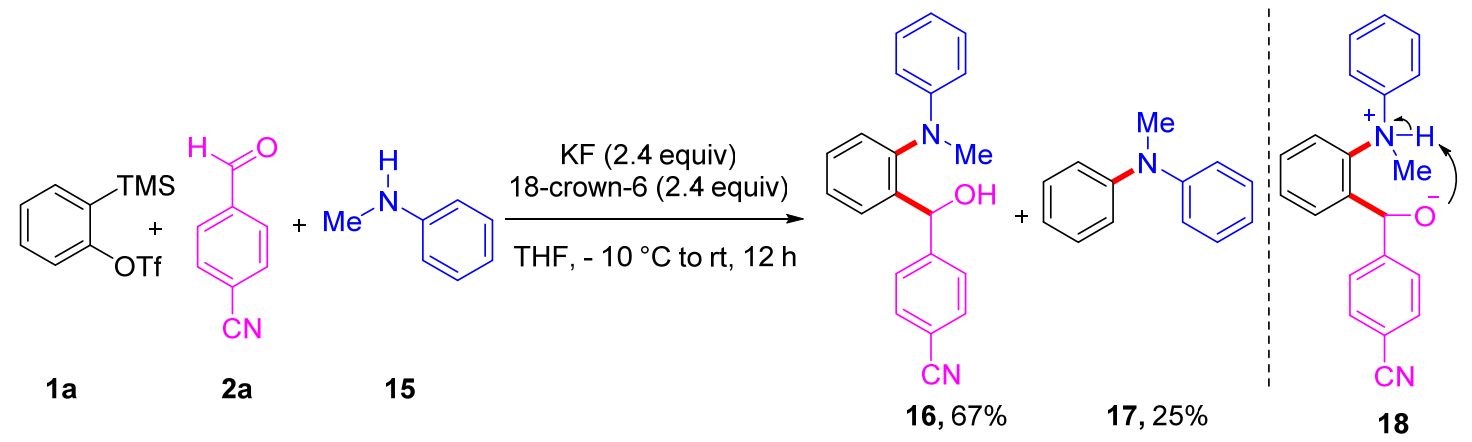

To a flame-dried screw-capped test tube equipped with a magnetic stir bar was added the 18-crown-6 (0.317 g, $1.2 \mathrm{mmol}), \mathrm{KF}(0.070 \mathrm{~g}, 1.2 \mathrm{mmol})$ and 4-formylbenzonitrile $2 \mathrm{a}(0.131 \mathrm{~g}$, $1.0 \mathrm{mmol}$ ) inside the glove box. The mixture was dissolved in $2.0 \mathrm{~mL}$ of THF outside the glove box under argon. The resultant reaction mixture was cooled to $-10{ }^{\circ} \mathrm{C}$ and kept stirring for five minutes. To the stirring solution was added $N$-methylaniline $15(0.053 \mathrm{~g}, 54 \mu \mathrm{L}, 0.50 \mathrm{mmol})$ and 2-(trimethylsilyl)phenyl trifluoromethanesulfonate $1 \mathrm{a}(0.179 \mathrm{~g}, 146 \mu \mathrm{L}, 0.60 \mathrm{mmol})$. Then the reaction mixture was slowly warmed to rt and kept stirring for $12 \mathrm{~h}$. Then the reaction quenched and the solvent was evaporated. The crude residue was purified by flash column chromatography on silica gel to afford the 4-(hydroxy(2-(methyl(phenyl)amino)phenyl)methyl)benzonitrile $\mathbf{1 6}$ in $67 \%$ yield with $N$-arylated product $N$-methyl- $N$-phenylaniline $17^{3}$ in $25 \%$ yield. In this case, the aryl-aryl amino group migration was not observed.

This result tends to indicates that the MCCs involving arynes, tertiary amines and aldehydes proceeds via the formation of tetrahedral intermediate 12 followed by aryl to aryl amino group migration (Scheme 5 of the manuscript). The tetrahedral intermediate 18 prefers intramolecular protonation which inhibits the nucleophilic aromatic substitution $\left(S_{N} A r\right)$ furnishing the product 16.

\footnotetext{
${ }^{3}$ Bhojgude, S. S.; Kaicharala, T.; Biju, A. T. Org. Lett. 2013, 15, 5452.
} 


\section{b) Experiments to check the possibility of intramolecular protonation of tetrahedral intermediate 12}

MCC of aryne, aldehyde and 2-phenyl-1,2,3,4-tetrahydroisoquinoline

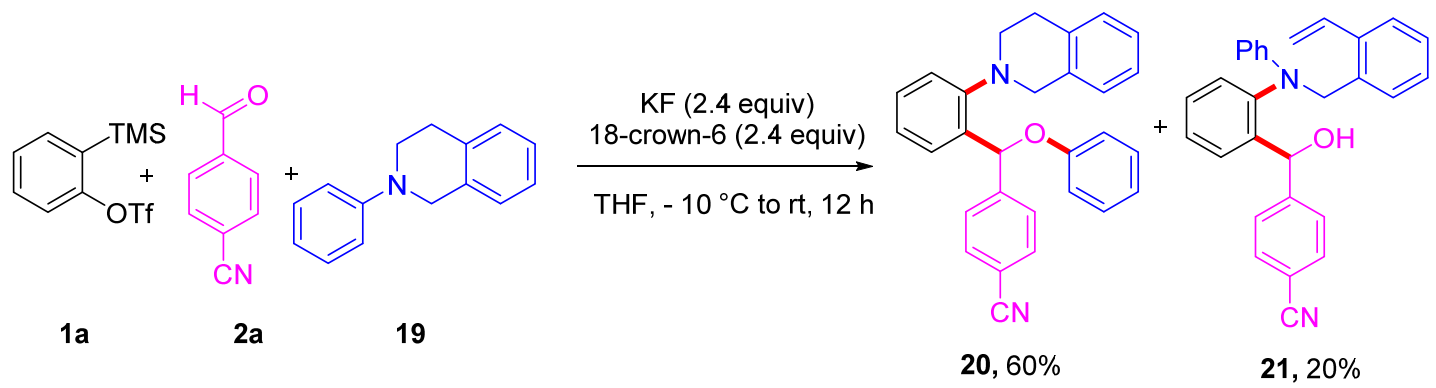

To a flame-dried screw-capped test tube equipped with a magnetic stir bar was added the 18-crown-6 (0.317 g, $1.2 \mathrm{mmol}), \mathrm{KF}(0.070 \mathrm{~g}, 1.2 \mathrm{mmol})$ and 4-formylbenzonitrile 2a (0.131 g, $1.0 \mathrm{mmol}$ ) inside the glove box. Outside the glove box 2-phenyl-1,2,3,4-tetrahydroisoquinoline $19(0.105 \mathrm{~g}, 0.50 \mathrm{mmol})$ was added then the screw-capped tube was evacuated and backfilled with argon. The mixture was dissolved in THF $(2.0 \mathrm{~mL})$ under argon atmosphere. The resultant reaction mixture was cooled to $-10{ }^{\circ} \mathrm{C}$ and kept stirring for five minutes. To the stirring solution 2-(trimethylsilyl)phenyl trifluoromethanesulfonate $\mathbf{1 a}(0.179 \mathrm{~g}, 146 \mu \mathrm{L}, 0.60 \mathrm{mmol})$ was added. Then the reaction mixture was slowly warmed to $\mathrm{rt}$ and kept stirring for $12 \mathrm{~h}$. Then the reaction quenched and the solvent was evaporated. The crude residue was purified by flash column chromatography on silica gel to afford the desired rearranged product $\mathbf{2 0}$ in $60 \%$ yield, and the styrene derivative 21 in $20 \%$ yield.

This experiment tends to indicate that the reaction offers $M C C$ product 20 proceeding via the formation of tetrahedral intermediate 12 followed by aryl to aryl amino group transfer along with the styrene derivative $\mathbf{2 1}$ which is formed by the intramolecular proton transfer of the initial amine-aryne-aldehyde adduct without the amino group migration. [eq 7 of manuscript]. 


\section{c) Cross-over Experiment: Experiments to confirm intramolecular aryl to aryl amino group transfer}

Reaction of aryne, aldehyde with a mixture of tertiary amines $3 a$ and $3 g$

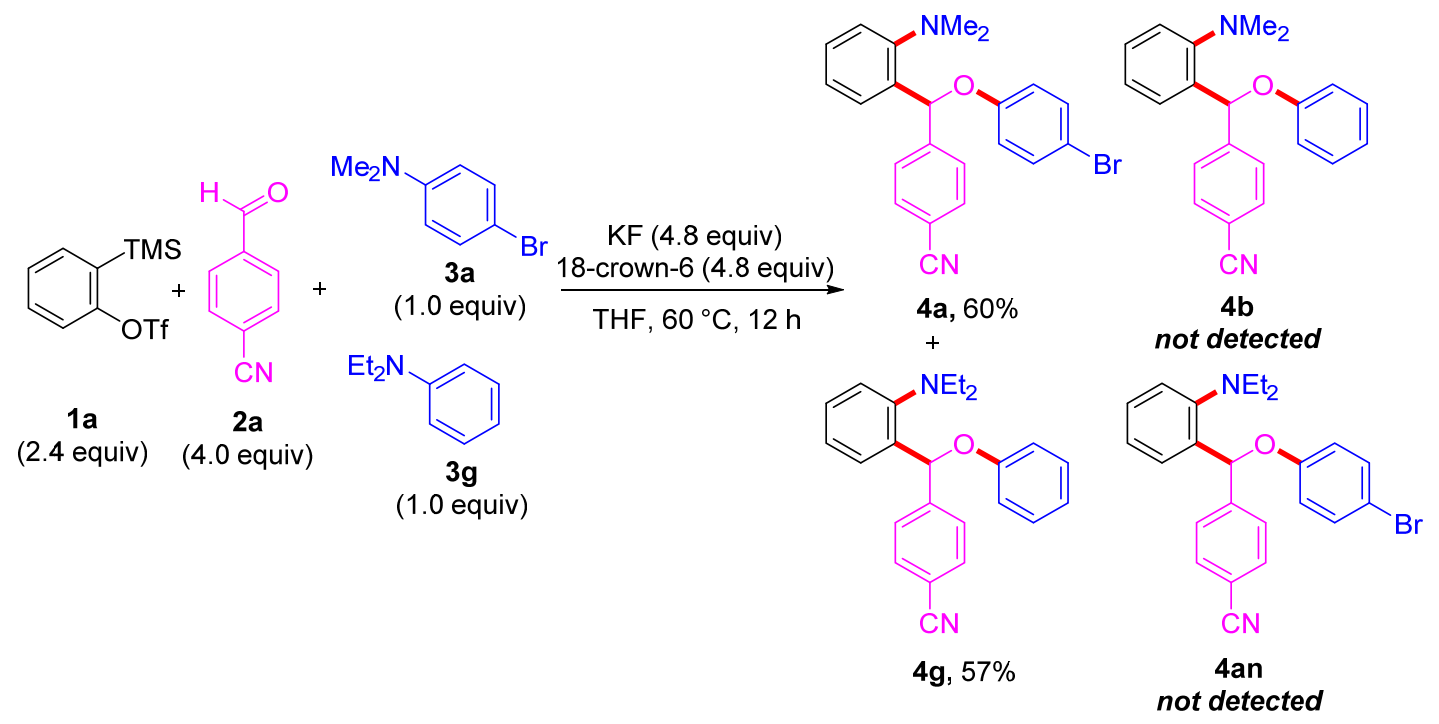

To a flame-dried screw-capped test tube equipped with a magnetic stir bar was added the 18-crown-6 (0.317 g, $1.2 \mathrm{mmol}), \mathrm{KF}(0.070 \mathrm{~g}, 1.2 \mathrm{mmol})$ and 4-formylbenzonitrile $2 \mathrm{a}(0.131 \mathrm{~g}$, $1.0 \mathrm{mmol}$ ) inside the glove box. The mixture was dissolved in $2.0 \mathrm{~mL}$ of THF outside the glove box under argon atmosphere and then to the stirring solution was added 4-bromo- $N, N$ dimethylaniline 3a (0.050 g, $0.25 \mathrm{mmol}), N, N$-diethylaniline $(0.037 \mathrm{~g}, 40 \mu \mathrm{L}, 0.25 \mathrm{mmol})$ and 2(trimethylsilyl)phenyl trifluoromethanesulfonate 1a $(0.179 \mathrm{~g}, 146 \mu \mathrm{L}, 0.60 \mathrm{mmol})$ at room temperature. Then the screw-capped tube was kept in a pre-heated oil bath at $60{ }^{\circ} \mathrm{C}$ for $12 \mathrm{~h}$. The reaction mixture was subsequently cooled. The solvent was evaporated and the crude residue was purified by flash column chromatography on silica gel afforded only two products 4-((4bromophenoxy)(2-(dimethylamino)phenyl)methyl)benzonitrile 4a (0.061 g, 60\% yield) and 4((2-(diethylamino)phenyl)(phenoxy)methyl)benzonitrile 4g (0.050 g, 57\% yield). Cross-over products $\mathbf{4 b}$ and $\mathbf{4 a n}$ were not detected.

This experiment demonstrates that the present multicomponent reaction proceeds via an intramolecular nucleophilic aromatic substitution $\left(S_{N} A r\right)$ of quaternary ammonium salt intermediate 12 leading to the aryl to aryl amino group transfer. [eq 8 of manuscript]. 


\section{Synthesis and Characterization of 2-Amino Benzhydrol Derivatives 4-((4-Bromophenoxy)(2-(dimethylamino)phenyl)methyl)benzonitrile (4a)}

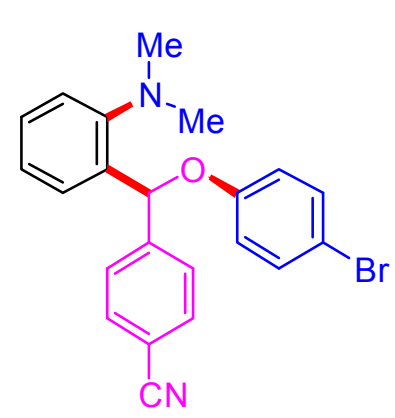

Following the general procedure A, treatment of 2-(trimethylsilyl) phenyltrifluoromethanesulfonate $1 \mathrm{a}(0.179 \mathrm{~g}, 146 \mu \mathrm{L}, 0.60 \mathrm{mmol})$ and 4-formylbenzonitrile 2a (0.131 g, $1.0 \mathrm{mmol})$ with 4-bromo- $N, N$ dimethylaniline $3 \mathrm{a}(0.100 \mathrm{~g}, 0.50 \mathrm{mmol})$ in the presence of $\mathrm{KF}(0.070$ $\mathrm{g}, 1.20 \mathrm{mmol})$ and 18-crown-6 $(0.317 \mathrm{~g}, 1.20 \mathrm{mmol})$ in THF $(2.0 \mathrm{~mL})$ at $-10{ }^{\circ} \mathrm{C}$ to $\mathrm{rt}$ for $12 \mathrm{~h}$ followed by flash column chromatography (Pet. ether/EtOAc $=98 / 02)$ of the crude reaction mixture using silica gel afforded 4-((4-bromo phenoxy)(2-(dimethylamino)phenyl)methyl)benzonitrile 4a as a colourless viscous oil ( $0.163 \mathrm{~g}$, $80 \%$ yield).

$\boldsymbol{R}_{\mathbf{f}}($ Pet. ether $/$ EtOAc $=95 / 05): 0.46 ;{ }^{1} \mathbf{H}$ NMR $\left(\mathbf{4 0 0} \mathbf{~ M H z}, \mathbf{C D C l}_{3}\right) \delta 7.64(\mathrm{~d}, J=8.2 \mathrm{~Hz}, 2 \mathrm{H})$, $7.54(\mathrm{~d}, J=8.2 \mathrm{~Hz}, 2 \mathrm{H}), 7.37-7.31(\mathrm{~m}, 4 \mathrm{H}), 7.28-7.26(\mathrm{~m}, 1 \mathrm{H}), 7.13(\mathrm{t}, J=7.4 \mathrm{~Hz}, 1 \mathrm{H}), 6.90(\mathrm{~s}$, 1H), $6.86(\mathrm{~d}, J=8.9 \mathrm{~Hz}, 2 \mathrm{H}), 2.67$ (s, 6H). ${ }^{13} \mathbf{C}$ NMR (100 MHz, $\left.\mathbf{C D C l}_{3}\right) \delta 156.98,152.70$, $146.67,134.75,132.37,132.31,129.54,128.67,128.14,124.97,120.98,118.79,117.65,113.50$, 111.50, 75.57, 45.91. HRMS (ESI) calculated $[\mathrm{M}+\mathrm{H}]^{+}$for $\mathrm{C}_{22} \mathrm{H}_{20} \mathrm{ON}_{2} \mathrm{Br}$ : 407.0754, found: 407.0750. FTIR $\left(\mathbf{c m}^{-1}\right)$ : 3020, 2941, 2787, 2230, 1591, 1489, 1227, 1006, 819, 760.

\section{4-((2-(Dimethylamino)phenyl)(phenoxy)methyl)benzonitrile (4b)}

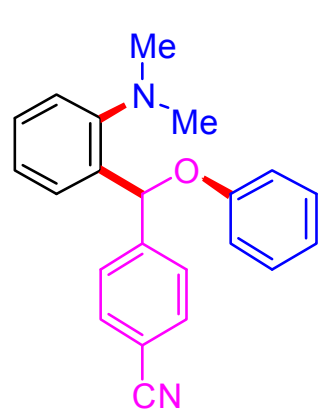

Following the general procedure A, treatment of 2-(trimethylsilyl)phenyl trifluoromethanesulfonate 1a $(0.179 \mathrm{~g}, 146 \mu \mathrm{L}, 0.60 \mathrm{mmol})$ and $4-$ formylbenzonitrile $2 \mathbf{a}(0.131 \mathrm{~g}, 1.0 \mathrm{mmol})$ with $N, N$-dimethylaniline $\mathbf{3 b}$ $(0.061 \mathrm{~g}, 64 \mu \mathrm{L}, 0.50 \mathrm{mmol})$ in the presence of $\mathrm{KF}(0.070 \mathrm{~g}, 1.20 \mathrm{mmol})$ and 18 -crown-6 $(0.317 \mathrm{~g}, 1.20 \mathrm{mmol})$ in THF $(2.0 \mathrm{~mL})$ at $-10{ }^{\circ} \mathrm{C}$ to $\mathrm{rt}$ for $12 \mathrm{~h}$ followed by flash column chromatography (Pet. ether $/$ EtOAc $=98 / 02$ )

of the crude reaction mixture using silica gel afforded 4-((2-(dimethylamino)phenyl)(phenoxy) methyl)benzonitrile $4 \mathbf{b}$ as a colourless viscous oil ( $0.125 \mathrm{~g}, 76 \%$ yield).

$\boldsymbol{R}_{\mathbf{f}}($ Pet. ether $/$ EtOAc $=95 / 05): 0.52 ;{ }^{1} \mathbf{H}$ NMR $\left(\mathbf{4 0 0} \mathbf{~ M H z}, \mathbf{C D C l}_{3}\right) \delta 7.55(\mathrm{~d}, J=8.0 \mathrm{~Hz}, 2 \mathrm{H})$, $7.50(\mathrm{~d}, J=8.0 \mathrm{~Hz}, 2 \mathrm{H}), 7.34$ (d, $J=7.7 \mathrm{~Hz}, 1 \mathrm{H}), 7.27-7.24(\mathrm{~m}, 1 \mathrm{H}), 7.21-7.15(\mathrm{~m}, 3 \mathrm{H}), 7.06(\mathrm{t}$, $J=7.3 \mathrm{~Hz}, 1 \mathrm{H}), 6.92-6.85(\mathrm{~m}, 4 \mathrm{H}), 2.61(\mathrm{~s}, 6 \mathrm{H}) .{ }^{13} \mathbf{C} \mathbf{~ N M R}\left(\mathbf{1 0 0} \mathbf{~ M H z}, \mathbf{C D C l}_{3}\right) \delta 157.89$, $152.59,147.28,135.37,132.25,129.54,129.32,128.72,128.07,124.90,121.26,120.93,118.90$, 
115.83, 111.23, 75.32, 45.86. HRMS (ESI) calculated $[\mathrm{M}+\mathrm{H}]^{+}$for $\mathrm{C}_{22} \mathrm{H}_{21} \mathrm{ON}_{2}: 329.1648$, found: 329.1644. FTIR (cm $\left.{ }^{-1}\right): 3021,2941,2833,2787,2231,1595,1492,1224,1022,760$.

\section{4-((2-(Dimethylamino)phenyl)(p-tolyloxy)methyl)benzonitrile (4c)}

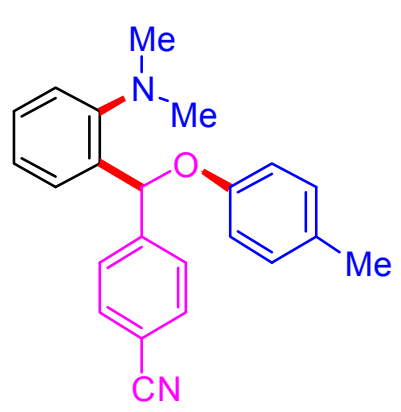

Following the general procedure $\mathbf{A}$, treatment of 2-(trimethylsilyl) phenyltrifluoromethanesulfonate $1 \mathrm{a}(0.179 \mathrm{~g}, 146 \mu \mathrm{L}, 0.60 \mathrm{mmol})$ and 4-formylbenzonitrile $\quad \mathbf{2 a}\left(\begin{array}{lllll}0.131 & \mathrm{~g}, & 1.0 & \mathrm{mmol}\end{array}\right)$ with $\quad N, N, 4-$ trimethylaniline $3 \mathbf{c}(0.068 \mathrm{~g}, 73 \mu \mathrm{L}, 0.50 \mathrm{mmol})$ in the presence of $\mathrm{KF}$ $(0.070 \mathrm{~g}, 1.20 \mathrm{mmol})$ and 18 -crown-6 $(0.317 \mathrm{~g}, 1.20 \mathrm{mmol})$ in THF $(2.0 \mathrm{~mL})$ at $-10{ }^{\circ} \mathrm{C}$ to $\mathrm{rt}$ for $12 \mathrm{~h}$ followed by flash column chromatography (Pet. ether/EtOAc $=98 / 02)$ of the crude reaction mixture using silica gel afforded 4-((2-(dimethylamino)phenyl)(p-tolyloxy)methyl)benzonitrile $\mathbf{4 c}$ as a colourless viscous oil ( $0.121 \mathrm{~g}, 70 \%$ yield $)$.

$\boldsymbol{R}_{\mathbf{f}}($ Pet. ether $/$ EtOAc $=95 / 05): 0.52 ;{ }^{1} \mathbf{H}$ NMR $\left(400 \mathbf{~ M H z}, \mathbf{C D C l}_{3}\right) \delta 7.62(\mathrm{~d}, J=8.3 \mathrm{~Hz}, 2 \mathrm{H})$, $7.56(\mathrm{~d}, J=8.3 \mathrm{~Hz}, 2 \mathrm{H}), 7.40\left(\mathrm{dd}, J_{I}=7.6 \mathrm{~Hz}, J_{2}=1.4 \mathrm{~Hz}, 1 \mathrm{H}\right), 7.34-7.30(\mathrm{~m}, 1 \mathrm{H}), 7.27-7.25$ (m, 1H), 7.14-7.10 (m, 1H), $7.04(\mathrm{~d}, J=8.5 \mathrm{~Hz}, 2 \mathrm{H}), 6.92(\mathrm{~s}, 1 \mathrm{H}), 6.87(\mathrm{~d}, J=8.6 \mathrm{~Hz}, 2 \mathrm{H}), 2.67$ (s, 6H), 2.27 (s, 3H). ${ }^{13} \mathbf{C}$ NMR (100 MHz, $\mathbf{C D C l}_{3}$ ) $\delta$ 155.80, 152.60, 147.51, 135.60, 132.24, 130.51, 130.01, 129.26, 128.75, 128.04, 124.89, 120.91, 118.95, 115.69, 111.16, 75.45, 45.86, 20.55. HRMS (ESI) calculated $[\mathrm{M}+\mathrm{H}]^{+}$for $\mathrm{C}_{23} \mathrm{H}_{23} \mathrm{ON}_{2}: 343.1805$, found: 343.1805 . FTIR $\left(\mathbf{c m}^{-}\right.$ 1): 3020, 2938, 2867, 2833, 2787, 2230, 1603, 1503, 1293, 1224, 1015, 762.

\section{Ethyl-4-((4-cyanophenyl)(2-(dimethylamino)phenyl)methoxy)benzoate (4d)}

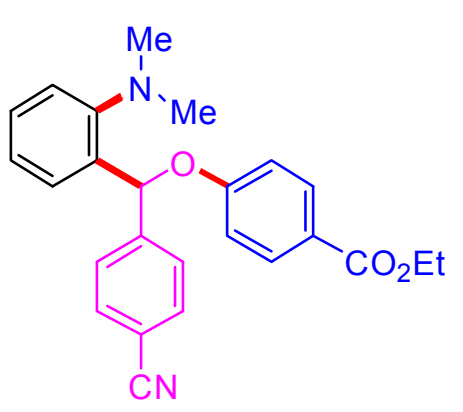

Following the general procedure A, treatment of 2-(trimethylsilyl) phenyltrifluoromethanesulfonate 1a $(0.179 \mathrm{~g}, 146 \mu \mathrm{L}, 0.60 \mathrm{mmol})$ and 4-formylbenzonitrile 2a $(0.131 \mathrm{~g}, 1.0 \mathrm{mmol})$ with ethyl 4(dimethylamino)benzoate $3 \mathbf{d}(0.097 \mathrm{~g}, 0.50 \mathrm{mmol})$ in the presence of $\mathrm{KF}(0.070 \mathrm{~g}, 1.20 \mathrm{mmol})$ and 18-crown-6 $(0.317 \mathrm{~g}, 1.20 \mathrm{mmol})$ in THF $(2.0 \mathrm{~mL})$ at $-10{ }^{\circ} \mathrm{C}$ to $\mathrm{rt}$ for $12 \mathrm{~h}$ followed by flash column chromatography of the crude reaction mixture using silica gel afforded ethyl 4(methyl(phenyl)amino)benzoate ${ }^{3}$ as a colourless oil (Pet. ether/EtOAc $=98 / 02 ; 0.030 \mathrm{~g}, 23 \%$ ) 
and ethyl-4-((4-cyanophenyl)(2-(dimethylamino)phenyl)methoxy)benzoate $\mathbf{4 d}$ as a colourless viscous oil ((Pet. ether/EtOAc $=96 / 04 ; 0.126$ g, 63\% yield).

$\boldsymbol{R}_{\mathbf{f}}($ Pet. ether $/$ EtOAc $=80 / 20): 0.61 ;{ }^{1} \mathbf{H}$ NMR $\left(400 \mathbf{~ M H z}, \mathbf{C D C l}_{3}\right) \delta 7.95(\mathrm{~d}, J=8.9 \mathrm{~Hz}, 2 \mathrm{H})$, $7.65(\mathrm{~d}, J=8.3 \mathrm{~Hz}, 2 \mathrm{H}), 7.56(\mathrm{~d}, J=8.3 \mathrm{~Hz}, 2 \mathrm{H}), 7.39-7.31(\mathrm{~m}, 2 \mathrm{H}), 7.28-7.26(\mathrm{~m}, 1 \mathrm{H}), 7.14-$ $7.10(\mathrm{~m}, 1 \mathrm{H}), 7.02-6.99(\mathrm{~m}, 3 \mathrm{H}), 4.34$ (q, $J=7.1 \mathrm{~Hz}, 2 \mathrm{H}), 2.69$ (s, 6H), 1.37 (t, $J=7.1 \mathrm{~Hz}, 3 \mathrm{H})$. ${ }^{13}$ C NMR (100 MHz, CDCl $) \delta$ 166.30, 161.50, 152.73, 146.41, 134.48, 132.33, 131.59, 129.61, $128.65,128.21,124.98,123.45,120.98,118.74,115.35,111.58,75.42,60.72,45.91,14.43$. HRMS (ESI) calculated $[\mathrm{M}+\mathrm{H}]^{+}$for $\mathrm{C}_{25} \mathrm{H}_{25} \mathrm{O}_{3} \mathrm{~N}_{2}: 401.1860$, found: 401.1852. FTIR (cm ${ }^{-1}$ ): 3019, 2932, 2861, 2787, 2230, 1709, 1601, 1500, 1455, 1364, 1279, 1237, 1171, 1109, 1011, 853,760 .

\section{4-((2-(Dimethylamino)phenyl)(m-tolyloxy)methyl)benzonitrile (4e)}

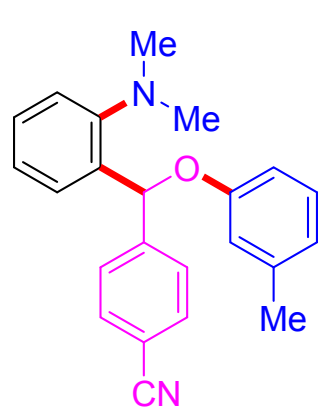

Following the general procedure $\mathbf{A}$, treatment of 2-(trimethylsilyl) phenyltrifluoromethanesulfonate 1a $(0.179 \mathrm{~g}, 146 \mu \mathrm{L}, 0.60 \mathrm{mmol})$ and $4-$ formylbenzonitrile $2 \mathbf{a}(0.131 \mathrm{~g}, 1.0 \mathrm{mmol})$ with $N, N, 3$-trimethylaniline $3 \mathbf{e}$ $(0.068 \mathrm{~g}, 72 \mu \mathrm{L}, 0.50 \mathrm{mmol})$ in the presence of $\mathrm{KF}(0.070 \mathrm{~g}, 1.20 \mathrm{mmol})$ and 18 -crown-6 $(0.317 \mathrm{~g}, 1.20 \mathrm{mmol})$ in THF $(2.0 \mathrm{~mL})$ at $-10{ }^{\circ} \mathrm{C}$ to $\mathrm{rt}$ for $12 \mathrm{~h}$ followed by flash column chromatography $($ Pet. ether/EtOAc $=98 / 02)$

of the crude reaction mixture using silica gel afforded 4-((2-(dimethylamino)phenyl) $(\mathrm{m}$ tolyloxy)methyl)benzonitrile $4 \mathbf{e}$ as a colourless viscous oil ( $0.126 \mathrm{~g}, 73 \%$ yield).

$\boldsymbol{R}_{\mathbf{f}}\left(\right.$ Pet. ether $/$ EtOAc $=$ 95/05): 0.52; ${ }^{1} \mathbf{H}$ NMR $\left(\mathbf{4 0 0} \mathbf{~ M H z}, \mathbf{C D C l}_{3}\right) \delta 7.64(\mathrm{~d}, J=8.4 \mathrm{~Hz}, 2 \mathrm{H})$, $7.59(\mathrm{~d}, J=8.4 \mathrm{~Hz}, 2 \mathrm{H}), 7.43\left(\mathrm{dd}, J_{I}=7.7 \mathrm{~Hz}, J_{2}=1.4 \mathrm{~Hz}, 1 \mathrm{H}\right), 7.36-7.32(\mathrm{~m}, 1 \mathrm{H}), 7.29\left(\mathrm{dd}, J_{I}\right.$ $\left.=8.0 \mathrm{~Hz}, J_{2}=1.2 \mathrm{~Hz}, 1 \mathrm{H}\right), 7.16-7.12(\mathrm{~m}, 2 \mathrm{H}), 6.99(\mathrm{~s}, 1 \mathrm{H}), 6.87(\mathrm{~s}, 1 \mathrm{H}), 6.81-6.78(\mathrm{~m}, 2 \mathrm{H}), 2.72$ (s, 6H), 2.32 (s, 3H). ${ }^{13} \mathbf{C}$ NMR (100 MHz, $\left.\mathbf{C D C l}_{3}\right) \delta$ 157.87, 152.55, 147.36, 139.48, 135.39, 132.18, 129.22, 128.72, 128.06, 124.85, 122.04, 120.81, 118.88, 116.60, 112.56, 111.14, 75.04, 45.83, 21.57. HRMS (ESI) calculated $[\mathrm{M}+\mathrm{H}]^{+}$for $\mathrm{C}_{23} \mathrm{H}_{23} \mathrm{ON}_{2}: 343.1805$, found: 343.1806 . FTIR $\left(\mathbf{c m}^{-1}\right)$ : 3020, 2940, 2231, 1597, 1491, 1255, 1217, 1158, 1033,762. 


\section{4-((3-Bromophenoxy)(2-(dimethylamino)phenyl)methyl) (4f)}

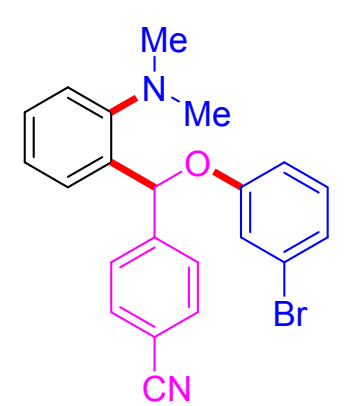

Following the general procedure A, treatment of 2-(trimethylsilyl)phenyl trifluoromethanesulfonate 1a $(0.179 \mathrm{~g}, 146 \mu \mathrm{L}, 0.60 \mathrm{mmol})$ and $4-$ formylbenzonitrile $\quad \mathbf{2 a} \quad(0.131 \mathrm{~g}, \quad 1.0 \mathrm{mmol})$ with 3-bromo- $N, N$ dimethylaniline $3 \mathrm{f}(0.100 \mathrm{~g}, 71 \mu \mathrm{L}, 0.50 \mathrm{mmol})$ in the presence of $\mathrm{KF}$ $(0.070 \mathrm{~g}, 1.20 \mathrm{mmol})$ and 18 -crown-6 $(0.317 \mathrm{~g}, 1.20 \mathrm{mmol})$ in THF $(2.0$ $\mathrm{mL}$ ) at $-10{ }^{\circ} \mathrm{C}$ to $\mathrm{rt}$ for $12 \mathrm{~h}$ followed by flash column chromatography (Pet. ether $/$ EtOAc $=98 / 02)$ of the crude reaction mixture using silica gel afforded 4-((3bromophenoxy)(2-(dimethylamino)phenyl)methyl) $\mathbf{4 f}$ as a yellow viscous oil $(0.162 \mathrm{~g}, 80 \%$ yield).

$\boldsymbol{R}_{\mathbf{f}}($ Pet. ether $/$ EtOAc $=95 / 05): 0.50 ;{ }^{1} \mathbf{H}$ NMR $\left(\mathbf{4 0 0} \mathbf{~ M H z}, \mathbf{C D C l}_{3}\right) \delta 7.64(\mathrm{~d}, J=8.3 \mathrm{~Hz}, 2 \mathrm{H})$, $7.53(\mathrm{~d}, J=8.3 \mathrm{~Hz}, 2 \mathrm{H}), 7.35-7.31(\mathrm{~m}, 2 \mathrm{H}), 7.27-7.25(\mathrm{~m}, 1 \mathrm{H}), 7.20(\mathrm{t}, J=1.9 \mathrm{~Hz}, 1 \mathrm{H}), 7.14-$ 7.03 (m, 3H), 6.90-6.87 (m, 2H) 2.69 (s, 6H). ${ }^{13} \mathbf{C}$ NMR (100 MHz, CDCl $) \delta 158.63,152.83$, 146.54, 134.43, 132.35, 130.65, 129.64, 129.36, 128.80, 128.32, 125.01, 124.34, 122.88, 120.90, 119.00, 118.83, 114.82, 111.60, 75.26, 46.02. HRMS (ESI) calculated $[\mathrm{M}+\mathrm{H}]^{+}$for $\mathrm{C}_{22} \mathrm{H}_{20} \mathrm{O}_{2} \mathrm{BrN2O}: 407.0754$, found: 407.0750. FTIR $\left(\mathbf{c m}^{-1}\right): 3020,2940,2788,2231,1585$, 1482, 1222, 1008, 765, 673.

\section{4-((2-(Diethylamino)phenyl)(phenoxy)methyl)benzonitrile (4g)}

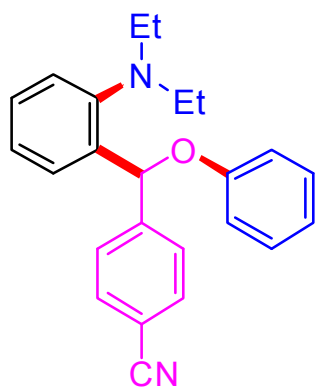

Following the general procedure $\mathbf{C}$, treatment of 2-(trimethylsilyl) phenyltrifluoromethanesulfonate $1 \mathrm{a}(0.179 \mathrm{~g}, 146 \mu \mathrm{L}, 0.60 \mathrm{mmol})$ and $4-$ formylbenzonitrile $2 \mathbf{a}(0.131 \mathrm{~g}, 1.0 \mathrm{mmol})$ with $N, N$-diethylaniline $\mathbf{3 g}$ $(0.075 \mathrm{~g}, 80 \mu \mathrm{L}, 0.50 \mathrm{mmol})$ in the presence of $\mathrm{KF}(0.070 \mathrm{~g}, 1.20 \mathrm{mmol})$ and 18-crown-6 $(0.317 \mathrm{~g}, 1.20 \mathrm{mmol})$ in THF $(2.0 \mathrm{~mL})$ at $60{ }^{\circ} \mathrm{C}$ for $12 \mathrm{~h}$ followed by flash column chromatography (Pet.ether/EtOAc $=98 / 02)$ of the crude reaction mixture using silica gel afforded 4-((2-(diethylamino)phenyl)(phenoxy) methyl)benzonitrile $4 \mathrm{~g}$ as a colourless viscous oil ( $0.116 \mathrm{~g}, 65 \%$ yield).

$\boldsymbol{R}_{\mathbf{f}}\left(\right.$ Pet. ether $/$ EtOAc $=$ 95/05): 0.48; ${ }^{1} \mathbf{H}$ NMR $\left(\mathbf{4 0 0} \mathbf{~ M H z}, \mathbf{C D C l}_{3}\right) \delta 7.62(\mathrm{~d}, J=8.2 \mathrm{~Hz}, 2 \mathrm{H})$, 7.52 (d, $J=8.2 \mathrm{~Hz}, 2 \mathrm{H}), 7.40$ (d, $J=7.7 \mathrm{~Hz}, 1 \mathrm{H}), 7.32$ (t, $J=7.1 \mathrm{~Hz}, 1 \mathrm{H}), 7.24-7.21$ (m, 3H), $7.14(\mathrm{t}, J=7.5 \mathrm{~Hz}, 1 \mathrm{H}), 6.98(\mathrm{~s}, 1 \mathrm{H}), 6.94-6.91(\mathrm{~m}, 3 \mathrm{H}), 2.93(\mathrm{q}, J=7.1 \mathrm{~Hz}, 4 \mathrm{H}), 0.95(\mathrm{t}, J=$ $7.1 \mathrm{~Hz}, 6 \mathrm{H}) .{ }^{\mathbf{1 3}} \mathbf{C}$ NMR (100 MHz, $\left.\mathbf{C D C l}_{3}\right) \delta$ 158.07, 149.75, 147.33, 137.14, 132.28, 129.50, 
$128.95,128.89,128.28,124.97,123.49,121.24,118.94,115.92,111.29,75.68,48.57,12.65$. HRMS (ESI) calculated $[\mathrm{M}+\mathrm{H}]^{+}$for $\mathrm{C}_{24} \mathrm{H}_{25} \mathrm{ON}_{2}: 357.1961$, found: 357.1955. FTIR (cm $\left.{ }^{-1}\right)$ : 3021, 2972, 2929, 2230, 1595, 1491, 1226, 1021, 760.

\section{4-((2-(Dibutylamino)phenyl)(phenoxy)methyl)benzonitrile (4h)}

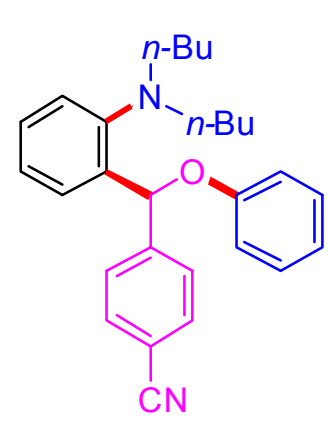

Following the general procedure $\mathbf{C}$, treatment of 2-(trimethylsilyl)phenyl trifluoromethanesulfonate 1a $(0.179 \mathrm{~g}, 146 \mu \mathrm{L}, 0.60 \mathrm{mmol})$ and $4-$ formylbenzonitrile $2 \mathbf{a}(0.131 \mathrm{~g}, 1.0 \mathrm{mmol})$ with $N, N$-dibutylaniline $\mathbf{3 h}$ $(0.103 \mathrm{~g}, 113 \mu \mathrm{L}, 0.50 \mathrm{mmol})$ in the presence of $\mathrm{KF}(0.070 \mathrm{~g}, 1.20 \mathrm{mmol})$ and [18]-crown-6 (0.317 g, $1.20 \mathrm{mmol})$ in THF $(2.0 \mathrm{~mL})$ at $60{ }^{\circ} \mathrm{C}$ for $12 \mathrm{~h}$ followed by flash column chromatography $($ Pet. ether $/$ EtOAc $=98 / 02)$ of the crude reaction mixture using silica gel afforded 4-((2-(dibutylamino)phenyl)(phenoxy) methyl)benzonitrile $4 \mathbf{h}$ as a yellow viscous oil $(0.118 \mathrm{~g}, 57 \%)$.

$\boldsymbol{R}_{\mathbf{f}}($ Pet. ether $/$ EtOAc $=97 / 03): 0.28 ;{ }^{1} \mathbf{H}$ NMR $\left(\mathbf{4 0 0} \mathbf{~ M H z}, \mathbf{C D C l}_{3}\right) \delta 7.64(\mathrm{~d}, J=8.2 \mathrm{~Hz}, 2 \mathrm{H})$, $7.52(\mathrm{~d}, J=8.0 \mathrm{~Hz}, 2 \mathrm{H}), 7.39-7.22(\mathrm{~m}, 5 \mathrm{H}), 7.15(\mathrm{t}, J=7.4 \mathrm{~Hz}, 1 \mathrm{H}), 7.00(\mathrm{~s}, 1 \mathrm{H}), 6.97-6.93$ (m, 3H), $2.88(\mathrm{t}, J=7.7 \mathrm{~Hz}, 4 \mathrm{H}), 1.45-1.32(\mathrm{~m}, 4 \mathrm{H}), 1.23-1.14(\mathrm{~m}, 4 \mathrm{H}), 0.83(\mathrm{t}, J=7.3 \mathrm{~Hz}$, 6H). ${ }^{13} \mathbf{C}$ NMR (100 MHz, $\left.\mathbf{C D C l}_{3}\right) \delta 158.10,150.81,147.29,136.56,132.25,129.45,129.19$, $128.96,128.32$, 124.72, 123.17, 121.22, 118.87, 115.87, 111.29, 75.61, 55.02, 29.84, 20.61, 14.04. HRMS (ESI) calculated $[\mathrm{M}+\mathrm{H}]^{+}$for $\mathrm{C}_{28} \mathrm{H}_{33} \mathrm{ON}_{2}: 413.2587$, found: 413.2587. FTIR (cm 1): 3021, 2958, 2867, 2821, 2231, 1595, 1490, 1417, 1221, 1021, 912, 768, 675.

\section{4-((2-(Butyl(methyl)amino)phenyl)(phenoxy)methyl)benzonitrile (4i)}

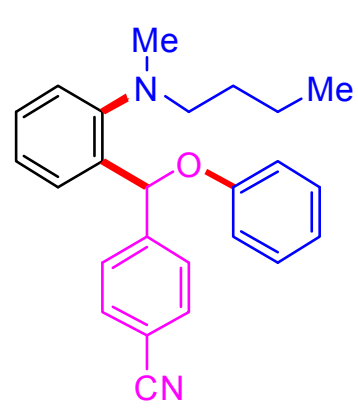

Following the general procedure $\mathbf{C}$, treatment of 2-(trimethylsilyl) phenyltrifluoromethanesulfonate $1 \mathrm{a}(0.179 \mathrm{~g}, 146 \mu \mathrm{L}, 0.60 \mathrm{mmol})$ and $4-$ formylbenzonitrile 2a $(0.131 \mathrm{~g}, 1.0 \mathrm{mmol})$ with $N$-butyl- $N$-methylaniline $3 \mathbf{i}(0.082 \mathrm{~g}, 0.50 \mathrm{mmol})$ in the presence of $\mathrm{KF}(0.070 \mathrm{~g}, 1.20 \mathrm{mmol})$ and 18-crown-6 $(0.317 \mathrm{~g}, 1.20 \mathrm{mmol})$ in THF $(2.0 \mathrm{~mL})$ at $60{ }^{\circ} \mathrm{C}$ for $12 \mathrm{~h}$ followed by flash column chromatography $($ Pet. ether/EtOAc $=98 / 02)$ of the crude reaction mixture using silica gel afforded 4-((2-(butyl(methyl)amino)phenyl) (phenoxy)methyl)benzonitrile $4 \mathbf{i}$ as a colourless viscous oil ( $0.132 \mathrm{~g}, 71 \%$ yield). 
$\boldsymbol{R}_{\mathbf{f}}($ Pet. ether $/$ EtOAc $=95 / 05): 0.65 ;{ }^{1} \mathbf{H}$ NMR (400 MHz, $\left.\mathbf{C D C l}_{3}\right) \delta 7.66(\mathrm{~d}, J=8.3 \mathrm{~Hz}, 2 \mathrm{H})$, $7.59(\mathrm{~d}, J=8.3 \mathrm{~Hz}, 2 \mathrm{H}), 7.46\left(\mathrm{dd}, J_{1}=7.7 \mathrm{~Hz}, J_{2}=1.3 \mathrm{~Hz}, 1 \mathrm{H}\right), 7.39-7.34(\mathrm{~m}, 1 \mathrm{H}), 7.31-7.26$ (m, 3H), 7.18 (t, $J=7.4 \mathrm{~Hz}, 1 \mathrm{H}), 7.03-6.96$ (m, 4H), 2.96-2.81 (m, 2H), 2.64 (s, 3H), 1.58-1.46 $(\mathrm{m}, 2 \mathrm{H}), 1.33-1.23(\mathrm{~m}, 2 \mathrm{H}), 0.89$ (t, $J=7.3 \mathrm{~Hz}, 3 \mathrm{H}) .{ }^{13} \mathbf{C}$ NMR (100 MHz, CDCl $\left.\mathbf{3}\right) \delta 157.93$, 152.32, 147.19, 135.89, 132.21, 129.45, 129.18, 128.67, 128.16, 124.88, 122.02, 121.19, 118.85, 115.80, 111.20, 75.45, 57.75, 43.33, 30.18, 20.43, 14.03. HRMS (ESI) calculated $[\mathrm{M}+\mathrm{H}]^{+}$for $\mathrm{C}_{25} \mathrm{H}_{27} \mathrm{ON}_{2}$ : 371.2118, found: 371.2117. FTIR (cm $\left.{ }^{-1}\right): 3020,2953,2863,2802,2230,1595$, $1491,1224,1020,759$.

\section{4-((2-(Benzyl(methyl)amino)phenyl)(phenoxy)methyl)benzonitrile (4j)}

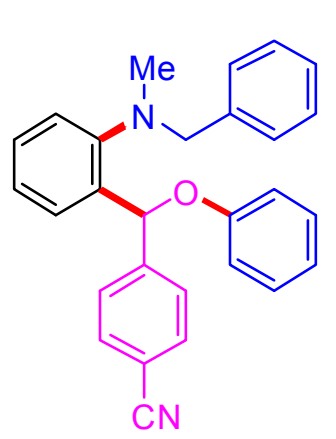

Following the general procedure $\mathbf{C}$, treatment of 2-(trimethylsilyl) phenyltrifluoromethanesulfonate $1 \mathrm{a}(0.179 \mathrm{~g}, 146 \mu \mathrm{L}, 0.60 \mathrm{mmol})$ and 4formylbenzonitrile $2 \mathrm{a}(0.131 \mathrm{~g}, 1.0 \mathrm{mmol})$ with $N$-benzyl- $N$-methylaniline 3j (0.099 g, $0.50 \mathrm{mmol})$ in the presence of $\mathrm{KF}(0.070 \mathrm{~g}, 1.20 \mathrm{mmol})$ and 18-crown-6 (0.317 g, $1.20 \mathrm{mmol})$ in $\operatorname{THF}(2.0 \mathrm{~mL})$ at $60{ }^{\circ} \mathrm{C}$ for $12 \mathrm{~h}$ followed by flash column chromatography (Pet. ether/EtOAc $=98 / 02)$ of the crude reaction mixture using silica gel afforded 4-((2-(benzyl(methyl)amino)phenyl) (phenoxy)methyl)benzonitrile $\mathbf{4 j}$ as a colourless viscous oil ( $0.084 \mathrm{~g}, 42 \%$ yield).

$\boldsymbol{R}_{\mathbf{f}}($ Pet. ether $/$ EtOAc $=95 / 05): 0.60 ;{ }^{1} \mathbf{H}$ NMR (400 MHz, $\left.\mathbf{C D C l}_{3}\right) \delta 7.63(\mathrm{~d}, J=8.2 \mathrm{~Hz}, 2 \mathrm{H})$, 7.49 (d, $J=8.2 \mathrm{~Hz}, 2 \mathrm{H}), 7.40-7.37$ (m, 3H), 7.32-7.29 (m, 5H), 7.24-7.17 (m, 3H), 7.03 (s, 1H), $6.94(\mathrm{t}, J=7.3 \mathrm{~Hz}, 1 \mathrm{H}), 6.88(\mathrm{~d}, J=8.0 \mathrm{~Hz}, 2 \mathrm{H}), 4.06$ (q, $J=13.5 \mathrm{~Hz}, 2 \mathrm{H}), 2.55(\mathrm{~s}, 3 \mathrm{H}) .{ }^{13} \mathrm{C}$ NMR (100 MHz, $\left.\mathbf{C D C l}_{3}\right) \delta$ 157.96, 151.89, 147.04, 138.25, 135.88, 132.34, 129.57, 129.34, 128.91, 128.81, 128.51, 128.26, 127.38, 125.43, 122.66, 121.30, 118.91, 115.87, 111.34, 75.69, 62.30, 43.36. HRMS (ESI) calculated $[\mathrm{M}+\mathrm{H}]^{+}$for $\mathrm{C}_{28} \mathrm{H}_{25} \mathrm{ON}_{2}$ : 405.1961, found: 405.1961. FTIR (cm $\left.{ }^{-1}\right):$ 3021, 2941, 2800, 2231, 1595, 1491, 1220, 1022, 763.

\section{4-((2-(Dimethylamino)phenyl)(naphthalen-1-yloxy)methyl)benzonitrile (4k)}

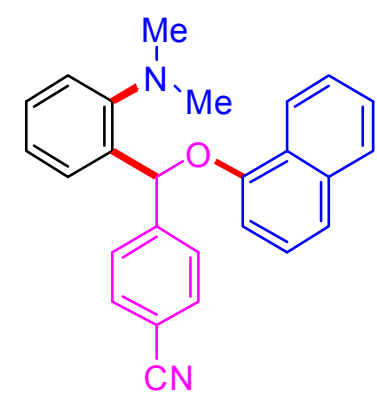

Following the general procedure A, treatment of 2-(trimethylsilyl) phenyltrifluoromethanesulfonate $1 \mathbf{a}(0.179 \mathrm{~g}, 146 \mu \mathrm{L}, 0.60 \mathrm{mmol})$ and 4-formylbenzonitrile $\quad \mathbf{2 a}(0.131 \mathrm{~g}, \quad 1.0 \mathrm{mmol})$ with $\mathrm{N}, \mathrm{N}$ dimethylnaphthalen-1-amine $3 \mathbf{k}(0.086 \mathrm{~g}, 0.50 \mathrm{mmol})$ in the presence of 
$\mathrm{KF}(0.070 \mathrm{~g}, 1.20 \mathrm{mmol})$ and 18 -crown-6 $(0.317 \mathrm{~g}, 1.20 \mathrm{mmol})$ in $\mathrm{THF}(2.0 \mathrm{~mL})$ at $-10{ }^{\circ} \mathrm{C}$ to $\mathrm{rt}$ for $12 \mathrm{~h}$ followed by flash column chromatography (Pet. ether/EtOAc $=98 / 02)$ of the crude reaction mixture using silica gel afforded 4-((2-(dimethylamino)phenyl)(naphthalen-1yloxy)methyl)benzonitrile $4 \mathbf{k}$ as a white solid $(0.133 \mathrm{~g}, 70 \%$ yield $)$.

$\boldsymbol{R}_{\mathbf{f}}($ Pet. ether $/$ EtOAc $=95 / 05): 0.44 ;{ }^{1} \mathbf{H}$ NMR $\left(\mathbf{4 0 0} \mathbf{~ M H z}, \mathbf{C D C l}_{3}\right) \delta 8.40-8.38(\mathrm{~m}, 1 \mathrm{H}), 7.84-$ $7.82(\mathrm{~m}, 1 \mathrm{H}), 7.66-7.62(\mathrm{~m}, 4 \mathrm{H}), 7.55-7.50(\mathrm{~m}, 3 \mathrm{H}), 7.44(\mathrm{~d}, J=8.2 \mathrm{~Hz}, 1 \mathrm{H}), 7.37-7.28(\mathrm{~m}, 3 \mathrm{H})$, 7.17-7.13 (m, 2H), $6.84(\mathrm{~d}, J=7.7 \mathrm{~Hz}, 1 \mathrm{H}), 2.71(\mathrm{~s}, 6 \mathrm{H}) .{ }^{13} \mathbf{C} \mathbf{~ N M R}\left(\mathbf{1 0 0} \mathbf{~ M H z}, \mathbf{C D C l}_{3}\right) \delta$ 153.39, 152.70, 147.34, 135.46, 134.71, 132.37, 129.42, 128.66, 128.05, 127.68, 126.58, 125.88, 125.49, 125.01, 122.24, 120.97, 120.82, 118.96, 111.36, 106.91, 75.53, 45.93. HRMS (ESI) calculated $[\mathrm{M}+\mathrm{H}]^{+}$for $\mathrm{C}_{26} \mathrm{H}_{23} \mathrm{ON}_{2}: 379.1805$, found: 379.1802. FTIR $\left(\mathbf{c m}^{-1}\right): 3059,3020,2937$, 2862, 2786, 2230, 1586, 1496, 1454, 1399, 1271, 1227, 1097, 1057, 759.

\section{(E)-4-((4-(2-(5-Bromothiophen-2-yl)vinyl)phenoxy)(2-(dimethylamino)phenyl)methyl)}

benzonitrile (4I)

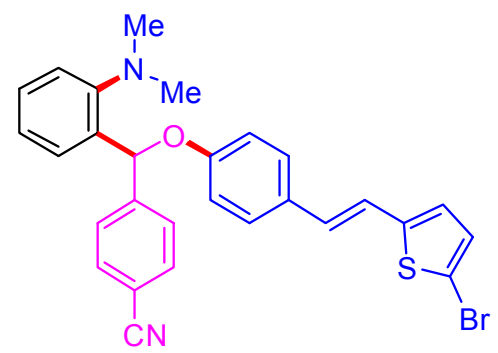

Following the general procedure A, treatment of 2(trimethylsilyl)phenyl trifluoromethanesulfonate 1a $(0.179 \mathrm{~g}, 146$ $\mu \mathrm{L}, 0.60 \mathrm{mmol})$ and 4-formylbenzonitrile $2 \mathrm{a}(0.131 \mathrm{~g}, 1.0 \mathrm{mmol})$ with (E)-4-(2-(5-bromothiophen-2-yl)vinyl)- $N, N$-dimethylaniline $31(0.154 \mathrm{~g}, 0.50 \mathrm{mmol})$ in the presence of $\mathrm{KF}(0.070 \mathrm{~g}, 1.20$ $\mathrm{mmol})$ and [18]-crown-6 (0.317 g, $1.20 \mathrm{mmol})$ in THF $(2.0 \mathrm{~mL})$ at $-10{ }^{\circ} \mathrm{C}$ to $\mathrm{rt}$ for $12 \mathrm{~h}$ followed by flash column chromatography (Pet. ether $\left./ \mathrm{EtOAc}=98 / 02\right)$ of the crude reaction mixture using silica gel afforded (E)-4-((4-(2-(5-bromothiophen-2yl)vinyl)phenoxy)(2-(dimethylamino)phenyl)methyl)benzonitrile $\mathbf{4 l}$ as a yellow viscous oil $(0.124 \mathrm{~g}, 48 \%)$.

$\boldsymbol{R}_{\mathbf{f}}($ Pet. ether $/$ EtOAc $=95 / 05): 0.33 ;{ }^{1} \mathbf{H}$ NMR $\left(\mathbf{4 0 0} \mathbf{~ M H z}, \mathbf{C D C l}_{3}\right) \delta 7.63($ d, $J=8.4 \mathrm{~Hz}, 2 \mathrm{H})$, $7.56(\mathrm{~d}, J=8.3 \mathrm{~Hz}, 2 \mathrm{H}), 7.38\left(\mathrm{dd}, J_{1}=7.8, J_{2}=1.3 \mathrm{~Hz}, 1 \mathrm{H}\right), 7.35-7.26(\mathrm{~m}, 4 \mathrm{H}), 7.15-7.11(\mathrm{~m}$, 1H), 6.96-6.93 (m, 5H), 6.75-6.71 (m, 2H), 2.68 (s, 6H). ${ }^{13} \mathbf{C}$ NMR (100 MHz, $\left.\mathbf{C D C l}_{3}\right) \delta$ 157.77, 152.66, 146.97, 144.86, 135.06, 132.30, 130.50, 129.90, 129.45, 128.73, 128.29, 128.12, 127.68, 125.71, 124.94, 120.95, 119.53, 118.87, 116.13, 111.38, 45.91. HRMS (ESI) calculated [M+H] ${ }^{+}$ for $\mathrm{C}_{28} \mathrm{H}_{24} \mathrm{BrOSN}_{2}$ : 515.0787, found: 515.0789. FTIR ( $\left.\mathbf{c m}^{-1}\right)$ : 3021, 2942, 2788, 2231, 1604, 1504, 1223, 1011, 766, 670 . 


\section{Ethyl (E)-3-(4-((4-cyanophenyl)(2-(dimethylamino)phenyl)methoxy)phenyl)acrylate (4m)}

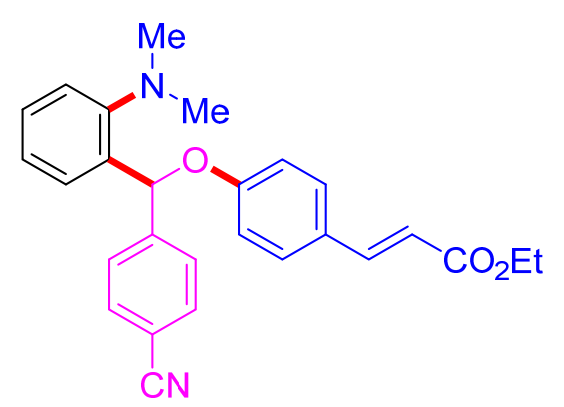

Following the general procedure $\mathbf{B}$ (reduction carried out in EtOH as a solvent), treatment of 2-(trimethylsilyl)phenyl trifluoromethanesulfonate 1a $(0.179 \mathrm{~g}, 146 \mu \mathrm{L}, 0.60 \mathrm{mmol})$ and 4-formylbenzonitrile $2 \mathrm{a}(0.131 \mathrm{~g}, 1.0 \mathrm{mmol})$ with ethyl (E)-3-(4-(dimethylamino)phenyl) acrylate $3 \mathbf{m}(0.110 \mathrm{~g}, 0.50$ $\mathrm{mmol})$ in the presence of $\mathrm{KF}(0.070 \mathrm{~g}, 1.20 \mathrm{mmol})$ and 18 crown-6 $(0.317 \mathrm{~g}, 1.20 \mathrm{mmol})$ in THF $(2.0 \mathrm{~mL})$ at $-10^{\circ} \mathrm{C}$ to $\mathrm{rt}$ for $12 \mathrm{~h}$ followed by flash column chromatography (Pet. ether/EtOAc $=94 / 06)$ of the crude reaction mixture using silica gel afforded ethyl (E)-3-(4-((4-cyanophenyl)(2-(dimethylamino)phenyl)methoxy)phenyl)acrylate $4 \mathbf{m}$ as a colourless viscous oil ( $0.154 \mathrm{~g}, 72 \%$ yield).

$\boldsymbol{R}_{\mathbf{f}}($ Pet. ether $/ \mathrm{EtOAc}=80 / 20): 0.52 ;{ }^{1} \mathbf{H}$ NMR $\left(\mathbf{4 0 0} \mathbf{~ M H z}, \mathbf{C D C l}_{3}\right) \delta$ 7.65-7.60 (m, 3H), $7.55(\mathrm{~d}$, $J=8.3 \mathrm{~Hz}, 2 \mathrm{H}), 7.43$ (d, $J=8.7 \mathrm{~Hz}, 2 \mathrm{H}), 7.40-7.32(\mathrm{~m}, 2 \mathrm{H}), 7.28$ (d, $J=7.1 \mathrm{~Hz}, 1 \mathrm{H}), 7.13$ (t, $J$ $=7.1 \mathrm{~Hz}, 1 \mathrm{H}), 6.99-6.96(\mathrm{~m}, 3 \mathrm{H}), 6.30(\mathrm{~d}, J=16.0 \mathrm{~Hz}, 1 \mathrm{H}), 4.26(\mathrm{q}, J=7.1 \mathrm{~Hz}, 2 \mathrm{H}), 2.69(\mathrm{~s}$, 6H), $1.34(\mathrm{t}, J=7.1 \mathrm{~Hz}, 3 \mathrm{H}) .{ }^{13} \mathbf{C}$ NMR (100 MHz, $\left.\mathbf{C D C l}_{3}\right) \delta 167.31,159.57,152.66,146.60$, 144.10, 134.68, 132.31, 129.73, 129.54, 128.66, 128.13, 127.69, 124.95, 120.97, 118.76, 116.08, 111.50, 75.43, 60.41, 45.89, 14.39. HRMS (ESI) calculated $[\mathrm{M}+\mathrm{H}]^{+}$for $\mathrm{C}_{27} \mathrm{H}_{27} \mathrm{O}_{3} \mathrm{~N}_{2}: 427.2016$, found: 427.2015. FTIR ( $\left.\mathbf{c m}^{-1}\right)$ : 3020, 2941, 2230, 1705, 1633, 1602, 1502, 1455, 1312, 1236 , $1170,1035,998,824,759$.

\section{Compound (4n)}

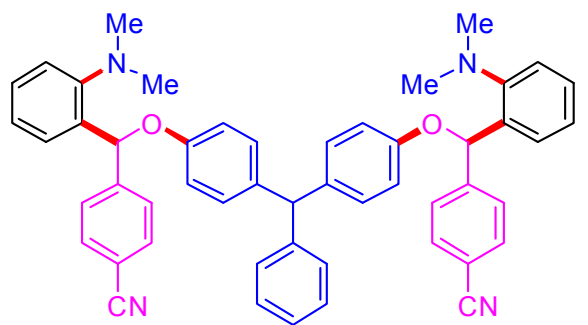

Following the general procedure $\mathbf{A}$, treatment of 2(trimethylsilyl)phenyl trifluoromethanesulfonate 1a $(0.179$ $\mathrm{g}, 146 \mu \mathrm{L}, 0.60 \mathrm{mmol})$ and 4-formylbenzonitrile 2a $(0.131$ g, $\quad 1.0$ mmol) with 4,4 '-(phenylmethylene)bis $(N, N$ dimethylaniline $) 3 \mathbf{n}(0.083 \mathrm{~g}, 0.25 \mathrm{mmol})$ in the presence of $\mathrm{KF}(0.070 \mathrm{~g}, 1.20 \mathrm{mmol})$ and [18]-crown-6 $(0.317 \mathrm{~g}, 1.20 \mathrm{mmol})$ in THF $(2.0 \mathrm{~mL})$ at $-10^{\circ} \mathrm{C}$ to $\mathrm{rt}$ for $12 \mathrm{~h}$ followed by flash column chromatography (Pet. ether $/ \mathrm{EtOAc}=90 / 10)$ of the crude reaction mixture using silica gel afforded compund $\mathbf{4 n}$ as a yellow solid $(0.094 \mathrm{~g}, 50 \%)$. 
$\boldsymbol{R}_{\mathbf{f}}($ Pet. ether $/$ EtOAc $=80 / 20): 0.42 ;{ }^{1} \mathbf{H}$ NMR $\left(400 \mathbf{~ M H z}, \mathbf{C D C l}_{3}\right) \delta 7.63(\mathrm{~d}, J=8.3 \mathrm{~Hz}, 4 \mathrm{H})$, $7.56(\mathrm{~d}, J=8.2 \mathrm{~Hz}, 4 \mathrm{H}), 7.43(\mathrm{~d}, J=7.6 \mathrm{~Hz}, 2 \mathrm{H}), 7.37-7.26(\mathrm{~m}, 6 \mathrm{H}), 7.21(\mathrm{~d}, J=7.1 \mathrm{~Hz}, 1 \mathrm{H})$, $7.14(\mathrm{t}, J=7.4 \mathrm{~Hz}, 2 \mathrm{H}), 7.08(\mathrm{~d}, J=7.2 \mathrm{~Hz}, 2 \mathrm{H}), 6.96(\mathrm{~d}, J=8.5 \mathrm{~Hz}, 4 \mathrm{H}), \quad 6.90-6.86(\mathrm{~m}, 6 \mathrm{H})$, 5.39 (s, 1H), 2.66 (s, 12H). ${ }^{13} \mathbf{C}$ NMR (100 MHz, $\left.\mathbf{C D C l}_{3}\right) \delta$ 156.39, 152.48, 147.31, 144.42, 136.81, 135.38, 132.24, 130.31, 129.33, 129.28, 128.60, 128.31, 128.13, 126.26, 124.92, 120.97, 118.91, 115.51, 111.22, 55.27, 45.87. HRMS (ESI) calculated $[\mathrm{M}+\mathrm{H}]^{+}$for $\mathrm{C}_{51} \mathrm{H}_{45} \mathrm{O}_{2} \mathrm{~N}_{4}$ : 745.3537, found: 745.3528. FTIR ( $\left.\mathbf{c m}^{-1}\right)$ : 2976, 2892, 1639, 1391, 1321, 1220, 1083, 1047, 880, $768,669$.

\section{4-((2-(Dimethylamino)phenyl)(3,5-dimethylphenoxy)methyl)benzonitrile (40)}

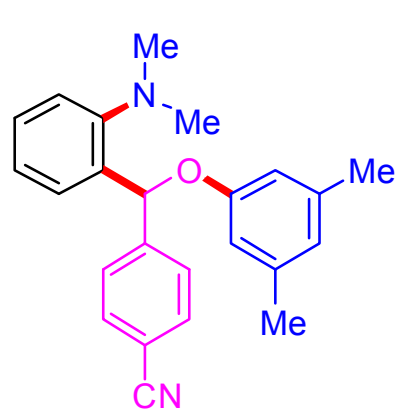

Following the general procedure $\mathbf{A}$, treatment of 2(trimethylsilyl)phenyl trifluoromethanesulfonate 1a $(0.179 \mathrm{~g}, 146 \mu \mathrm{L}$, $0.60 \mathrm{mmol})$ and 4-formylbenzonitrile $\mathbf{2 a}(0.131 \mathrm{~g}, 1.0 \mathrm{mmol})$ with $N$ $N, N, 3,5$-tetramethylaniline $30(0.075 \mathrm{~g}, 82 \mu \mathrm{L}, 0.50 \mathrm{mmol})$ in the presence of $\mathrm{KF}(0.070 \mathrm{~g}, 1.2 \mathrm{mmol})$ and [18]-crown-6 (0.317 g, 1.2 $\mathrm{mmol})$ in THF $(2.0 \mathrm{~mL})$ at $-10{ }^{\circ} \mathrm{C}$ to $\mathrm{rt}$ for $12 \mathrm{~h}$ followed by flash column chromatography (Pet. ether $/ \mathrm{EtOAc}=98 / 02)$ of the crude reaction mixture using silica gel afforded 4-((2-(dimethylamino)phenyl)(3,5-dimethylphenoxy)methyl)benzonitrile 40 as a yellow viscous oil $(0.054 \mathrm{~g}, 30 \%)$.

$\boldsymbol{R}_{\mathbf{f}}\left(\right.$ Pet. ether $/$ EtOAc $=$ 95/05): 0.50; ${ }^{1} \mathbf{H}$ NMR $\left(\mathbf{4 0 0} \mathbf{~ M H z}, \mathbf{C D C l}_{3}\right) \delta 7.62(\mathrm{~d}, J=8.2 \mathrm{~Hz}, 2 \mathrm{H})$, 7.55 (d, $J=8.3 \mathrm{~Hz}, 2 \mathrm{H}), 7.39$ (d, $J=7.7 \mathrm{~Hz}, 1 \mathrm{H}), 7.34-7.30$ (m, 1H), 7.27-7.25 (m, 1H), 7.12 (t, $J=7.4 \mathrm{~Hz}, 1 \mathrm{H}), 6.93(\mathrm{~s}, 1 \mathrm{H}), 6.62-6.59(\mathrm{~m}, 3 \mathrm{H}), 2.70(\mathrm{~s}, 6 \mathrm{H}), 2.25(\mathrm{~s}, 6 \mathrm{H}) .{ }^{13} \mathbf{C}$ NMR (100 MHz, $\left.\mathbf{C D C l}_{3}\right) \delta 157.97,152.60,147.56,139.22,135.54,132.23,129.26,128.85,128.15,124.93$, 123.04, 120.78, 118.98, 113.49, 111.18, 74.88, 45.91, 21.54. HRMS (ESI) calculated $[\mathrm{M}+\mathrm{H}]^{+}$ for $\mathrm{C}_{24} \mathrm{H}_{25} \mathrm{ON}_{2}: 357.1961$, found: 357.1958. FTIR $\left(\mathbf{c m}^{-1}\right): 3021,2934,2788,2231,1598,1487$, $1216,1049,767,671$.

\section{4-((Benzo[d][1,3]dioxol-5-yloxy)(2-(dimethylamino)phenyl)methyl)benzonitrile (4p)}

Following the general procedure $\mathbf{A}$, treatment of 2-(trimethylsilyl)phenyl trifluoromethane sulfonate 1a $(0.090 \mathrm{~g}, 73 \mu \mathrm{L}, 0.30 \mathrm{mmol})$ and 4-formylbenzonitrile 2a $(0.066 \mathrm{~g}, 0.50 \mathrm{mmol})$ with $N, N$-dimethylbenzo $[d][1,3]$ dioxol-5-amine $3 \mathbf{p}(0.041 \mathrm{~g}, 0.25 \mathrm{mmol})$ in the presence of $\mathrm{KF}$ 
$(0.035 \mathrm{~g}, 0.60 \mathrm{mmol})$ and 18 -crown-6 $(0.159 \mathrm{~g}, 0.60 \mathrm{mmol})$ in THF $(1.0 \mathrm{~mL})$ at $-10{ }^{\circ} \mathrm{C}$ to $\mathrm{rt}$ for $12 \mathrm{~h}$ followed by flash column chromatography of the crude reaction mixture using silica gel afforded two product as follows 4-((benzo[d][1,3]dioxol-5-yloxy)(2-(dimethylamino)phenyl) methyl)benzonitrile $4 \mathbf{p}$ as a colourless viscous oil ( $0.030 \mathrm{~g}, 32 \%$ yield).

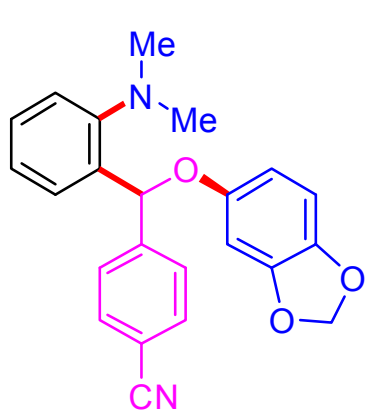

$\boldsymbol{R}_{\mathbf{f}}($ Pet. ether $/$ EtOAc $=80 / 20): 0.66 ;{ }^{1} \mathbf{H}$ NMR (400 MHz, $\left.\mathbf{C D C l}_{3}\right) \delta$ $7.61(\mathrm{~d}, J=8.3 \mathrm{~Hz}, 2 \mathrm{H}), 7.52(\mathrm{~d}, J=8.3 \mathrm{~Hz}, 2 \mathrm{H}), 7.36\left(\mathrm{dd}, J_{l}=7.7\right.$ $\left.\mathrm{Hz}, J_{2}=1.4 \mathrm{~Hz}, 1 \mathrm{H}\right), 7.32-7.28(\mathrm{~m}, 1 \mathrm{H}), 7.25-7.23(\mathrm{~m}, 1 \mathrm{H}), 7.13-7.09$ $(\mathrm{m}, 1 \mathrm{H}), 6.81(\mathrm{~s}, 1 \mathrm{H}), 6.62(\mathrm{~d}, J=8.5 \mathrm{~Hz}, 1 \mathrm{H}), 6.55(\mathrm{~d}, J=2.5 \mathrm{~Hz}$, $1 \mathrm{H}), 6.36\left(\mathrm{dd}, J_{1}=8.5 \mathrm{~Hz}, J_{2}=2.5 \mathrm{~Hz}, 1 \mathrm{H}\right), 5.87(\mathrm{~s}, 2 \mathrm{H}), 2.64(\mathrm{~s}, 6 \mathrm{H})$.

${ }^{13}$ C NMR (100 MHz, $\left.\mathbf{C D C l}_{3}\right) \delta$ 153.32, 152.68, 148.28, 147.33, 142.03, 135.44, 132.28, 129.37, 128.73, 128.08, 124.95, 120.99, 118.93, 111.29, 108.08, 107.66, 101.27, 99.15, 76.33, 45.90. HRMS (ESI) calculated $[\mathrm{M}+\mathrm{H}]^{+}$for $\mathrm{C}_{23} \mathrm{H}_{21} \mathrm{O}_{3} \mathrm{~N}_{2}: 373.1547$, found: 373.1548. FTIR (cm $\left.{ }^{-1}\right): 3020,2938,2883,2785,2230,1611,1490,1355,1212,1185,1134$, 1097, 1037, 939, 767, 667.

\section{$N, N$-Dimethyl-2-(phenoxy(phenyl)methyl)aniline (4q)}

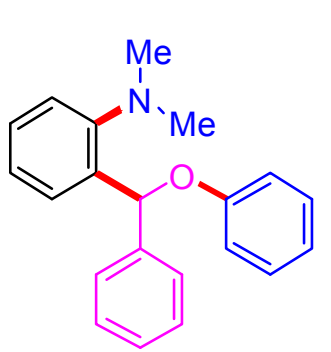

Following the general procedure $\mathbf{B}$, treatment of 2-(trimethylsilyl) phenyltrifluoromethanesulfonate 1a $(0.179 \mathrm{~g}, 146 \mu \mathrm{L}, 0.60 \mathrm{mmol})$ and benzaldehyde $\mathbf{2 b}(0.106 \mathrm{~g}, 101 \mu \mathrm{L}, 1.0 \mathrm{mmol})$ with $N, N$-dimethylaniline $\mathbf{3 b}$ $(0.061 \mathrm{~g}, 64 \mu \mathrm{L}, 0.50 \mathrm{mmol})$ in the presence of $\mathrm{KF}(0.070 \mathrm{~g}, 1.20 \mathrm{mmol})$ and 18-crown-6 $(0.317 \mathrm{~g}, 1.20 \mathrm{mmol})$ in THF $(2.0 \mathrm{~mL})$ at $-10^{\circ} \mathrm{C}$ to $\mathrm{rt}$ for 12 h followed by flash column chromatography $($ Pet. ether/EtOAc $=99 / 01)$ of the crude reaction mixture using silica gel afforded $N, N$-dimethyl-2-(phenoxy(phenyl)methyl)aniline $\mathbf{4 q}$ as a colourless viscous oil ( $0.082 \mathrm{~g}, 54 \%$ yield $)$.

$\boldsymbol{R}_{\mathbf{f}}($ Pet. ether $/$ EtOAc $=97 / 03): 0.46 ;{ }^{1} \mathbf{H}$ NMR (400 MHz, $\left.\mathbf{C D C l}_{3}\right) \delta 7.58\left(\mathrm{dd}, J_{1}=7.7 \mathrm{~Hz}, J_{2}=\right.$ $1.4 \mathrm{~Hz}, 1 \mathrm{H}), 7.53(\mathrm{~d}, J=7.4 \mathrm{~Hz}, 2 \mathrm{H}), 7.43-7.27(\mathrm{~m}, 7 \mathrm{H}), 7.18(\mathrm{t}, J=7.4 \mathrm{~Hz}, 1 \mathrm{H}), 7.08-7.06(\mathrm{~m}$, 2H), $7.01(\mathrm{~s}, 1 \mathrm{H}), 6.98-6.95(\mathrm{~m}, 1 \mathrm{H}), 2.75(\mathrm{~s}, 6 \mathrm{H}) .{ }^{13} \mathbf{C}$ NMR (100 MHz, CDCl $) \delta 158.47$, 152.66, 141.60, 136.08, 129.38, 129.11, 128.76, 128.44, 127.75, 127.55, 124.50, 120.67, 120.41, 115.86, 75.71, 45.89. HRMS (ESI) calculated $[\mathrm{M}+\mathrm{H}]^{+}$for $\mathrm{C}_{21} \mathrm{H}_{22} \mathrm{ON}$ : 304.1696, found: 304.1689. FTIR (cm $\left.{ }^{-1}\right): 3020,2940,2786,1594,1492,1221,764$. 


\section{2-((4-Bromophenyl)(phenoxy)methyl)- $N, N$-dimethylaniline (4r)}

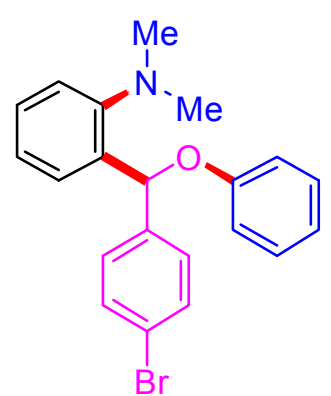

Following the general procedure B, treatment of 2-(trimethylsilyl)phenyl trifluoromethanesulfonate $1 \mathrm{a}(0.179 \mathrm{~g}, 146 \mu \mathrm{L}, 0.60 \mathrm{mmol})$ and 4bromobenzaldehyde 2 c $(0.185 \mathrm{~g}, 1.0 \mathrm{mmol})$ with $N, N$-dimethylaniline $\mathbf{3 b}$ $(0.061 \mathrm{~g}, 64 \mu \mathrm{L}, 0.50 \mathrm{mmol})$ in the presence of $\mathrm{KF}(0.070 \mathrm{~g}, 1.20 \mathrm{mmol})$ and [18]-crown-6 $(0.317 \mathrm{~g}, 1.20 \mathrm{mmol})$ in THF $(2.0 \mathrm{~mL})$ at $-10^{\circ} \mathrm{C}$ to $\mathrm{rt}$ for $12 \mathrm{~h}$ followed by flash column chromatography (Pet. ether $/$ EtOAc $=$ 98/02) of the crude reaction mixture using silica gel afforded 2-((4-bromophenyl) (phenoxy)methyl)- $N, N$-dimethylaniline $4 \mathrm{r}$ as a yellow viscous oil (0.166 g, 87\%).

$\boldsymbol{R}_{\mathbf{f}}($ Pet. ether $/$ EtOAc $=97 / 03)$ : 0.50; ${ }^{1} \mathbf{H}$ NMR (400 MHz, $\left.\mathbf{C D C l}_{3}\right) \delta$ 7.53-7.51 (m, 3H), $7.39(\mathrm{~d}$, $J=8.6 \mathrm{~Hz}, 2 \mathrm{H}), 7.36-7.34(\mathrm{~m}, 1 \mathrm{H}), 7.31-7.26(\mathrm{~m}, 3 \mathrm{H}), 7.19-7.15(\mathrm{~m}, 1 \mathrm{H}), 7.05-7.02(\mathrm{~m}, 2 \mathrm{H})$, 6.98-6.94 (m, 2H), 2.73 (s, 6H). ${ }^{13} \mathbf{C}$ NMR (100 MHz, CDCl $) \delta 158.20,152.60,140.83,135.75$, $131.55,129.45,129.41,128.99,128.83,124.67,121.51,120.93,120.62,115.85,75.26,45.88$. HRMS (ESI) calculated $[\mathrm{M}+\mathrm{H}]^{+}$for $\mathrm{C}_{21} \mathrm{H}_{21} \mathrm{BrON}$ : 382.0801 , found: 382.0798 . FTIR $\left(\mathbf{c m}^{-1}\right)$ : 3019, 2940, 2786, 1592, 1490, 1222, 1014, 764, 677.

\section{2-((4-Chlorophenyl)(phenoxy)methyl)- $N, N$-dimethylaniline (4s)}

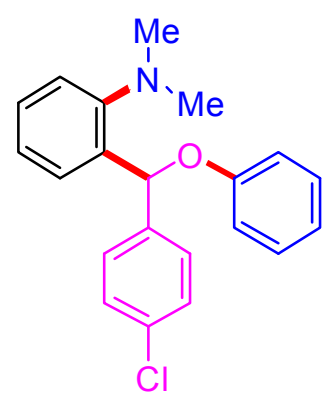

Following the general procedure B, treatment of 2-(trimethylsilyl) phenyltrifluoromethanesulfonate 1a $(0.179 \mathrm{~g}, 146 \mu \mathrm{L}, 0.60 \mathrm{mmol})$ and 4chlorobenzaldehyde 2 d $(0.140 \mathrm{~g}, 1.0 \mathrm{mmol})$ with $N, N$-dimethylaniline $3 \mathbf{b}$ $(0.061 \mathrm{~g}, 64 \mu \mathrm{L}, 0.50 \mathrm{mmol})$ in the presence of $\mathrm{KF}(0.070 \mathrm{~g}, 1.20 \mathrm{mmol})$ and 18-crown-6 $(0.317 \mathrm{~g}, 1.20 \mathrm{mmol})$ in THF $(2.0 \mathrm{~mL})$ at $-10^{\circ} \mathrm{C}$ to $\mathrm{rt}$ for $12 \mathrm{~h}$ followed by flash column chromatography $($ Pet. ether/EtOAc $=99 / 01)$ of the crude reaction mixture using silica gel afforded 2-((4-chlorophenyl)(phenoxy)methyl)$N, N$-dimethylaniline $4 \mathbf{s}$ as a colourless viscous oil (0.132 g, 78\% yield).

$\boldsymbol{R}_{\mathbf{f}}($ Pet. ether $/$ EtOAc $=97 / 03)$ : 0.50; ${ }^{1} \mathbf{H}$ NMR (400 MHz, $\left.\mathbf{C D C l}_{3}\right) \delta 7.44\left(\mathrm{dd}, J_{1}=7.7 \mathrm{~Hz}, J_{2}=\right.$ $1.4 \mathrm{~Hz}, 1 \mathrm{H}), 7.37$ (d, $J=8.5 \mathrm{~Hz}, 2 \mathrm{H}), 7.31-7.28$ (m, 3H), 7.24-7.20 (m, 3H), 7.13-7.09 (m, 1H), $6.96(\mathrm{~d}, J=7.9 \mathrm{~Hz}, 2 \mathrm{H}), 6.92-6.88(\mathrm{~m}, 2 \mathrm{H}), 2.66(\mathrm{~s}, 6 \mathrm{H}) .{ }^{13} \mathbf{C}$ NMR (100 MHz, CDCl$) \delta$ $158.23,152.64,140.30,135.81,133.35,129.46,129.10,129.00,128.85,128.63,124.68,120.92$, 120.64, 115.87, 75.22, 45.90. HRMS (ESI) calculated $[\mathrm{M}+\mathrm{H}]^{+}$for $\mathrm{C}_{21} \mathrm{H}_{21} \mathrm{ONCl}$ : 338.1306, found: 338.1300. FTIR ( $\left.\mathbf{c m}^{-1}\right)$ : 3018, 2939, 2832, 2785, 1592, 1490, 1224, 1091, 1015, 761. 


\section{2-((4-Fluorophenyl)(phenoxy)methyl)- $N, N$-dimethylaniline (4t)}

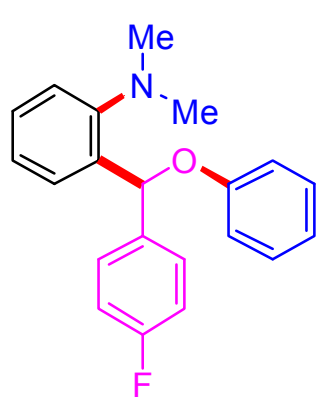

Following the general procedure $\mathbf{B}$, treatment of 2-(trimethylsilyl)phenyl trifluoromethanesulfonate 1a $(0.179 \mathrm{~g}, 146 \mu \mathrm{L}, 0.60 \mathrm{mmol})$ and $4-$ fluorobenzaldehyde $2 \mathrm{e}(0.124 \mathrm{~g}, 1.0 \mathrm{mmol})$ with $N, N$-dimethylaniline $\mathbf{3 b}$ $(0.061 \mathrm{~g}, 64 \mu \mathrm{L}, 0.50 \mathrm{mmol})$ in the presence of $\mathrm{KF}(0.070 \mathrm{~g}, 1.20 \mathrm{mmol})$ and [18]-crown-6 $(0.317 \mathrm{~g}, 1.20 \mathrm{mmol})$ in THF $(2.0 \mathrm{~mL})$ at $-10{ }^{\circ} \mathrm{C}$ to $\mathrm{rt}$ for $12 \mathrm{~h}$ followed by flash column chromatography (Pet. ether $/$ EtOAc $=99 / 01)$

of the crude reaction mixture using silica gel afforded 2-((4-fluorophenyl)(phenoxy)methyl)$N, N$-dimethylaniline $4 \mathrm{t}$ as a yellow viscous oil $(0.114 \mathrm{~g}, 71 \%)$.

$\boldsymbol{R}_{\mathbf{f}}($ Pet. ether $/$ EtOAc $=97 / 03): 0.44 ;{ }^{1} \mathbf{H}$ NMR $\left(\mathbf{4 0 0} \mathbf{~ M H z}, \mathbf{C D C l}_{3}\right) \delta 7.51-7.49(\mathrm{~m}, 1 \mathrm{H}), 7.45-$ $7.42(\mathrm{~m}, 2 \mathrm{H}), 7.34-7.30(\mathrm{~m}, 1 \mathrm{H}), 7.27-7.21(\mathrm{~m}, 3 \mathrm{H}), 7.14(\mathrm{t}, J=7.4 \mathrm{~Hz}, 1 \mathrm{H}), 7.05(\mathrm{t}, J=8.7$ $\mathrm{Hz}, 2 \mathrm{H}), 7.00-6.98(\mathrm{~m}, 2 \mathrm{H}), 6.94-6.91(\mathrm{~m}, 2 \mathrm{H}), 2.68(\mathrm{~s}, 6 \mathrm{H}) .{ }^{13} \mathbf{C} \mathbf{~ N M R}\left(\mathbf{1 0 0} \mathbf{~ M H z}, \mathbf{C D C l}_{3}\right) \delta$ $162.24(\mathrm{~d}, J=246.0 \mathrm{~Hz}), 158.29,152.61,136.95$ (d, $J=2.9 \mathrm{~Hz}), 135.97,129.54,129.43$, 128.91, 128.78, 124.63, 120.84, 120.60, 115.87, 115.31 (d, $J=21.5 \mathrm{~Hz}), 75.23,45.87$. HRMS (ESI) calculated $[\mathrm{M}+\mathrm{H}]^{+}$for $\mathrm{C}_{21} \mathrm{H}_{21} \mathrm{FON}: 322.1602$, found: 322.1593 . FTIR $\left(\mathbf{c m}^{-1}\right)$ : 3020, 2942 , 2787, 1596, 1498, 1222, 1018, 765, 675.

\section{Methyl-4-((2-(dimethylamino)phenyl)(phenoxy)methyl)benzoate (4u)}

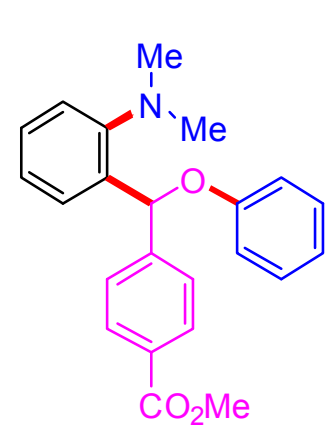

Following the general procedure A, treatment of 2-(trimethylsilyl)phenyl trifluoromethanesulfonate 1a $(0.179 \mathrm{~g}, 146 \mu \mathrm{L}, 0.60 \mathrm{mmol})$ and methyl 4formylbenzoate $2 \mathbf{f}(0.164 \mathrm{~g}, 1.0 \mathrm{mmol})$ with $N, N$-dimethylaniline $\mathbf{3 b}$ $(0.061 \mathrm{~g}, 64 \mu \mathrm{L}, 0.50 \mathrm{mmol})$ in the presence of $\mathrm{KF}(0.070 \mathrm{~g}, 1.20 \mathrm{mmol})$ and [18]-crown-6 (0.317 g, $1.20 \mathrm{mmol})$ in THF $(2.0 \mathrm{~mL})$ at $-10{ }^{\circ} \mathrm{C}$ to $\mathrm{rt}$ for $12 \mathrm{~h}$ followed by flash column chromatography (Pet. ether /EtOAc $=$

98/02) of the crude reaction mixture using silica gel afforded methyl-4-((2(dimethylamino)phenyl)(phenoxy)methyl)benzoate $4 \mathbf{u}$ as a colourless viscous oil $(0.126 \mathrm{~g}, 70 \%)$. $\boldsymbol{R}_{\mathbf{f}}\left(\right.$ Pet. ether $/$ EtOAc $=$ 95/05): 0.44; ${ }^{1} \mathbf{H}$ NMR $\left(\mathbf{4 0 0} \mathbf{~ M H z}, \mathbf{C D C l}_{\mathbf{3}}\right) \delta 8.05(\mathrm{~d}, J=8.3 \mathrm{~Hz}, 2 \mathrm{H})$, $7.56(\mathrm{~d}, J=8.3 \mathrm{~Hz}, 2 \mathrm{H}), 7.47$ (dd, $J=7.7,1.3 \mathrm{~Hz}, 1 \mathrm{H}), 7.35-7.31(\mathrm{~m}, 1 \mathrm{H}), 7.28-7.23(\mathrm{~m}, 3 \mathrm{H})$, $7.14(\mathrm{t}, J=7.3 \mathrm{~Hz}, 1 \mathrm{H}), 7.02-7.00(\mathrm{~m}, 3 \mathrm{H}), 6.94(\mathrm{t}, J=7.3 \mathrm{~Hz}, 1 \mathrm{H}), 3.93(\mathrm{~s}, 3 \mathrm{H}), 2.70(\mathrm{~s}, 6 \mathrm{H})$. ${ }^{13}$ C NMR (100 MHz, $\left.\mathbf{C D C l}_{3}\right) \delta$ 166.99, 158.20, 152.68, 146.99, 135.81, 129.77, 129.46, 129.30, $129.07,128.97,127.49,124.74,121.00,120.74,115.90,75.58,52.14,45.88$. HRMS (ESI) 
calculated $[\mathrm{M}+\mathrm{H}]^{+}$for $\mathrm{C}_{23} \mathrm{H}_{24} \mathrm{O}_{3} \mathrm{~N}$ : 362.1751, found: 362.1744. FTIR (cm $\left.{ }^{-1}\right): 3023,2937,1716$, $1597,1285,1219,1171,1022,768,673$.

\section{$N, N$-Dimethyl-2-(phenoxy(4-(trifluoromethyl)phenyl)methyl)aniline (4v)}

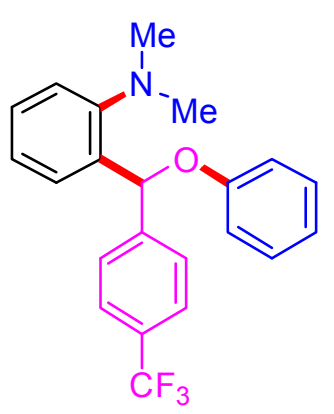

Following the general procedure B, treatment of 2-(trimethylsilyl)phenyl trifluoromethanesulfonate $1 \mathrm{a}(0.179 \mathrm{~g}, 146 \mu \mathrm{L}, 0.60 \mathrm{mmol})$ and 4(trifluoromethyl)benzaldehyde $\mathbf{2 g}(0.174 \mathrm{~g}, 1.0 \mathrm{mmol})$ with $N, N-$ dimethylaniline $3 \mathbf{b}(0.061 \mathrm{~g}, 64 \mu \mathrm{L}, 0.50 \mathrm{mmol})$ in the presence of $\mathrm{KF}$ $(0.070 \mathrm{~g}, 1.20 \mathrm{mmol})$ and [18]-crown-6 (0.317 g, $1.2 \mathrm{mmol})$ in THF (2.0 $\mathrm{mL}$ ) at $-10^{\circ} \mathrm{C}$ to $\mathrm{rt}$ for $12 \mathrm{~h}$ followed by flash column chromatography (Pet. ether $/$ EtOAc $=99 / 01)$ of the crude reaction mixture using silica gel afforded $N, N$-dimethyl2-(phenoxy(4-(trifluoromethyl)phenyl)methyl)aniline $4 \mathbf{v}$ as a yellow solid $(0.146 \mathrm{~g}, 79 \%)$.

$\boldsymbol{R}_{\mathbf{f}}\left(\right.$ Pet. ether $/$ EtOAc = 97/03): 0.48; ${ }^{1} \mathbf{H}$ NMR (400 MHz, $\left.\mathbf{C D C l}_{3}\right) \delta$ 7.66-7.61 (m, 4H), $7.49(\mathrm{~d}$, $J=7.8 \mathrm{~Hz}, 1 \mathrm{H}), 7.38-7.34(\mathrm{~m}, 1 \mathrm{H}), 7.32-7.27(\mathrm{~m}, 3 \mathrm{H}), 7.17(\mathrm{t}, J=7.4 \mathrm{~Hz}, 1 \mathrm{H}), 7.05-7.03(\mathrm{~m}$, 3H), 6.98 (t, $J=7.3 \mathrm{~Hz}, 1 \mathrm{H}), 2.73$ (s, 6H). ${ }^{13} \mathbf{C}$ NMR (100 MHz, CDCl $) \delta 158.17,152.71$, 145.94, 135.74, 129.54, $129.05(\mathrm{~d}, J=28.5 \mathrm{~Hz}), 127.85,125.44(\mathrm{~d}, J=3.5 \mathrm{~Hz}), 124.85,121.12$, 120.82, 115.91, 75.38, 45.92. HRMS (ESI) calculated $[\mathrm{M}+\mathrm{H}]^{+}$for $\mathrm{C}_{22} \mathrm{H}_{21} \mathrm{~F}_{3} \mathrm{ON}$ : 372.1570, found: 372.1566. FTIR (cm $\left.{ }^{-1}\right)$ : 3020, 2940, 2788, 1593, 1491, 1325, 1220, 1021, 768, 675.

\section{$N, N$-Dimethyl-2-((4-nitrophenyl)(phenoxy)methyl)aniline (4w)}

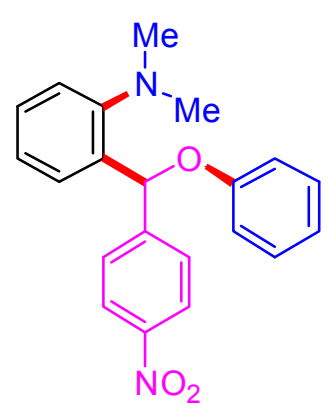

Following the general procedure A, treatment of 2-(trimethylsilyl) phenyltrifluoromethanesulfonate 1a $(0.179 \mathrm{~g}, 146 \mu \mathrm{L}, 0.60 \mathrm{mmol})$ and 4nitrobenzaldehyde $\mathbf{2 h}(0.151 \mathrm{~g}, 1.0 \mathrm{mmol})$ with $N, N$-dimethylaniline $\mathbf{3 b}$ $(0.061 \mathrm{~g}, 64 \mu \mathrm{L}, 0.50 \mathrm{mmol})$ in the presence of $\mathrm{KF}(0.070 \mathrm{~g}, 1.20 \mathrm{mmol})$ and 18-crown-6 (0.317 $\mathrm{g}, 1.20 \mathrm{mmol})$ in THF $(2.0 \mathrm{~mL})$ at $-10{ }^{\circ} \mathrm{C}$ to $\mathrm{rt}$ for $12 \mathrm{~h}$ followed by flash column chromatography (Pet. ether/EtOAc $=98 / 02)$ of the crude reaction mixture using silica gel afforded $N, N$-dimethyl-2-((4-nitrophenyl)(phen oxy)methyl)aniline $4 \mathbf{w}$ as a yellow viscous oil ( $0.140 \mathrm{~g}, 80 \%$ yield).

$\boldsymbol{R}_{\mathbf{f}}($ Pet. ether $/$ EtOAc $=95 / 05): 0.63 ;{ }^{1} \mathbf{H}$ NMR (400 MHz, $\left.\mathbf{C D C l}_{3}\right) \delta 8.21(\mathrm{~d}, J=8.7 \mathrm{~Hz}, 2 \mathrm{H})$, $7.65(\mathrm{~d}, J=8.7 \mathrm{~Hz}, 2 \mathrm{H}), 7.44\left(\mathrm{dd}, J_{1}=7.7 \mathrm{~Hz}, J_{2}=1.3 \mathrm{~Hz}, 1 \mathrm{H}\right), 7.37-7.32(\mathrm{~m}, 1 \mathrm{H}), 7.30-7.25$ $(\mathrm{m}, 3 \mathrm{H}), 7.17-7.13(\mathrm{~m}, 1 \mathrm{H}), 7.03-6.94(\mathrm{~m}, 4 \mathrm{H}), 2.71(\mathrm{~s}, 6 \mathrm{H}) .{ }^{13} \mathbf{C} \mathbf{N M R}\left(\mathbf{1 0 0} \mathbf{M H z}, \mathbf{C D C l}_{3}\right) \delta$ 
$157.85,152.60,149.37,147.22,135.39,129.58,129.40,128.70,128.15,124.98,123.69,121.36$, 121.04, 115.87, 75.26, 45.88. HRMS (ESI) calculated $[\mathrm{M}+\mathrm{H}]^{+}$for $\mathrm{C}_{21} \mathrm{H}_{21} \mathrm{O}_{3} \mathrm{~N}_{2}: 349.1547$, found: 349.1546. FTIR ( $\left.\mathbf{c m}^{-1}\right)$ : 3022, 2940, 2864, 2833, 2786, 1595, 1522, 1492, 1346, 1225 , 1022,759 .

\section{2-((3-Bromophenyl)(phenoxy)methyl)- $N, N$-dimethylaniline (4x)}

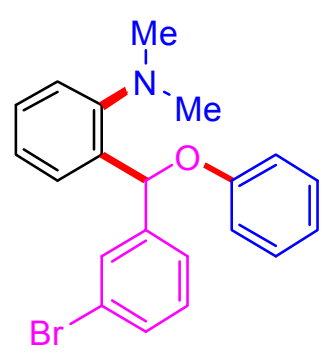

Following the general procedure $\mathbf{B}$, treatment of 2-(trimethylsilyl)phenyl trifluoromethanesulfonate 1a $(0.179 \mathrm{~g}, 146 \mu \mathrm{L}, 0.60 \mathrm{mmol})$ and 3bromobenzaldehyde $2 \mathbf{i}(0.185 \mathrm{~g}, 1.0 \mathrm{mmol})$ with $N, N$-dimethylaniline $\mathbf{3 b}$ $(0.061 \mathrm{~g}, 64 \mu \mathrm{L}, 0.50 \mathrm{mmol})$ in the presence of $\mathrm{KF}(0.070 \mathrm{~g}, 1.20 \mathrm{mmol})$ and [18]-crown-6 $(0.317 \mathrm{~g}, 1.20 \mathrm{mmol})$ in THF $(2.0 \mathrm{~mL})$ at $-10{ }^{\circ} \mathrm{C}$ to $\mathrm{rt}$ for $12 \mathrm{~h}$ followed by flash column chromatography (Pet. ether $/ \mathrm{EtOAc}=$ 98/02) of the crude reaction mixture using silica gel afforded 2-((3-bromophenyl)(phenoxy) methyl)- $N, N$-dimethylaniline $4 \mathbf{x}$ as a colourless viscous oil $(0.135 \mathrm{~g}, 70 \%)$.

$\boldsymbol{R}_{\mathbf{f}}($ Pet. ether $/$ EtOAc $=97 / 03): 0.43 ;{ }^{1} \mathbf{H}$ NMR $\left(400 \mathbf{~ M H z}, \mathbf{C D C l}_{3}\right) \delta 7.70(\mathrm{~s}, 1 \mathrm{H}), 7.52\left(\mathrm{dd}, J_{1}=\right.$ 7.8, $\left.J_{2}=1.4 \mathrm{~Hz}, 1 \mathrm{H}\right), 7.44$ (t, $\left.J=8.3 \mathrm{~Hz}, 2 \mathrm{H}\right), 7.37-7.33(\mathrm{~m}, 1 \mathrm{H}), 7.30-7.22(\mathrm{~m}, 4 \mathrm{H}), 7.19-7.15$ (m, 1H), 7.03 (d, $J=7.9 \mathrm{~Hz}, 2 \mathrm{H}), 6.98-6.95$ (m, 2H), 2.72 (s, 6H). ${ }^{13} \mathbf{C}$ NMR (100 MHz, $\left.\mathbf{C D C l}_{3}\right) \delta 158.15,152.60,144.16,135.68,130.66,130.64,130.01,129.46,129.04,128.81$, 126.16, 124.77, 122.62, 120.98, 120.72, 115.87, 75.22, 45.90. HRMS (ESI) calculated $[\mathrm{M}+\mathrm{H}]^{+}$ for $\mathrm{C}_{21} \mathrm{H}_{21} \mathrm{BrON}$ : 382.0801, found: 382.0798. FTIR $\left(\mathbf{c m}^{-1}\right)$ : 3064, 3018, 2941, 2866, 2831, 2785, 1724, 1590, 1487, 1297, 1225, 1017, 765, 684.

\section{$N, N$-Dimethyl-2-((3-nitrophenyl)(phenoxy)methyl)aniline (4y)}

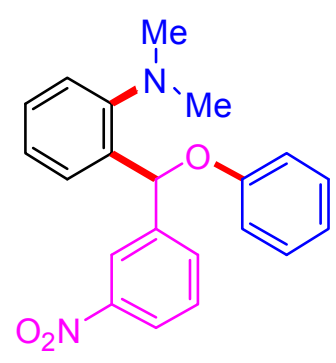

Following the general procedure A, treatment of 2-(trimethylsilyl)phenyl trifluoromethanesulfonate 1a $(0.179 \mathrm{~g}, 146 \mu \mathrm{L}, 0.60 \mathrm{mmol})$ and $3-$ nitrobenzaldehyde $\mathbf{2} \mathbf{j}(0.151 \mathrm{~g}, 1.0 \mathrm{mmol})$ with $N, N$-dimethylaniline $\mathbf{3 b}$ $(0.061 \mathrm{~g}, 64 \mu \mathrm{L}, 0.50 \mathrm{mmol})$ in the presence of $\mathrm{KF}(0.070 \mathrm{~g}, 1.20 \mathrm{mmol})$ and [18]-crown-6 $(0.317 \mathrm{~g}, 1.20 \mathrm{mmol})$ in THF $(2.0 \mathrm{~mL})$ at $-10{ }^{\circ} \mathrm{C}$ to $\mathrm{rt}$ for $12 \mathrm{~h}$ followed by flash column chromatography (Pet. ether $/ \mathrm{EtOAc}=$ 98/02) of the crude reaction mixture using silica gel afforded $N, N$-dimethyl-2-((3nitrophenyl)(phenoxy)methyl)aniline $\mathbf{4 y}$ as a yellow viscous oil $(0.119 \mathrm{~g}, 68 \%)$. 
$\boldsymbol{R}_{\mathbf{f}}($ Pet. ether $/$ EtOAc $=95 / 05): 0.60 ;{ }^{1} \mathbf{H}$ NMR (400 MHz, $\left.\mathbf{C D C l}_{3}\right) \delta 8.38(\mathrm{~s}, 1 \mathrm{H}), 8.35\left(\mathrm{dd}, J_{1}=\right.$ $\left.8.2, J_{2}=1.2 \mathrm{~Hz}, 1 \mathrm{H}\right), 8.82(\mathrm{~d}, J=7.7 \mathrm{~Hz}, 1 \mathrm{H}), 7.51(\mathrm{t}, J=8.2 \mathrm{~Hz}, 2 \mathrm{H}), 7.36-7.24(\mathrm{~m}, 4 \mathrm{H}), 7.18-$ $7.14(\mathrm{~m}, 1 \mathrm{H}), 7.01(\mathrm{~s}, 2 \mathrm{H}), 6.99(\mathrm{~s}, 1 \mathrm{H}), 6.95(\mathrm{t}, J=7.3 \mathrm{~Hz}, 1 \mathrm{H}), 2.70(\mathrm{~s}, 6 \mathrm{H}) .{ }^{13} \mathrm{C}$ NMR (100 MHz, $\left.\mathbf{C D C l}_{3}\right) \delta 157.84,152.56,148.41,144.24,135.52,133.42,129.58,129.37,128.37,125.12$, 122.57, 122.56, 121.35, 121.18, 115.94, 75.23, 45.90. HRMS (ESI) calculated $[\mathrm{M}+\mathrm{H}]^{+}$for $\mathrm{C}_{21} \mathrm{H}_{21} \mathrm{O}_{3} \mathrm{~N}_{2}$ : 349.1547, found: 349.1545. FTIR (cm $\left.{ }^{-1}\right): 3022,2940,1593,1531,1490,1350$, $1221,1028,765,679$.

\section{2-((2-Fluorophenyl)(phenoxy)methyl)- $N, N$-dimethylaniline (4z)}

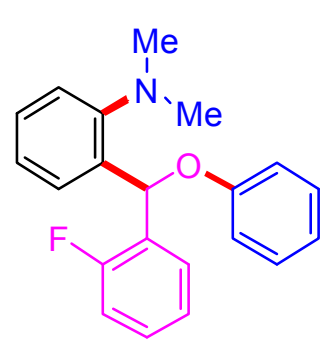

Following the general procedure B, treatment of 2-(trimethylsilyl)phenyl trifluoromethanesulfonate $1 \mathrm{a}(0.179 \mathrm{~g}, 146 \mu \mathrm{L}, 0.60 \mathrm{mmol})$ and 2fluorobenzaldehyde $\mathbf{2 k}(0.124 \mathrm{~g}, 1.0 \mathrm{mmol})$ with $N, N$-dimethylaniline $\mathbf{3 b}$ $(0.061 \mathrm{~g}, 64 \mu \mathrm{L}, 0.50 \mathrm{mmol})$ in the presence of $\mathrm{KF}(0.070 \mathrm{~g}, 1.20 \mathrm{mmol})$ and [18]-crown-6 $(0.317 \mathrm{~g}, 1.20 \mathrm{mmol})$ in THF $(2.0 \mathrm{~mL})$ at $-10{ }^{\circ} \mathrm{C}$ to $\mathrm{rt}$ for $12 \mathrm{~h}$ followed by flash column chromatography (Pet. ether $/$ EtOAc $=$ 98/02) of the crude reaction mixture using silica gel afforded 2-((2-fluorophenyl)(phenoxy) methyl)- $N, N$-dimethylaniline $\mathbf{4 z}$ as a colourless viscous oil $(0.126 \mathrm{~g}, 78 \%)$.

$\boldsymbol{R}_{\mathbf{f}}($ Pet. ether $/$ EtOAc $=97 / 03): 0.44 ;{ }^{1} \mathbf{H}$ NMR (400 $\left.\mathbf{M H z}, \mathbf{C D C l}_{3}\right) \delta 7.51(\mathrm{~d}, J=7.7 \mathrm{~Hz}, 1 \mathrm{H})$, $7.44(\mathrm{t}, J=7.5 \mathrm{~Hz}, 1 \mathrm{H}), 7.35-7.20(\mathrm{~m}, 6 \mathrm{H}), 7.15-7.05(\mathrm{~m}, 3 \mathrm{H}), 6.99-6.97(\mathrm{~m}, 2 \mathrm{H}), 6.92(\mathrm{t}, J=$ $7.3 \mathrm{~Hz}, 1 \mathrm{H}), 2.64$ (s, 6H). ${ }^{13} \mathbf{C}$ NMR (100 MHz, CDCl $\mathbf{~ M ~}_{3} 160.81(\mathrm{~d}, J=248.1 \mathrm{~Hz}), 158.24$, 152.97, 134.98, 129.45, 129.14 (d, $J=3.4 \mathrm{~Hz}), 129.0$ (d, $J=7.8 \mathrm{~Hz}), 124.34,124.07$ (d, $J=3.2$ Hz), 120.90, 120.87, 115.80, 115.6 (d, $J=21.7 \mathrm{~Hz}), 70.30,45.65$. HRMS (ESI) calculated $[\mathrm{M}+\mathrm{H}]^{+}$for $\mathrm{C}_{21} \mathrm{H}_{21} \mathrm{FON}: 322.1602$, found: 322.1599. FTIR $\left(\mathbf{c m}^{\mathbf{- 1}}\right)$ : 3019, 2942, 2866, 2831, $2784,1591,1490,1458,1296,1225,1018,765,680$.

\section{$N, N$-Dimethyl-2-((2-nitrophenyl)(phenoxy)methyl)aniline (4aa)}

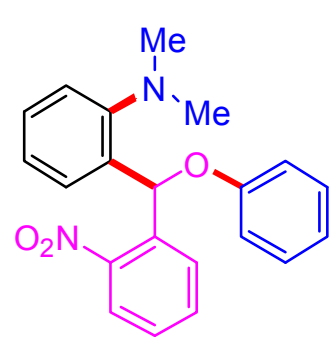

Following the general procedure A, treatment of 2-(trimethylsilyl) phenyltrifluoromethanesulfonate 1a $(0.179 \mathrm{~g}, 146 \mu \mathrm{L}, 0.60 \mathrm{mmol})$ and 2nitrobenzaldehyde 21 (0.151 g, $1.0 \mathrm{mmol})$ with $N, N$-dimethylaniline $\mathbf{3 b}$ $(0.061 \mathrm{~g}, 64 \mu \mathrm{L}, 0.50 \mathrm{mmol})$ in the presence of $\mathrm{KF}(0.070 \mathrm{~g}, 1.20 \mathrm{mmol})$ and 18 -crown-6 $(0.317 \mathrm{~g}, 1.20 \mathrm{mmol})$ in THF $(2.0 \mathrm{~mL})$ at $-10^{\circ} \mathrm{C}$ to $\mathrm{rt}$ for 
$12 \mathrm{~h}$ followed by flash column chromatography (Pet. ether/EtOAc $=99 / 01)$ of the crude reaction mixture using silica gel afforded $N, N$-dimethyl-2-((2-nitrophenyl)(phenoxy)methyl)aniline 4aa as a yellow viscous oil ( $0.134 \mathrm{~g}, 77 \%$ yield).

$\boldsymbol{R}_{\mathbf{f}}($ Pet. ether $/$ EtOAc $=95 / 05): 0.65 ;{ }^{1} \mathbf{H}$ NMR $\left(400 \mathbf{~ M H z}, \mathbf{C D C l}_{3}\right) \delta 7.95\left(\mathrm{dd}, J_{1}=8.1 \mathrm{~Hz}, J_{2}=\right.$ $1.1 \mathrm{~Hz}, 1 \mathrm{H}), 7.66\left(\mathrm{dd}, J_{I}=7.8 \mathrm{~Hz}, J_{2}=1.0 \mathrm{~Hz}, 1 \mathrm{H}\right), 7.60(\mathrm{~s}, 1 \mathrm{H}), 7.56(\mathrm{t}, J=7.7 \mathrm{~Hz}, 1 \mathrm{H}), 7.46-$ $7.42(\mathrm{~m}, 1 \mathrm{H}), 7.41-7.35(\mathrm{~m}, 2 \mathrm{H}), 7.32-7.28(\mathrm{~m}, 3 \mathrm{H}), 7.15(\mathrm{t}, J=7.6 \mathrm{~Hz}, 1 \mathrm{H}), 7.07$ (d, $J=7.9 \mathrm{~Hz}$, 2H), 6.99 (t, $J=7.3 \mathrm{~Hz}, 1 \mathrm{H}), 2.66$ (s, 6H). ${ }^{13} \mathbf{C} \mathbf{~ N M R}\left(\mathbf{1 0 0} \mathbf{~ M H z}, \mathbf{C D C l}_{3}\right) \delta$ 157.91, 153.08, 149.07, 136.28, 135.10, 132.70, 129.54, 129.39, 128.38, 128.00, 124.58, 124.57, 121.27, 115.62, 72.10, 45.36. HRMS (ESI) calculated $[\mathrm{M}+\mathrm{H}]^{+}$for $\mathrm{C}_{21} \mathrm{H}_{21} \mathrm{O}_{3} \mathrm{~N}_{2}: 349.1547$, found: 349.1543 . FTIR $\left(\mathbf{c m}^{-1}\right)$ : 3021, 2786, 1593, 1530, 1490, 1355, 1220, 759 .

\section{2-((3,4-Dichlorophenyl)(phenoxy)methyl)- $N, N$-dimethylaniline (4ab)}

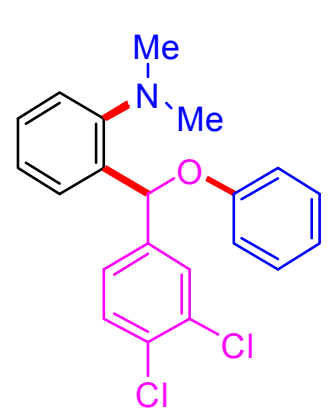

Following the general procedure $\mathbf{B}$, treatment of 2-(trimethylsilyl) phenyltrifluoromethanesulfonate 1a $(0.179 \mathrm{~g}, 146 \mu \mathrm{L}, 0.60 \mathrm{mmol})$ and $3,4-$ dichlorobenzaldehyde $\mathbf{2 m}(0.175 \mathrm{~g}, 1.0 \mathrm{mmol})$ with $N, N$-dimethylaniline 3b $(0.061 \mathrm{~g}, 64 \mu \mathrm{L}, 0.50 \mathrm{mmol})$ in the presence of $\mathrm{KF}(0.070 \mathrm{~g}, 1.20$ mmol) and 18-crown-6 $(0.317 \mathrm{~g}, 1.20 \mathrm{mmol})$ in THF $(2.0 \mathrm{~mL})$ at $-10{ }^{\circ} \mathrm{C}$ to rt for $12 \mathrm{~h}$ followed by flash column chromatography (Pet. ether/EtOAc

$=99 / 01)$ of the crude reaction mixture using silica gel afforded 2-((3,4-dichlorophenyl) (phenoxy)methyl)- $N, N$-dimethylaniline $4 \mathbf{a b}$ as a colourless viscous oil $(0.165 \mathrm{~g}, 88 \%$ yield).

$\boldsymbol{R}_{\mathbf{f}}($ Pet. ether $/$ EtOAc $=97 / 03): 0.50 ;{ }^{1} \mathbf{H}$ NMR $\left(\mathbf{4 0 0} \mathbf{~ M H z}, \mathbf{C D C l}_{3}\right) \delta 7.56(\mathrm{~d}, J=1.8 \mathrm{~Hz}, 1 \mathrm{H})$, 7.45-7.39 (m, 2H), 7.33-7.21 (m, 5H), 7.13 (t, $J=7.4 \mathrm{~Hz}, 1 \mathrm{H}), 6.96-6.90(\mathrm{~m}, 3 \mathrm{H}), 6.85(\mathrm{~s}, 1 \mathrm{H})$, 2.67 (s, 6H). ${ }^{13} \mathbf{C}$ NMR (100 MHz, $\left.\mathbf{C D C l}_{3}\right) \delta$ 158.00, 152.60, 142.24, 135.47, 132.59, 131.54, 130.44, 129.60, 129.54, 129.23, 128.64, 126.92, 124.91, 121.18, 120.88, 115.88, 74.87, 45.92. HRMS (ESI) calculated $[\mathrm{M}+\mathrm{H}]^{+}$for $\mathrm{C}_{21} \mathrm{H}_{20} \mathrm{ONCl}_{2}: 372.0916$, found: 372.0915 . FTIR ( $\left.\mathbf{c m}^{-1}\right)$ : 3020, 2937, 1593, 1486, 1221, 1031, 765, 676.

\section{$N, N$-Dimethyl-2-(naphthalen-2-yl(phenoxy)methyl)aniline (4ac)}

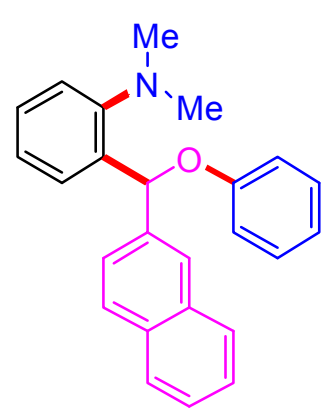

Following the general procedure $\mathbf{A}$, treatment of 2-(trimethylsilyl)phenyl trifluoromethanesulfonate 1a $(0.179 \mathrm{~g}, 146 \mu \mathrm{L}, 0.60 \mathrm{mmol})$ and 2 naphthaldehyde 2n $(0.156 \mathrm{~g}, 1.0 \mathrm{mmol})$ with $N, N$-dimethylaniline $\mathbf{3 b}$ 
$(0.061 \mathrm{~g}, 64 \mu \mathrm{L}, 0.50 \mathrm{mmol})$ in the presence of $\mathrm{KF}(0.070 \mathrm{~g}, 1.20 \mathrm{mmol})$ and [18]-crown-6 $(0.317 \mathrm{~g}, 1.20 \mathrm{mmol})$ in THF $(2.0 \mathrm{~mL})$ at $-10{ }^{\circ} \mathrm{C}$ to $\mathrm{rt}$ for $12 \mathrm{~h}$ followed by flash column chromatography (Pet. ether $/$ EtOAc $=99 / 01$ ) of the crude reaction mixture using silica gel afforded N,N-dimethyl-2-(naphthalen-2-yl(phenoxy)methyl)aniline 4ac as a yellow viscous oil (0.93 g, 53\%).

$\boldsymbol{R}_{\mathbf{f}}($ Pet. ether $/$ EtOAc $=97 / 03): 0.38 ;{ }^{1} \mathbf{H}$ NMR $\left(\mathbf{4 0 0} \mathbf{~ M H z}, \mathbf{C D C l}_{3}\right) \delta$ 7.88-7.80 (m, 4H), $7.61(\mathrm{~d}$, $J=8.5 \mathrm{~Hz}, 1 \mathrm{H}), 7.55(\mathrm{~d}, J=7.7 \mathrm{~Hz}, 1 \mathrm{H}), 7.48-7.46(\mathrm{~m}, 2 \mathrm{H}), 7.34-7.21(\mathrm{~m}, 4 \mathrm{H}), 7.14-7.10(\mathrm{~m}$, 2H), 7.07-7.03 (m, 2H), $6.91(\mathrm{t}, J=7.3 \mathrm{~Hz}, 1 \mathrm{H}), 2.71(\mathrm{~s}, 6 \mathrm{H}) .{ }^{13} \mathbf{C}$ NMR (100 MHz, $\left.\mathbf{C D C l}_{3}\right) \delta$ $158.55,152.79,139.13,135.93,133.34,132.95,129.43,129.36,128.88,128.28,127.74,126.33$, 126.15, 126.06, 125.97, 124.51, 120.77, 120.42, 115.94, 75.94, 45.92. HRMS (ESI) calculated $[\mathrm{M}+\mathrm{H}]^{+}$for $\mathrm{C}_{25} \mathrm{H}_{24} \mathrm{ON}: 354.1852$, found: 354.1844. FTIR $\left(\mathbf{c m}^{-1}\right): 3019,2977,2831,2789,1598$, 1493, 1452, 1216, 1047, 1030, 772, 669.

\section{$N, N$-Dimethyl-2-(phenoxy(thiophen-2-yl)methyl)aniline (4ad)}

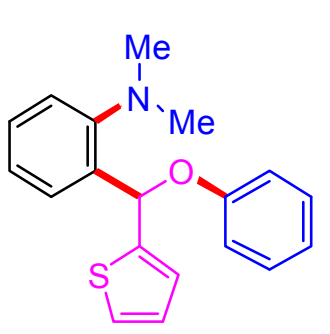

Following the general procedure $\mathbf{A}$, treatment of 2-(trimethylsilyl) phenyltrifluoromethanesulfonate 1a $(0.179 \mathrm{~g}, 146 \mu \mathrm{L}, 0.60 \mathrm{mmol})$ and thiophene-2-carbaldehyde $20(0.112 \mathrm{~g}, 94 \mu \mathrm{L}, 1.0 \mathrm{mmol})$ with $N, N$ dimethylaniline $3 \mathbf{b}(0.061 \mathrm{~g}, 64 \mu \mathrm{L}, 0.50 \mathrm{mmol})$ in the presence of $\mathrm{KF}$ $(0.070 \mathrm{~g}, 1.20 \mathrm{mmol})$ and 18 -crown-6 $(0.317 \mathrm{~g}, 1.20 \mathrm{mmol})$ in THF $(2.0$ $\mathrm{mL}$ ) at $-10{ }^{\circ} \mathrm{C}$ to $\mathrm{rt}$ for $12 \mathrm{~h}$. The reaction mixture was diluted with $\mathrm{CH}_{2} \mathrm{Cl}_{2}(4.0 \mathrm{~mL})$ and filtered through a short pad of silica gel and eluted with $\mathrm{CH}_{2} \mathrm{Cl}_{2}(20.0 \mathrm{~mL})$. The solvent was evaporated to obtain the crude product which was purified by preparative thin-layer chromatography (Pet. ether) to get $N, N$-dimethyl-2-(phenoxy(thiophen-2-yl)methyl)aniline 4ad since the product was unstable in column chromatography. [63\% yield was determined by ${ }^{1} \mathrm{H}$ NMR analysis of crude reaction using $\mathrm{CH}_{2} \mathrm{Br}_{2}(36.0 \mu \mathrm{L}, 0.50 \mathrm{mmol})$ as the internal standard].

$\boldsymbol{R}_{\mathbf{f}}($ Pet. ether $/ \mathrm{EtOAc}=97 / 03): 0.43 ;{ }^{1} \mathbf{H}$ NMR (400 MHz, $\left.\mathbf{C D C l}_{3}\right) \delta 7.74(\mathrm{~d}, J=7.6 \mathrm{~Hz}, 1 \mathrm{H})$, 7.43-7.37 (m, 2H), 7.30-7.26 (m, 3H), 7.21 (t, $J=7.5 \mathrm{~Hz}, 1 \mathrm{H}), 7.12$ (s, 1H), 7.08-7.02 (m, 4H), $6.96(\mathrm{t}, J=7.3 \mathrm{~Hz}, 1 \mathrm{H}), 2.79$ (s, 6H). ${ }^{13} \mathbf{C}$ NMR (100 MHz, $\left.\mathbf{C D C l}_{3}\right) \delta$ 157.93, 152.28, 145.47, 135.48, 129.36, 129.12, 128.53, 126.72, 126.57, 126.14, 124.48, 120.90, 120.04, 115.85, 71.55, 45.88. HRMS (ESI) calculated $[\mathrm{M}+\mathrm{H}]^{+}$for $\mathrm{C}_{19} \mathrm{H}_{20} \mathrm{ONS}: 310.1260$, found: 310.1255 . FTIR ( $\mathbf{c m}^{-}$ 
$\left.{ }^{1}\right)$ : $3066,3015,2934,2862,2786,1930,1592,1490,1450,1299,1224,1088,1038,991,944$, $832,764,702$.

\section{$N, N$-Dimethyl-2-(1-phenoxydecyl)aniline (4ae)}

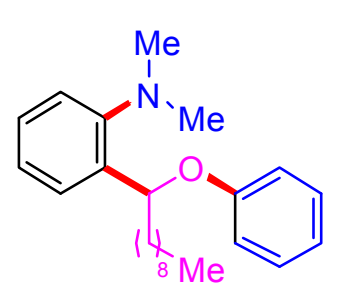

Following the general procedure A, treatment of 2-(trimethylsilyl) phenyltrifluoromethanesulfonate $1 \mathrm{a}(0.179 \mathrm{~g}, 146 \mu \mathrm{L}, 0.60 \mathrm{mmol})$ and decyl aldehyde $2 \mathbf{p}(0.156 \mathrm{~g}, 188 \mu \mathrm{L}, 1.0 \mathrm{mmol})$ with $N, N$-dimethylaniline $\mathbf{3 b}$ $(0.061 \mathrm{~g}, 64 \mu \mathrm{L}, 0.50 \mathrm{mmol})$ in the presence of $\mathrm{KF}(0.070 \mathrm{~g}, 1.20 \mathrm{mmol})$ and 18 -crown-6 $(0.317 \mathrm{~g}, 1.20 \mathrm{mmol})$ in THF $(2.0 \mathrm{~mL})$ at $-10{ }^{\circ} \mathrm{C}$ to $\mathrm{rt}$ for

$12 \mathrm{~h}$ followed by flash column chromatography (Pet. ether) of the crude reaction mixture using silica gel afforded $N, N$-dimethyl-2-(1-phenoxydecyl)aniline 4ae as a colourless viscous oil (0.048 g, 27\% yield).

$\boldsymbol{R}_{\mathbf{f}}($ Pet. ether $/$ EtOAc $=97 / 03): 0.67 ;{ }^{1} \mathbf{H}$ NMR $\left(\mathbf{4 0 0} \mathbf{~ M H z}, \mathbf{C D C l}_{3}\right) \delta 7.40(\mathrm{~d}, J=7.6 \mathrm{~Hz}, 1 \mathrm{H})$, 7.18-7.08 (m, 4H), $7.01(\mathrm{t}, J=7.3 \mathrm{~Hz}, 1 \mathrm{H}), 6.87(\mathrm{~d}, J=8.0 \mathrm{~Hz}, 2 \mathrm{H}), 6.76(\mathrm{t}, J=7.3 \mathrm{~Hz}, 1 \mathrm{H})$, $5.61\left(\mathrm{dd}, J_{1}=9.3 \mathrm{~Hz}, J_{2}=2.8 \mathrm{~Hz}, 1 \mathrm{H}\right), 2.70(\mathrm{~s}, 6 \mathrm{H}), 2.05-1.96(\mathrm{~m}, 1 \mathrm{H}), 1.76-1.57(\mathrm{~m}, 2 \mathrm{H}), 1.49-$ $1.40(\mathrm{~m}, 1 \mathrm{H}), 1.31-1.22(\mathrm{~m}, 12 \mathrm{H}), 0.84(\mathrm{t}, J=6.7 \mathrm{~Hz}, 3 \mathrm{H}) .{ }^{13} \mathbf{C} \mathbf{~ N M R}\left(\mathbf{1 0 0} \mathbf{~ M H z}, \mathbf{C D C l}_{3}\right) \delta$ $158.75,151.79,137.87,129.26,128.22,127.20,124.62,120.23,115.77,74.02,45.95,37.77$, $32.04,29.72,29.58,29.48,26.78,22.82,14.26$. HRMS (ESI) calculated $[\mathrm{M}+\mathrm{H}]^{+}$for $\mathrm{C}_{24} \mathrm{H}_{36} \mathrm{ON}$ : 354.2791, found: 354.2788. FTIR ( $\left.\mathbf{c m}^{-1}\right)$ : 3018, 2929, 2859, 2785, 1593, 1490, 1457, 1297 , $1223,1042,941,767,677$.

\section{2-(Cyclohexyl(phenoxy)methyl)- $N, N$-dimethylaniline (4af)}

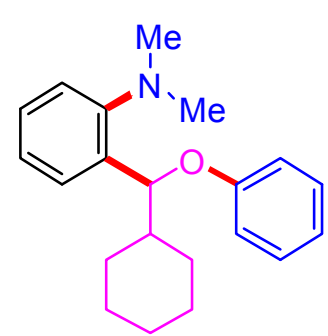

Following the general procedure $\mathbf{A}$, treatment of 2-(trimethylsilyl) phenyltrifluoromethanesulfonate $1 \mathrm{a}(0.179 \mathrm{~g}, 146 \mu \mathrm{L}, 0.60 \mathrm{mmol})$ and cyclohexanecarbaldehyde $2 q(0.112 \mathrm{~g}, 121 \mu \mathrm{L}, 1.0 \mathrm{mmol})$ with $N, N$ dimethylaniline $3 \mathbf{b}(0.061 \mathrm{~g}, 64 \mu \mathrm{L}, 0.50 \mathrm{mmol})$ in the presence of $\mathrm{KF}$

$(0.070 \mathrm{~g}, 1.20 \mathrm{mmol})$ and 18 -crown-6 $(0.317 \mathrm{~g}, 1.20 \mathrm{mmol})$ in THF $(2.0$ $\mathrm{mL}$ ) at $-10{ }^{\circ} \mathrm{C}$ to $\mathrm{rt}$ for $12 \mathrm{~h}$ followed by flash column chromatography (Pet. ether) of the crude reaction mixture using silica gel afforded 2-(cyclohexyl(phenoxy)methyl)- $N, N$-dimethylaniline 4af as a colourless viscous oil ( $0.055 \mathrm{~g}, 35 \%$ yield $)$. 
$\boldsymbol{R}_{\mathbf{f}}($ Pet. ether $/$ EtOAc $=97 / 03): 0.61 ;{ }^{1} \mathbf{H}$ NMR (400 MHz, $\left.\mathbf{C D C l}_{3}\right) \delta 7.50(\mathrm{~d}, J=7.7 \mathrm{~Hz}, 1 \mathrm{H})$, $7.28(\mathrm{~d}, J=3.6 \mathrm{~Hz}, 2 \mathrm{H}), 7.21(\mathrm{t}, J=7.9 \mathrm{~Hz}, 2 \mathrm{H}), 7.17-7.13(\mathrm{~m}, 1 \mathrm{H}), 7.00(\mathrm{~d}, J=8.1 \mathrm{~Hz}, 2 \mathrm{H})$, $6.88(\mathrm{t}, J=7.3 \mathrm{~Hz}, 1 \mathrm{H}), 5.59$ (d, $J=6.2 \mathrm{~Hz}, 1 \mathrm{H}), 2.74$ (s, 6H), 2.09-2.06 (m, 1H), 1.97-1.90 (m, 1H), $1.78-1.73(\mathrm{~m}, 2 \mathrm{H}), 1.54-1.52(\mathrm{~m}, 1 \mathrm{H}), 1.38-1.25(\mathrm{~m}, 6 \mathrm{H}) .{ }^{13} \mathbf{C}$ NMR (100 MHz, CDCl 3$) \delta$ $159.17,152.83,137.12,129.23,128.16,128.00,124.85,121.38,120.44,116.49,79.33,46.40$, 44.37, 30.34, 28.58, 26.76, 26.70, 26.52. HRMS (ESI) calculated $[\mathrm{M}+\mathrm{H}]^{+}$for $\mathrm{C}_{21} \mathrm{H}_{28} \mathrm{ON}$ : 310.2165, found: 310.2164. FTIR (cm $\left.{ }^{-1}\right): 3017,2931,2857,2783,1592,1490,1450,1295$, $1229,1177,988,760$.

\section{4-((2-(Dimethylamino)-4,5-dimethylphenyl)(phenoxy)methyl)benzonitrile (4ag)}

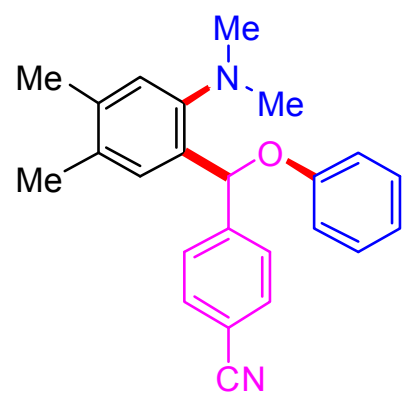

Following the general procedure A, treatment of 4,5-dimethyl-2(trimethylsilyl)phenyl trifluoromethanesulfonate $\mathbf{1 b}(0.196 \mathrm{~g}, 0.60$ mmol) and 4-formylbenzonitrile $2 \mathbf{a}(0.131 \mathrm{~g}, 1.0 \mathrm{mmol})$ with $N, N$ dimethylaniline $3 \mathbf{b}(0.061 \mathrm{~g}, 64 \mu \mathrm{L}, 0.50 \mathrm{mmol})$ in the presence of $\mathrm{KF}(0.070 \mathrm{~g}, 1.20 \mathrm{mmol})$ and 18-crown-6 (0.317 g, $1.20 \mathrm{mmol})$ in THF $(2.0 \mathrm{~mL})$ at $-10{ }^{\circ} \mathrm{C}$ to $\mathrm{rt}$ for $12 \mathrm{~h}$ followed by flash column chromatography $($ Pet. ether/EtOAc $=98 / 02)$ of the crude reaction mixture using silica gel afforded 4-((2-(dimethylamino)-4,5-dimethylphenyl)(phenoxy)methyl)benzonitrile 4ag as a white solid ( $0.154 \mathrm{~g}, 86 \%$ yield).

$\boldsymbol{R}_{\mathbf{f}}($ Pet. ether $/$ EtOAc $=95 / 05): 0.54 ;{ }^{1} \mathbf{H}$ NMR (400 MHz, $\left.\mathbf{C D C l}_{3}\right) \delta 7.62(\mathrm{~d}, J=8.2 \mathrm{~Hz}, 2 \mathrm{H})$, $7.56(\mathrm{~d}, J=8.2 \mathrm{~Hz}, 2 \mathrm{H}), 7.26-7.22(\mathrm{~m}, 2 \mathrm{H}), 7.10$ (s, 1H), 7.04 (s, 1H), 6.99-6.92 (m, 4H), 2.65 (s, 6H), 2.25 (s, 3H), 2.18 (s, 3H). ${ }^{13}$ C NMR (100 MHz, CDCl $) \delta$ 158.07, 150.33, 147.69, $137.85,133.29$, 132.74, 132.28, 129.56, 129.53, 127.94, 122.12, 121.15, 119.04, 115.86, 111.05, 75.13, 46.10, 19.93, 19.50. HRMS (ESI) calculated $[\mathrm{M}+\mathrm{H}]^{+}$for $\mathrm{C}_{24} \mathrm{H}_{25} \mathrm{ON}_{2}: 357.1961$, found: 357.1961. FTIR (cm $\left.{ }^{-1}\right): 3021,2976,2231,1598,1496,1218,1036,768,671$.

\section{X-ray Crystal Structure Analysis of 4 ag}

X-ray intensity data measurements of compound 4ag was carried out on a Bruker SMART APEX II CCD diffractometer with graphite-monochromatized $\left(\mathrm{MoK}_{\alpha}=\right.$ $0.71073 \AA$ ) radiation. The X-ray generator was operated at $50 \mathrm{kV}$ and $30 \mathrm{~mA}$. A preliminary set of cell constants and an orientation matrix were calculated from three sets 
of 36 frames. Data were collected with $\omega$ scan width of $0.5^{\circ}$ at different settings of $\varphi$ and $2 \theta$ with a frame time of 10 secs keeping the sample-to-detector distance fixed at $5.00 \mathrm{~cm}$. The X-ray data collection was monitored by APEX2 program (Bruker, 2006). ${ }^{4}$ All the data were corrected for Lorentzian, polarization and absorption effects using SAINT and SADABS programs (Bruker, 2006). SHELX-97 was used for structure solution and full matrix least-squares refinement on $F^{2}{ }^{5}$ All the hydrogen atoms were placed in geometrically idealized position and constrained to ride on their parent atoms. An ORTEP III $^{6}$ view of both compounds were drawn with $30 \%$ probability displacement ellipsoids and $\mathrm{H}$ atoms are shown as small spheres of arbitrary radii.

Crystal data of $4 \mathbf{a g} \mathrm{C}_{24} \mathrm{H}_{24} \mathrm{~N}_{2} \mathrm{O}, \mathrm{M}=356.45$, colorless block, $0.45 \times 0.23 \times 0.18 \mathrm{~mm}^{3}$, triclinic, space group $P-1, a=$ 10.369(3) $\AA, b=13.940(5) \AA, c$ $=14.494(5) \AA, \alpha=83.533(4)^{\circ}, \beta$ $=85.279(4)^{\circ}, \gamma=75.051(8)^{\circ}, V$ $=2008.0(12) \AA^{3}, \mathrm{Z}=2, T=$ 200(2) $\mathrm{K}, 2 \theta_{\max }=50.00^{\circ}, D_{\text {calc }}(\mathrm{g}$ $\left.\mathrm{cm}^{-3}\right)=1.179, F(000)=760, \mu$ $\left(\mathrm{mm}^{-1}\right)=0.072, \quad 26512$ reflections collected, 6658 unique reflections $\left(R_{\mathrm{int}}=0.0898\right)$, 3694 observed $(I>2 \sigma(I))$

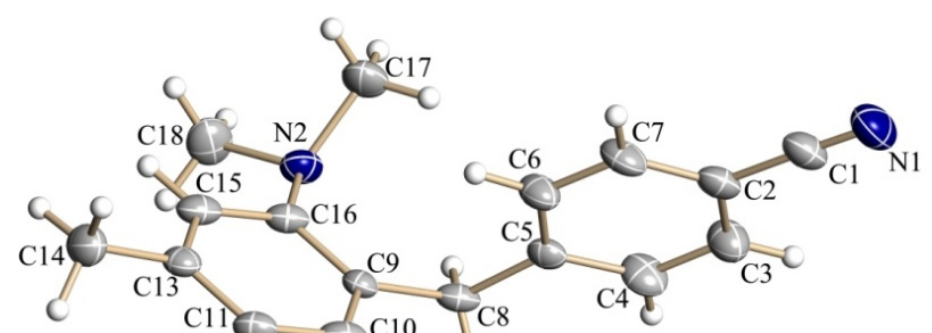
reflections, multi-scan absorption correction, $T_{\min }=0.968, T_{\max }=0.987,334$ refined parameters, $S=1.341, R 1=0.1110, w R 2=0.3108$ (all data $R=0.1847, w R 2=0.4024$ ), maximum and minimum residual electron densities; $\Delta \rho_{\max }=0.43, \Delta \rho_{\min }=-0.41\left(\mathrm{e} \AA^{-3}\right)$.

\footnotetext{
${ }^{4}$ Bruker (2006). APEX2, SAINT and SADABS. Bruker AXS Inc., Madison, Wisconsin, USA.

${ }^{5}$ Sheldrick, G. M. Acta Crystallogr., 2008, A64, 112.

${ }^{6}$ Farrugia, L. J. J. Appl. Cryst. 1997, 30, 565.
} 


\section{4-((6-(Dimethylamino)benzo[d][1,3] dioxol-5-yl)(phenoxy)methyl)benzonitrile (4ah)}

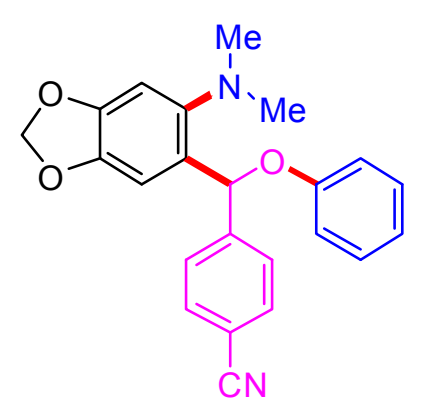

Following the general procedure A, treatment of 6-(trimethylsilyl) benzo $[d][1,3]$ dioxol-5-yl trifluoromethanesulfonate 1c $(0.205 \mathrm{~g}, 0.60$ mmol) and 4-formylbenzonitrile $2 \mathrm{a}(0.131 \mathrm{~g}, 1.0 \mathrm{mmol})$ with $N, N$ dimethylaniline $\mathbf{3 b}(0.061 \mathrm{~g}, 64 \mu \mathrm{L}, 0.50 \mathrm{mmol})$ in the presence of $\mathrm{KF}$ $(0.070 \mathrm{~g}, 1.20 \mathrm{mmol})$ and 18-crown-6 (0.317 $\mathrm{g}, 1.20 \mathrm{mmol})$ in THF $(2.0 \mathrm{~mL})$ at $-10{ }^{\circ} \mathrm{C}$ to $\mathrm{rt}$ for $12 \mathrm{~h}$ followed by flash column chromatography (Pet. ether/EtOAc $=98 / 02$ ) of the crude reaction mixture using silica gel afforded 4-((6-(dimethylamino)benzo[ $d][1,3]$ dioxol-5-yl)(phenoxy)methyl)benzonitrile 4ah as a colourless viscous oil $(0.116 \mathrm{~g}, 62 \%$ yield $)$.

$\boldsymbol{R}_{\mathbf{f}}($ Pet. ether $/$ EtOAc $=80 / 20): 0.66 ;{ }^{1} \mathbf{H}$ NMR (400 MHz, $\left.\mathbf{C D C l}_{3}\right) \delta 7.64(\mathrm{~d}, J=8.4 \mathrm{~Hz}, 2 \mathrm{H})$, $7.55(\mathrm{~d}, J=8.4 \mathrm{~Hz}, 2 \mathrm{H}), 7.28-7.23(\mathrm{~m}, 2 \mathrm{H}), 6.98-6.93(\mathrm{~m}, 4 \mathrm{H}), 6.84-6.82(\mathrm{~m}, 2 \mathrm{H})$, 5.93-5.92 (m, 2H), 2.60 (s, 6H). ${ }^{13} \mathbf{C}$ NMR (100 MHz, $\left.\mathbf{C D C l}_{3}\right) \delta$ 157.82, 148.24, 147.45, 147.13, 145.15, 132.33 , 129.56, 129.12, 127.95, 121.33, 118.95, 115.96, 111.25, 107.56, 102.31, 101.48, 75.30, 46.33. HRMS (ESI) calculated $[\mathrm{M}+\mathrm{H}]^{+}$for $\mathrm{C}_{23} \mathrm{H}_{21} \mathrm{O}_{3} \mathrm{~N}_{2}: 373.1547$, found: 373.1542 . FTIR (cm ${ }^{-}$ 1): 3021, 2896, 2784, 2231, 1597, 1490, 1223, 1040, 764.

\section{4-((2-(Dimethylamino)-4,5-difluorophenyl)(phenoxy)methyl)benzonitrile (4ai)}

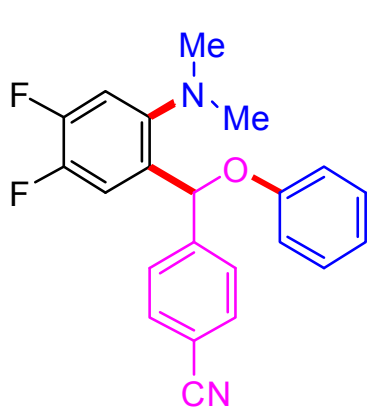

Following the general procedure A, treatment of 4,5-difluoro-2(trimethylsilyl)phenyl trifluoromethanesulfonate 1d (0.201 g, 0.60 mmol) and 4-formylbenzonitrile $2 \mathrm{a}(0.131 \mathrm{~g}, 1.0 \mathrm{mmol})$ with $N, N$ dimethylaniline $\mathbf{3 b}(0.061 \mathrm{~g}, 64 \mu \mathrm{L}, 0.50 \mathrm{mmol})$ in the presence of $\mathrm{KF}$ $(0.070 \mathrm{~g}, 1.20 \mathrm{mmol})$ and 18-crown-6 (0.317 $\mathrm{g}, 1.20 \mathrm{mmol})$ in THF $(2.0 \mathrm{~mL})$ at $-10{ }^{\circ} \mathrm{C}$ to $\mathrm{rt}$ for $12 \mathrm{~h}$ followed by flash column chromatography (Pet. ether/EtOAc $=98 / 02)$ of the crude reaction mixture using silica gel afforded 4-((2-(dimethylamino)-4,5-difluorophenyl)(phenoxy)methyl)benzonitrile 4ai as a colourless viscous oil ( $0.073 \mathrm{~g}, 40 \%$ yield).

$\boldsymbol{R}_{\mathbf{f}}\left(\right.$ Pet. ether $/$ EtOAc $=$ 95/05): 0.44; ${ }^{\mathbf{H}} \mathbf{H}$ NMR (400 $\left.\mathbf{M H z}, \mathbf{C D C l}_{3}\right) \delta 7.64(\mathrm{~d}, J=8.1 \mathrm{~Hz}, 2 \mathrm{H})$, $7.53(\mathrm{~d}, J=8.1 \mathrm{~Hz}, 2 \mathrm{H}), 7.27-7.18(\mathrm{~m}, 3 \mathrm{H}), 7.08-7.04(\mathrm{~m}, 1 \mathrm{H}), 6.97-6.92(\mathrm{~m}, 3 \mathrm{H}), 6.87(\mathrm{~s}, 1 \mathrm{H})$, $2.62(\mathrm{~s}, 6 \mathrm{H}) .{ }^{13} \mathbf{C}$ NMR (100 MHz, $\left.\mathbf{C D C l}_{3}\right) \delta 157.45,151.40\left(\mathrm{dd}, J_{1}=251.6 \mathrm{~Hz}, J_{2}=13.6 \mathrm{~Hz}\right)$, $147.71\left(\mathrm{dd}, J_{l}=246.5 \mathrm{~Hz}, J_{2}=13.0 \mathrm{~Hz}\right), 146.37,132.50,129.70,127.94,121.73,118.74,117.09$ 
(d, $J=18.4 \mathrm{~Hz}), 115.85,111.74,110.44(\mathrm{~d}, J=16.7 \mathrm{~Hz}), 74.67,46.01$. HRMS (ESI) calculated $[\mathrm{M}+\mathrm{H}]^{+}$for $\mathrm{C}_{22} \mathrm{H}_{19} \mathrm{ON}_{2} \mathrm{~F}_{2}: 365.1460$, found: 365.1454. FTIR $\left(\mathbf{c m}^{-1}\right): 3022,2789,2404,2232$, $1599,1506,1219,766,673$.

\section{4-((3-(dimethylamino)naphthalen-2-yl)(phenoxy)methyl)benzonitrile (4aj)}

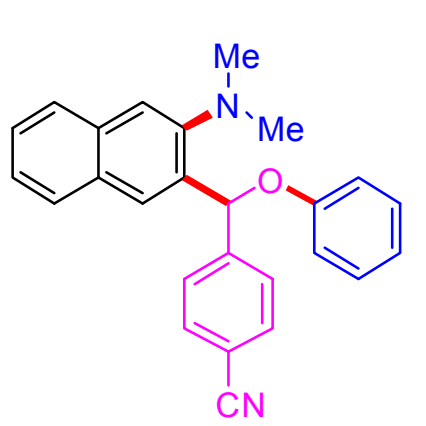

Following the general procedure $\mathbf{A}$, treatment of 3(trimethylsilyl)naphthalen-2-yl trifluoromethanesulfonate 1e $(0.210 \mathrm{~g}$, $0.60 \mathrm{mmol})$ and 4-formylbenzonitrile $2 \mathrm{a}(0.131 \mathrm{~g}, 1.0 \mathrm{mmol})$ with $N, N$-dimethylaniline $3 \mathbf{b}(0.061 \mathrm{~g}, 64 \mu \mathrm{L}, 0.50 \mathrm{mmol})$ in the presence of $\mathrm{KF}(0.070 \mathrm{~g}, 1.20 \mathrm{mmol})$ and [18]-crown-6 (0.317 g, $1.2 \mathrm{mmol})$ in THF $(2.0 \mathrm{~mL})$ at $-10{ }^{\circ} \mathrm{C}$ to $\mathrm{rt}$ for $12 \mathrm{~h}$ followed by flash column chromatography (Pet. ether $/$ EtOAc $=98 / 02$ ) of the crude reaction mixture using silica gel afforded 4-((3-(dimethylamino)naphthalen-2-yl)(phenoxy)methyl)benzonitrile 4aj as a colourless viscous oil ( $0.096 \mathrm{~g}, 48 \%)$.

$\boldsymbol{R}_{\mathbf{f}}(\mathrm{Pet}$. ether $/ \mathrm{EtOAc}=95 / 05): 0.46 ;{ }^{1} \mathbf{H}$ NMR (400 MHz, $\left.\mathbf{C D C l}_{\mathbf{3}}\right) \delta$ 7.92, (s, $\left.1 \mathrm{H}\right), 7.78(\mathrm{t}, J=$ 8.2 Hz, 2H), 7.66-7.61 (m, 5H), 7.47-7.45 (m, 1H), 7.42-7.38 (m, 1H), 7.30-7.26 (m, 2H), 7.05$7.02(\mathrm{~m}, 3 \mathrm{H}), 6.97(\mathrm{t}, J=7.3 \mathrm{~Hz}, 1 \mathrm{H}), 2.79(\mathrm{~s}, 6 \mathrm{H}) .{ }^{13} \mathbf{C} \mathbf{~ N M R}\left(\mathbf{1 0 0} \mathbf{~ M H z}, \mathbf{C D C l}_{\mathbf{3}}\right) \delta$ 158.00, $150.71,147.29,135.31,133.93,132.26,131.18,130.76,129.61,128.66,128.19,128.03,126.89$, 126.63, 125.23, 121.40, 118.90, 118.26, 115.92, 111.31, 77.48, 77.16, 76.84, 76.03, 45.93. HRMS (ESI) calculated $[\mathrm{M}+\mathrm{H}]^{+}$for $\mathrm{C}_{26} \mathrm{H}_{23} \mathrm{ON}_{2}: 379.1805$, found: 379.1802 . FTIR (cm $\left.{ }^{-1}\right)$ : 3020, 2941, 2788, 2230, 1595, 1492, 1221, 1022, 760, 673.

\section{4-((2-(Dimethylamino)naphthalen-1-yl)(phenoxy)methyl)benzonitrile (4ak)}

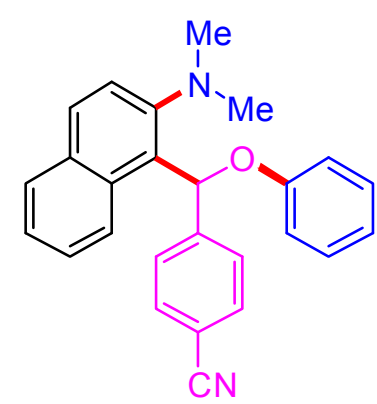

mixture using silica gel afforded 4-((2-(dimethylamino)naphthalen-1-yl)(phenoxy)methyl) (trimethylsilyl)naphthalen-2-yl trifluoromethanesulfonate $\mathbf{1 f}(0.209 \mathrm{~g}$, $0.60 \mathrm{mmol})$ and 4-formylbenzonitrile $2 \mathrm{a}(0.131 \mathrm{~g}, 1.0 \mathrm{mmol})$ with $N, N$ dimethylaniline $3 \mathbf{b}(0.061 \mathrm{~g}, 64 \mu \mathrm{L}, 0.50 \mathrm{mmol})$ in the presence of $\mathrm{KF}$ $(0.070 \mathrm{~g}, 1.20 \mathrm{mmol})$ and [18]-crown-6 (0.317 g, $1.20 \mathrm{mmol})$ in THF $(2.0 \mathrm{~mL})$ at $-10{ }^{\circ} \mathrm{C}$ to $\mathrm{rt}$ for $12 \mathrm{~h}$ followed by flash column chromatography (Pet. ether $/$ EtOAc $=98 / 02)$ of the crude reaction

Following the general procedure $\mathbf{A}$, treatment of 1- 
benzonitrile 4ak as a yellow viscous oil $(0.050 \mathrm{~g}, 26 \%)$ and $N$-methyl- $N$-phenylnaphthalen-2amine $^{3}$ as a colorless oil $(0.047 \mathrm{~g}, 41 \%) .{ }^{3} \mathrm{H}-\mathrm{NMR}$ analysis of crude reaction mixture shows formation of single regioisomer, no decteable amount of $\mathbf{4 a k}$ '.

$\boldsymbol{R}_{\mathbf{f}}($ Pet. ether $/$ EtOAc $=95 / 05): 0.46 ;{ }^{1} \mathbf{H}$ NMR $\left(400 \mathbf{~ M H z}, \mathbf{C D C l}_{3}\right) \delta 8.02(\mathrm{~d}, J=8.4 \mathrm{~Hz}, 1 \mathrm{H})$, $7.88(\mathrm{~d}, J=8.8 \mathrm{~Hz}, 1 \mathrm{H}), 7.77(\mathrm{~d}, J=7.7 \mathrm{~Hz}, 1 \mathrm{H}), 7.71 \quad(\mathrm{~s}, 1 \mathrm{H}), 7.61-7.59(\mathrm{~m}, 2 \mathrm{H}), 7.53-7.47$ (m, 3H), 7.37-7.27 (m, 2H), 7.23-7.19 (m, 2H), 7.12-7.10 (m, 2H), 6.90 (t, $J=7.3 \mathrm{~Hz}, 1 \mathrm{H}), 2.78$ (s, 6H). ${ }^{13} \mathbf{C}$ NMR (100 MHz, $\left.\mathbf{C D C l}_{3}\right) \delta 158.42,151.54,147.77,132.22,131.92,131.05,129.43$, 128.48, 127.84, 127.63, 127.10, 126.17, 124.90, 121.36, 119.37, 119.04, 116.08, 110.97, 75.03, 46.13. HRMS (ESI) calculated $[\mathrm{M}+\mathrm{H}]^{+}$for $\mathrm{C}_{26} \mathrm{H}_{23} \mathrm{ON}_{2}: 379.1805$, found: 379.1805 . FTIR (cm ${ }^{-}$ 1): 3019, 2937, 2866, 2837, 2789, 2230, 1596, 1495, 1301, 1224, 1016, 992, 820, 760, 680.

\section{4-((2-(Dimethylamino)-5-methylphenyl)(phenoxy)methyl)benzonitrile (4al) and 4-((2-(Dimethylamino)-4-methylphenyl)(phenoxy)methyl)benzonitrile (4al')}

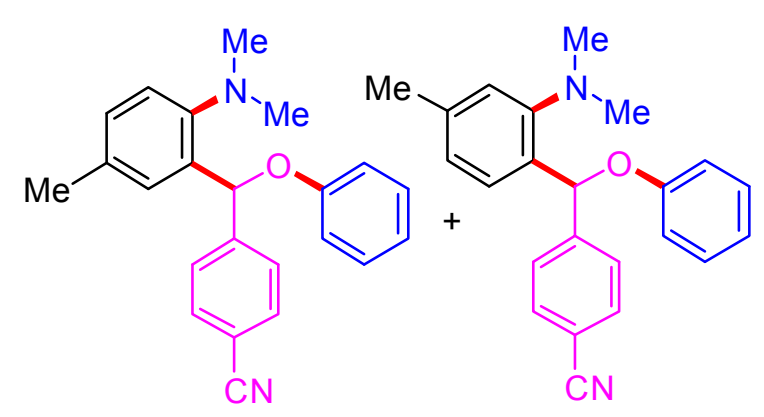

Following the general procedure $\mathbf{A}$, treatment of 5-methyl-2-(trimethylsilyl)phenyltrifluoromethan esulfonate $1 \mathrm{~g}(0.187 \mathrm{~g}, \quad 0.60 \mathrm{mmol})$ and $4-$ formylbenzonitrile $2 \mathrm{a}(0.131 \mathrm{~g}, 1.0 \mathrm{mmol})$ with $N, N$-dimethylaniline 3b $(0.061 \mathrm{~g}, 64 \mu \mathrm{L}, 0.50$ $\mathrm{mmol})$ in the presence of $\mathrm{KF}(0.070 \mathrm{~g}, 1.20$ mmol) and [18]-crown-6 $(0.317 \mathrm{~g}, 1.20 \mathrm{mmol})$ in THF $(2.0 \mathrm{~mL})$ at $-10{ }^{\circ} \mathrm{C}$ to $\mathrm{rt}$ for $12 \mathrm{~h}$ followed by flash column chromatography (Pet. ether $/ \mathrm{EtOAc}=98 / 02$ ) of the crude reaction mixture using silica gel afforded 4-((2-(dimethylamino)-5 methylphenyl)(phenoxy)methyl) benzonitrile 4al and 4-((2-(dimethylamino)-4 methylphenyl)(phenoxy)methyl)benzonitrile 4al' as a mixture of regioisomers in 1:1 ratio $(0.105 \mathrm{~g}, 61 \%$ yield, colourless viscous oil, regioisomer ratio determined by ${ }^{1} \mathrm{H}-\mathrm{NMR}$ analysis of crude reaction mixture ).

$\boldsymbol{R}_{\mathbf{f}}($ Pet. ether $/$ EtOAc $=95 / 05): 0.52 ;{ }^{1} \mathbf{H}$ NMR (400 MHz, $\left.\mathbf{C D C l}_{3}\right) \delta 7.63(\mathrm{~d}, J=8.2 \mathrm{~Hz}, 2 \mathrm{H})$, $7.57(\mathrm{~d}, J=8.2 \mathrm{~Hz}, 2 \mathrm{H}), 7.28-7.23(\mathrm{~m}, 2 \mathrm{H}), 7.21(\mathrm{~s}, 1 \mathrm{H}), 7.15-7.13(\mathrm{~m}, 1 \mathrm{H}), 7.07(\mathrm{~s}, 1 \mathrm{H}), 7.00-$ $6.92(\mathrm{~m}, 4 \mathrm{H}), 2.68(\mathrm{~s}, 6 \mathrm{H}), 2.35(\mathrm{~s}, 3 \mathrm{H})$. Representative peaks of other isomer ${ }^{1} \mathbf{H}$ NMR (400 MHz, $\left.\mathbf{C D C l}_{3}\right) \delta 7.63(\mathrm{~d}, J=8.3 \mathrm{~Hz}, 2 \mathrm{H}), 7.58(\mathrm{~d}, J=8.32 \mathrm{~Hz}, 2 \mathrm{H}), 7.18(\mathrm{~s}, 1 \mathrm{H}), 2.66(\mathrm{~s}, 6 \mathrm{H})$, 2.29 (s, 3H). ${ }^{13} \mathbf{C}$ NMR (100 MHz, $\left.\mathbf{C D C l}_{3}\right) \delta 157.99,152.55,147.55,139.26,134.59,132.25$, $130.07,129.53,128.67,128.05,125.71,121.52,118.95,115.85,111.16,75.28,45.87,21.43$. 
Representative peaks of other isomer ${ }^{13} \mathbf{C}$ NMR (100 $\left.\mathbf{~ M H z}, \mathbf{C D C l}_{3}\right) \delta 158.02,150.10,147.49$, 135.38, 128.97, 132.34, 129.54, 127.99, 121.25, 121.19, 120.95, 115.90, 75.37, 46.06, 21.11. HRMS (ESI) calculated $[\mathrm{M}+\mathrm{H}]{ }^{+}$for $\mathrm{C}_{23} \mathrm{H}_{23} \mathrm{ON}_{2}: 343.1805$, found: 343.1804 . FTIR $\left(\mathbf{c m}^{-1}\right)$ : $3021,2937,2865,2832,2784,2229,1597,1494,1298,1227,1173,1022,827,759$.

4-((2-(Dimethylamino)-5-fluorophenyl)(phenoxy)methyl)benzonitrile (4am) and 4-((2-(Dimethylamino)-4-fluorophenyl)(phenoxy)methyl)benzonitrile (4am')

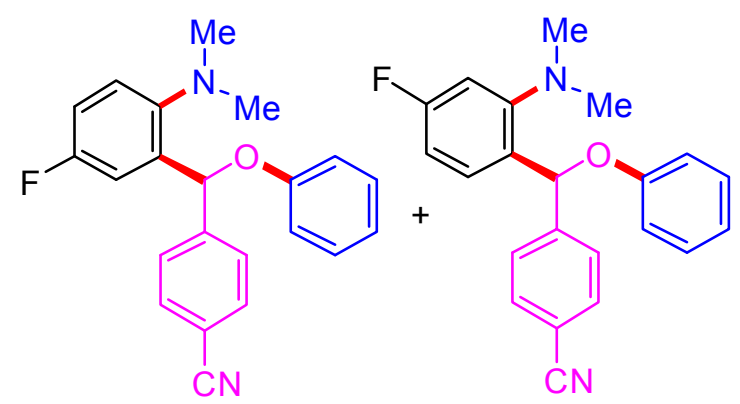

Following the general procedure $\mathbf{A}$, treatment of 5fluoro-2-(trimethylsilyl)phenyl trifluoromethane sulfonate $\mathbf{1 h}(0.189 \mathrm{~g}, \quad 0.60 \mathrm{mmol})$ and $4-$ formylbenzonitrile $2 \mathrm{a}(0.131 \mathrm{~g}, 1.0 \mathrm{mmol})$ with $N, N$-dimethylaniline 3b $(0.061 \mathrm{~g}, 64 \mu \mathrm{L}, 0.50$ $\mathrm{mmol})$ in the presence of $\mathrm{KF}(0.070 \mathrm{~g}, 1.2 \mathrm{mmol})$ and [18]-crown-6 $(0.317 \mathrm{~g}, 1.20 \mathrm{mmol})$ in THF $(2.0 \mathrm{~mL})$ at $-10{ }^{\circ} \mathrm{C}$ to $\mathrm{rt}$ for $12 \mathrm{~h}$ followed by flash column chromatography (Pet. ether $/ \mathrm{EtOAc}=98 / 02)$ of the crude reaction mixture using silica gel afforded 4-((2-(dimethylamino)-5-fluorophenyl)(phenoxy)methyl)benzonitrile 4am and 4-((2-(dimethylamino)-4-fluorophenyl)(phenoxy)methyl)benzonitrile 4am' as a mixture of regioisomers in 2.2:1 ratio $(0.118 \mathrm{~g}, 68 \%$ yield, colorless viscous oil, regioisomer ratio determined by ${ }^{1} \mathrm{H}-\mathrm{NMR}$ analysis of crude reaction mixture).

$\boldsymbol{R}_{\mathbf{f}}($ Pet. ether $/$ EtOAc $=95 / 05): 0.48 ;{ }^{1} \mathbf{H}$ NMR $\left(400 \mathbf{~ M H z}, \mathbf{C D C l}_{3}\right) \delta$ 7.64-7.61 (m, 2H), 7.56$7.50(\mathrm{~m}, 2 \mathrm{H}), 7.32-7.21(\mathrm{~m}, 3 \mathrm{H}), 7.13\left(\mathrm{dd}, J_{1}=9.4, J_{2}=3.0 \mathrm{~Hz}, 1 \mathrm{H}\right) 7.01-6.90(\mathrm{~m}, 4 \mathrm{H}), 6.83-$ $6.77(\mathrm{~m}, 1 \mathrm{H}), 2.60(\mathrm{~s}, 6 \mathrm{H})$. Representative peaks of other isomer ${ }^{1} \mathrm{H}$ NMR $\left(400 \mathrm{MHz}, \mathbf{C D C l}_{3}\right)$ $\delta 2.66(\mathrm{~s}) .{ }^{13} \mathbf{C}$ NMR (100 MHz, $\left.\mathbf{C D C l}_{3}\right) \delta 160.10(\mathrm{~d}, J=244.4 \mathrm{~Hz}), 157.66,148.42(\mathrm{~d}, J=2.5$ $\mathrm{Hz}), 146.67,138.17$ (d, $J=7.0 \mathrm{~Hz}), 128.06,123.07$ (d, $J=8.3 \mathrm{~Hz}), 121.58,116.22,116.00$, 115.93, $114.87(\mathrm{~d}, J=23.2 \mathrm{~Hz}), 111.59,75.26,46.17$. Representative peaks of other isomer ${ }^{13}$ C NMR (100 MHz, CDCl $) \delta 163.24(\mathrm{~d}, J=248.4 \mathrm{~Hz}), 157.76,154.64(\mathrm{~d}, J=7.8 \mathrm{~Hz})$, 147.05, 132.40, 130.68 (d, $J=9.6 \mathrm{~Hz}), 129.64,128.03,121.45,118.87,115.78,111.65$ (d, $J=$ $21.7 \mathrm{~Hz}), 111.47,107.91(\mathrm{~d}, J=21.5 \mathrm{~Hz}), 74.94,45.67$. HRMS (ESI) calculated $[\mathrm{M}+\mathrm{H}]^{+}$for $\mathrm{C}_{22} \mathrm{H}_{20} \mathrm{FON}_{2}: 347.1554$, found: 347.1552. FTIR $\left(\mathrm{cm}^{-1}\right)$ : 3021, 2944, 2839, 2789, 2231, 1598, 1493, 1300, 1223, 1022, 993, 761, 679. 


\section{3-(2-(Dimethylamino)phenyl)-1-methyl-3-phenoxyindolin-2-one (6a)}

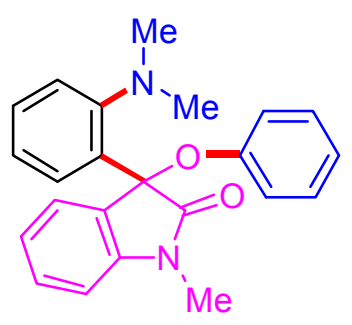

Following the general procedure, treatment of 2-(trimethylsilyl) phenyltrifluoromethanesulfonate $1 \mathbf{a}(0.179 \mathrm{~g}, 146 \mu \mathrm{L}, 0.60 \mathrm{mmol})$ and 1-

methylindoline-2,3-dione 5a $(0.161 \mathrm{~g}, 1.0 \mathrm{mmol})$ with $N, N-$ dimethylaniline $3 \mathbf{b}(0.061 \mathrm{~g}, 64 \mu \mathrm{L}, 0.50 \mathrm{mmol})$ in the presence of $\mathrm{KF}$ $(0.070 \mathrm{~g}, 1.20 \mathrm{mmol})$ and 18-crown-6 (0.317 $\mathrm{g}, 1.20 \mathrm{mmol})$ in THF $(2.0$ $\mathrm{mL}$ ) at $-10{ }^{\circ} \mathrm{C}$ to $\mathrm{rt}$ for $12 \mathrm{~h}$ followed by flash column chromatography (Pet. ether/EtOAc $=$ 90/10) of the crude reaction mixture using silica gel afforded 3-(2-(dimethylamino)phenyl)-1methyl-3-phenoxyindolin-2-one 6a as a white solid (0.128 g, 72\% yield).

$\boldsymbol{R}_{\mathbf{f}}($ Pet. ether $/$ EtOAc $=70 / 30)$ : 0.63; ${ }^{1} \mathbf{H}$ NMR (400 $\left.\mathbf{M H z}, \mathbf{C D C l}_{3}\right) \delta$ 8.20-8.18 (m, 1H), 7.36$7.29(\mathrm{~m}, 2 \mathrm{H}), 7.24-7.20(\mathrm{~m}, 1 \mathrm{H}), 7.14(\mathrm{t}, J=7.7 \mathrm{~Hz}, 1 \mathrm{H}), 7.07-7.01(\mathrm{~m}, 3 \mathrm{H}), 6.93-6.84(\mathrm{~m}, 4 \mathrm{H})$, $6.57(\mathrm{~d}, J=7.8 \mathrm{~Hz}, 1 \mathrm{H}), 3.03$ (s, 3H), 2.39 (bs, 3H), 1.62 (bs, 3H). ${ }^{13} \mathbf{C}$ NMR (100 MHz, $\left.\mathbf{C D C l}_{3}\right) \delta 174.35,154.56,150.93,144.98,138.46,129.72,129.08,128.98,128.48,127.40$, 126.47, 125.34, 124.16, 123.53, 123.39, 121.93, 107.69, 84.22, 45.32, 44.51, 25.93. HRMS (ESI) calculated $[\mathrm{M}+\mathrm{H}]^{+}$for $\mathrm{C}_{23} \mathrm{H}_{23} \mathrm{O}_{2} \mathrm{~N}_{2}: 359.1754$, found: 359.1755 . FTIR $\left(\mathbf{c m}^{-1}\right): 3020,1720$, $1608,1484,1217,926,768,670$.

\section{5-Bromo-3-(2-(dimethylamino)phenyl)-1-methyl-3-phenoxyindolin-2-one (6b)}

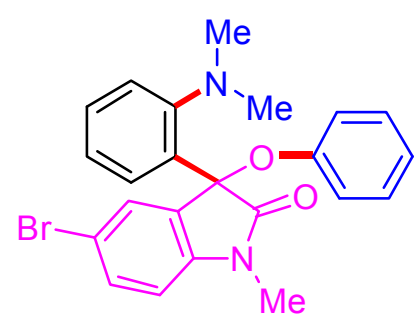

Following the general procedure, treatment of 2-(trimethylsilyl) phenyltrifluoromethanesulfonate 1a $(0.179 \mathrm{~g}, 146 \mu \mathrm{L}, 0.60 \mathrm{mmol})$ and 5-bromo-1-methylindoline-2,3-dione $5 \mathbf{b}(0.240 \mathrm{~g}, 1.0 \mathrm{mmol})$ with $N, N$-dimethylaniline 3b $(0.061 \mathrm{~g}, 64 \mu \mathrm{L}, 0.50 \mathrm{mmol})$ in the presence of $\mathrm{KF}(0.070 \mathrm{~g}, 1.20 \mathrm{mmol})$ and 18-crown-6 (0.317 g, $1.20 \mathrm{mmol})$ in THF $(2.0 \mathrm{~mL})$ at $-10{ }^{\circ} \mathrm{C}$ to $\mathrm{rt}$ for $12 \mathrm{~h}$ followed by flash column chromatography (Pet. ether/EtOAc $=90 / 10)$ of the crude reaction mixture using silica gel afforded 5-bromo-3-(2(dimethylamino)phenyl)-1-methyl-3-phenoxyindolin-2-one $6 \mathbf{b}$ as a white solid (0.160 g, 73\% yield).

$\boldsymbol{R}_{\mathbf{f}}($ Pet. ether $/$ EtOAc $=70 / 30)$ : 0.60; ${ }^{1} \mathbf{H}$ NMR (400 MHz, $\left.\mathbf{C D C l}_{3}\right) \delta 8.22-8.18(\mathrm{~m}, 1 \mathrm{H}), 7.42-$ $7.38(\mathrm{~m}, 2 \mathrm{H}), 7.33\left(\mathrm{dd}, J_{1}=8.3 \mathrm{~Hz}, J_{2}=2.0 \mathrm{~Hz}, 1 \mathrm{H}\right), 7.29-7.27(\mathrm{~m}, 1 \mathrm{H}), 7.24(\mathrm{~d}, J=1.9 \mathrm{~Hz}$, 1H), 7.13-7.10 (m, 2H), $7.00(\mathrm{t}, J=7.3 \mathrm{~Hz}, 1 \mathrm{H}), 6.89(\mathrm{~d}, J=7.7 \mathrm{~Hz}, 2 \mathrm{H}), 6.51(\mathrm{~d}, J=8.3 \mathrm{~Hz}$, 1H), 3.05 (s, 3H), 2.42 (bs, 3H), 1.73 (bs, 3H). ${ }^{13}$ C NMR (100 MHz, CDCl $\mathbf{3}$ ) $\delta 173.71,154.32$, 
$150.90,144.04,137.77,132.48,131.37,129.37,128.69,128.25,127.43,126.74,124.48,123.69$, 123.32, 114.36, 109.23, 83.93, 45.61, 44.58, 26.04. HRMS (ESI) calculated $[\mathrm{M}+\mathrm{H}]^{+}$for $\mathrm{C}_{23} \mathrm{H}_{22} \mathrm{O}_{2} \mathrm{~N}_{2} \mathrm{Br}: 437.0859$, found: 437.0852. FTIR $\left(\mathbf{c m}^{-1}\right)$ : 3018, 2938, 2868, 2830, 2786, 1725 , 1602, 1484, 1346, 1216, 1100, 769, 701.

\section{3-(2-(Dimethylamino)phenyl)-5-fluoro-1-methyl-3-phenoxyindolin-2-one (6c)}

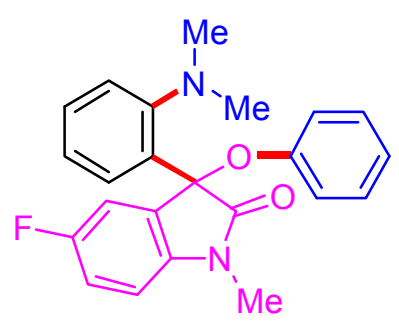

Following the general procedure, treatment of 2-(trimethylsilyl) phenyltrifluoromethanesulfonate $1 \mathrm{a}(0.179 \mathrm{~g}, 146 \mu \mathrm{L}, 0.60 \mathrm{mmol})$ and 5-fluoro-1-methylindoline-2,3-dione $\mathbf{5 c}(0.179 \mathrm{~g}, 1.0 \mathrm{mmol})$ with $N, N$ dimethylaniline $\mathbf{3 b}(0.061 \mathrm{~g}, 64 \mu \mathrm{L}, 0.50 \mathrm{mmol})$ in the presence of $\mathrm{KF}$ $(0.070 \mathrm{~g}, 1.20 \mathrm{mmol})$ and 18 -crown-6 $(0.317 \mathrm{~g}, 1.20 \mathrm{mmol})$ in THF $(2.0 \mathrm{~mL})$ at $-10{ }^{\circ} \mathrm{C}$ to $\mathrm{rt}$ for $12 \mathrm{~h}$ followed by flash column chromatography (Pet. ether/EtOAc $=$ 90/10) of the crude reaction mixture using silica gel afforded 3-(2-(dimethylamino)phenyl)-5fluoro-1-methyl-3-phenoxyindolin-2-one $\mathbf{6 c}$ as a white solid $(0.139 \mathrm{~g}, 74 \%$ yield).

$\boldsymbol{R}_{\mathbf{f}}($ Pet. ether $/$ EtOAc $=70 / 30): 0.58 ;{ }^{1} \mathbf{H}$ NMR $\left(\mathbf{4 0 0} \mathbf{~ M H z}, \mathbf{C D C l}_{\mathbf{3}}\right) \delta 8.22-8.20(\mathrm{~m}, 1 \mathrm{H}), 7.43-$ $7.37(\mathrm{~m}, 2 \mathrm{H}), 7.29-7.27(\mathrm{~m}, 1 \mathrm{H}), 7.14-7.10(\mathrm{~m}, 2 \mathrm{H}), 7.01(\mathrm{t}, J=7.3 \mathrm{~Hz}, 1 \mathrm{H}), 6.94-6.87(\mathrm{~m}, 4 \mathrm{H})$, $6.56\left(\mathrm{dd}, J_{1}=8.4 \mathrm{~Hz}, J_{2}=4.0 \mathrm{~Hz}, 1 \mathrm{H}\right), 3.08(\mathrm{~s}, 3 \mathrm{H}), 2.44(\mathrm{bs}, 3 \mathrm{H}), 1.72(\mathrm{bs}, 3 \mathrm{H}) .{ }^{13} \mathbf{C}$ NMR (100 MHz, $\left.\mathbf{C D C l}_{3}\right) \delta 174.25,158.87(\mathrm{~d}, J=240.9 \mathrm{~Hz}), 154.38,150.98,141.10,137.89,130.76(\mathrm{~d}, J$ $=7.2 \mathrm{~Hz}), 129.38,128.72,127.44,126.78,124.52,123.72,123.45,115.95$ (d, $J=23.5 \mathrm{~Hz})$, $113.25(\mathrm{~d}, J=24.5 \mathrm{~Hz}), 108.24(\mathrm{~d}, J=7.7 \mathrm{~Hz}), 84.24,45.58,44.63,26.18$. HRMS (ESI) calculated $[\mathrm{M}+\mathrm{H}]^{+}$for $\mathrm{C}_{23} \mathrm{H}_{22} \mathrm{O}_{2} \mathrm{~N}_{2} \mathrm{~F}$ : 377.1660, found: 377.1659. FTIR $\left(\mathbf{c m}^{-1}\right)$ : 3017, 2938, 2868, 2829, 2785, 1722, 1599, 1489, 1460, 1266, 1214, 1132, 879, 767, 673.

\section{3-(2-(Dimethylamino)phenyl)-1-methyl-3-(p-tolyloxy)indolin-2-one (6d)}

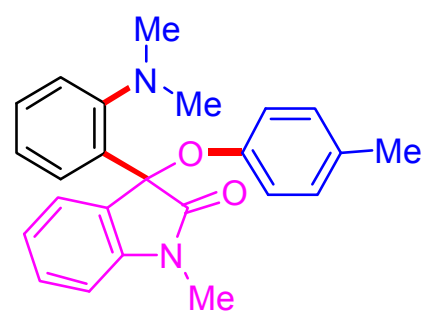

Following the general procedure, treatment of 2-(trimethylsilyl) phenyltrifluoromethanesulfonate 1a $(0.179 \mathrm{~g}, 146 \mu \mathrm{L}, 0.60 \mathrm{mmol})$ and 1-methylindoline-2,3-dione $5 \mathbf{a}(0.161 \mathrm{~g}, 1.0 \mathrm{mmol})$ with $N, N, 4-$ trimethylaniline $3 \mathbf{c}(0.068 \mathrm{~g}, 73 \mu \mathrm{L}, 0.50 \mathrm{mmol})$ in the presence of $\mathrm{KF}(0.070 \mathrm{~g}, 1.20 \mathrm{mmol})$ and 18-crown-6 $(0.317 \mathrm{~g}, 1.20 \mathrm{mmol})$ in

THF $(2.0 \mathrm{~mL})$ at $-10{ }^{\circ} \mathrm{C}$ to $\mathrm{rt}$ for $12 \mathrm{~h}$ followed by flash column chromatography (Pet.ether/EtOAc $=90 / 10)$ of the crude reaction mixture using silica gel afforded 3-(2- 
(dimethylamino)phenyl)-1-methyl-3-(p-tolyloxy)indolin-2-one $6 \mathbf{d}$ as a white solid (0.136 g, $73 \%$ yield).

$\boldsymbol{R}_{\mathbf{f}}($ Pet. ether $/$ EtOAc $=70 / 30)$ : 0.65; ${ }^{1} \mathbf{H}$ NMR (400 $\left.\mathbf{M H z}, \mathbf{C D C l}_{3}\right) \delta$ 8.23-8.19 (m, 1H), 7.39$7.32(\mathrm{~m}, 2 \mathrm{H}), 7.25-7.22(\mathrm{~m}, 1 \mathrm{H}), 7.18(\mathrm{t}, J=7.7 \mathrm{~Hz}, 1 \mathrm{H}), 7.08(\mathrm{~d}, J=7.0 \mathrm{~Hz}, 1 \mathrm{H}), 6.91(\mathrm{t}, J=$ $7.5 \mathrm{~Hz}, 1 \mathrm{H}), 6.85(\mathrm{~d}, J=8.4 \mathrm{~Hz}, 2 \mathrm{H}), 6.74(\mathrm{~d}, J=8.4 \mathrm{~Hz}, 2 \mathrm{H}), 6.60$ (d, $J=7.8 \mathrm{~Hz}, 1 \mathrm{H}), 3.07$ (s, 3H), 2.41 (bs, 3H), 2.18 (s, 3H), 1.62 (bs, 3H). ${ }^{13} \mathbf{C}$ NMR (100 MHz, CDCl $\mathbf{~}_{3} \delta$ 174.65, 152.29, $150.98,145.12$, 138.64, 133.60, 129.68, 129.28, 129.09, 129.01, 127.52, 126.57, 125.46, 123.56, 123.23, 121.96, 107.74, 84.19, 45.50, 44.63, 26.05, 20.84. HRMS (ESI) calculated $[\mathrm{M}+\mathrm{H}]^{+}$for $\mathrm{C}_{24} \mathrm{H}_{25} \mathrm{O}_{2} \mathrm{~N}_{2}$ : 373.1911, found: 373.1906. FTIR (cm $\left.{ }^{-1}\right): 3021,2785,2354,1720,1612,1499$, $1217,929,769,671$.

\section{$\underline{\text { X-ray Crystal Structure Analysis of 6d }}$}

X-ray intensity data measurements of compound 6d was carried out on a Bruker SMART APEX II CCD diffractometer with graphite-monochromatized $\left(\mathrm{MoK}_{\alpha}=\right.$ $0.71073 \AA$ ) radiation. The X-ray generator was operated at $50 \mathrm{kV}$ and $30 \mathrm{~mA}$. A preliminary set of cell constants and an orientation matrix were calculated from three sets of 36 frames. Data were collected with $\omega$ scan width of $0.5^{\circ}$ at different settings of $\varphi$ and $2 \theta$ with a frame time of 10 secs keeping the sample-to-detector distance fixed at $5.00 \mathrm{~cm}$. The X-ray data collection was monitored by APEX2 program (Bruker, 2006). ${ }^{4}$ All the data were corrected for Lorentzian, polarization and absorption effects using SAINT and SADABS programs (Bruker, 2006). SHELX-97 was used for structure solution and full matrix least-squares refinement on $F^{2} \cdot 5$ All the hydrogen atoms

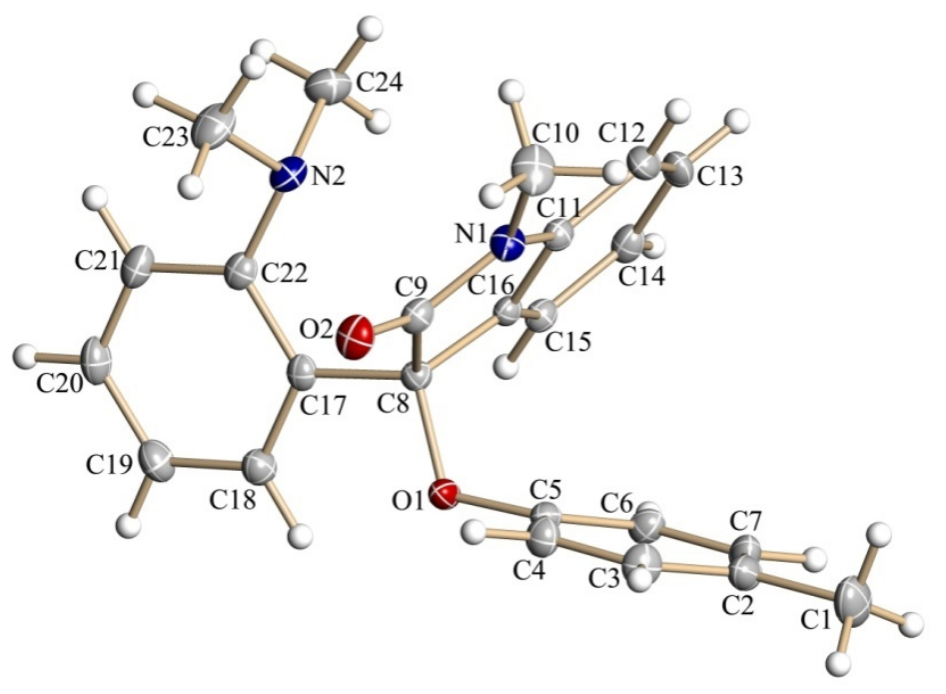


were placed in geometrically idealized position and constrained to ride on their parent atoms. An ORTEP III $^{6}$ view of both compounds were drawn with $30 \%$ probability displacement ellipsoids and $\mathrm{H}$ atoms are shown as small spheres of arbitrary radii.

Crystal data of $6 \mathbf{d} \mathrm{C}_{24} \mathrm{H}_{24} \mathrm{~N}_{2} \mathrm{O}_{2}, \mathrm{M}=372.45$, colorless block, $0.51 \times 0.48 \times 0.27 \mathrm{~mm}^{3}$, monoclinic, space group $P 2_{1} / c, a=9.0073(2) \AA, b=29.1800(7) \AA, c=7.8919$ (2) $\AA, \beta=$ 104.5870(10) $)^{\circ} V=2007.39(8) \AA^{3}, \mathrm{Z}=4, T=200(2) \mathrm{K}, 2 \theta_{\max }=54.00^{\circ}, D_{\text {calc }}\left(\mathrm{g} \mathrm{cm}^{-3}\right)=$ 1.232, $F(000)=792, \mu\left(\mathrm{mm}^{-1}\right)=0.079,20823$ reflections collected, 4382 unique reflections $\left(R_{\text {int }}=0.0323\right), 3764$ observed $(I>2 \sigma(I))$ reflections, multi-scan absorption correction, $T_{\min }=0.961, T_{\max }=0.979,334$ refined parameters, $S=1.112, R 1=0.0569$, $w R 2=0.1243$ (all data $R=0.0677, w R 2=0.1293$ ), maximum and minimum residual electron densities; $\Delta \rho_{\max }=0.45, \Delta \rho_{\min }=-0.23\left(\mathrm{e} \AA^{-3}\right)$.

\section{1-Benzyl-3-(2-(dimethylamino)phenyl)-3-phenoxyindolin-2-one (6e)}

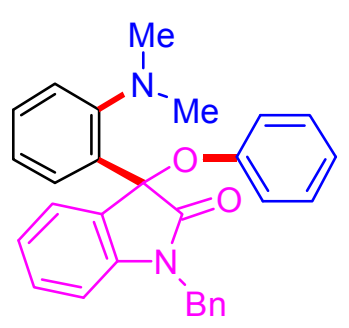

Following the general procedure, treatment of 2-(trimethylsilyl)phenyl trifluoromethanesulfonate 1a $(0.179 \mathrm{~g}, 146 \mu \mathrm{L}, 0.6 \mathrm{mmol})$ and 1benzylindoline-2,3-dione $\quad 5 d \quad(0.237 \quad \mathrm{~g}, \quad 1.0 \mathrm{mmol})$ with $\mathrm{N}, \mathrm{N}$ dimethylaniline 3b $(0.061 \mathrm{~g}, 64 \mu \mathrm{L}, 0.5 \mathrm{mmol})$ in the presence of $\mathrm{KF}$ $(0.070 \mathrm{~g}, 1.2 \mathrm{mmol})$ and [18]-crown-6 (0.317 g, $1.2 \mathrm{mmol})$ in THF (2.0 $\mathrm{mL}$ ) at $-10{ }^{\circ} \mathrm{C}$ to $\mathrm{rt}$ for $12 \mathrm{~h}$ followed by flash column chromatography (Pet. ether $/$ EtOAc $=$ 95/05) of the crude reaction mixture using silica gel afforded 1-benzyl-3-(2(dimethylamino)phenyl)-3-phenoxyindolin-2-one $6 \mathbf{e}$ as a orange solid $(0.120 \mathrm{~g}, 55 \%)$.

$\boldsymbol{R}_{\mathbf{f}}($ Pet. ether $/$ EtOAc $=80 / 20): 0.63 ;{ }^{1} \mathbf{H}$ NMR $\left(\mathbf{4 0 0} \mathbf{~ M H z}, \mathbf{C D C l}_{3}\right) \delta 8.23\left(\mathrm{dd}, J_{1}=7.4, J_{2}=1.8\right.$ $\mathrm{Hz}, 1 \mathrm{H}), 7.39-7.32(\mathrm{~m}, 2 \mathrm{H}), 7.24-7.21(\mathrm{~m}, 1 \mathrm{H}), 7.15-7.03(\mathrm{~m}, 8 \mathrm{H}), 6.93-6.89(\mathrm{~m}, 1 \mathrm{H}), 6.86-6.84$ (m, 2H), $6.67(\mathrm{~d}, J=6.8 \mathrm{~Hz}, 2 \mathrm{H}), 6.39(\mathrm{~d}, J=7.8 \mathrm{~Hz}, 1 \mathrm{H}), 5.26(\mathrm{~d}, J=15.9 \mathrm{~Hz}, 1 \mathrm{H}), 4.26(\mathrm{~d}, J=$ $15.9 \mathrm{~Hz}, 1 \mathrm{H}), 2.38$ (bs, 3H), 1.69 (bs, 3H). $\left.{ }^{13} \mathbf{C} \mathbf{~ N M R ~ ( 1 0 0 ~ M H z , ~} \mathbf{C D C l}_{3}\right) \delta$ 174.02, 154.61, $150.97,144.65,138.71,135.81,129.79,129.52$, 129.12, 128.96, 128.54, 127.47, 127.16, 126.92, $126.64,125.47,124.64,124.31,123.64,122.20,109.07,84.65,45.49,44.55,43.97$. HRMS (ESI) calculated $[\mathrm{M}+\mathrm{H}]^{+}$for $\mathrm{C}_{29} \mathrm{H}_{27} \mathrm{O}_{2} \mathrm{~N}_{2}: 435.2067$, found: 435.2064. FTIR ( $\left.\mathbf{c m}^{-1}\right): 3019,2936$, 2865, 2785, 1722, 1604, 1484, 1353, 1216, 934, 767, 675. 


\section{$N, N$-Dimethyl-2-(2,2,2-trifluoro-1-phenoxy-1-phenylethyl)aniline (8)}

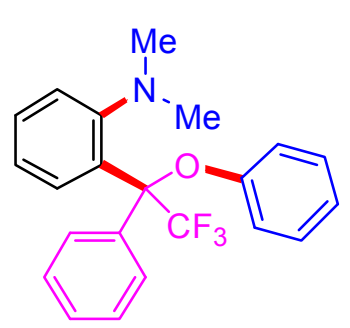

Following the general procedure $\mathbf{A}$, treatment of 2-(trimethylsilyl) phenyltrifluoromethanesulfonate 1a $(0.179 \mathrm{~g}, 146 \mu \mathrm{L}, 0.60 \mathrm{mmol})$ and 2,2,2-trifluoro-1-phenylethan-1-one $7(0.174 \mathrm{~g}, 140 \mu \mathrm{L}, 1.0 \mathrm{mmol})$ with $N, N$-dimethylaniline $3 \mathbf{b}(0.061 \mathrm{~g}, 64 \mu \mathrm{L}, 0.50 \mathrm{mmol})$ in the presence of $\mathrm{KF}(0.070 \mathrm{~g}, 1.20 \mathrm{mmol})$ and 18-crown-6 $(0.317 \mathrm{~g}, 1.20 \mathrm{mmol})$ in THF $(2.0 \mathrm{~mL})$ at $30^{\circ} \mathrm{C}$ for $12 \mathrm{~h}$ followed by flash column chromatography (Pet. ether/EtOAc $=$ 99.50/0.50) of the crude reaction mixture using silica gel afforded $N, N$-dimethyl-2-(2,2,2trifluoro-1-phenoxy-1-phenylethyl)aniline 8 as a colourless viscous oil ( $0.116 \mathrm{~g}, 62 \%$ yield). $\boldsymbol{R}_{\mathbf{f}}($ Pet. ether $/$ EtOAc $=97 / 03): 0.67 ;{ }^{1} \mathbf{H}$ NMR $\left(\mathbf{4 0 0} \mathbf{~ M H z}, \mathbf{C D C l}_{3}\right) \delta 7.98(\mathrm{~d}, J=7.7 \mathrm{~Hz}, 1 \mathrm{H})$, $7.59(\mathrm{~d}, J=6.4 \mathrm{~Hz}, 2 \mathrm{H}), 7.51(\mathrm{t}, J=7.5 \mathrm{~Hz}, 1 \mathrm{H}), 7.39-7.28(\mathrm{~m}, 5 \mathrm{H}), 7.15-7.11(\mathrm{~m}, 2 \mathrm{H}), 6.95(\mathrm{t}$, $J=7.3 \mathrm{~Hz}, 1 \mathrm{H}), 6.80(\mathrm{~d}, J=8.1 \mathrm{~Hz}, 2 \mathrm{H}), 1.86(\mathrm{~s}, 6 \mathrm{H}) .{ }^{13} \mathbf{C} \mathbf{~ N M R}\left(\mathbf{1 0 0} \mathbf{M H z}, \mathbf{C D C l}_{\mathbf{3}}\right) \delta 156.31$, $155.04,140.33,135.46,130.87,128.95-128.93$ (m), 128.83, 127.56, 127.45, 127.35, 126.62, 125.31, 123.73, 122.36, 120.08, 85.87-85.55 (m), 45.19. HRMS (ESI) calculated $[\mathrm{M}+\mathrm{H}]^{+}$for $\mathrm{C}_{22} \mathrm{H}_{21} \mathrm{ONF}_{3}$ : 372.1570, found: 372.1568. FTIR $\left(\mathbf{c m}^{-1}\right): 3065,3025,2937,2860,2826,2780$, $1590,1489,1453,1217,1165,1034,945,763,704,660$.

\section{2-(2-(Dimethylamino)phenyl)-2-phenoxy-1,2-diphenylethan-1-one (10)}

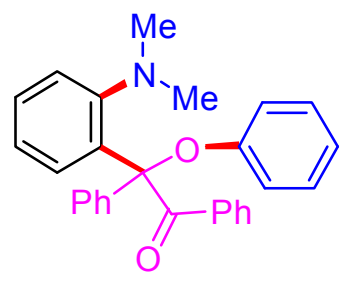

Following the general procedure A, treatment of 2-(trimethylsilyl)phenyl trifluoromethanesulfonate $1 \mathbf{a}(0.179 \mathrm{~g}, 146 \mu \mathrm{L}, 0.60 \mathrm{mmol})$ and benzil 9 $(0.210 \mathrm{~g}, 1.0 \mathrm{mmol})$ with $N, N$-dimethylaniline $3 \mathbf{b}(0.061 \mathrm{~g}, 64 \mu \mathrm{L}, 0.50$ $\mathrm{mmol})$ in the presence of $\mathrm{KF}(0.070 \mathrm{~g}, 1.20 \mathrm{mmol})$ and [18]-crown-6 $(0.317 \mathrm{~g}, 1.20 \mathrm{mmol})$ in THF $(2.0 \mathrm{~mL})$ at $-10{ }^{\circ} \mathrm{C}$ to $\mathrm{rt}$ for $12 \mathrm{~h}$ followed by flash column chromatography (Pet. ether $/$ EtOAc $=95 / 05)$ of the crude reaction mixture using silica gel afforded 2-(2-(dimethylamino)phenyl)-2-phenoxy-1,2-diphenylethan-1-one $\mathbf{1 0}$ as a yellow solid $(0.061 \mathrm{~g}, 30 \%)$.

$\boldsymbol{R}_{\mathbf{f}}($ Pet. ether $/$ EtOAc $=80 / 20): 0.70 ;{ }^{1} \mathbf{H}$ NMR (400 MHz, $\left.\mathbf{C D C l}_{3}\right) \delta 8.11(\mathrm{~d}, J=7.4 \mathrm{~Hz}, 2 \mathrm{H})$, $7.75(\mathrm{~d}, J=8.2 \mathrm{~Hz}, 3 \mathrm{H}), 7.47-7.31(\mathrm{~m}, 8 \mathrm{H}), 7.24-7.20(\mathrm{~m}, 1 \mathrm{H}), 7.07$ (t, $J=8 \mathrm{~Hz}, 2 \mathrm{H}), 6.85-6.80$ (m, 3H), 2.40 (s, 6H). ${ }^{13}$ C NMR (100 MHz, $\left.\mathbf{C D C l}_{3}\right) \delta 195.37,155.10,152.96,139.83,138.10$, $137.01,131.80,130.72,130.01,129.90,129.56,128.74,127.85,127.75,127.47,124.78,124.66$, 
121.06, 119.20, 91.85, 76.84, 45.10. HRMS (ESI) calculated $[\mathrm{M}+\mathrm{H}]^{+}$for $\mathrm{C}_{28} \mathrm{H}_{26} \mathrm{O}_{2} \mathrm{~N}: 408.1958$, found: 408.1956. FTIR $\left(\mathbf{c m}^{-1}\right)$ : 3021, 2941, 2833, 2787, 2231, 1595, 1492, 1224, $1022,760$.

\section{4-(Hydroxy(2-(methyl(phenyl)amino)phenyl)methyl)benzonitrile (16)}

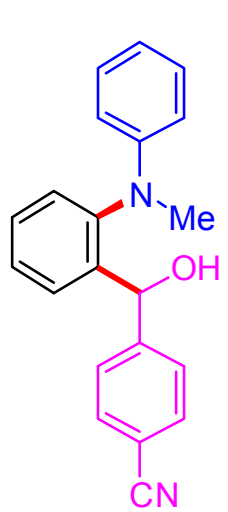

Following the general procedure $\mathbf{A}$, treatment of 2-(trimethylsilyl)phenyl trifluoromethanesulfonate $\mathbf{1 a}(0.179 \mathrm{~g}, \quad 146 \mu \mathrm{L}, \quad 0.60 \mathrm{mmol})$ and $4-$ formylbenzonitrile $2 \mathrm{a}(0.131 \mathrm{~g}, 1.0 \mathrm{mmol})$ with $N$-methylaniline $15(0.053 \mathrm{~g}, 54$ $\mu \mathrm{L}, 0.50 \mathrm{mmol})$ in the presence of $\mathrm{KF}(0.070 \mathrm{~g}, 1.20 \mathrm{mmol})$ and [18]-crown-6 $(0.317 \mathrm{~g}, 1.20 \mathrm{mmol})$ in THF $(2.0 \mathrm{~mL})$ at $-10^{\circ} \mathrm{C}$ to $\mathrm{rt}$ for $12 \mathrm{~h}$ followed by flash column chromatography (Pet. ether $/$ EtOAc $=80 / 20$ ) of the crude reaction mixture using silica gel to afford the two product as follows 4-(hydroxy(2(methyl(phenyl)amino)phenyl)methyl)benzonitrile 16 colourless viscous oil $(0.105 \mathrm{~g}, 67 \%)$ and $N$-methyl- $N$-phenylaniline $17^{3}$ colourless oil $(0.023 \mathrm{~g}, 25 \%)$.

$\boldsymbol{R}_{\mathbf{f}}($ Pet. ether $/$ EtOAc $=80 / 20): 0.35 ;{ }^{1} \mathbf{H}$ NMR $\left(400 \mathbf{~ M H z}, \mathbf{C D C l}_{3}\right) \delta$ 7.55-7.51 (m, 3H), 7.41$7.33(\mathrm{~m}, 4 \mathrm{H}), 7.19-7.12(\mathrm{~m}, 3 \mathrm{H}), 6.78(\mathrm{t}, J=7.3 \mathrm{~Hz}, 1 \mathrm{H}), 6.51(\mathrm{~d}, J=8.1 \mathrm{~Hz}, 2 \mathrm{H}), 5.93(\mathrm{~s}, 1 \mathrm{H})$, 3.23 (bs, 1H), 2.97 (s, 3H). ${ }^{13} \mathbf{C}$ NMR (100 MHz, $\mathbf{C D C l}_{3}$ ) $\delta$ 149.36, 149.01, 146.20, 141.34, 132.09, 130.10, 129.17, 128.69, 128.08, 127.39, 127.34, 118.89, 118.16, 113.73, 110.79, 71.68, 40.03. HRMS (ESI) calculated $[\mathrm{M}+\mathrm{H}]^{+}$for $\mathrm{C}_{21} \mathrm{H}_{19} \mathrm{ON}_{2}: 315.1492$, found: 315.1488 . FTIR (cm 1): 3455, 3021, 2244, 1598, 1494, 1218, 1033, 759, 669.

\section{Reaction of 2-phenyl-1,2,3,4-tetrahydroisoquinoline 19 with aryne generated from 1a and 4-formylbenzonitrile 2 a.}

Following the general procedure $\mathbf{A}$, treatment of 2-(trimethylsilyl)phenyl trifluoromethane sulfonate $1 \mathrm{a}(0.179 \mathrm{~g}, 146 \mu \mathrm{L}, 0.60 \mathrm{mmol})$ and 4-formylbenzonitrile $2 \mathrm{a}(0.131 \mathrm{~g}, 1.0 \mathrm{mmol})$ with 2-phenyl-1,2,3,4-tetrahydroisoquinoline $19(0.105 \mathrm{~g}, 0.50 \mathrm{mmol})$ in the presence of $\mathrm{KF}(0.070 \mathrm{~g}$, $1.20 \mathrm{mmol})$ and 18 -crown-6 $(0.317 \mathrm{~g}, 1.20 \mathrm{mmol})$ in THF $(2.0 \mathrm{~mL})$ at $-10{ }^{\circ} \mathrm{C}$ to $\mathrm{rt}$ for $12 \mathrm{~h}$ followed by flash column chromatography of the crude reaction mixture using silica gel afforded two product as follows 4-((2-(3,4-dihydroisoquinolin-2(1H)-yl)phenyl)(phenoxy)methyl) benzonitrile 20 as a yellow solid $(0.125 \mathrm{~g}, 60 \%$ yield) and 4-(hydroxy(2-(phenyl(2vinylbenzyl)amino)phenyl)methyl)benzonitrile 21 as a yellow viscous solid $(0.041 \mathrm{~g}, 20 \%$ yield). 


\section{4-((2-(3,4-Dihydroisoquinolin-2(1H)-yl)phenyl)(phenoxy)methyl)benzonitrile (20)}

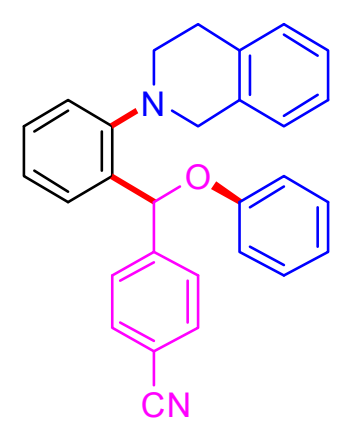

$\boldsymbol{R}_{\mathbf{f}}\left(\right.$ Pet. ether $/$ EtOAc $=$ 95/05): 0.48; ${ }^{1} \mathbf{H}$ NMR (400 $\left.\mathbf{M H z}, \mathbf{C D C l}_{3}\right) \delta 7.62$ $(\mathrm{d}, J=8.4 \mathrm{~Hz}, 2 \mathrm{H}), 7.58-7.53(\mathrm{~m}, 3 \mathrm{H}), 7.44-7.36(\mathrm{~m}, 2 \mathrm{H}), 7.28-7.20(\mathrm{~m}$, 6H), 7.01-6.97 (m, 5H), $4.19(\mathrm{~d}, J=15.0 \mathrm{~Hz}, 1 \mathrm{H}), 3.97$ (d, $J=15.0 \mathrm{~Hz}$, 1H), 3.37-3.31 (m, 1H), 3.20-3.14 (m, 1H), 3.07-2.93 (m, 2H). ${ }^{13} \mathbf{C}$ NMR (100 MHz, $\left.\mathbf{C D C l}_{3}\right) \delta 157.82,151.22,147.10,136.14,134.95,134.06$, $132.23,129.54,129.46,129.00,128.69,128.06,126.48,126.36,125.87$, 125.56, 121.89, 121.38, 118.87, 115.91, 111.25, 75.74, 56.08, 51.54, 29.80. HRMS (ESI) calculated $[\mathrm{M}+\mathrm{H}]^{+}$for $\mathrm{C}_{29} \mathrm{H}_{25} \mathrm{ON}_{2}$ : 417.1961, found: 417.1959. FTIR (cm $\left.{ }^{-1}\right): 3020,2924,2811$, 2743, 2230, 1729, 1595, 1492, 1457, 1378, 1222, 1024, 934, 768, 675.

\section{4-(Hydroxy(2-(phenyl(2-vinylbenzyl)amino)phenyl)methyl)benzonitrile (21)}

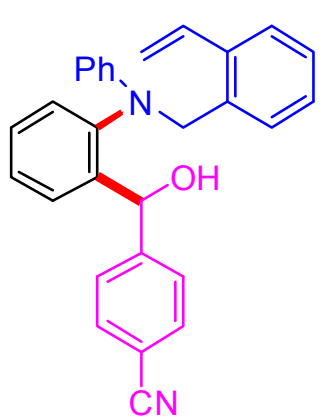

$\boldsymbol{R}_{\mathbf{f}}($ Pet. ether $/$ EtOAc $=80 / 20): 0.45 ;{ }^{1} \mathbf{H}$ NMR (400 $\left.\mathbf{M H z}, \mathbf{C D C l}_{3}\right) \delta 7.48$ $7.45(\mathrm{~m}, 3 \mathrm{H}), 7.37-7.33(\mathrm{~m}, 1 \mathrm{H}), 7.31-7.27(\mathrm{~m}, 3 \mathrm{H}), 7.25-7.13(\mathrm{~m}, 7 \mathrm{H})$, 6.84-6.77 (m, 2H), 6.58-6.56 (m, 2H), $5.91(\mathrm{~s}, 1 \mathrm{H}), 5.60\left(\mathrm{dd}, J_{l}=17.3 \mathrm{~Hz}\right.$, $\left.J_{2}=1.1 \mathrm{~Hz}, 1 \mathrm{H}\right), 5.30\left(\mathrm{dd}, J_{l}=11.0 \mathrm{~Hz}, J_{2}=1.1 \mathrm{~Hz}, 1 \mathrm{H}\right), 4.81(\mathrm{~d}, J=16.0$ $\mathrm{Hz}, 1 \mathrm{H}), 4.58(\mathrm{~d}, J=16.0 \mathrm{~Hz}, 1 \mathrm{H}), 2.09$ (bs, 1H). ${ }^{13} \mathbf{C}$ NMR (100 MHz, $\left.\mathbf{C D C l}_{3}\right) \delta 149.33,148.50,145.32,141.91,136.78,134.51,133.51,132.04$, $130.15,129.53,129.39,129.07,128.54,127.90,127.78,127.62,127.42,126.36,118.94,118.80$, 117.16, 114.85, 110.95, 70.50, 54.44. HRMS (ESI) calculated $[\mathrm{M}+\mathrm{H}]^{+}$for $\mathrm{C}_{29} \mathrm{H}_{25} \mathrm{ON}_{2}$ : 417.1961, found: 417.1956. FTIR (cm $\left.{ }^{-1}\right)$ : 3456, 3019, 2926, 2231, 1595, 1492, 1450, 1359, $1220,1027,918,852,765,680$.

\section{4-((2-((2(Dimethylamino)phenyl)(4-nitrophenyl)methoxy)phenyl)(phenoxy)methyl)benzoni} trile (22)

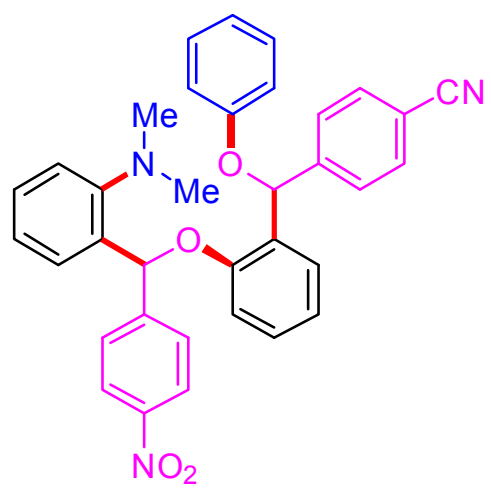

Following the general procedure B, treatment of 2(trimethylsilyl)phenyl trifluoromethanesulfonate 1a $(0.090 \mathrm{~g}, 73$ $\mu \mathrm{L}, 0.30 \mathrm{mmol})$ and 4-nitrobenzaldehyde $\mathbf{2 h}(0.076 \mathrm{~g}, 0.50$ mmol) with 4-((2-(dimethylamino)phenyl)(phenoxy)methyl) benzonitrile $4 \mathbf{b}(0.082 \mathrm{~g}, 0.25 \mathrm{mmol})$ in the presence of $\mathrm{KF}$ $(0.035 \mathrm{~g}, 0.60 \mathrm{mmol})$ and 18 -crown-6 $(0.159 \mathrm{~g}, 0.60 \mathrm{mmol})$ in 
THF $(1.0 \mathrm{~mL})$ at $-10{ }^{\circ} \mathrm{C}$ to $\mathrm{rt}$ for $12 \mathrm{~h}$ followed by flash column chromatography (Pet. ether/EtOAc $=95 / 05)$ of the crude reaction mixture using silica gel afforded 4-((2((2(Dimethylamino)phenyl)(4-nitrophenyl)methoxy) phenyl)(phenoxy)methyl) benzonitrile 22 as a yellow viscous oil ( $0.039 \mathrm{~g}, 28 \%$ yield) in 1:1 diastereomeric ratio.

$\boldsymbol{R}_{\mathbf{f}}($ Pet. ether $/$ EtOAc $=95 / 05): 0.52 ;{ }^{1} \mathbf{H}$ NMR $\left(\mathbf{5 0 0} \mathbf{~ M H z}, \mathbf{C D C l}_{3}\right) \delta 8.18-8.13(\mathrm{~m}, 4 \mathrm{H}), 7.62(\mathrm{~d}$, $J=8.2 \mathrm{~Hz}, 2 \mathrm{H}), 7.56-7.54(\mathrm{~m}, 4 \mathrm{H}), 7.50(\mathrm{~d}, J=8.2 \mathrm{~Hz}, 2 \mathrm{H}), 7.44-7.42(\mathrm{~m}, 3 \mathrm{H}), 7.40-7.25(\mathrm{~m}$, 10H), 7.23-7.14 (m, 6H), 7.09-7.04 (m, 3H), 7.01-6.93 (m, 6H), 6.90 (t, $J=8.4 \mathrm{~Hz}, 2 \mathrm{H}), 6.86$ (d, $J=8.1 \mathrm{~Hz}, 2 \mathrm{H}), 6.71(\mathrm{~s}, 1 \mathrm{H}), 6.61(\mathrm{~s}, 1 \mathrm{H}), 2.67$ (s, 6H), 2.57 (s, 6H). ${ }^{13} \mathbf{C}$ NMR (125 MHz, $\left.\mathbf{C D C l}_{3}\right) \delta 157.80,157.74,154.62,154.46,152.71,152.42,148.73,148.50,147.37,146.23$, $146.06,134.85,134.74,132.25,132.19,129.76,129.67,129.59,129.55,128.72,128.53,128.42$, $128.14,127.87,127.81,127.74,125.10,124.97,123.86,123.72,121.68,121.66,121.48,121.22$, $121.09,118.83,115.98,115.86,113.31,113.10,111.54,111.35,75.99,75.62,75.40,75.38$, 45.95, 45.80. HRMS (ESI) calculated $[\mathrm{M}+\mathrm{H}]^{+}$for $\mathrm{C}_{35} \mathrm{H}_{30} \mathrm{O}_{4} \mathrm{~N}_{3}$ : 556.2231, found: 556.2233. FTIR $\left(\mathbf{c m}^{-1}\right)$ : 3068, 3023, 2933, 2861, 2787, 2229, 1596, 1522, 1490, 1454, 1345, 1230, 1108, $1015,846,754$. 


\section{8. ${ }^{1} \mathrm{H}$ and ${ }^{13} \mathrm{C}$ NMR Spectra of 2-Amino Benzhydrol Derivatives}

\section{4-((4-Bromophenoxy)(2-(dimethylamino)phenyl)methyl)benzonitrile (4a)}

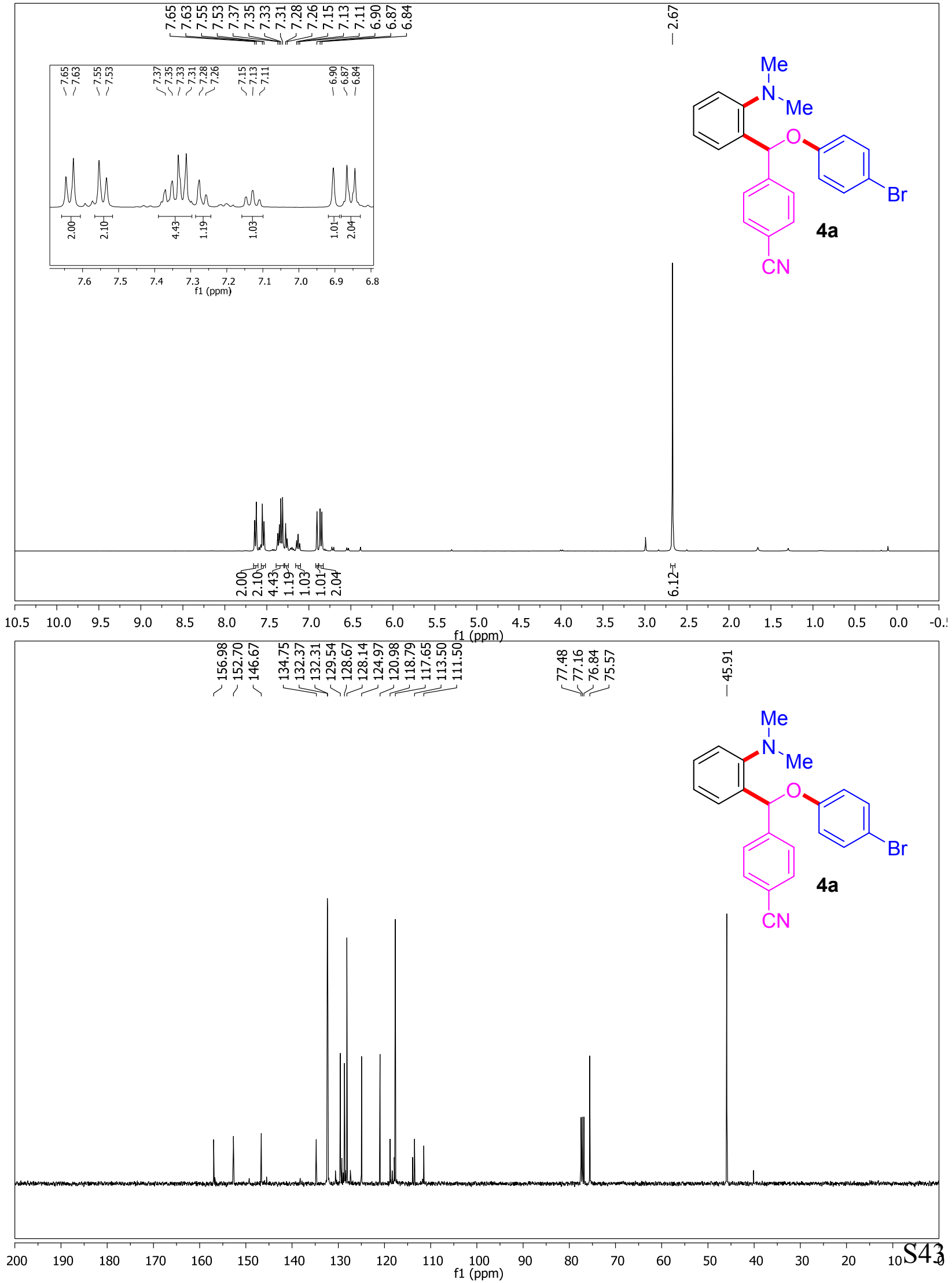




\section{4-((2-(Dimethylamino)phenyl)(phenoxy)methyl)benzonitrile (4b)}
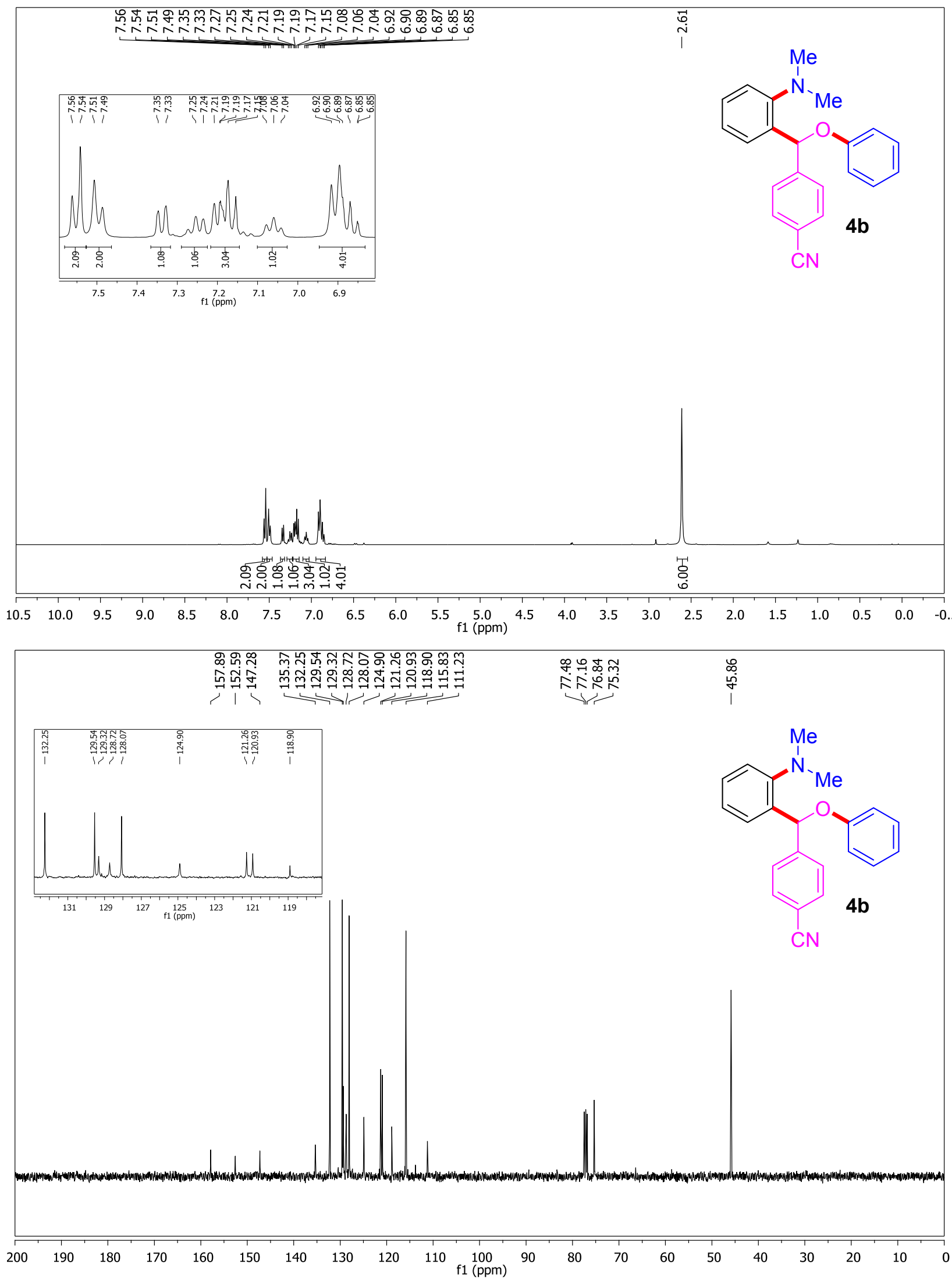


\section{4-((2-(Dimethylamino)phenyl)(p-tolyloxy)methyl)benzonitrile (4c)}

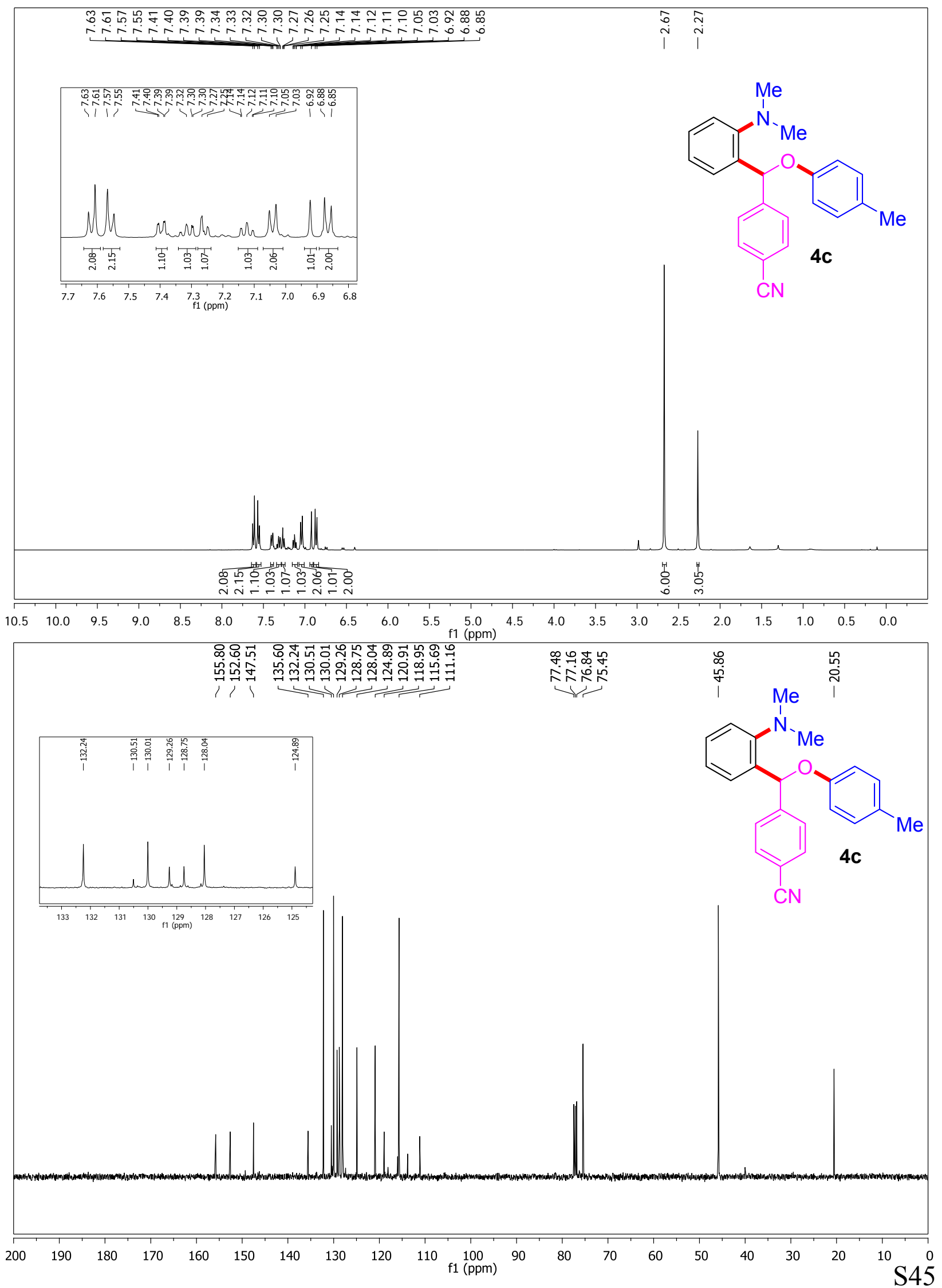


Ethyl-4-((4-cyanophenyl)(2-(dimethylamino)phenyl)methoxy)benzoate (4d)

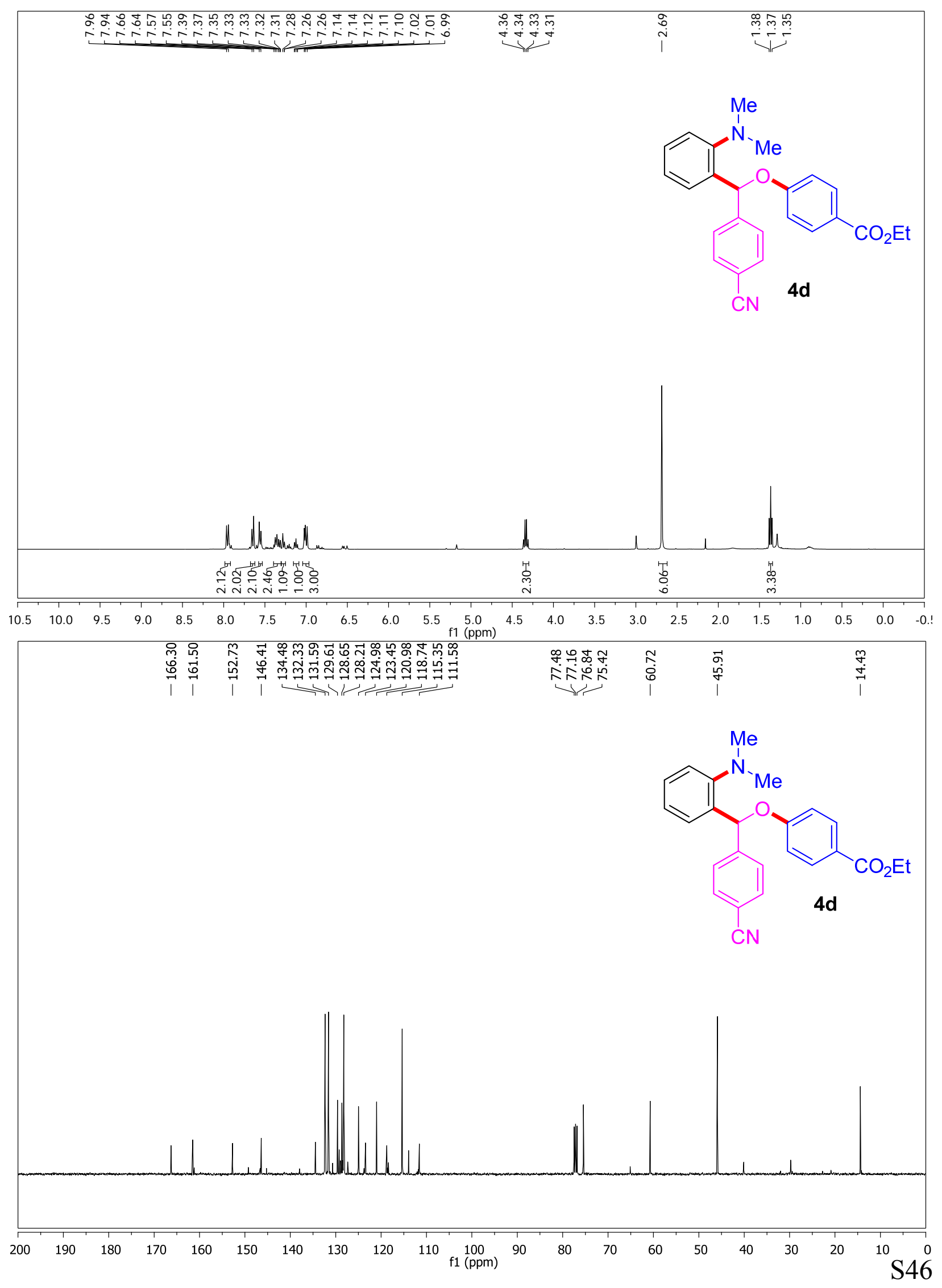




\section{4-((2-(Dimethylamino)phenyl)(m-tolyloxy)methyl)benzonitrile (4e)}
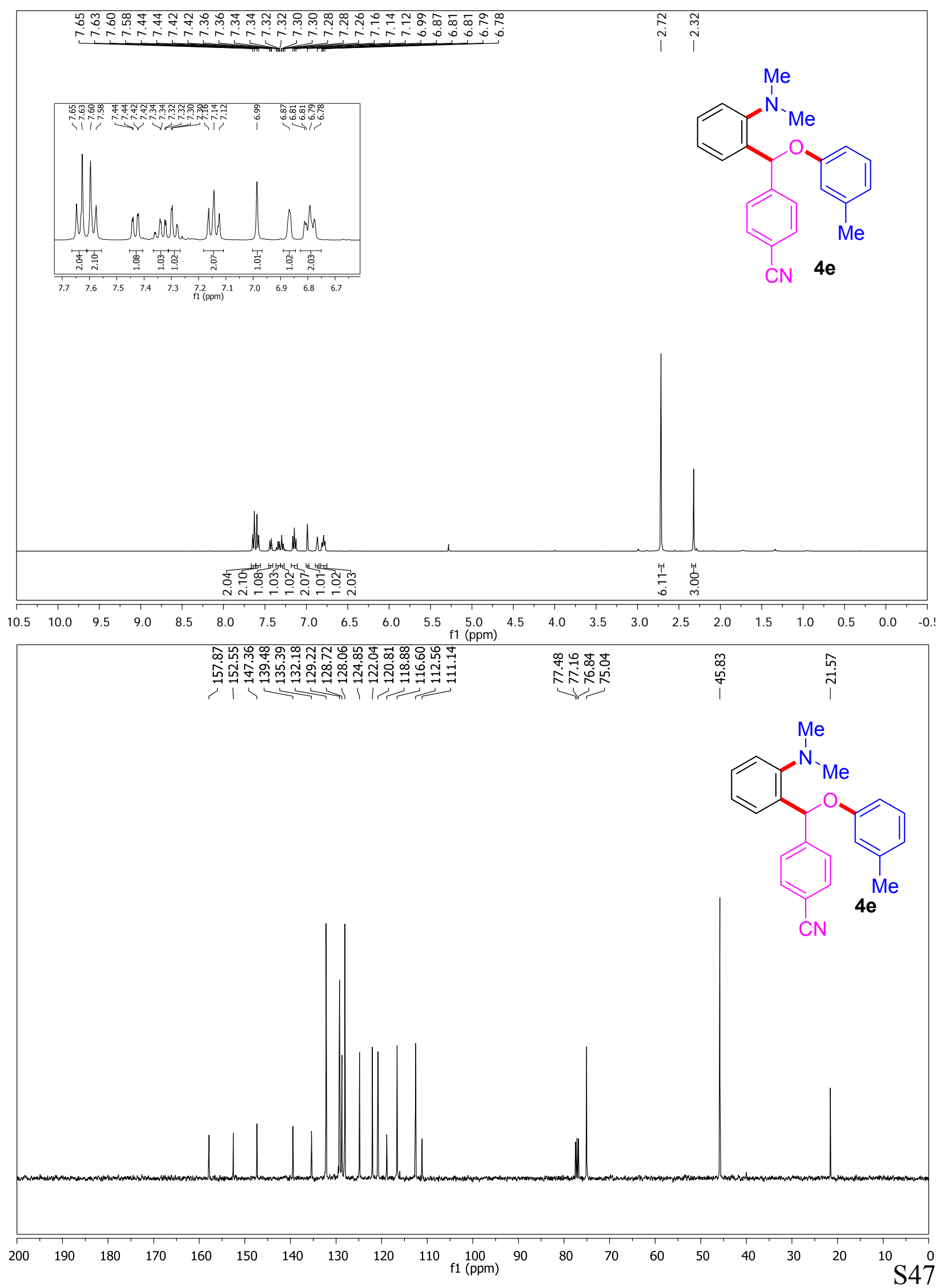
4-((3-Bromophenoxy)(2-(dimethylamino)phenyl)methyl)benzonitrile (4f)
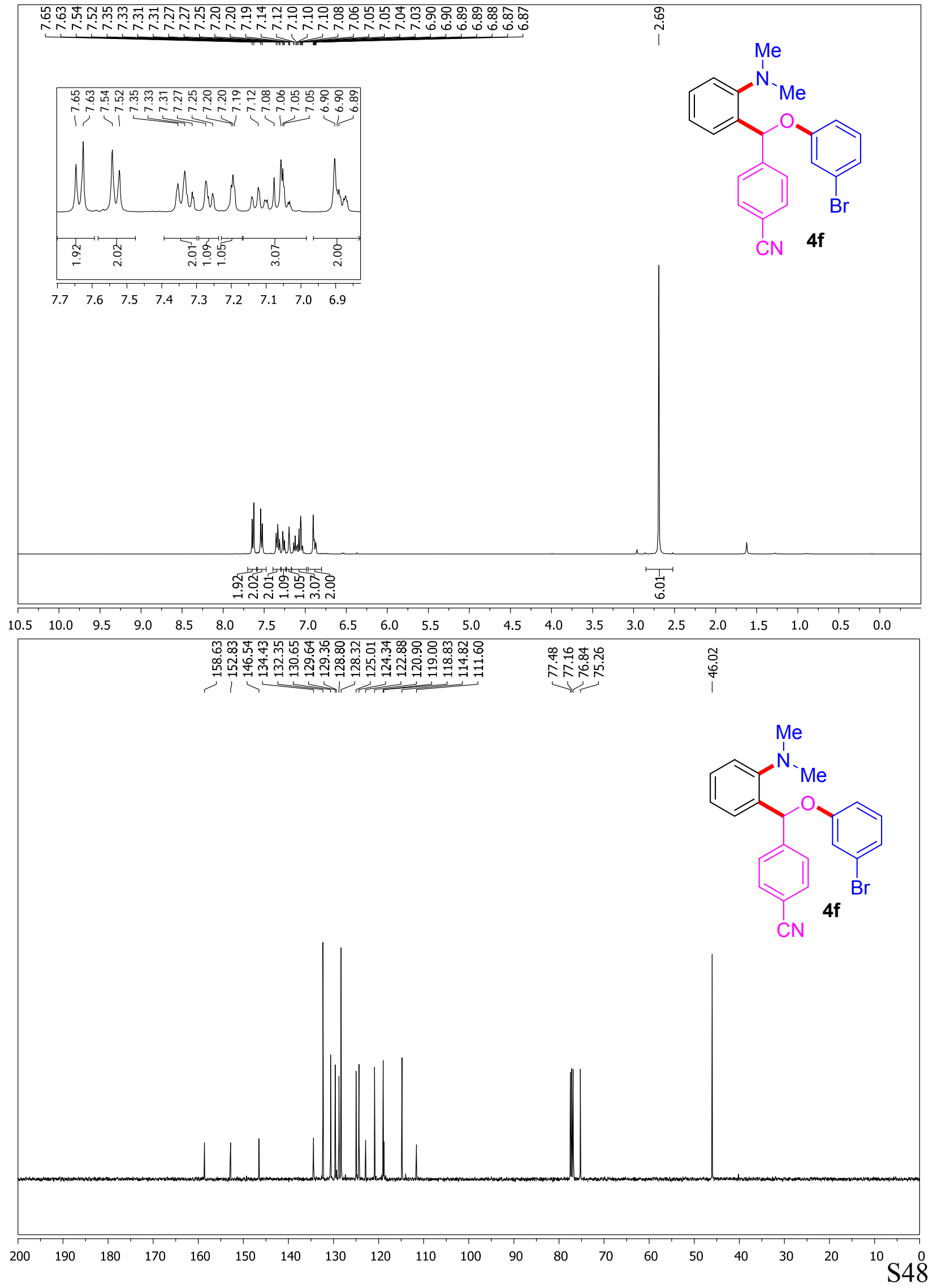


\section{4-((2-(Diethylamino)phenyl)(phenoxy)methyl)benzonitrile (4g)}
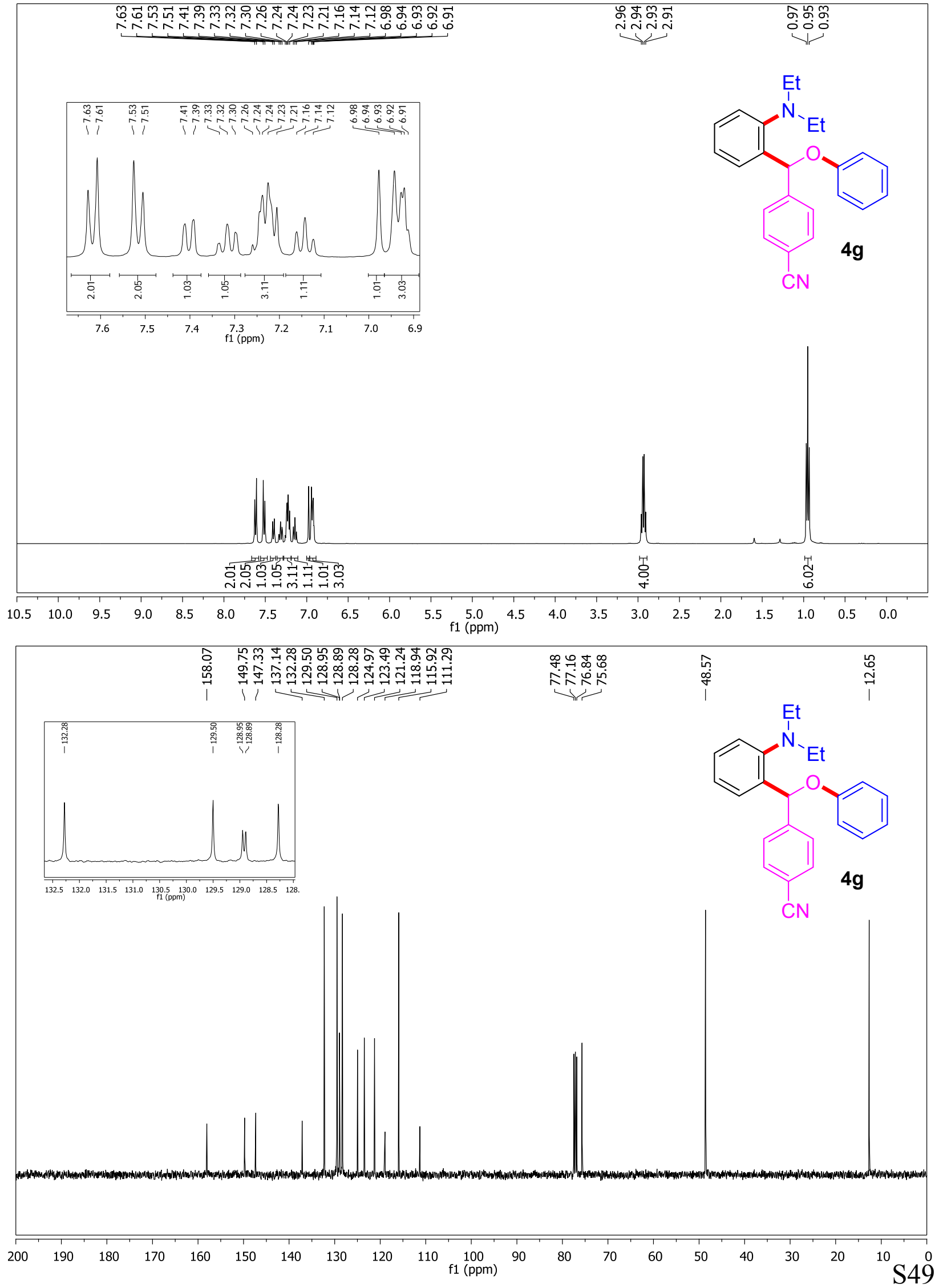


\section{4-((2-(Dibutylamino)phenyl)(phenoxy)methyl)benzonitrile (4h)}

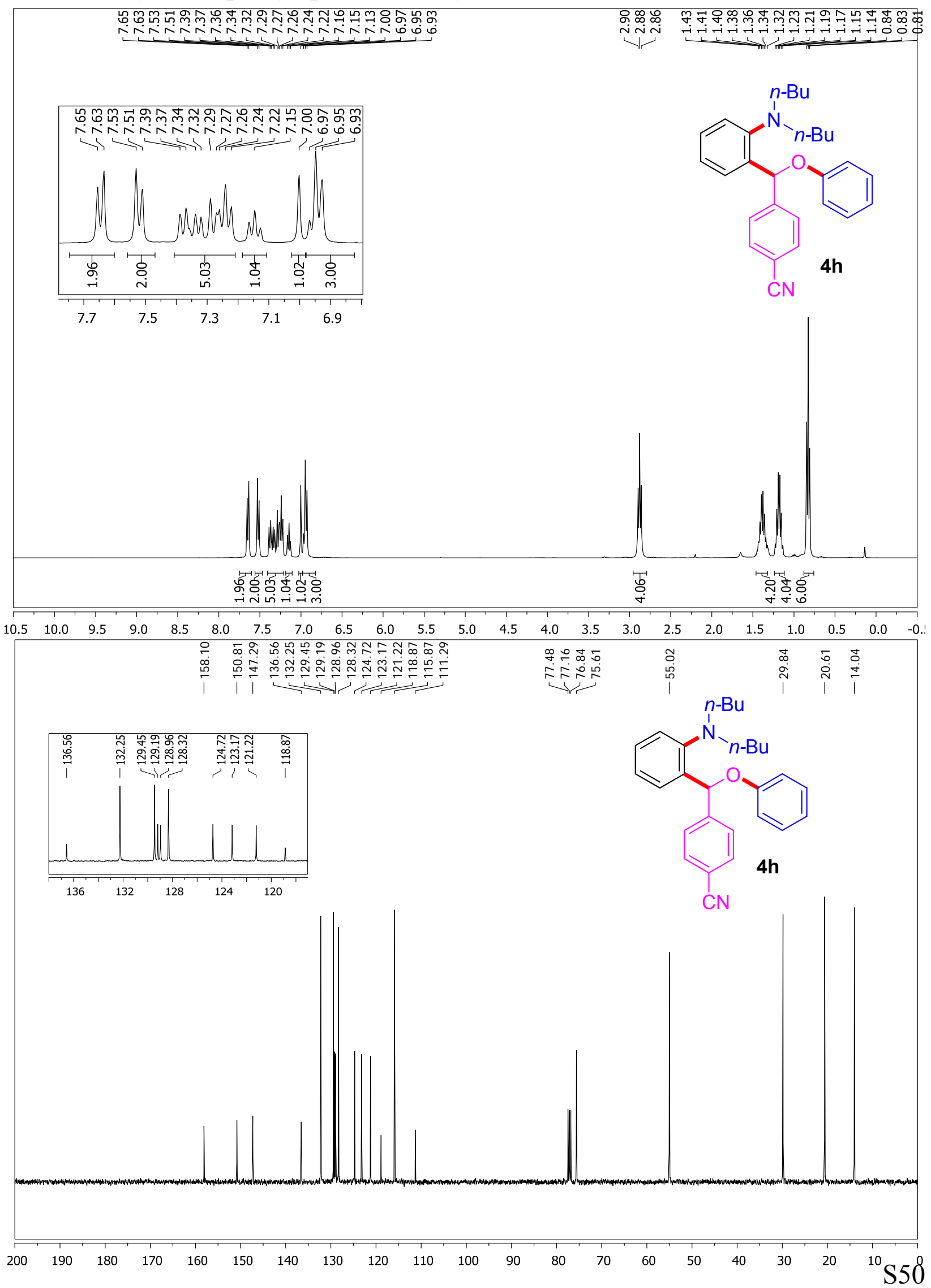




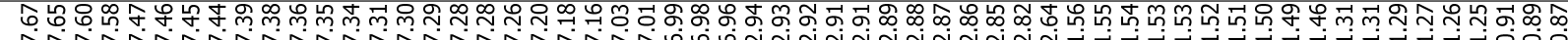

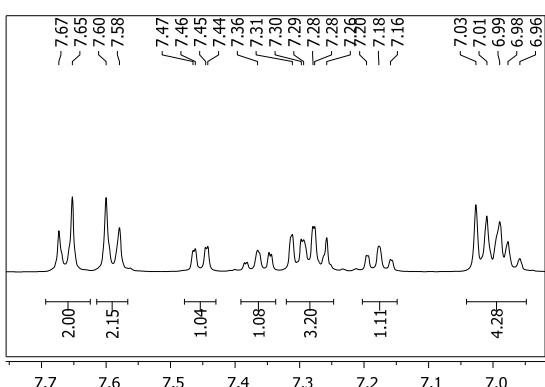<smiles>CCCCN(C)c1ccccc1C(Oc1ccccc1)c1ccc(C#N)cc1</smiles>

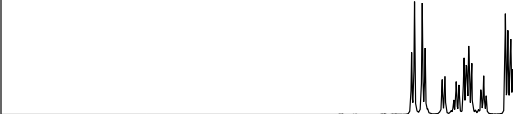

U.

$M$

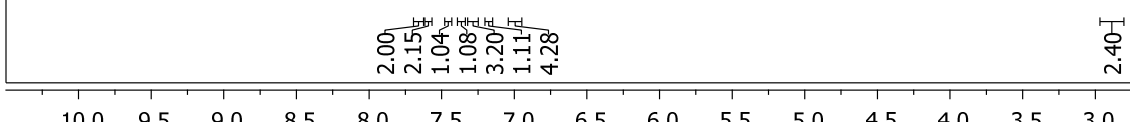

앙 $\stackrel{m}{m}$

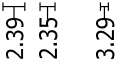

$\begin{array}{lllllllllll}10.0 & 9.5 & 9.0 & 8.5 & 8.0 & 7.5 & 7.0 & 6.5 & 6.0 & 5.5 & 5.0\end{array}$

ஸึ่

$<1>2$
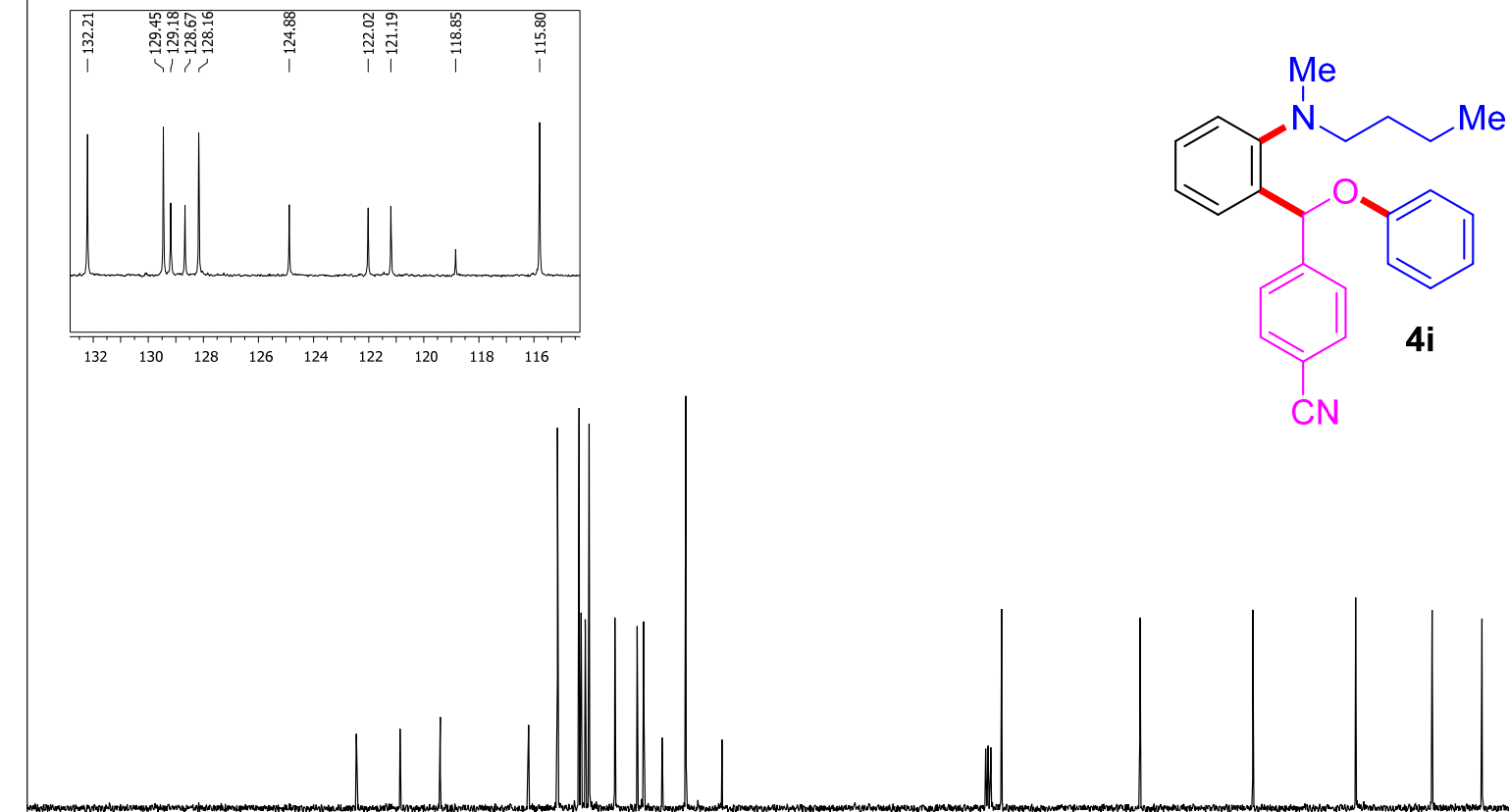

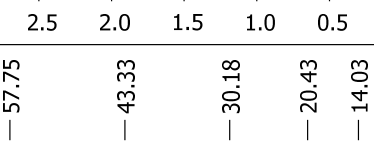

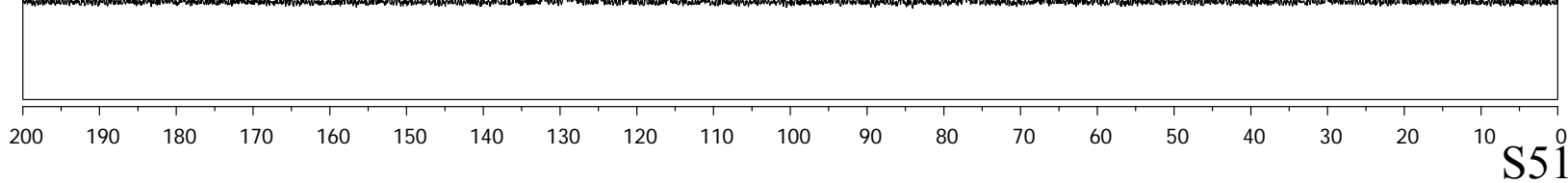




\section{4-((2-(Benzyl(methyl)amino)phenyl)(phenoxy)methyl)benzonitrile (4j)}

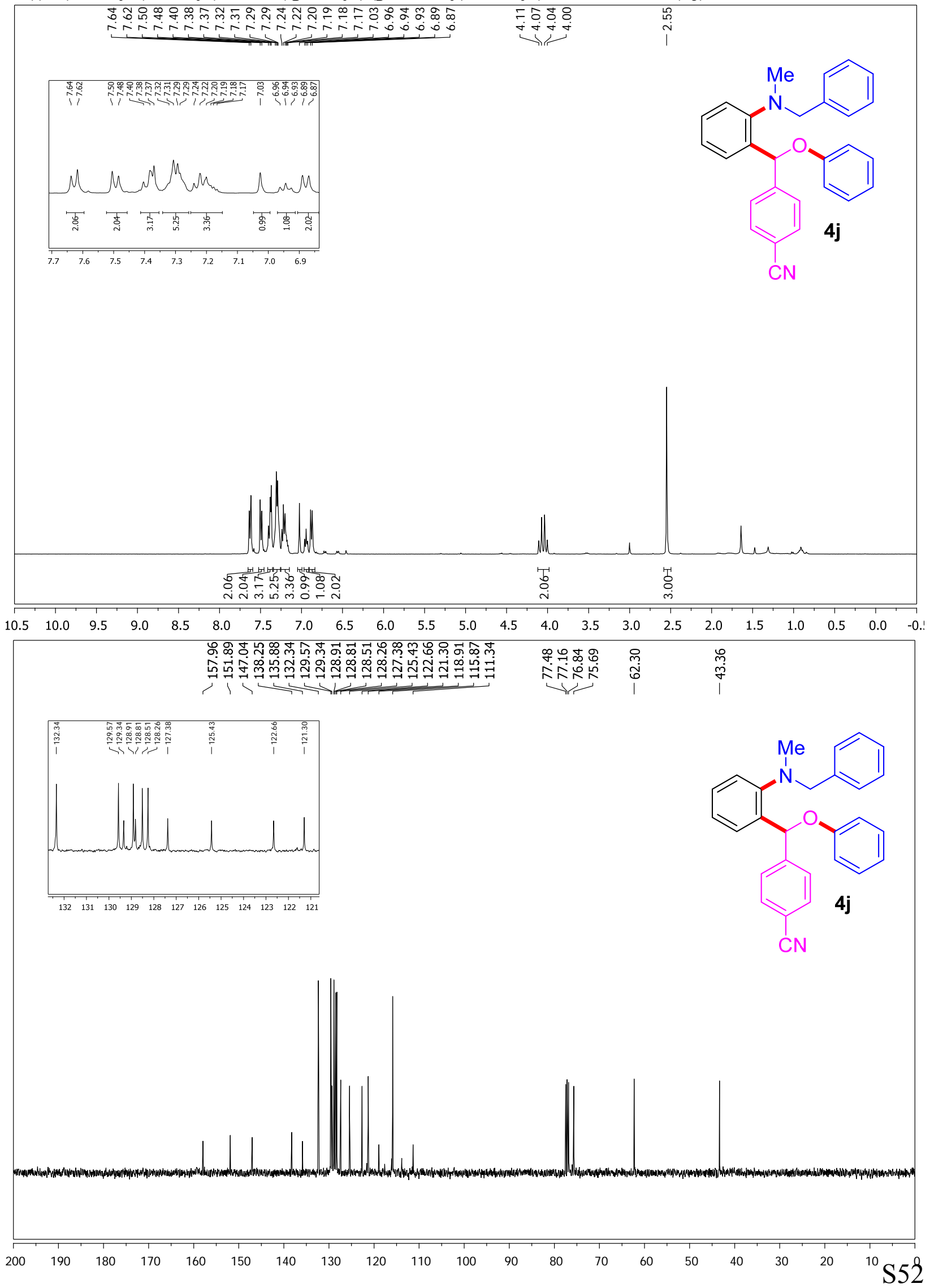




\section{4-((2-(Dimethylamino)phenyl)(naphthalen-1-yloxy)methyl)benzonitrile (4k)}
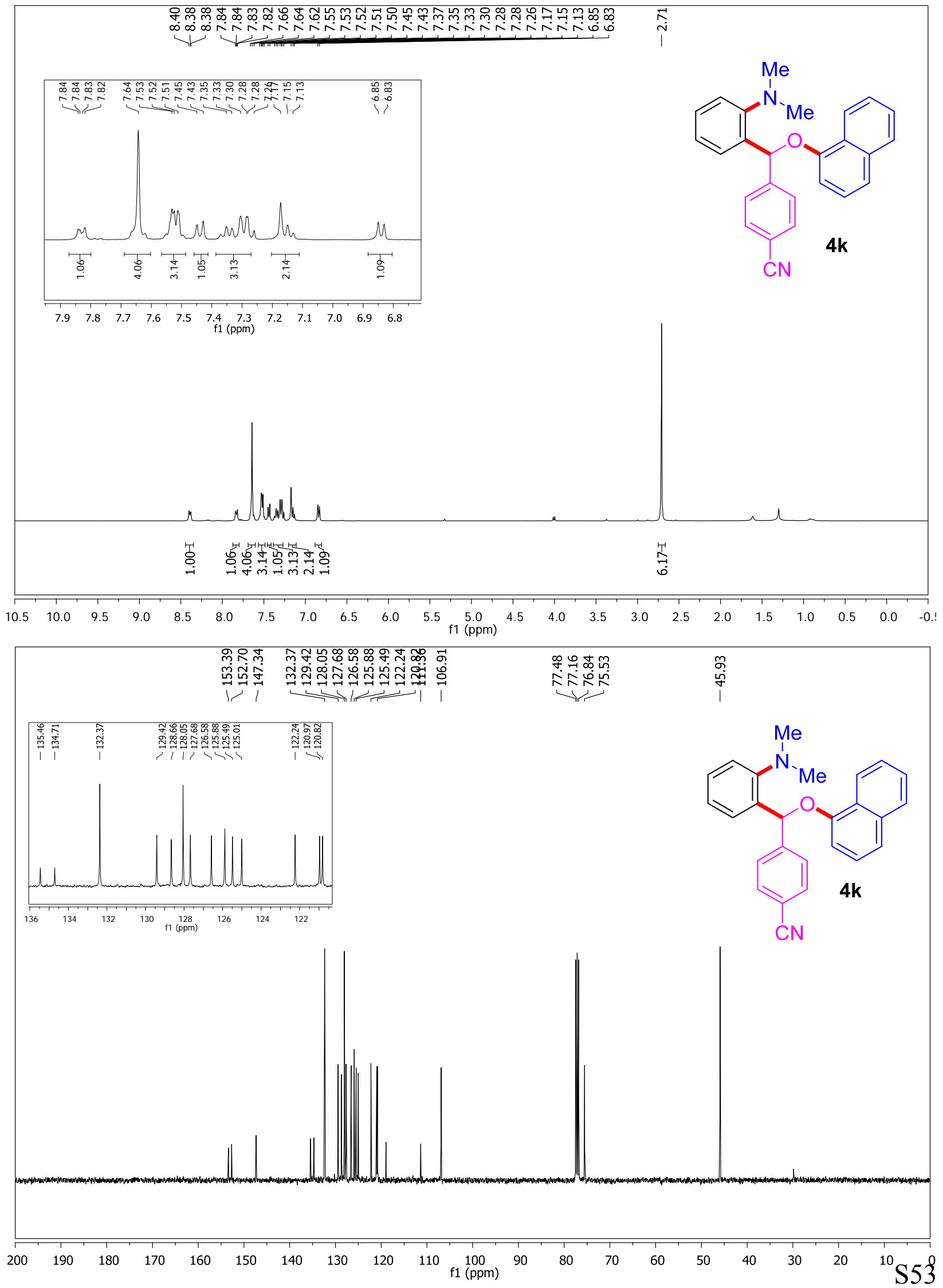
(E)-4-((4-(2-(5-Bromothiophen-2-yl)vinyl)phenoxy)(2-(dimethylamino)phenyl)methyl) benzonitrile (4l)

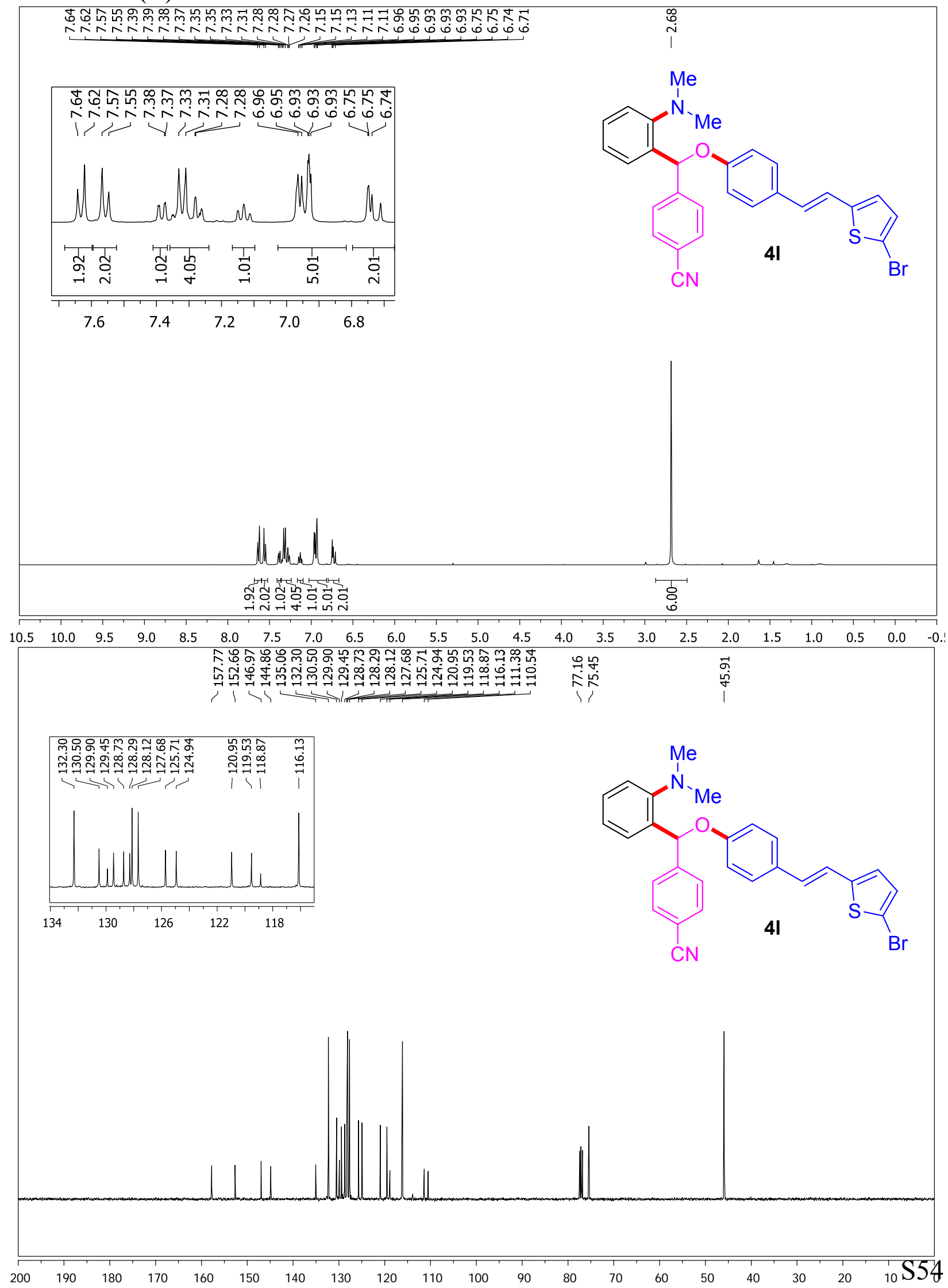


Ethyl (E)-3-(4-((4-cyanophenyl)(2-(dimethylamino)phenyl)methoxy)phenyl)acrylate (4m)

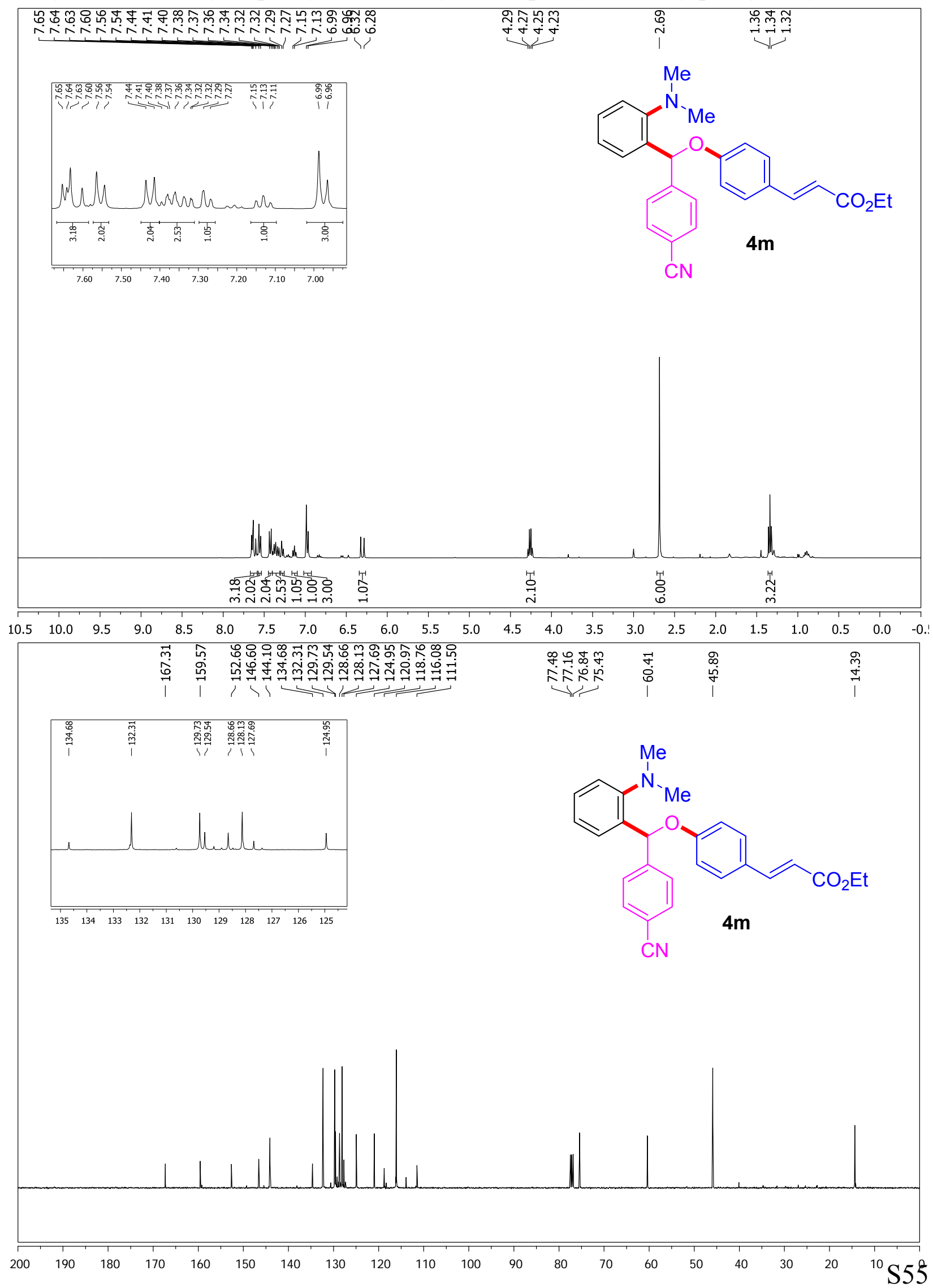




\section{Compound (4n)}

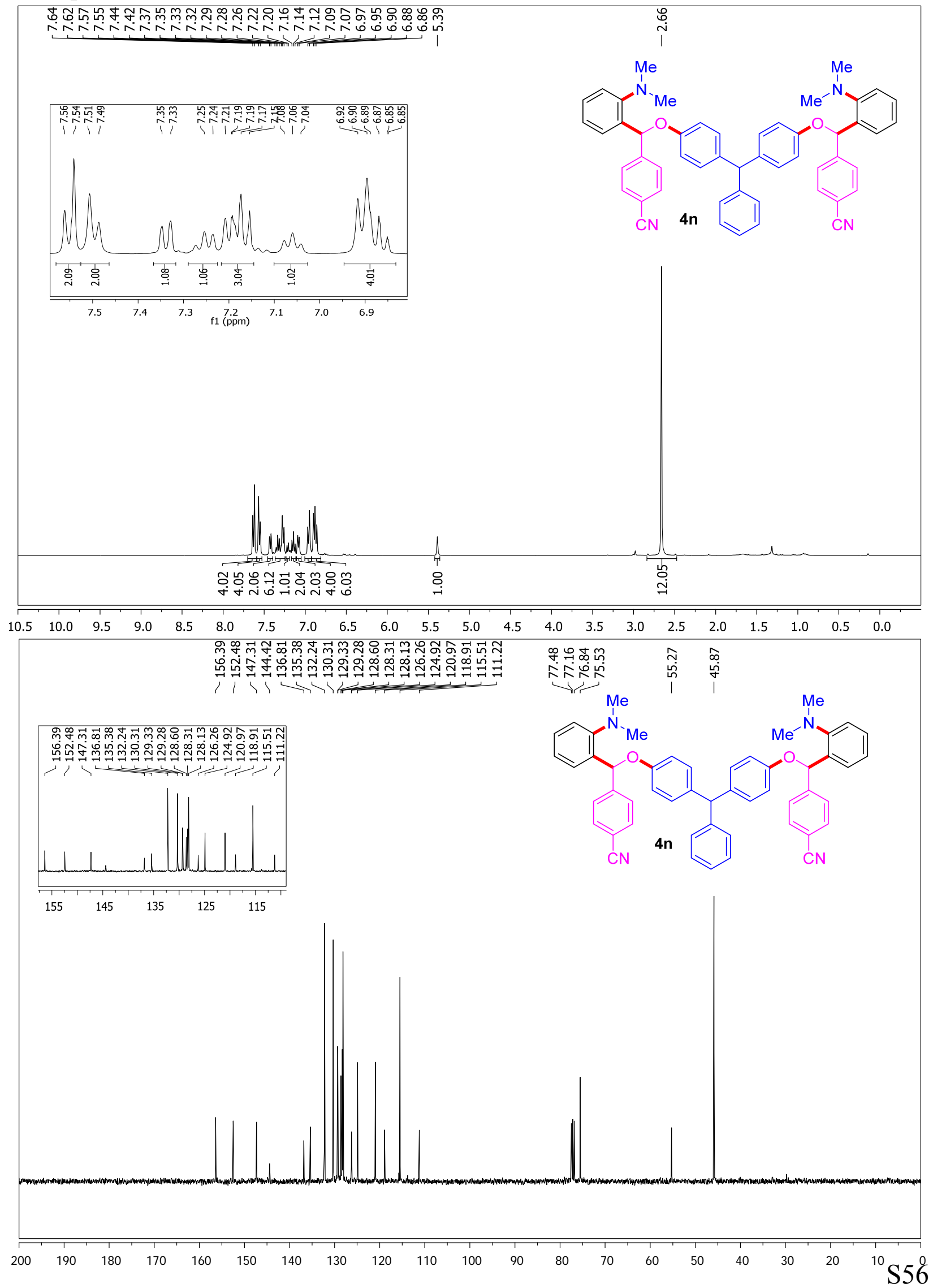




\section{4-((2-(Dimethylamino)phenyl)(3,5-dimethylphenoxy)methyl)benzonitrile (4o)}

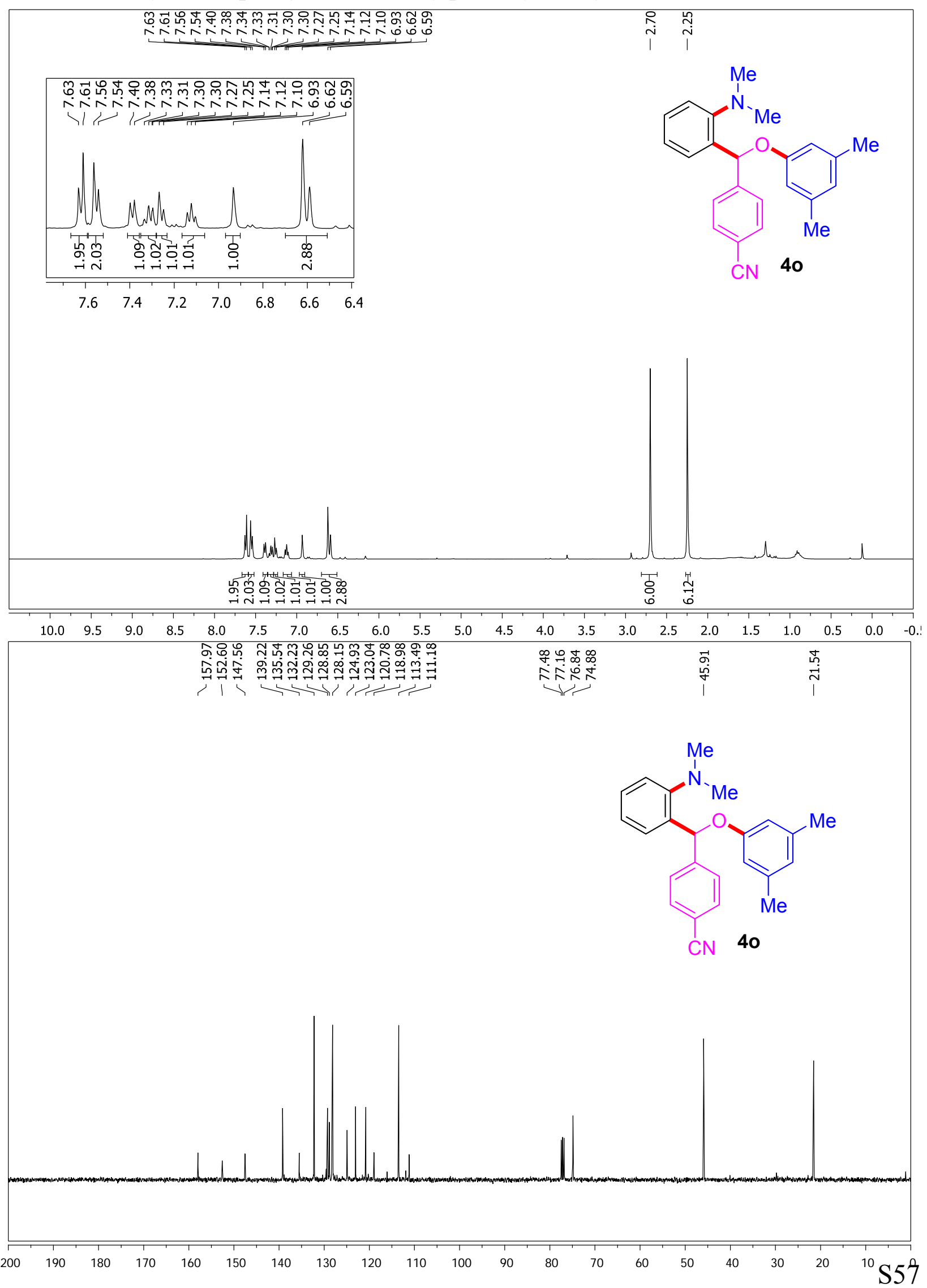


4-((Benzo[d][1,3]dioxol-5-yloxy)(2-(dimethylamino)phenyl)methyl)benzonitrile (4p)

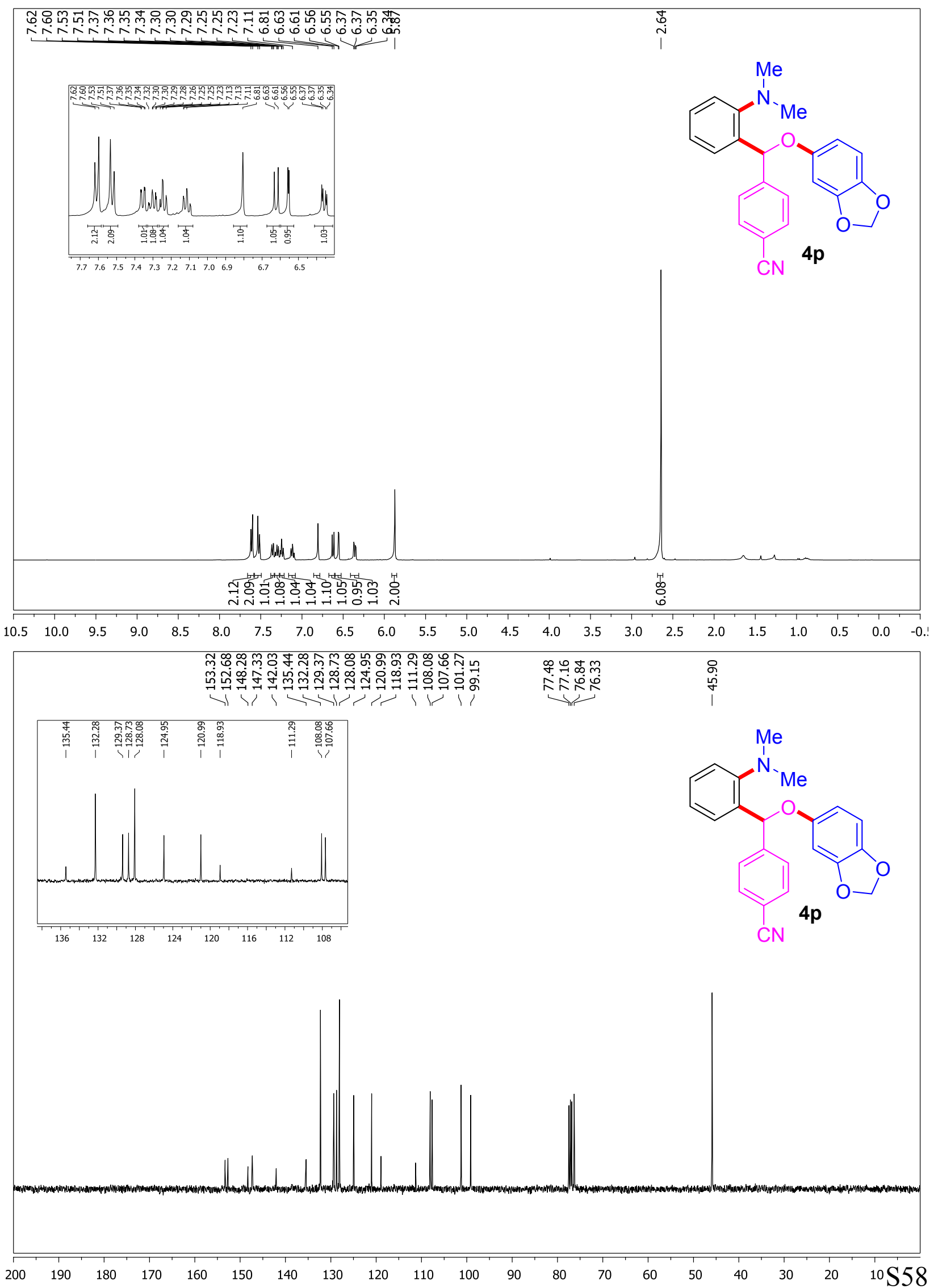




\section{$N, N$-Dimethyl-2-(phenoxy(phenyl)methyl)aniline (4q)}

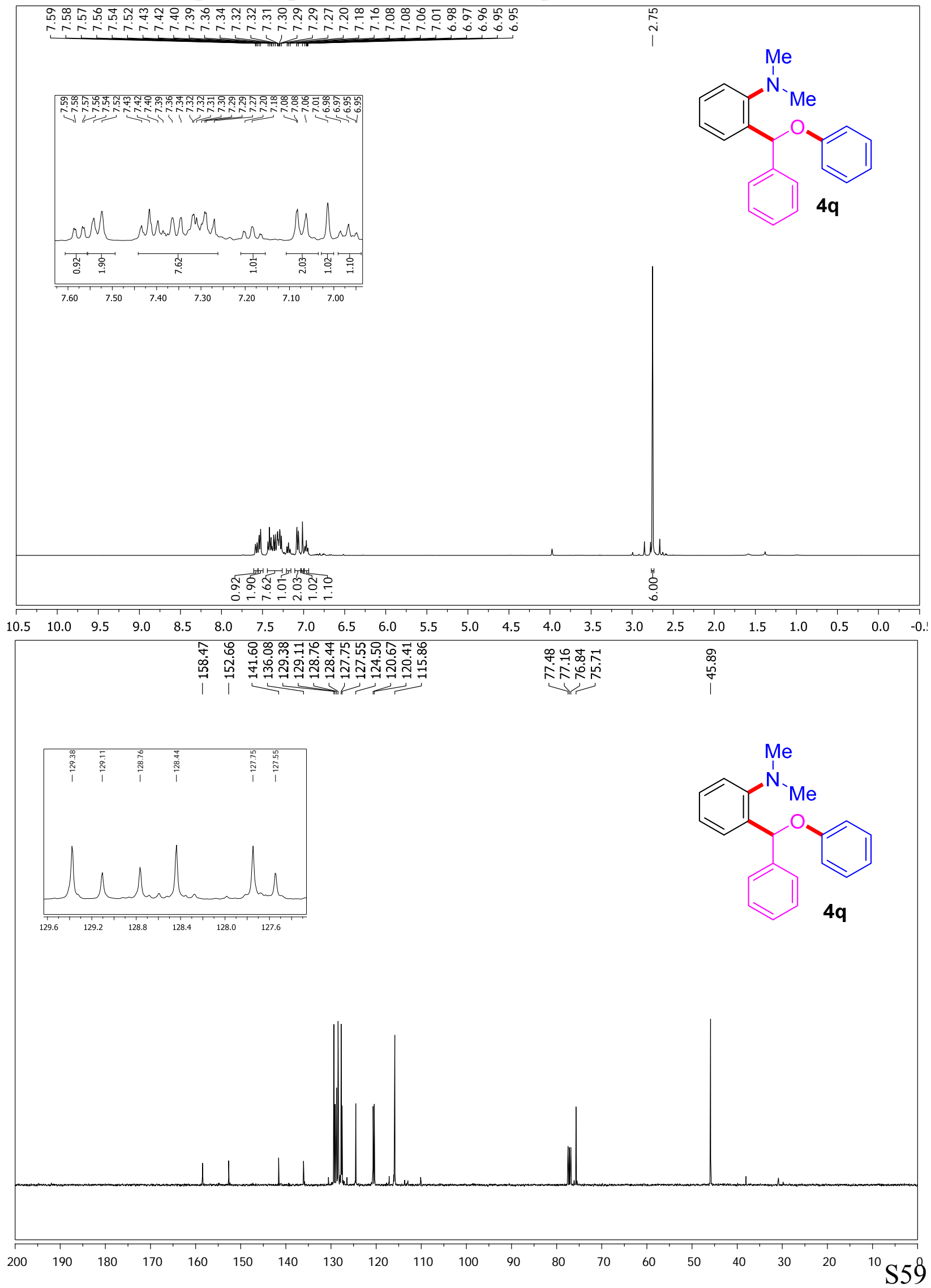




\section{2-((4-Bromophenyl)(phenoxy)methyl)- $N, N$-dimethylaniline (4r)}
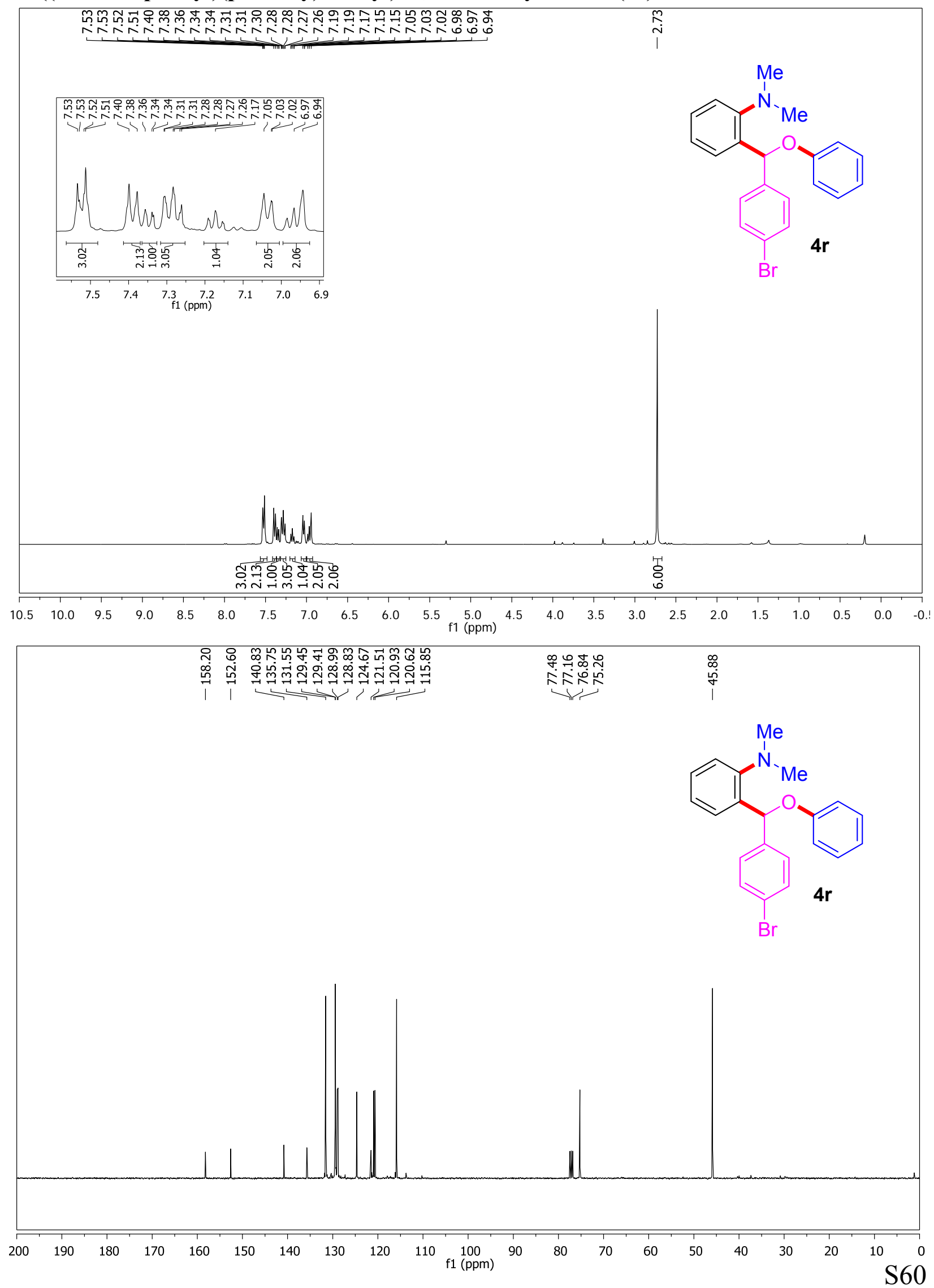
2-((4-Chlorophenyl)(phenoxy)methyl)- $N, N$-dimethylaniline (4s)
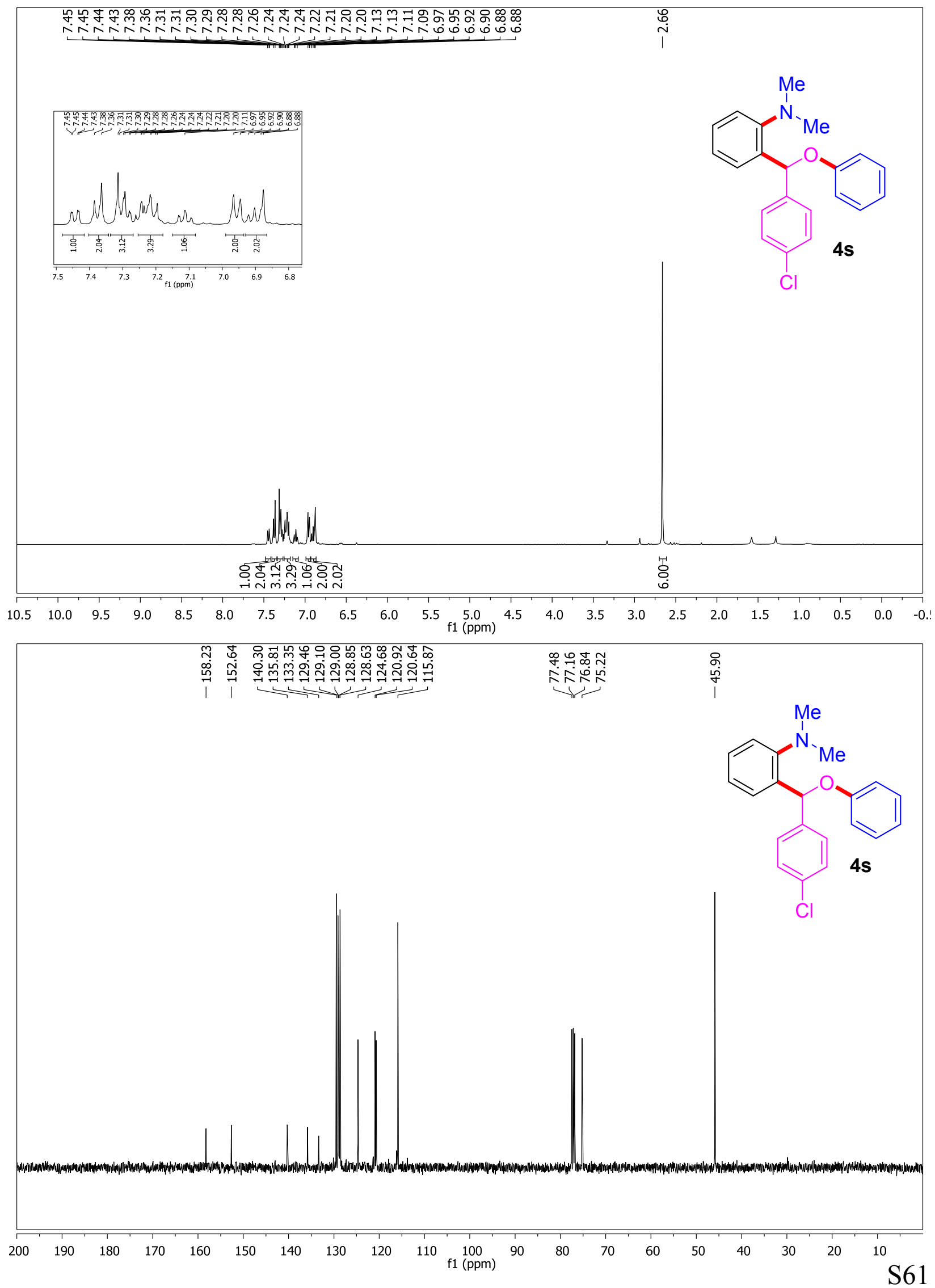


\section{2-((4-Fluorophenyl)(phenoxy)methyl)- $N, N$-dimethylaniline (4t)}
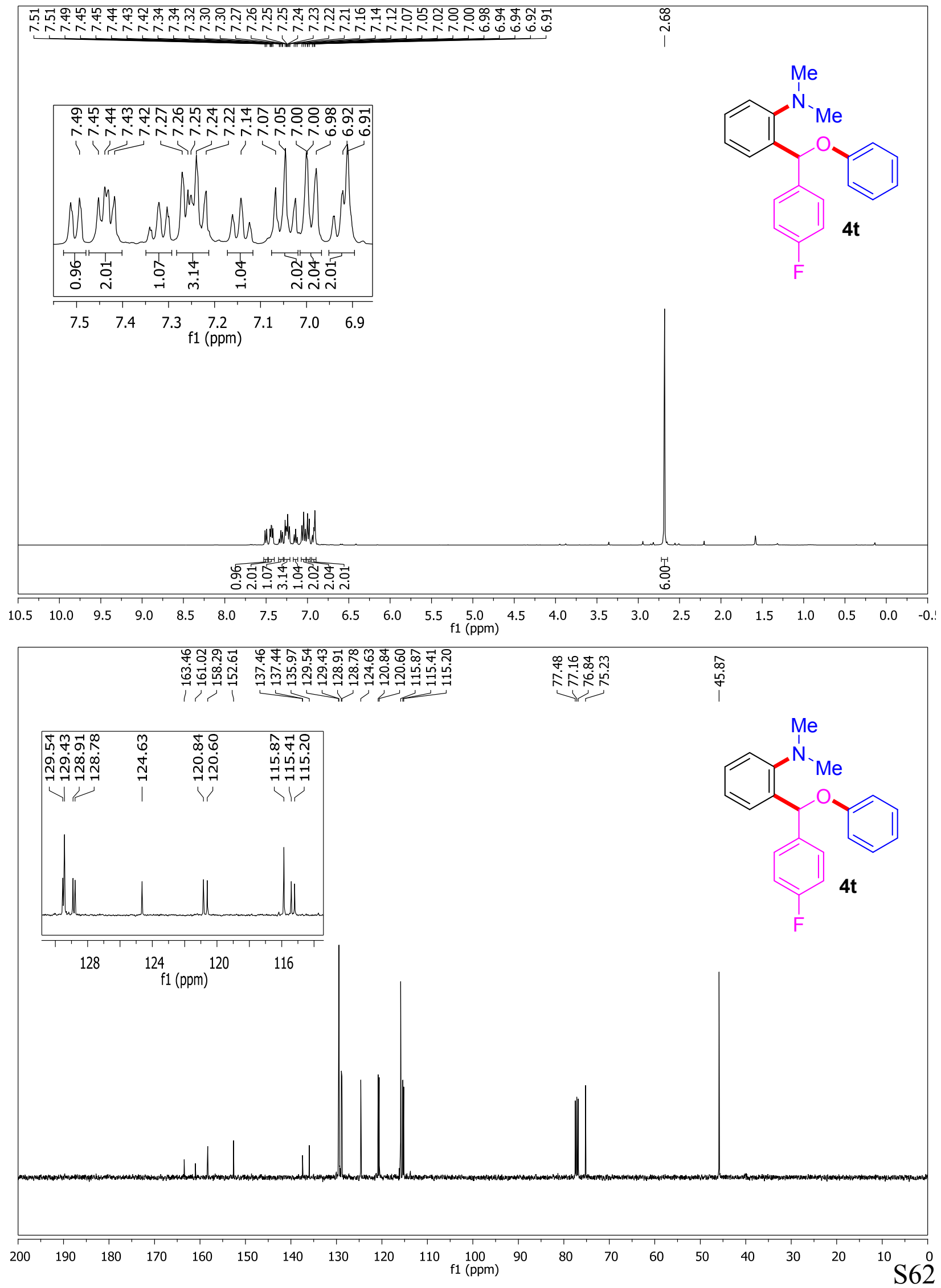
Methyl-4-((2-(dimethylamino)phenyl)(phenoxy)methyl)benzoate (4u)

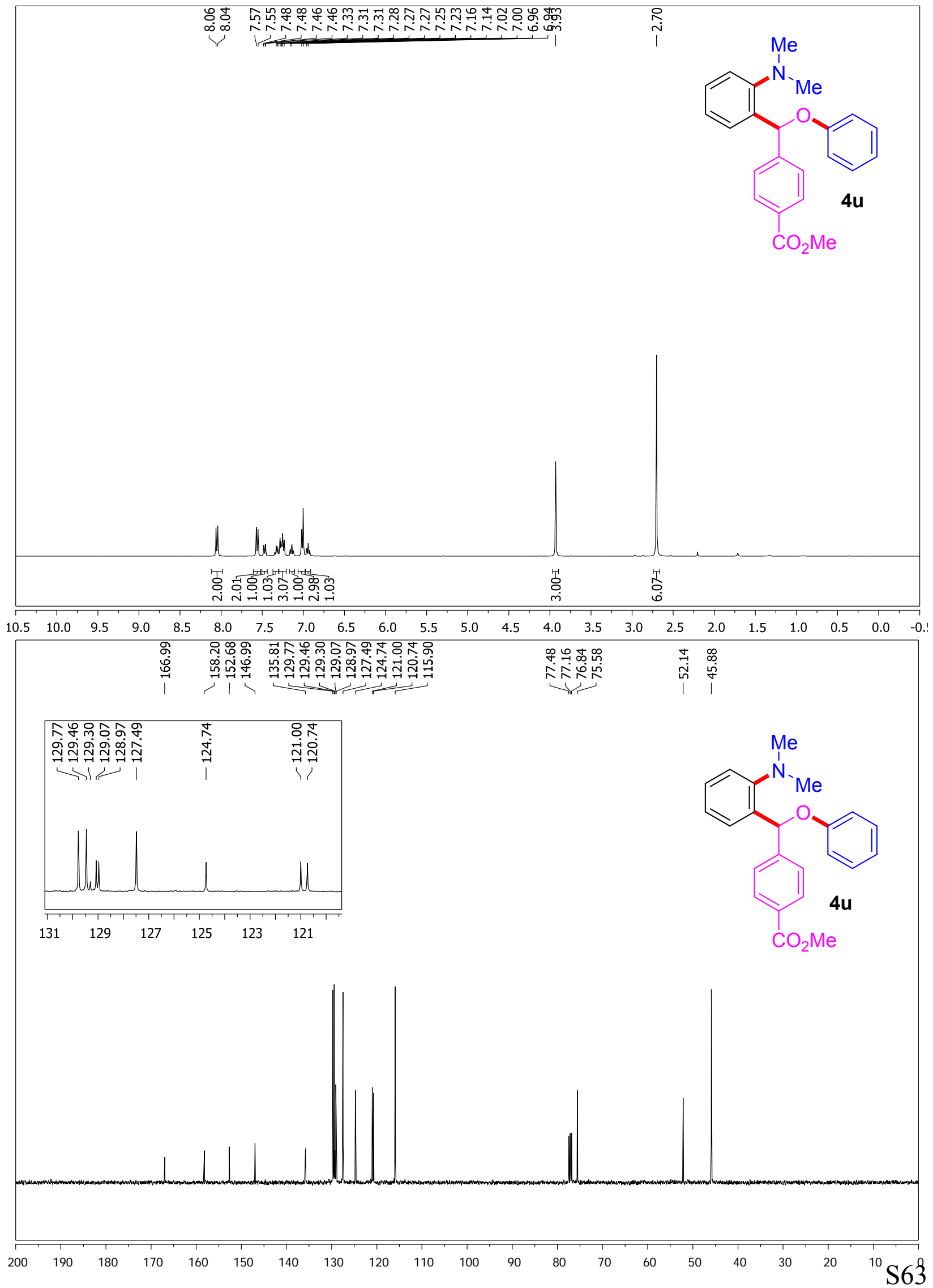




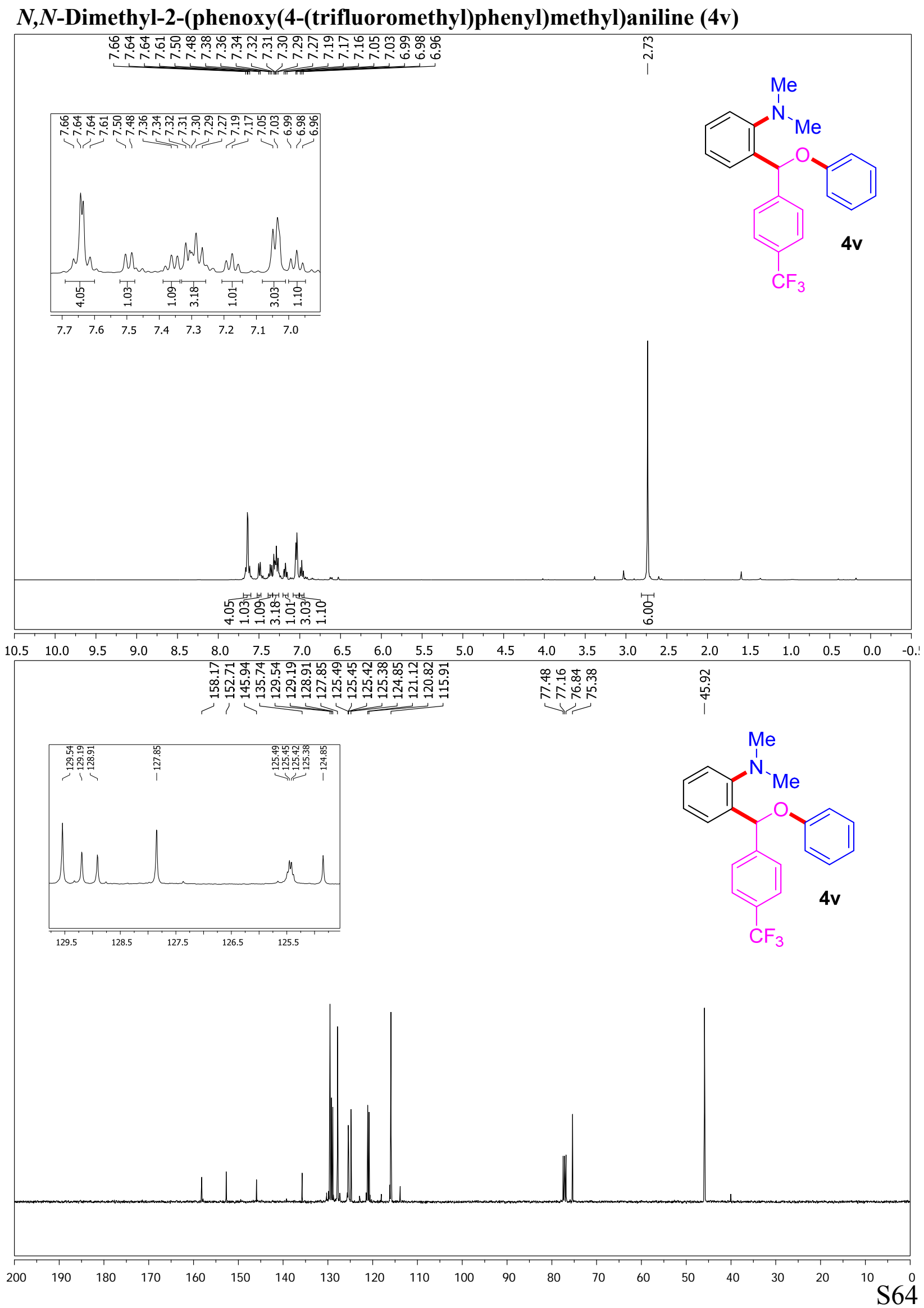


$N, N$-Dimethyl-2-((4-nitrophenyl)(phenoxy)methyl)aniline (4w)
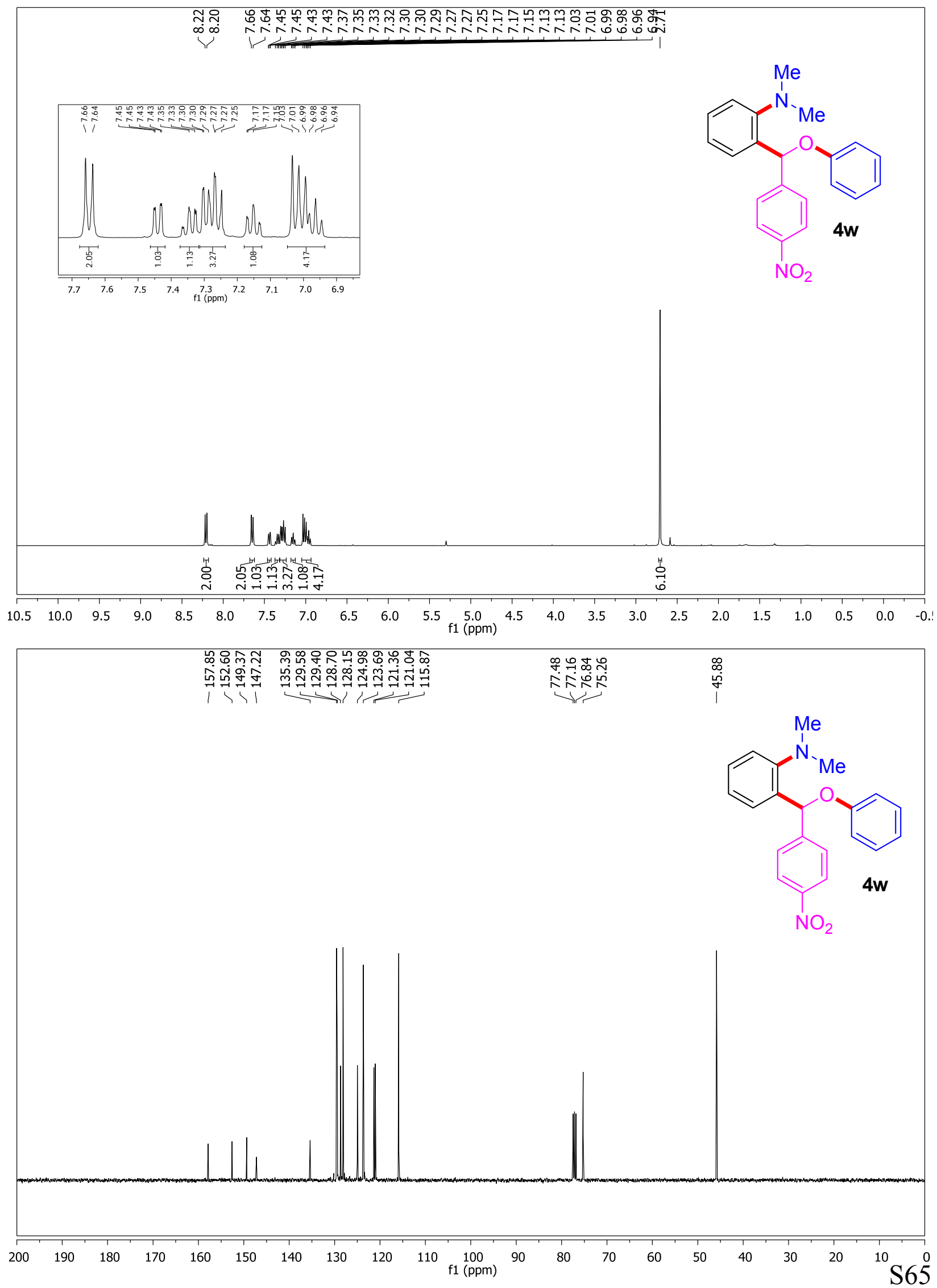


\section{2-((3-Bromophenyl)(phenoxy)methyl)- $N, N$-dimethylaniline (4x)}

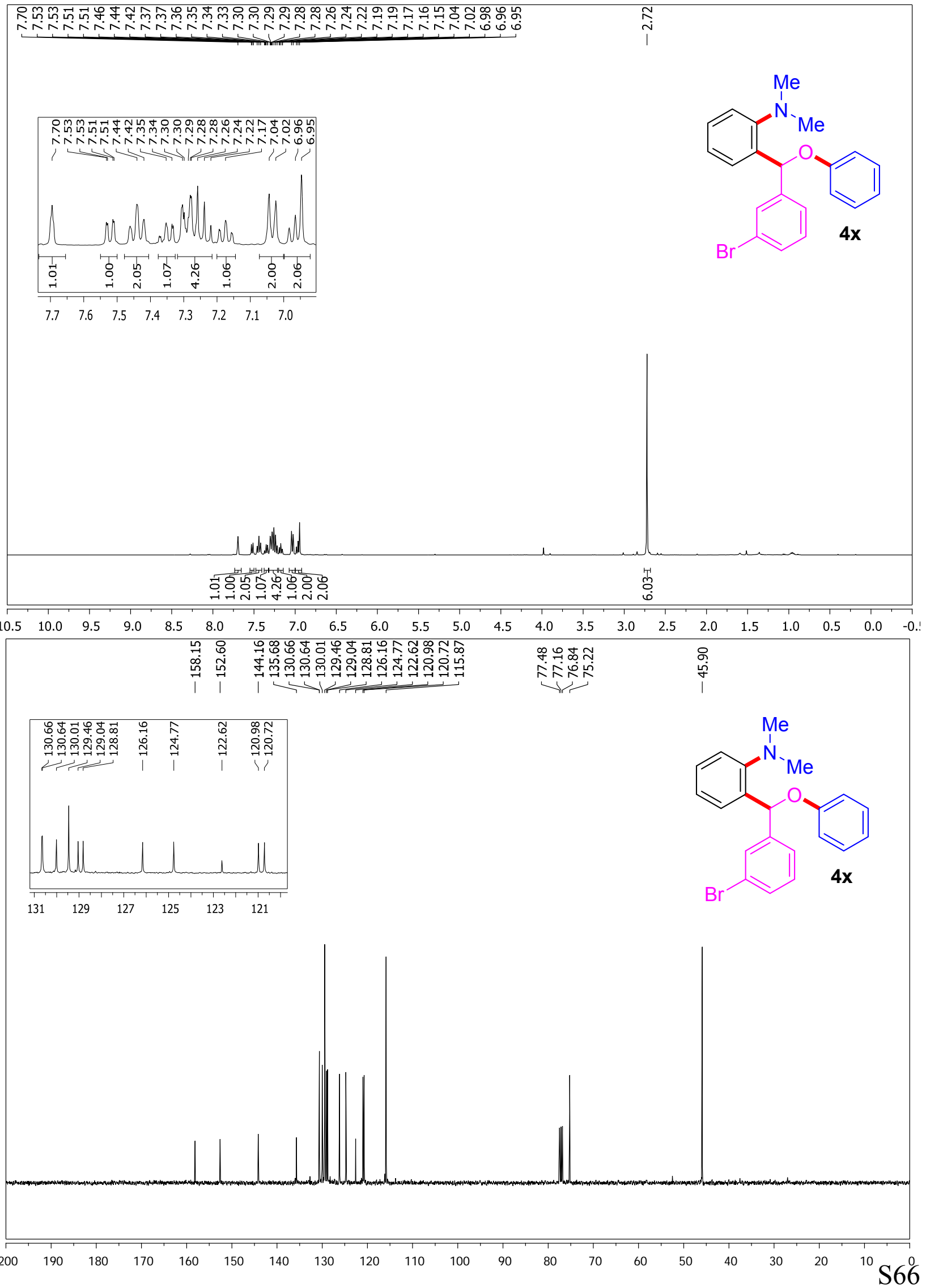


$N, N$-Dimethyl-2-((3-nitrophenyl)(phenoxy)methyl)aniline (4y)

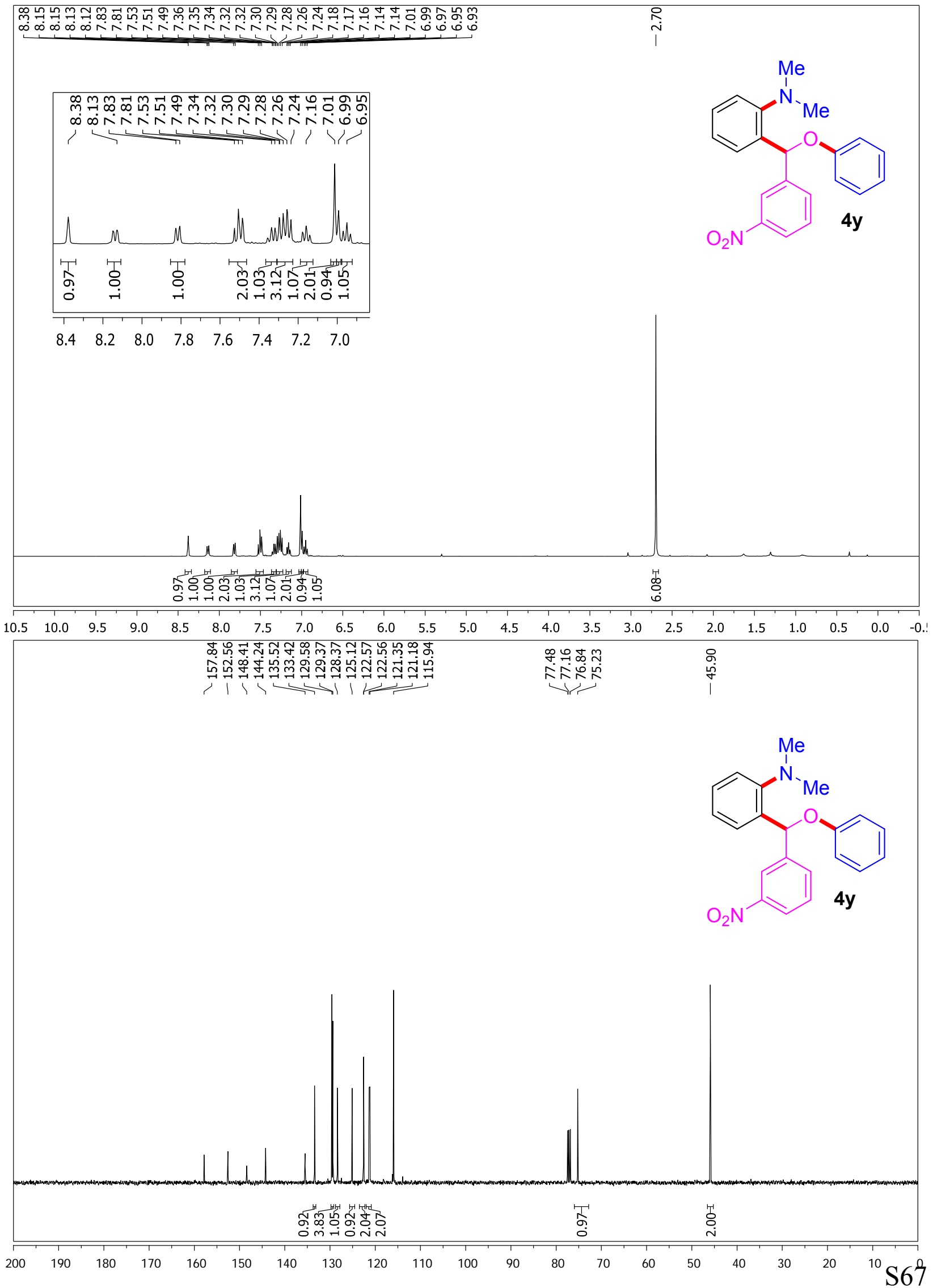




\section{2-((2-Fluorophenyl)(phenoxy)methyl)- $N, N$-dimethylaniline (4z)}

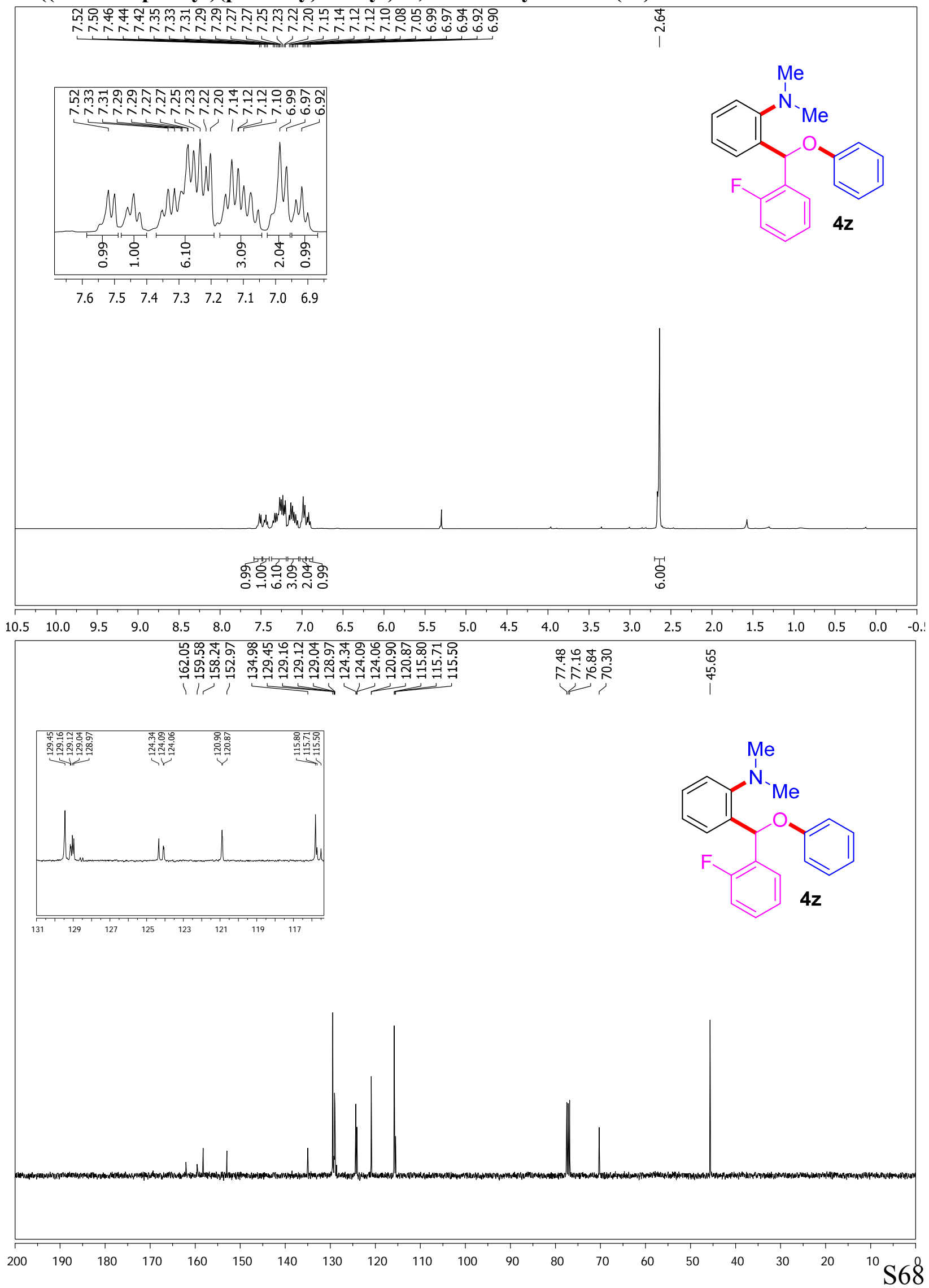




\section{$N, N$-Dimethyl-2-((2-nitrophenyl)(phenoxy)methyl)aniline (4aa)}
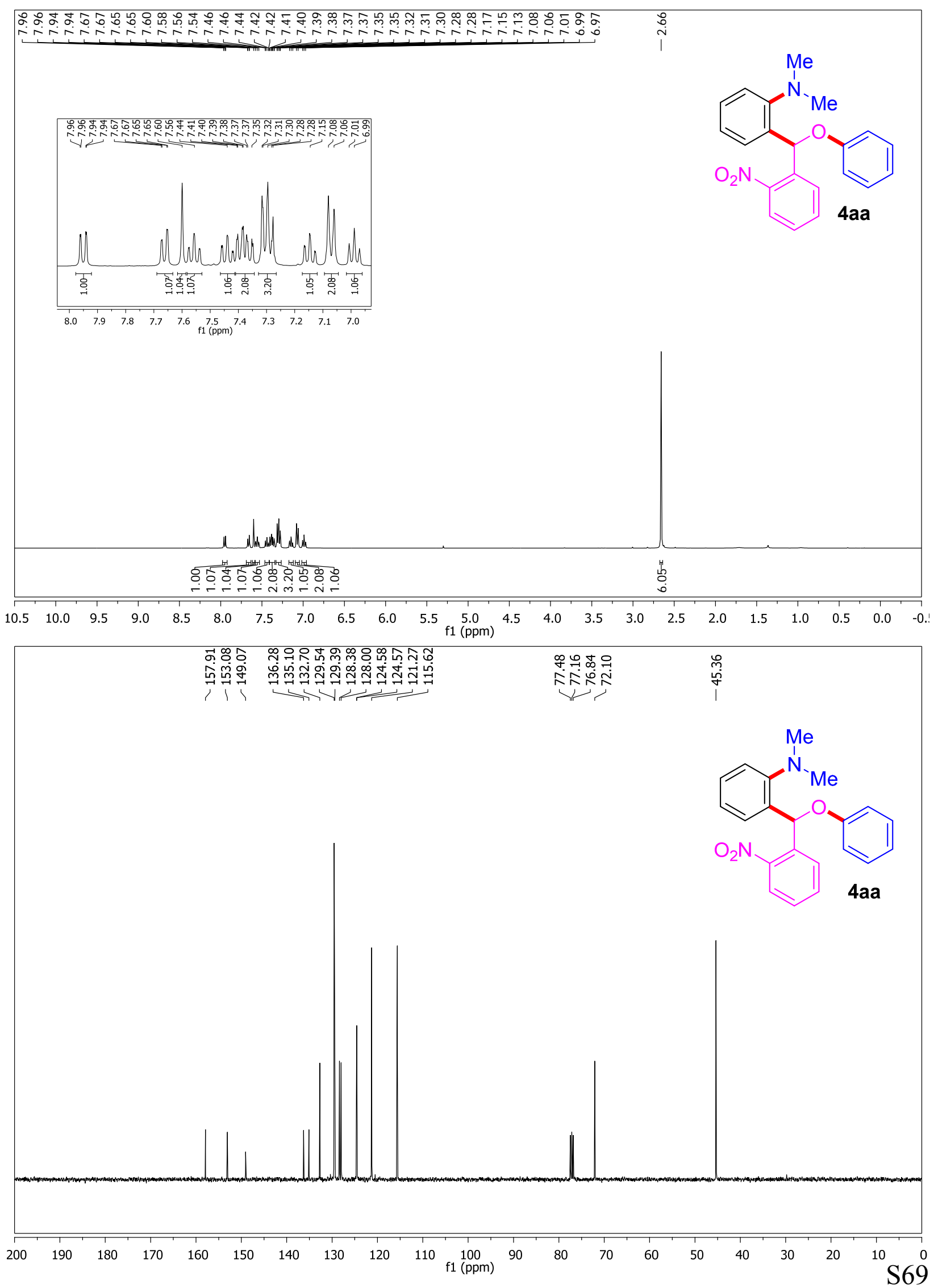


\section{2-((3,4-Dichlorophenyl)(phenoxy)methyl)- $N, N$-dimethylaniline (4ab)}
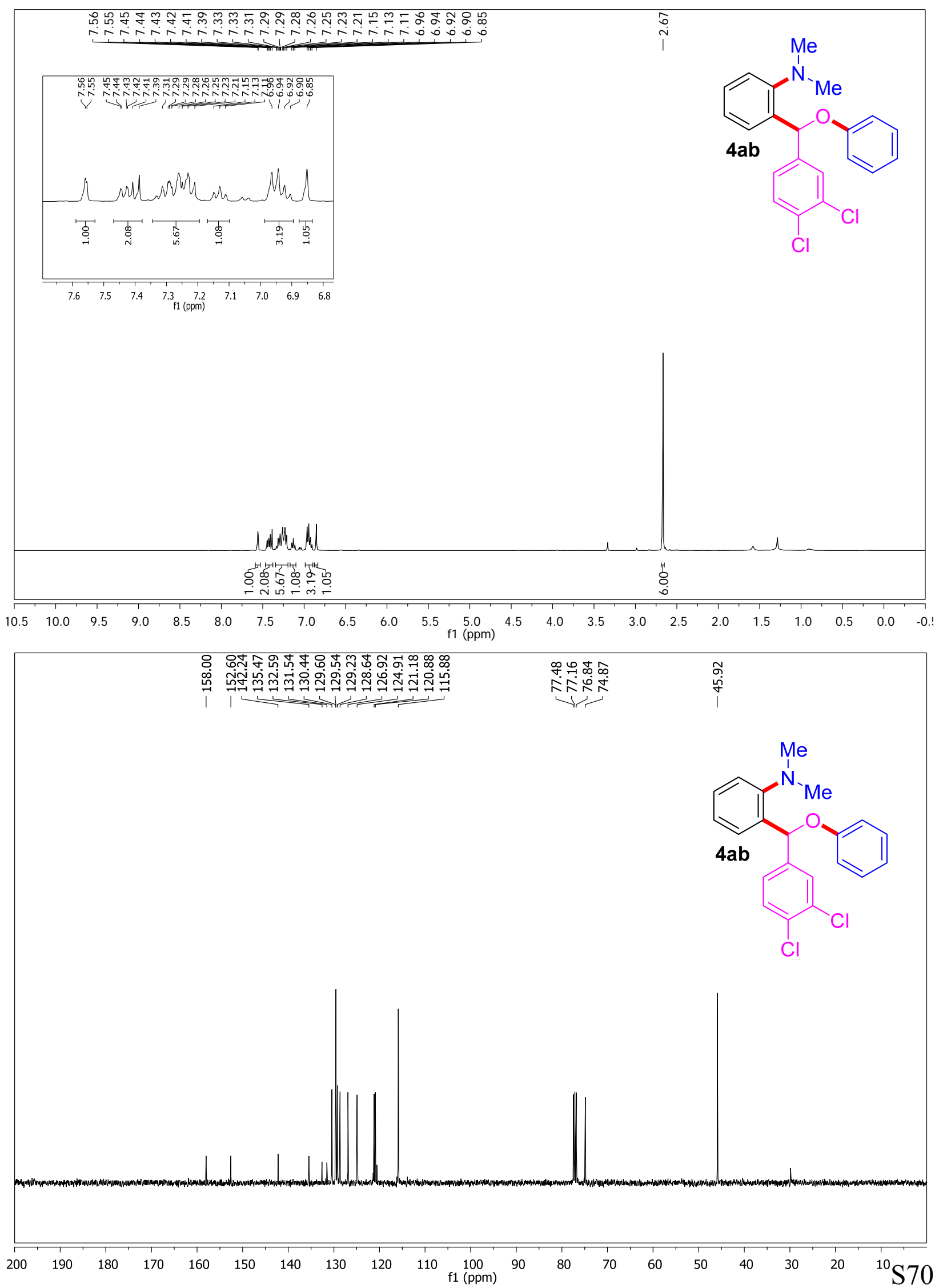


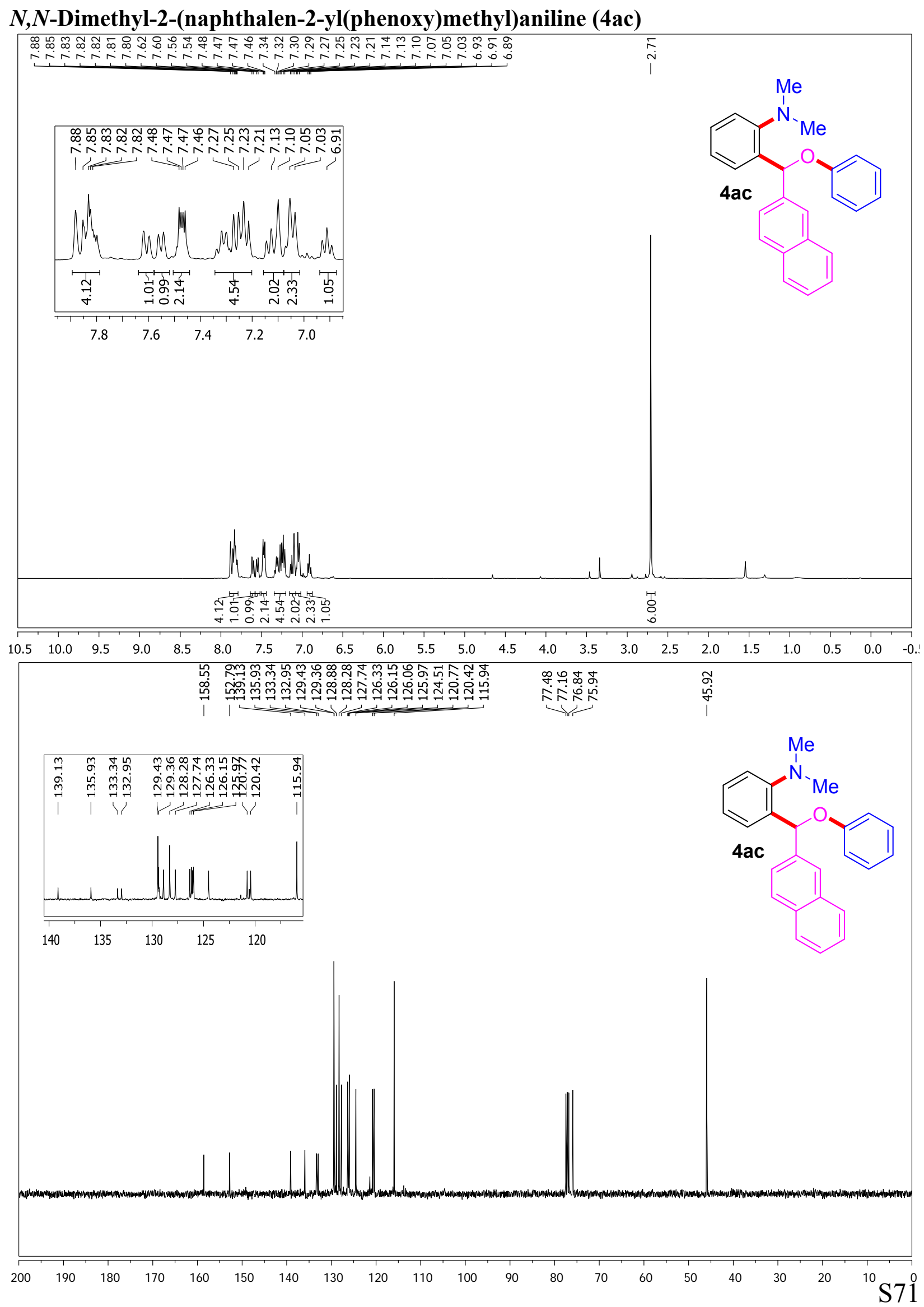


$N, N$-Dimethyl-2-(phenoxy(thiophen-2-yl)methyl)aniline (4ad)

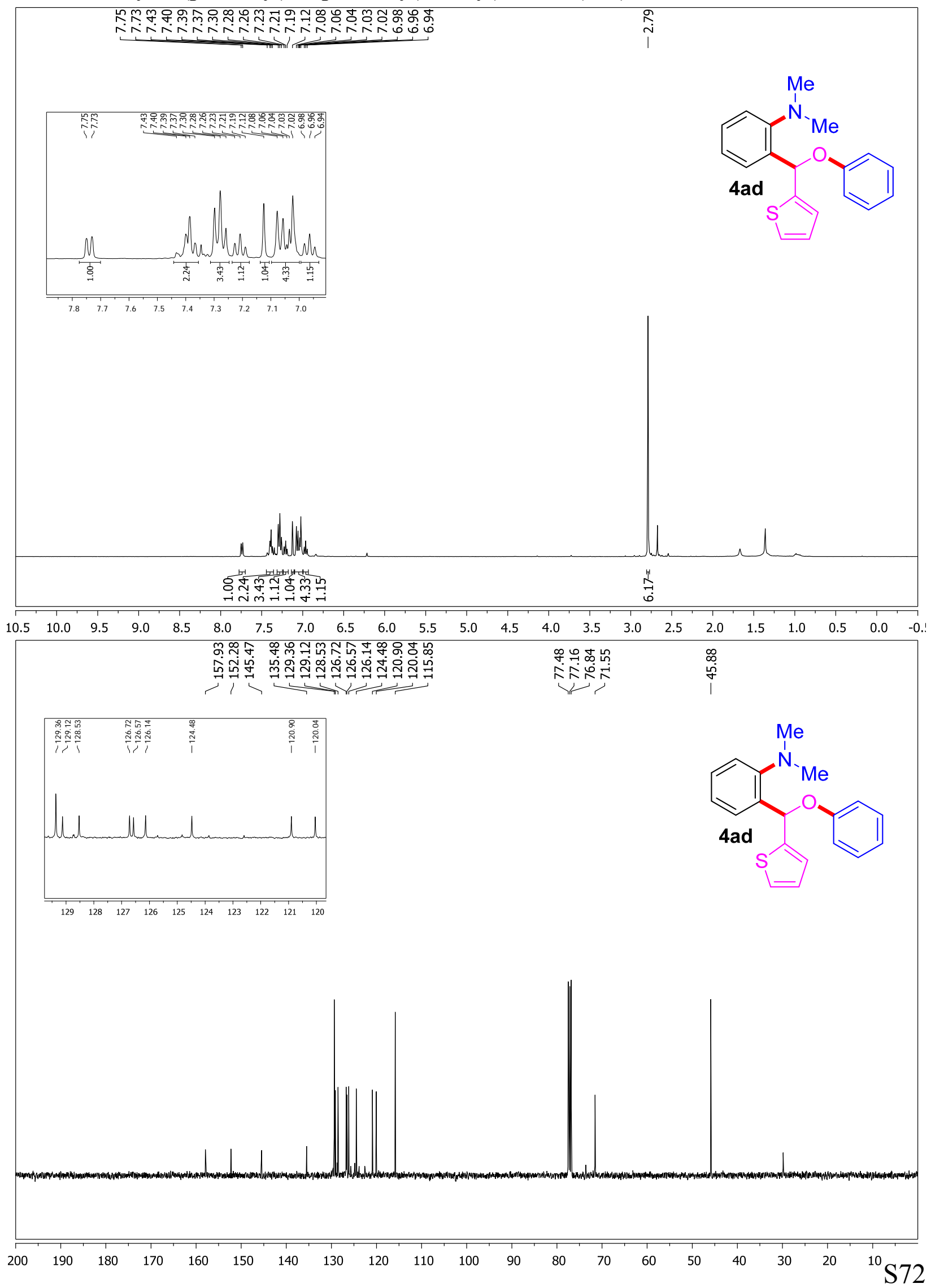




\section{$N, N$-Dimethyl-2-(1-phenoxydecyl)aniline (4ae)}

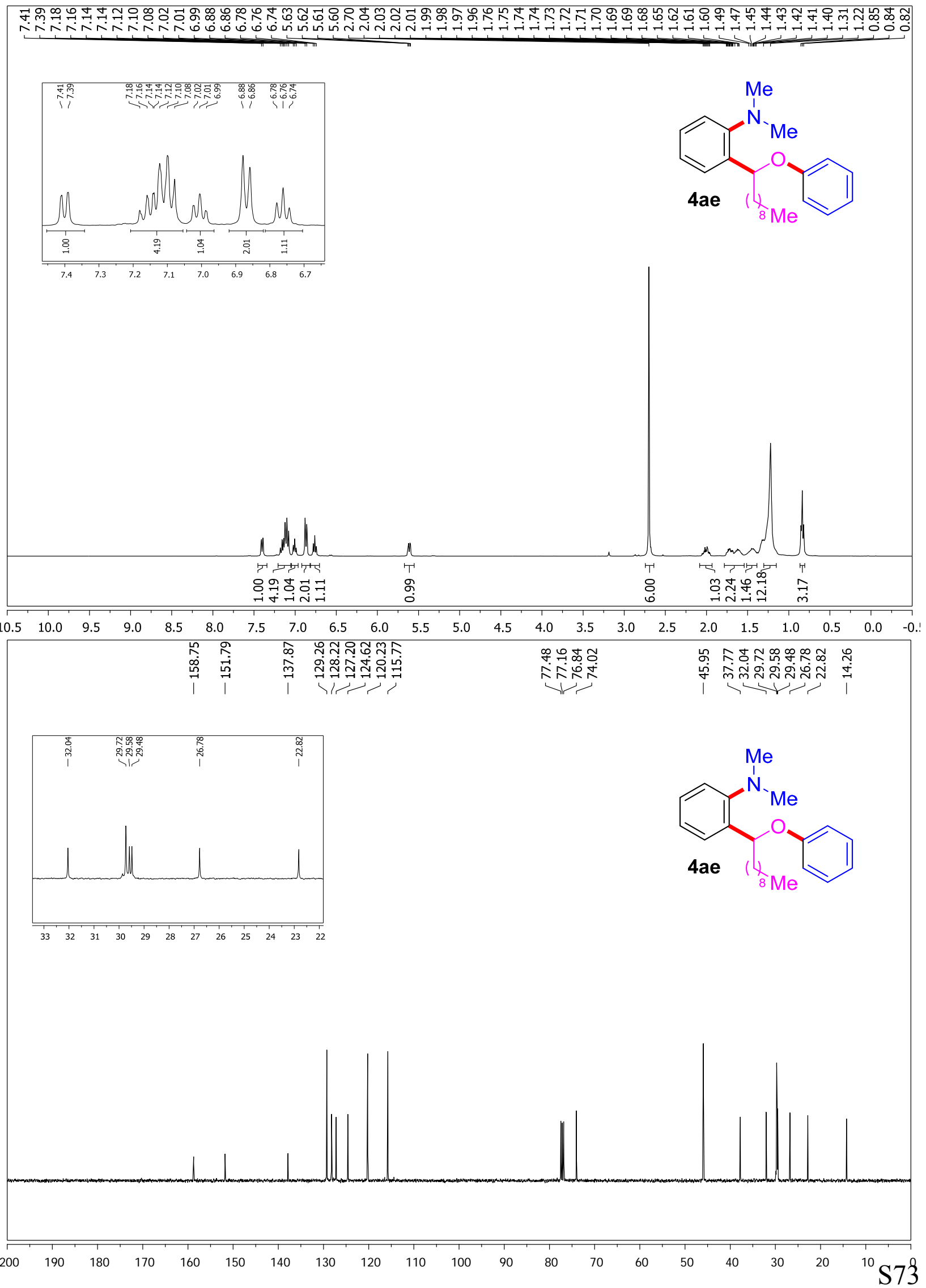




\section{2-(Cyclohexyl(phenoxy)methyl)- $N, N$-dimethylaniline (4af)}

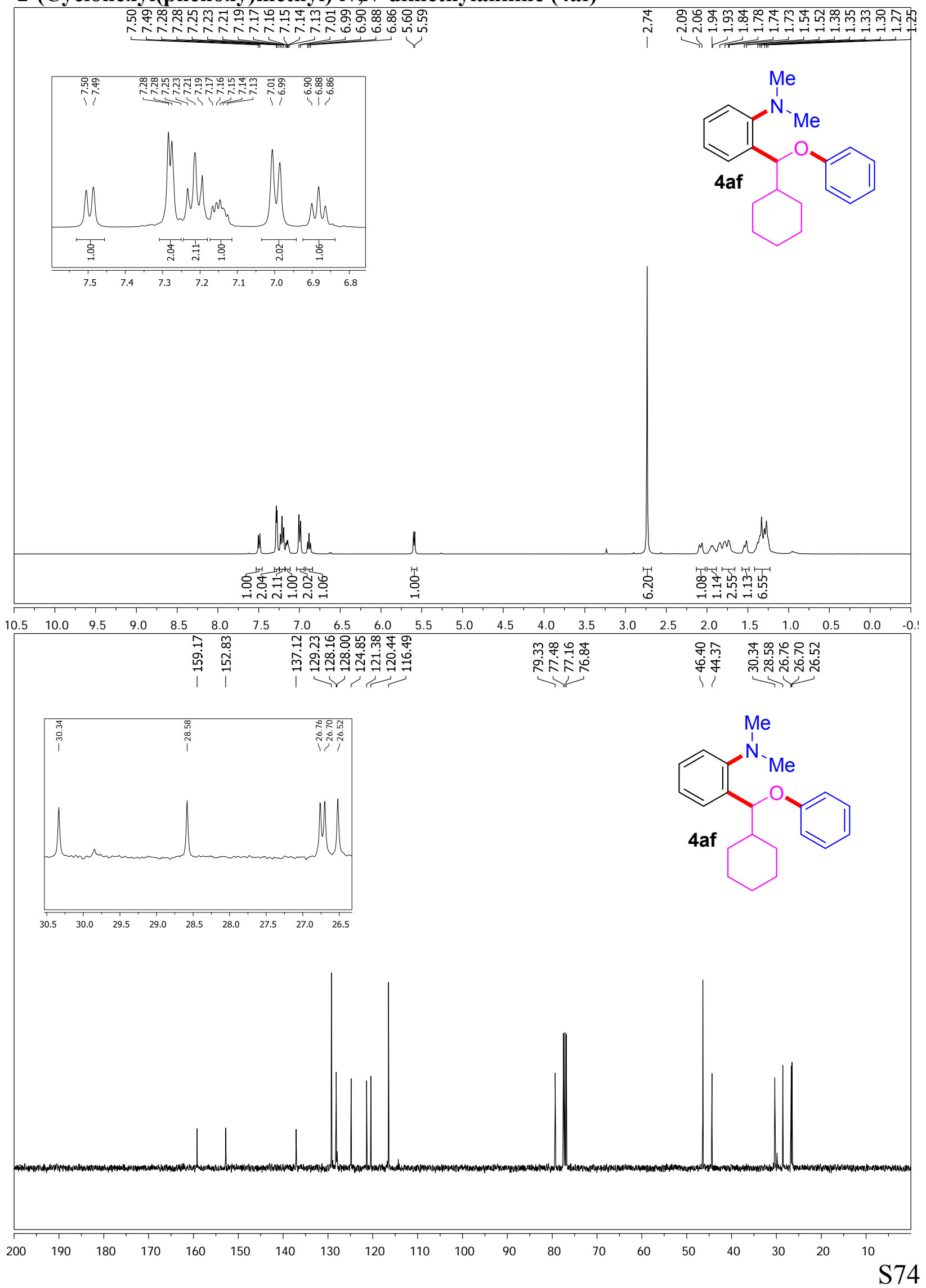




\section{4-((2-(Dimethylamino)-4,5-dimethylphenyl)(phenoxy)methyl)benzonitrile (4ag)}

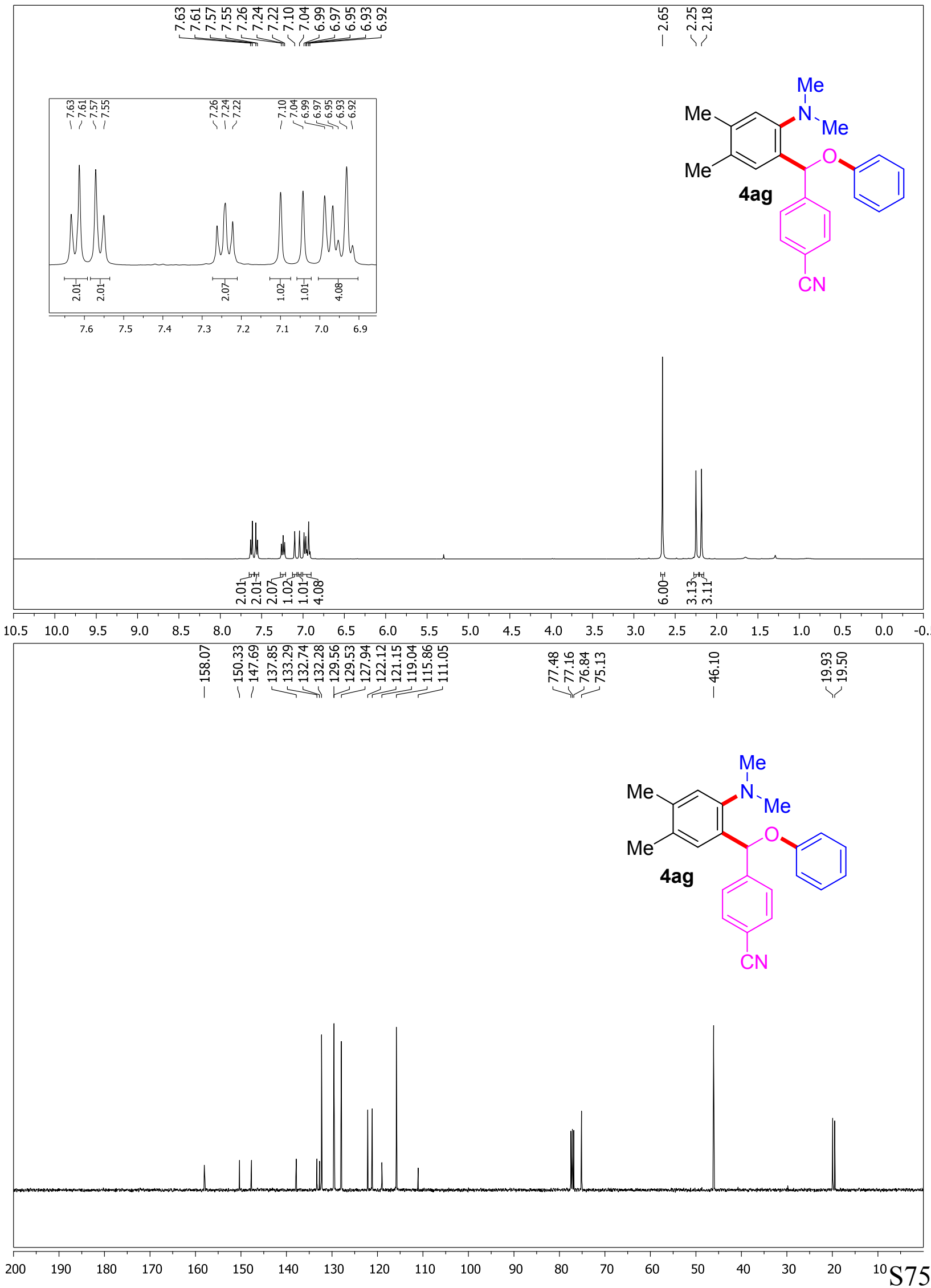



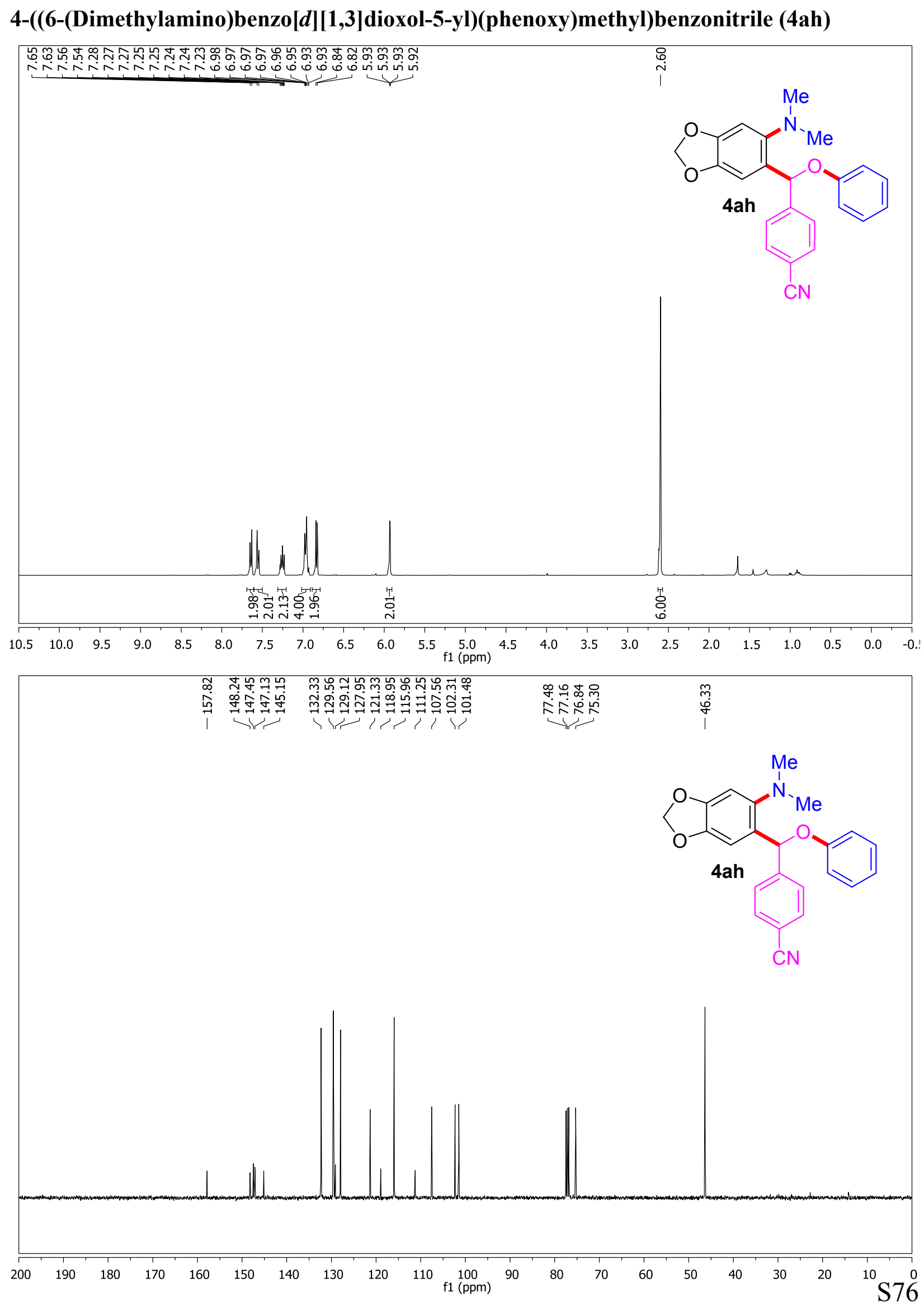


\section{4-((2-(Dimethylamino)-4,5-difluorophenyl)(phenoxy)methyl)benzonitrile (4ai)}
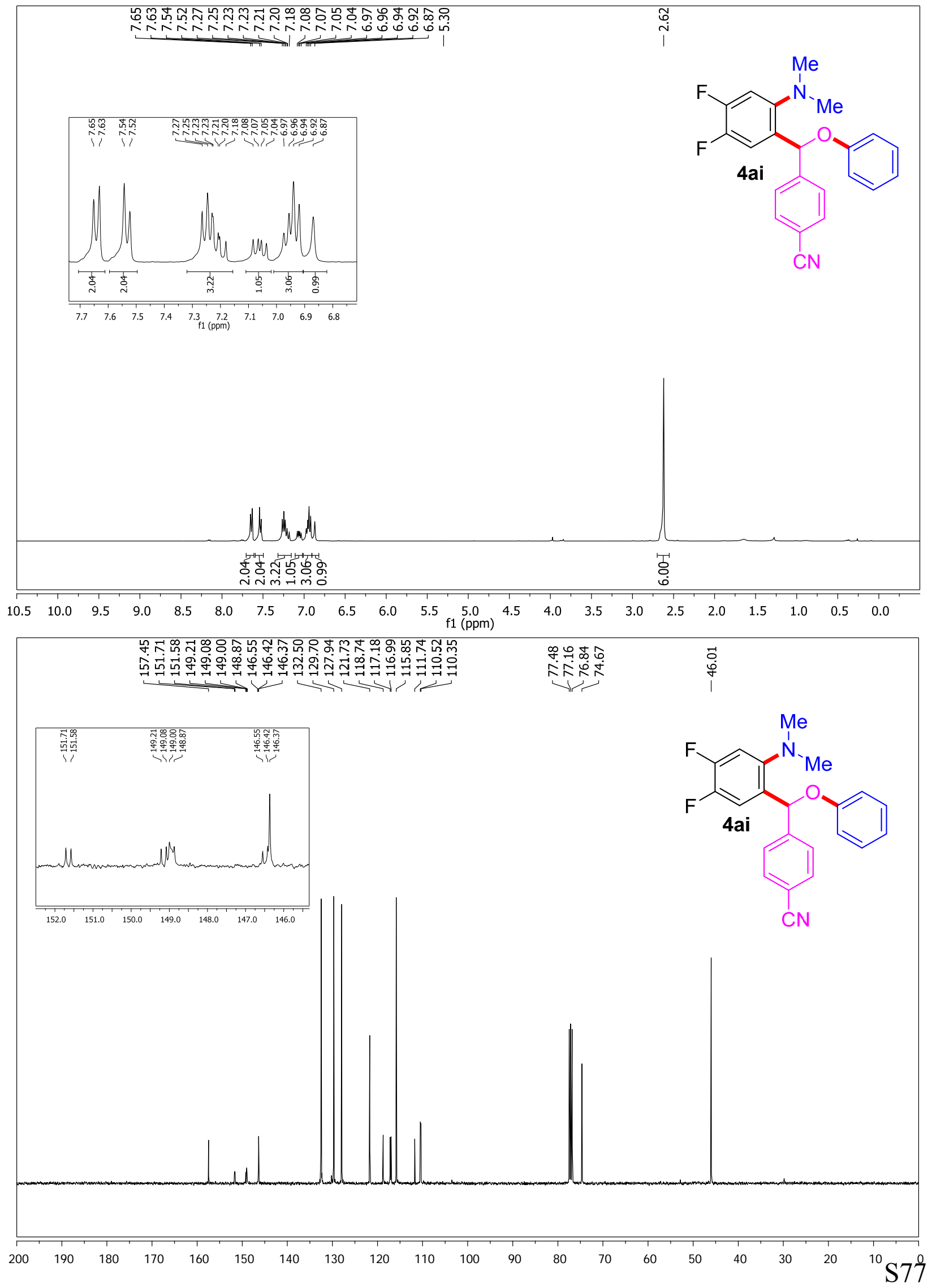
4-((3-(Dimethylamino)naphthalen-2-yl)(phenoxy)methyl)benzonitrile (4aj)

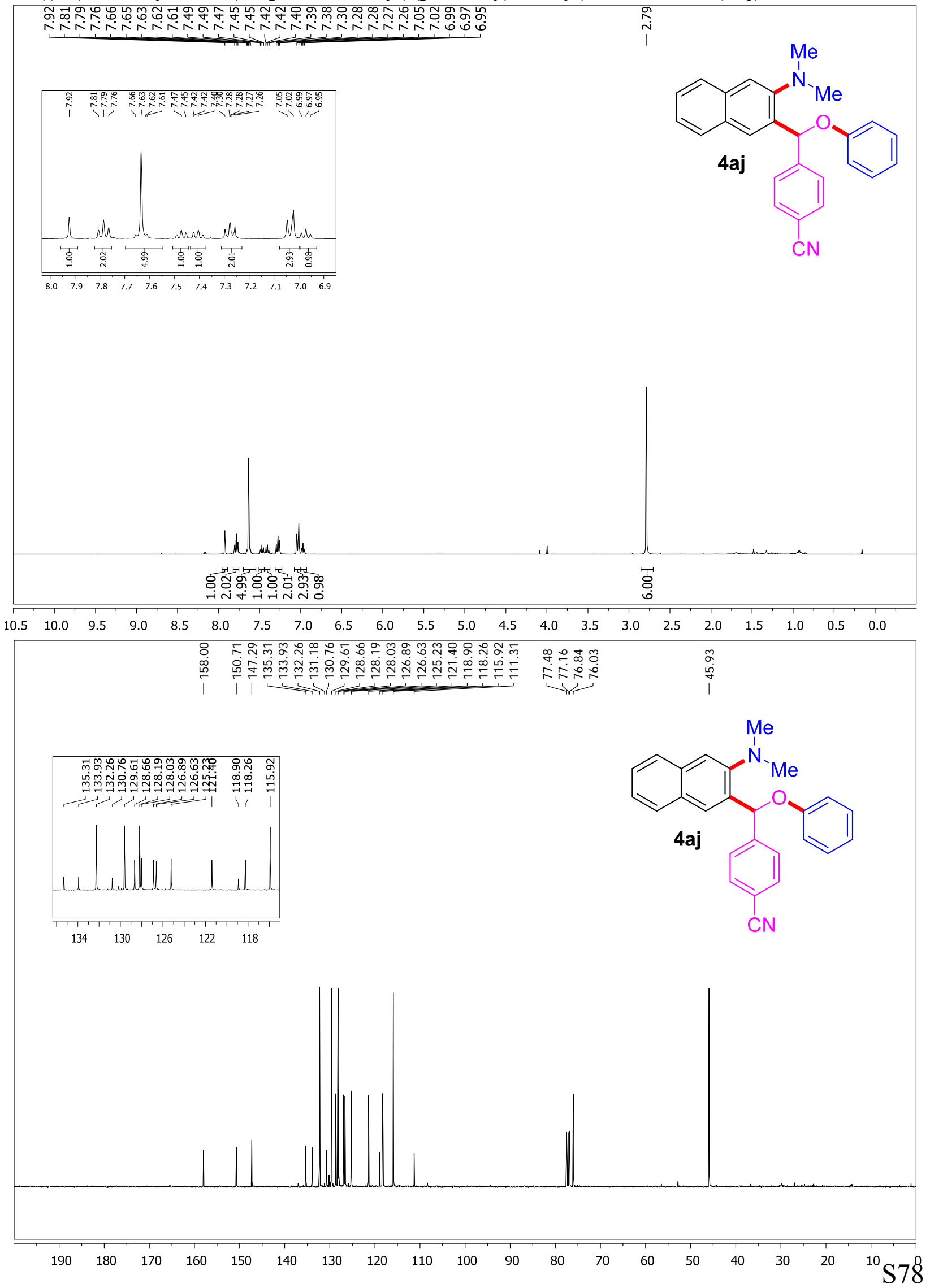




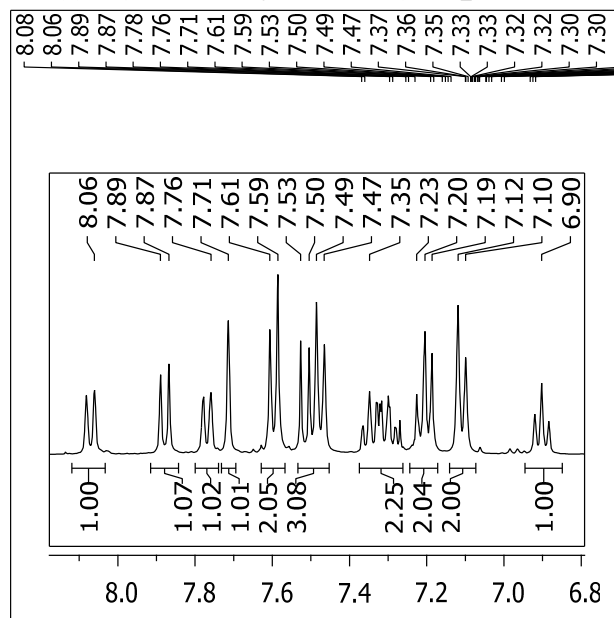

$\underset{i}{\infty}$
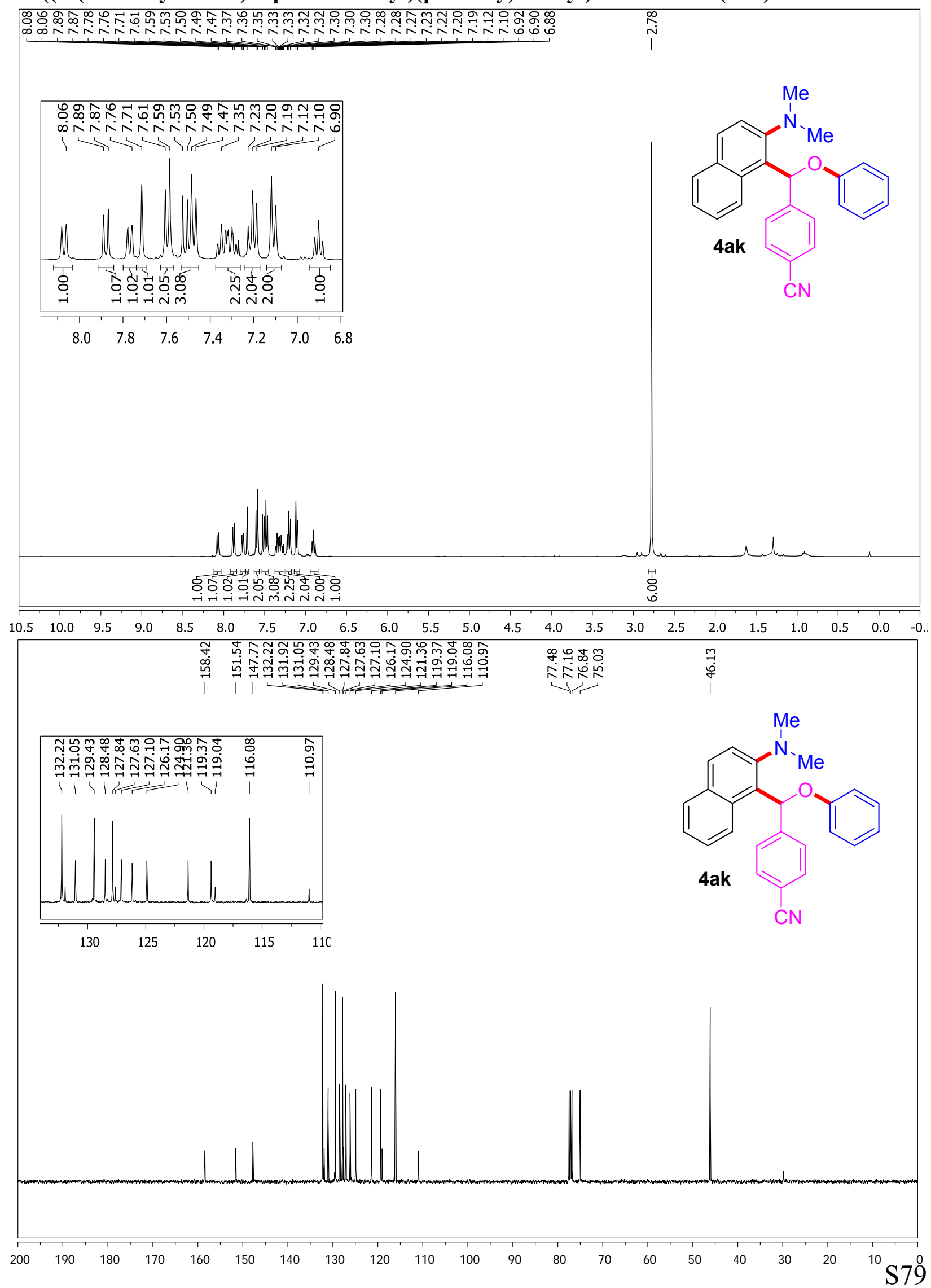
4-((2-(Dimethylamino)-5-methylphenyl)(phenoxy)methyl)benzonitrile (4al) and 4-((2-(Dimethylamino)-4-methylphenyl)(phenoxy)methyl)benzonitrile (4al')
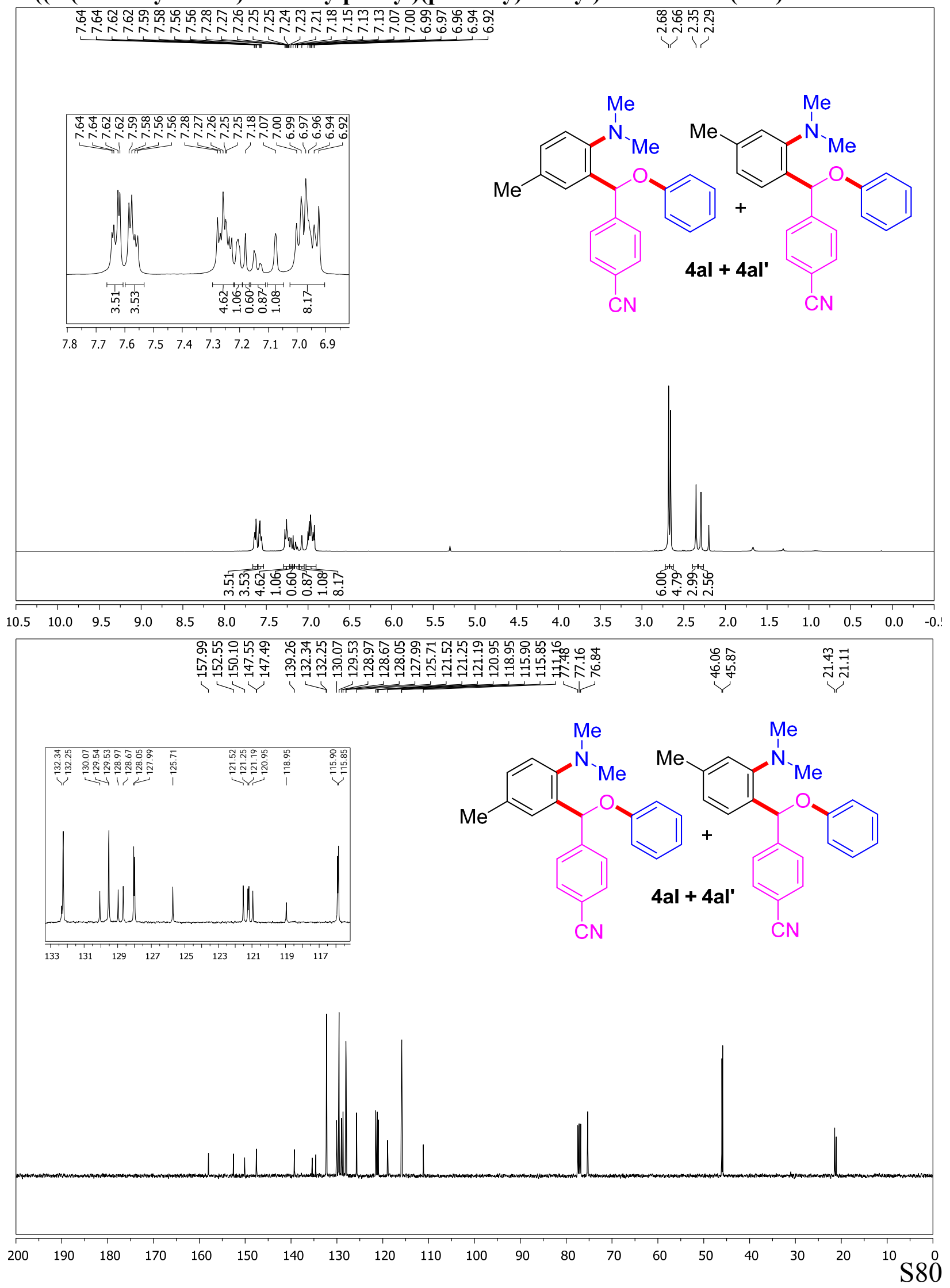
4-((2-(Dimethylamino)-5-fluorophenyl)(phenoxy)methyl)benzonitrile (4am) and 4-((2-(Dimethylamino)-4-fluorophenyl)(phenoxy)methyl)benzonitrile (4am')

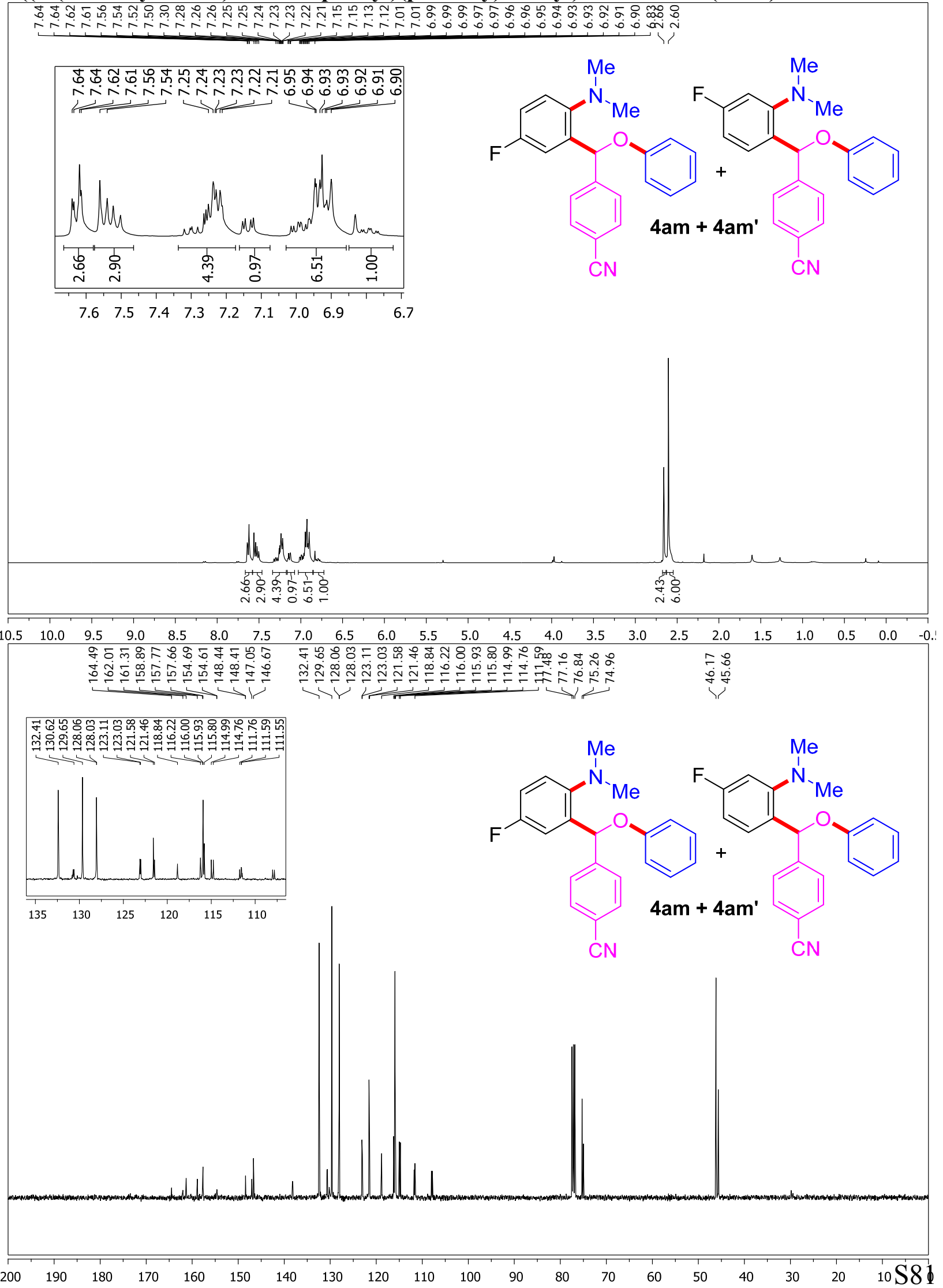


3-(2-(Dimethylamino)phenyl)-1-methyl-3-phenoxyindolin-2-one (6a)

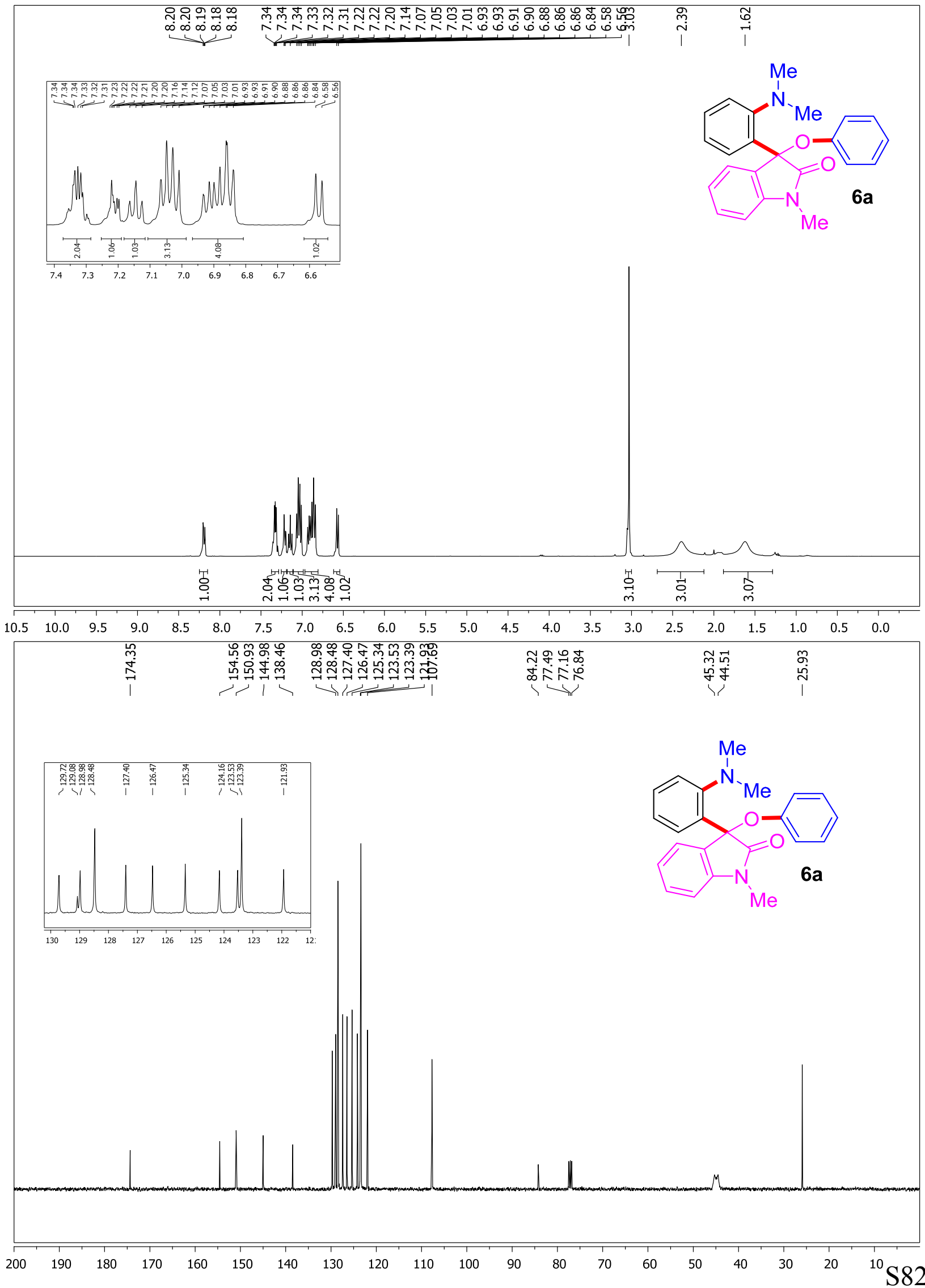




\section{5-Bromo-3-(2-(dimethylamino)phenyl)-1-methyl-3-phenoxyindolin-2-one (6b)}

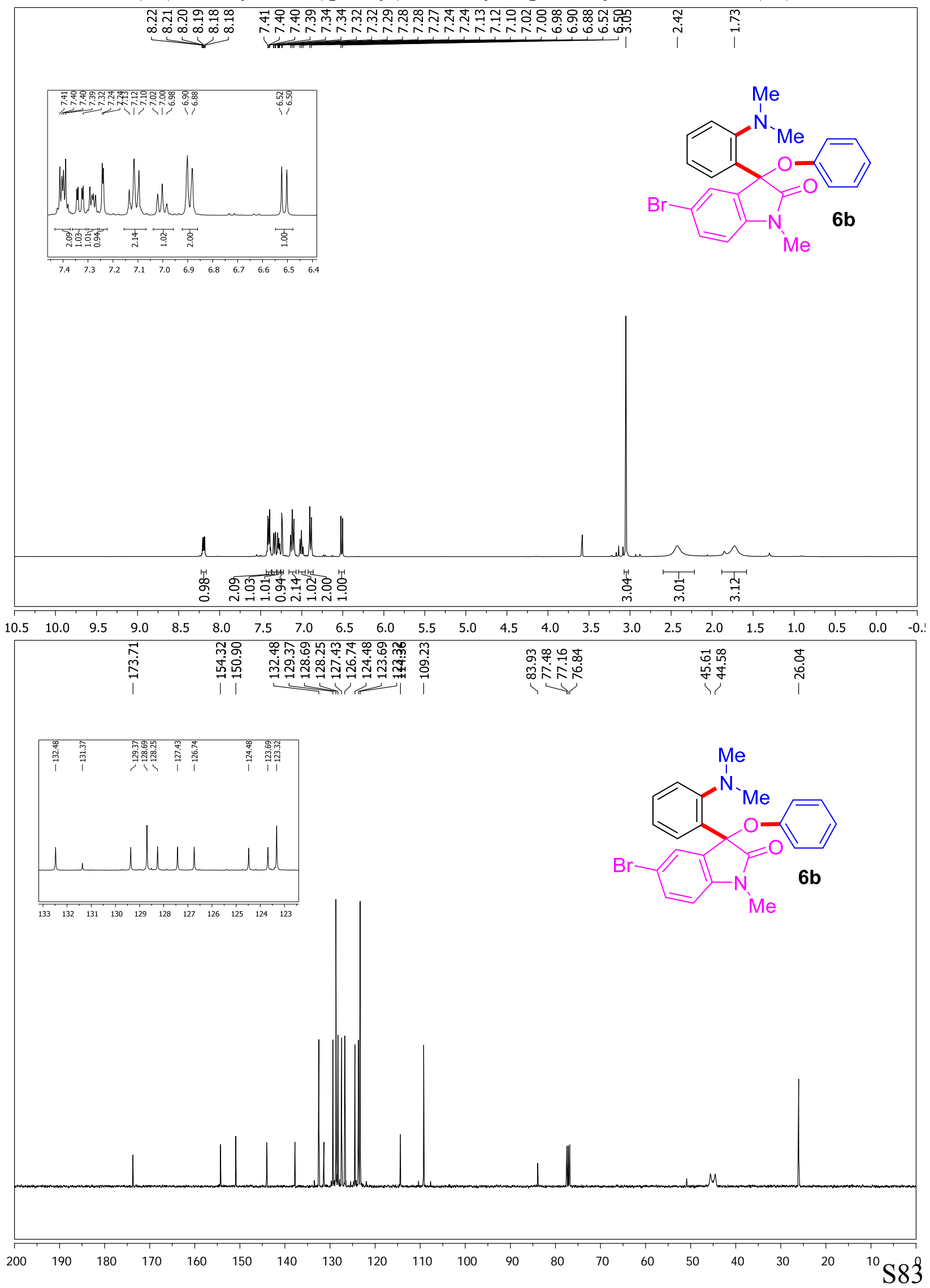


3-(2-(Dimethylamino)phenyl)-5-fluoro-1-methyl-3-phenoxyindolin-2-one (6c)

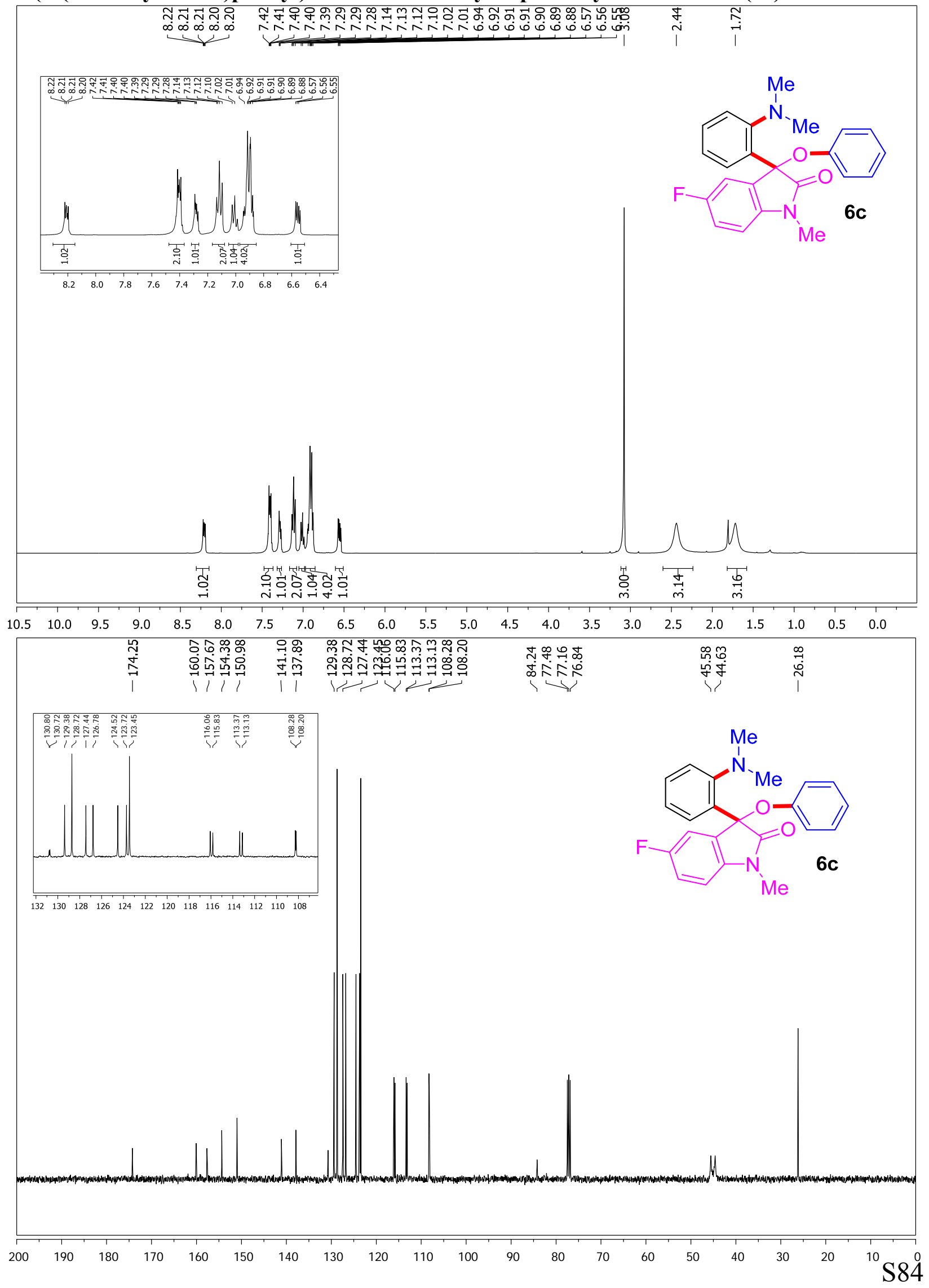




\section{3-(2-(Dimethylamino)phenyl)-1-methyl-3-(p-tolyloxy)indolin-2-one (6d)}
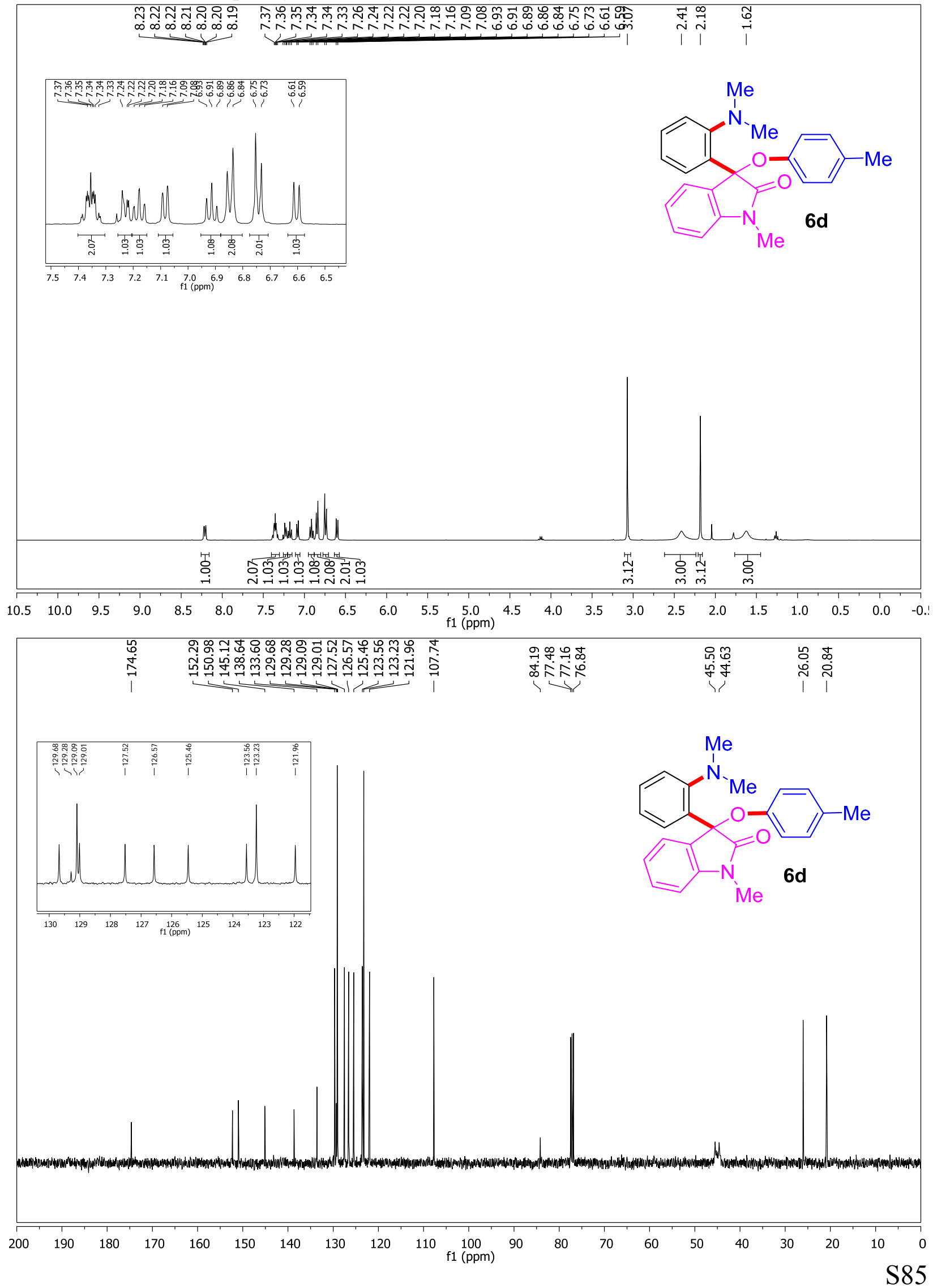
1-Benzyl-3-(2-(dimethylamino)phenyl)-3-phenoxyindolin-2-one (6e)

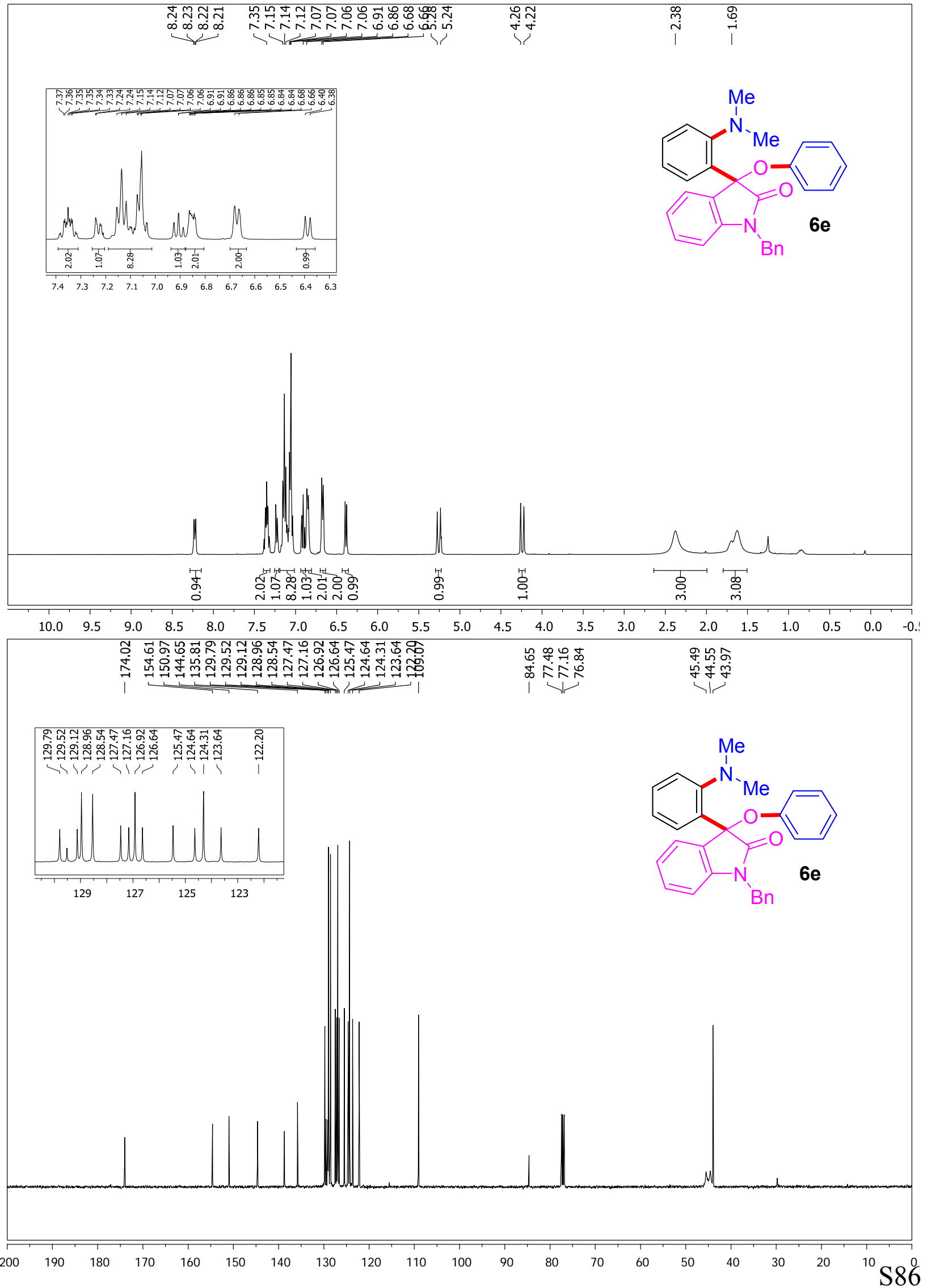


$N, N$-Dimethyl-2-(2,2,2-trifluoro-1-phenoxy-1-phenylethyl)aniline (8)

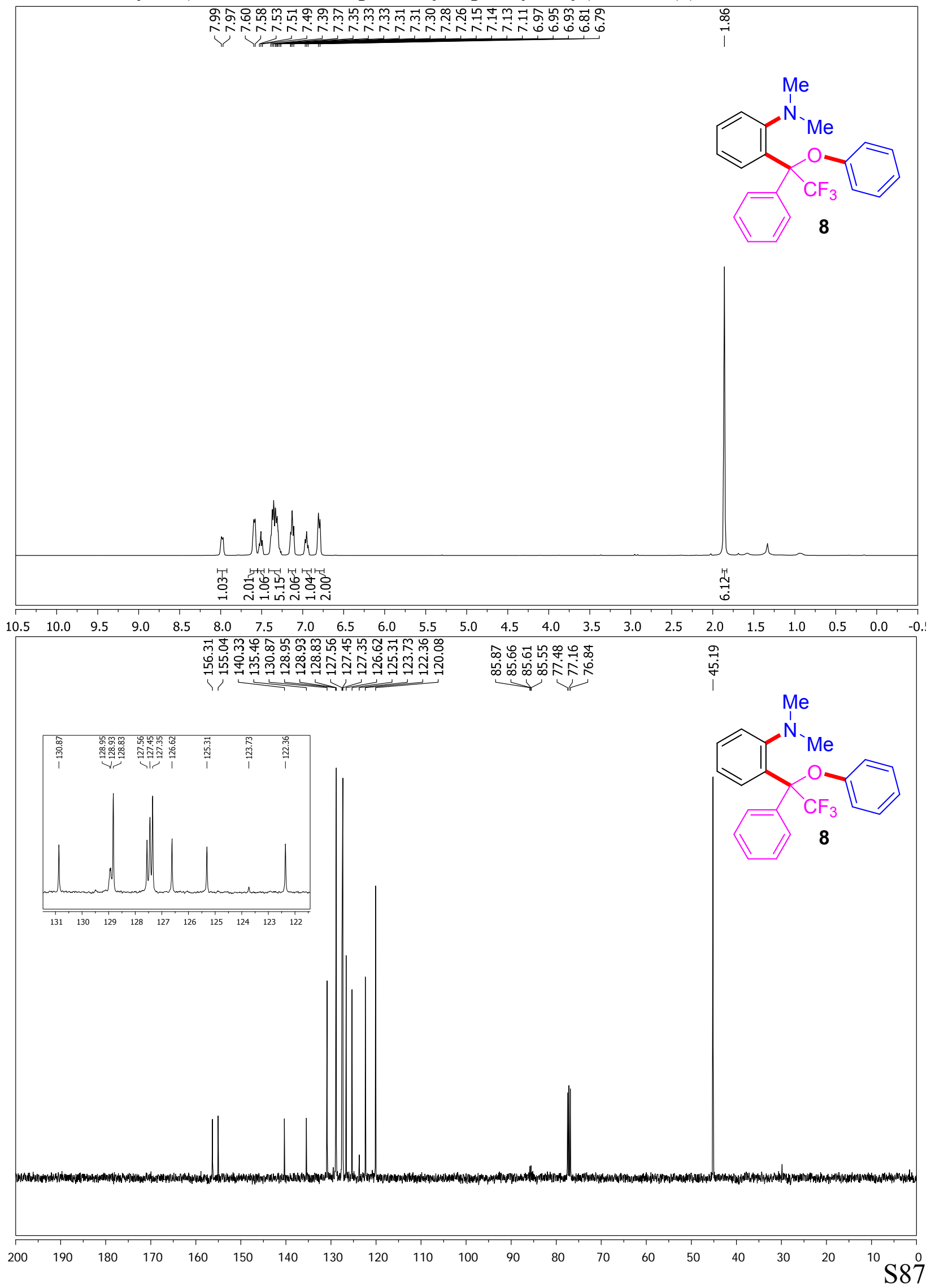


2-(2-(Dimethylamino)phenyl)-2-phenoxy-1,2-diphenylethan-1-one (10)

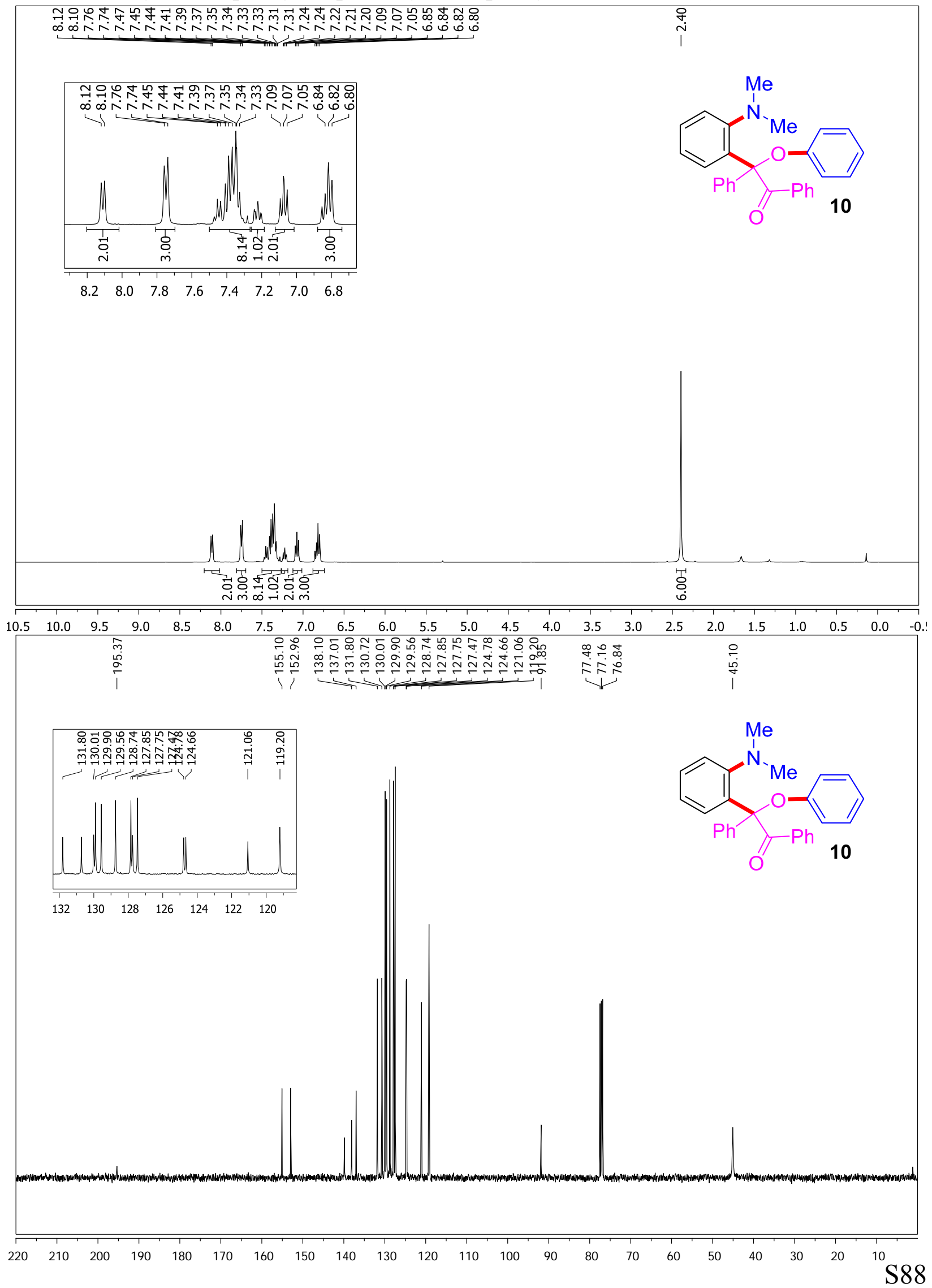




\section{4-(Hydroxy(2-(methyl(phenyl)amino)phenyl)methyl)benzonitrile (16)}

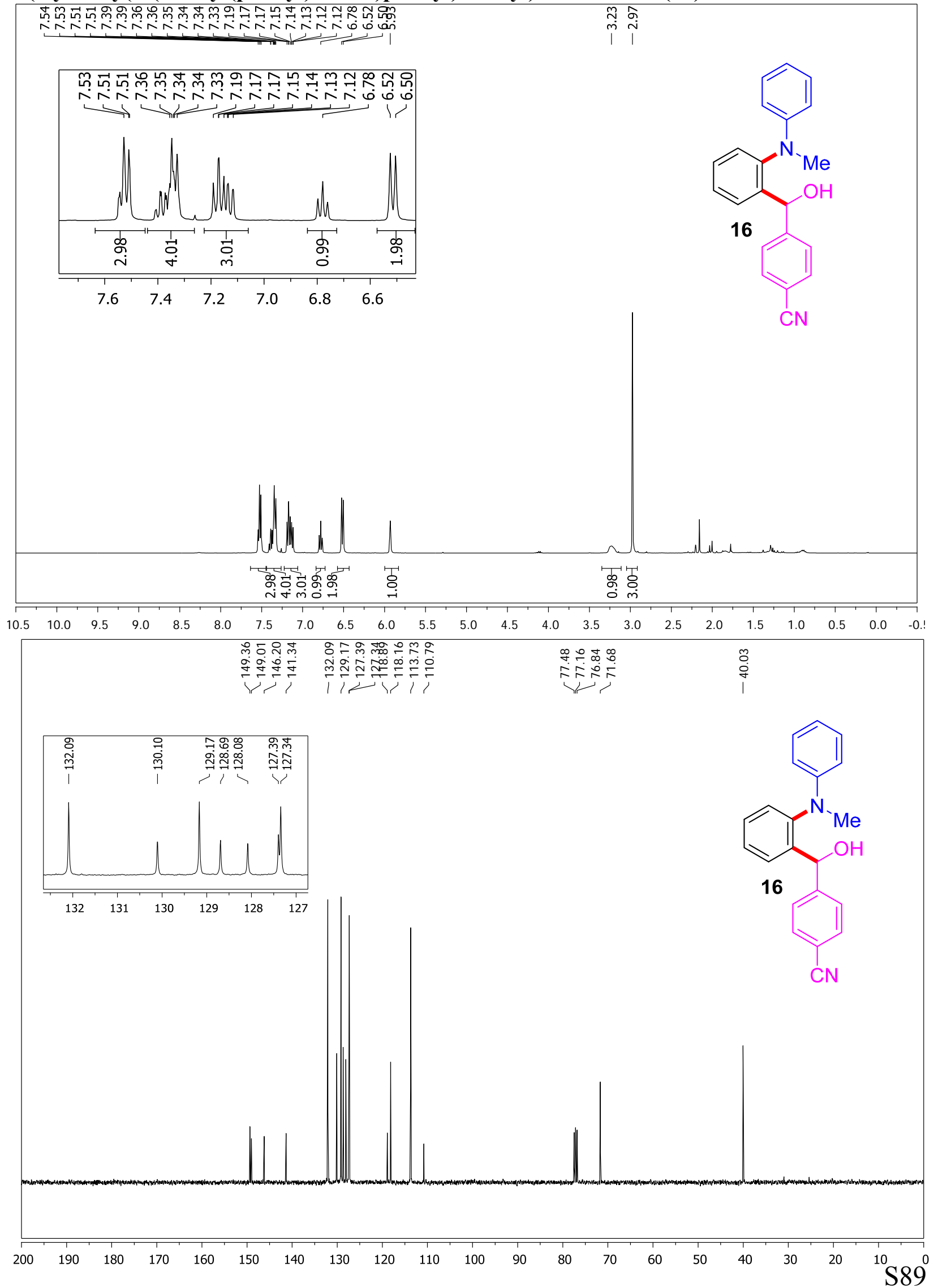




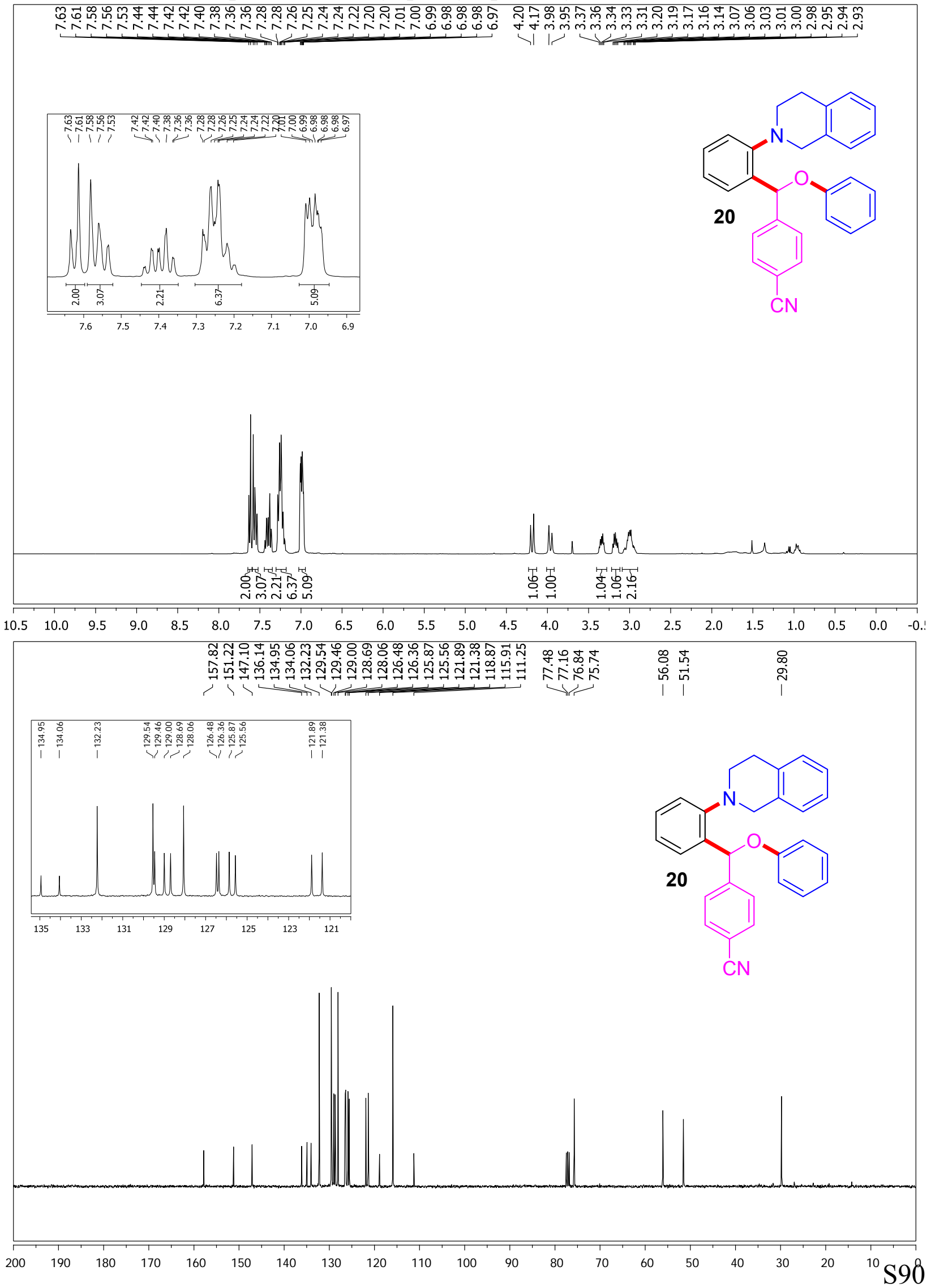


4-(Hydroxy(2-(phenyl(2-vinylbenzyl)amino)phenyl)methyl)benzonitrile (21)
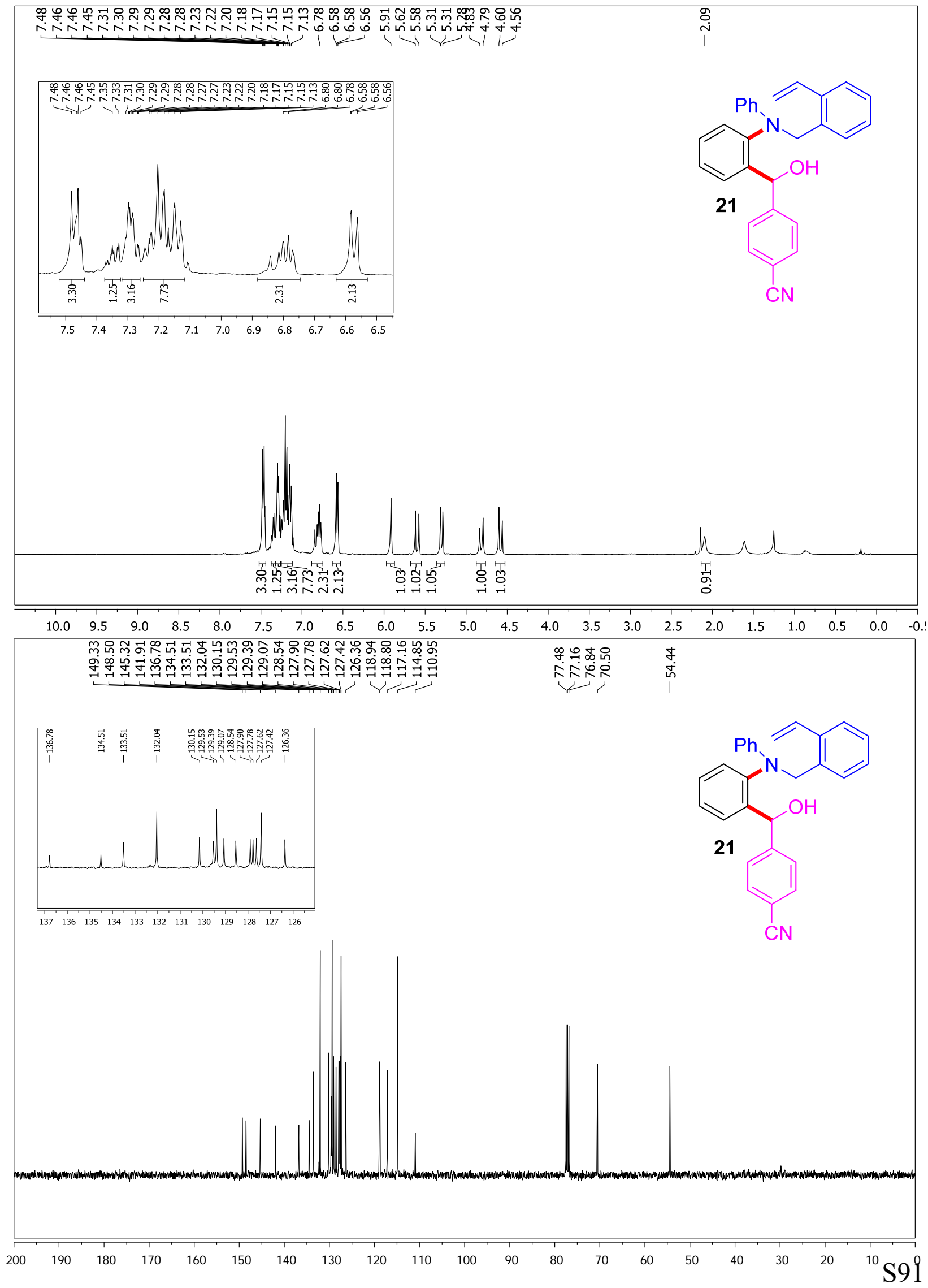
4-((2-((2(Dimethylamino)phenyl)(4-nitrophenyl)methoxy)phenyl)(phenoxy)methyl)benzoni trile (22)
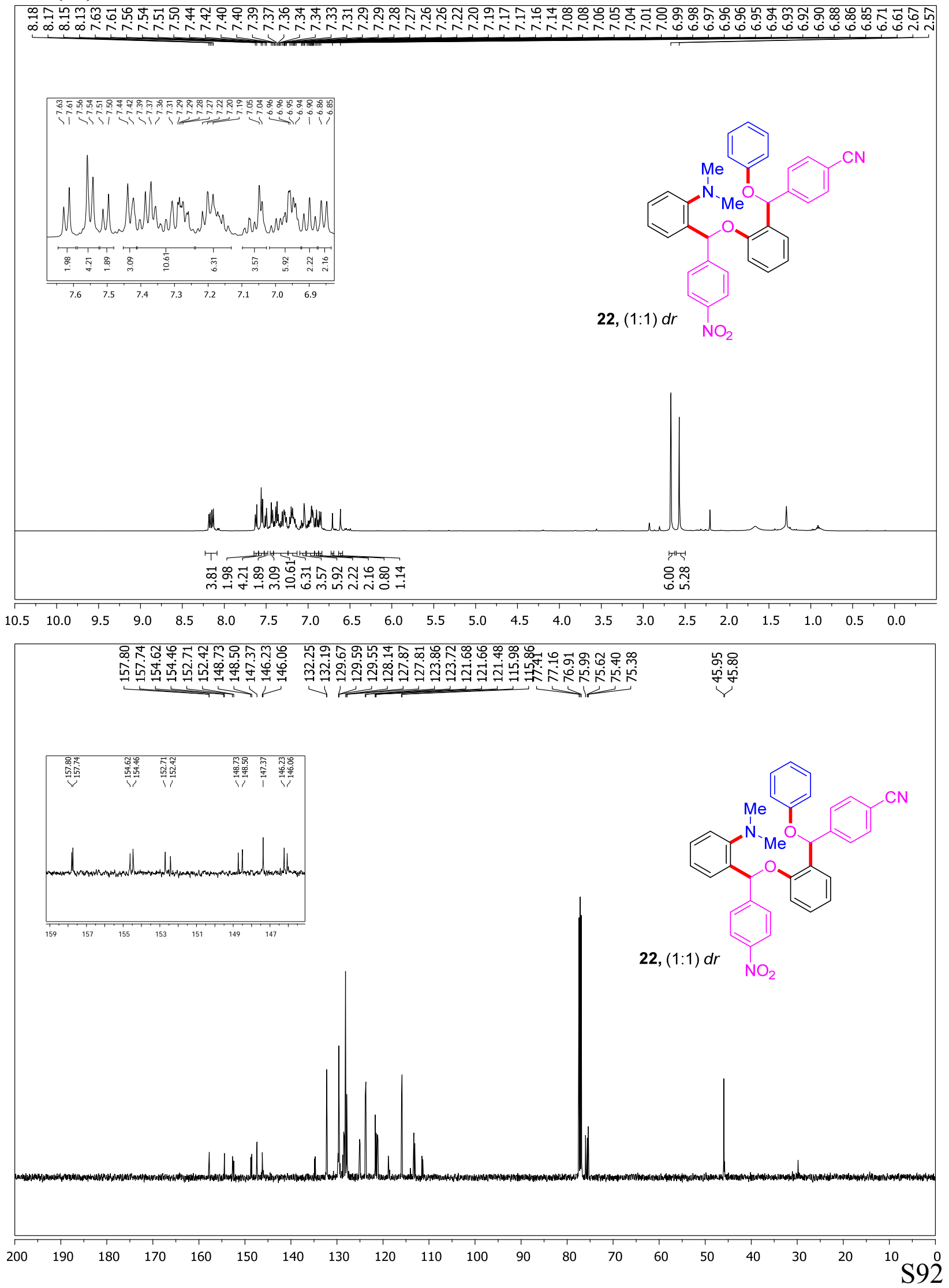